\title{
Measurement of Single-Top Cross Section and Test of Anomalous Wtb Coupling
}

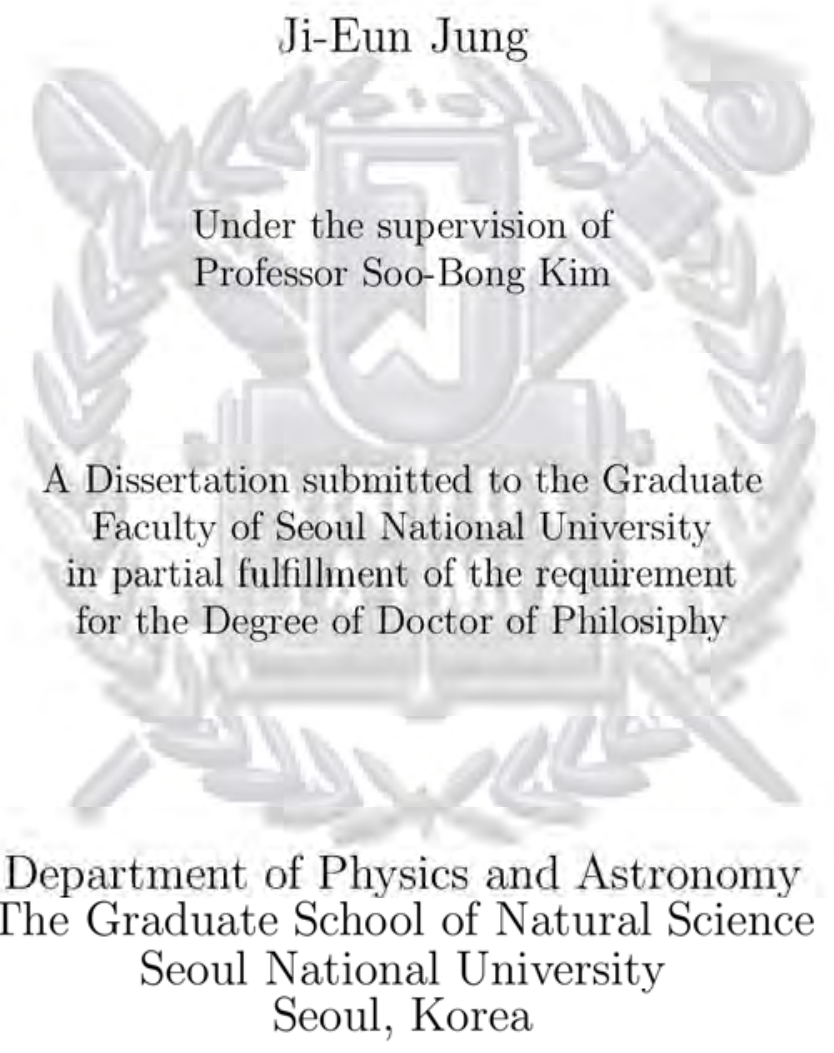

February 2010 


\begin{abstract}
The top quark is most often produced in $t \bar{t}$ pairs via the strong interaction, however electroweak production of a singly-produced top quark is also possible. Electroweak single-top production is more difficult to observe than $t \bar{t}$ production. Studying single-top production is important for the following reasons. It provides direct measurement of the CKM matrix element and also single-top events are a background to several searches for SM or non-SM signals, such as Higgs boson searches. The information of spin polarization of top-quark can be used to test anomalous W-t-b coupling.

This thesis describes the result of a measurement of single-top cross-section and a test of anomalous W-t-b coupling using $4.8 \mathrm{f} \mathrm{b}^{-1}$ of data collected by the CDF Run II experiment at the Fermilab Tevatron.

The measured cross-section is $1.83_{-0.6}^{+0.7} \mathrm{pb}$ and measured limit of $\mid V$ tb is 0.41 at $95 \%$ CL. The fraction of $V+$ A coupling is $0 \pm 28(\%)$.
\end{abstract}




\section{Contents}

1 Introduction 1

1.1 The Standard Model of Particle Physics . . . . . . . . . . . . . 1

1.2 Singly-produced Top Quark . . . . . . . . . . . . . . . 3

1.3 A test of Anomalous Wtb Coupling . . . . . . . . . 7

2 Experimental Apparatus 12

2.1 Accelerator . . . . . . . . . . . . . . . . . . . . . 12

2.2 Detector. Upgrade of RunII Detector . . . . . . . . . . . . . . 14

2.3 Trigger and Data Acquisition $\ldots \ldots \ldots \ldots \ldots \ldots$

2.4 Offline Data Reconstruction . . . . . . . . . . . . . 27

3 Monte Carlo Simulation 30

3.1 Signal. s-channel, t-channel . . . . . . . . . . . . . . 30

3.2 Backgrounds . . . . . . . . . . . . . . . . . . 33

3.2 .1 Top-Antitop . . . . . . . . . . . . . . . . 33

$3.2 .2 \quad \mathrm{~W}+$ Heavy Flavor . . . . . . . . . . . . . . . . 33

3.2 .3 Mistags . . . . . . . . . . . . . . . . . . . 34

3.2 .4 Dibosons . . . . . . . . . . . . . . . . . . . . . 35

3.2 .5 Non-W . . . . . . . . . . . . . . . 36

3.3 MC Simulation of Single-top Production including NLO effect . . . 36

4 Data and Event Selection 39

4.1 Data Set . . . . . . . . . . . . . . . . . . . . . 39

4.2 Trigger. . . . . . . . . . . . . . . . . . . 40

4.3 Selection for W Candidates . . . . . . . . . . . . . . . . 41

4.3 .1 Lepton . . . . . . . . . . . . . . . . . . . . . . . . 41

4.3 .2 Neutrino. . . . . . . . . . . . . . . . . . . 43

4.4 Jet Reconstruction . . . . . . . . . . . . . . . . . . . . . 44

4.5 B Jet Idendification $\ldots \ldots \ldots \ldots . \ldots \ldots$

4.6 Data Reduction and Final Candidate Sample $\ldots \ldots \ldots \ldots .47$

i 
5 Likelihood Method 60

5.1 Likelihood Fuction . . . . . . . . . . . . . . . . . . . 60

5.2 Input variables . . . . . . . . . . . . . . 61

5.3 Likelihood Function $\ldots \ldots \ldots \ldots$. . . . . . . . . 81

6 Extraction of Signal

6.1 Maximum Likelihood Fit . . . . . . . . . . . . . . . . . . . 83

6.2 Background Estimation . . . . . . . . . . . . . . . 84

6.3 Systematic Uncertainties . . . . . . . . . . . . . . . . . . 85

6.3 .1 Rate Systematic Uncertainties . . . . . . . . . . . 85

6.3.2 Shape Systematic Uncertainties . . . . . . . . . . . . . 94

6.4 Measurement of Cross Section . . . . . . . . . . . . . . . . 97

6.5 Significance . . . . . . . . . . . . . . . . . . . 99

7 Search for Anomalous W tb Coupling 101

7.1 Anomalous Wtb Coupling . . . . . . . . . . . . . . . 101

7.2 Search Result . . . . . . . . . . . . . . . . . . . . . . 102

8 Summary of Results and Discussion 106

A Monitoring and Finding of Beam Position 108

A.1 Fitting Algorithm . . . . . . . . . . . . . . . . . 108

A.2 Plots of Beam Position . . . . . . . . . . . . . 111

B MC Simulation of Single-top Production including NLO effect 118

B.1 Generator Level . . . . . . . . . . . . . . . . . . . . . . . . . . 119

B.2 Reconstruction level . . . . . . . . . . . . . . . . . . 124

C Shape Systematic Uncertainty 134

C.1 Plots . . . . . . . . . . . . . . . . . . . . . . . . 134 


\section{List of Figures}

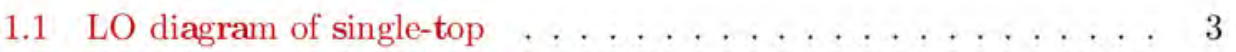

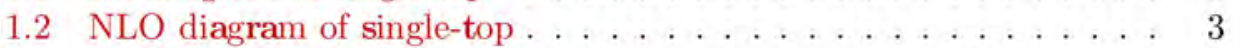

1.3 Top pair $(\mathrm{tt})$ Production . . . . . . . . . . . . . . . . . . . . 4

1.4 Vertex correction diagram. $Z \rightarrow \mathbf{b}$. . . . . . . . . . . . . 4

1.5 Single top quark cross section with center of mass energy . . . . . 6

1.6 Cross section of $\mathrm{pp}$ and $\mathrm{p} \overline{\mathrm{p}} \ldots \ldots \ldots \ldots \ldots \ldots$

1.7 Spin configuration for the top decay products . . . . . . . . 10

$1.8 \mathrm{~W}^{+}$decay for left-handed and right-handed helicity . . . . . . . . 10

1.9 The decay angles about top quark spin . . . . . . . . . . . . . 11

1.10 Angular correlations in the decay of a spin up top quark . . . . . . 11

2.1 Schematic overview of the Fermilab accelerator chain. . . . . . . . 13

2.2 The CDF Run II Detector . . . . . . . . . . . . . . . . . . . 15

2.3 Longitudinal View of the CDF II Tracking System . . . . . . . . . 16

2.4 Silicon micro-strip detector consists of Layer 00, SVX II and ISL. . 17

2.5 The SVX II 3 barrels . . . . . . . . . . . . . . . . . 18

2.6 The SVX II bulkhead design . . . . . . . . . . . . . . . . 19

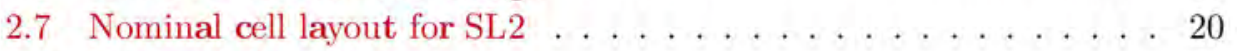

2.8 East endplate slots . . . . . . . . . . . . . . . . . 21

2.9 Calorimeter at CDF . . . . . . . . . . . . . . 24

2.10 Cross section of upper part of new end plug calorimeter . . . . . . 25

2.11 Geometrical Coverage of the Muon Chambers in $t-\phi$ plane . . . . 26

2.12 Fundamental block diagram of the CDF II data flow . . . . . . . . 28

3.1 2nd b-quark Pt distributions of $\mathrm{LO}$ and $\mathrm{NLO} \ldots . . . . . . .31$

3.2 2nd b-quark Pt distributions of LO and NLO in log scale . . . . . 32

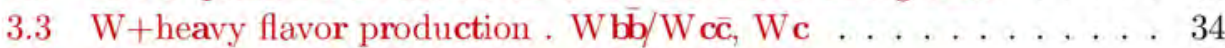

3.4 Diboson Production; WW, WZ, ZZ . . . . . . . . . . 35

4.1 Run II Integrated Luminosity . . . . . . . . . . . . . . . . . . 39

4.2 Primary and Secondary vertices of tracks of $\mid$ et ........ 47 
4.3 Lepton Pt. after selection cut . . . . . . . . . . . . . . . . 54

4.4 Missing Et. after selection cut . . . . . . . . . . . . . . 55

4.5 Tagged Jet Pt. after selection cut . . . . . . . . . . . . 56

4.6 Lepton E. after selection cut . . . . . . . . . . . . . . . 57

4.7 Neutrino E. after selection cut . . . . . . . . . . . . 58

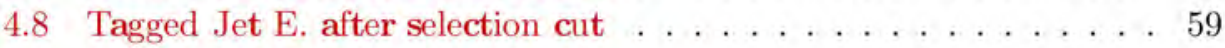

$5.1 \mathrm{Q} \cdot \%$. input variable in 2 et bin (norm) $\ldots \ldots \ldots \ldots \ldots . \ldots . \ldots . \ldots$

$5.2 \mathrm{Q} \cdot \eta$. input variable in 3 et bin (norm) . . . . . . . . 63

$5.3 \mathrm{M} \|$. input variable in 2 et bin (norm) $\ldots \ldots \ldots \ldots . \ldots 64$

$5.4 \mathrm{M}||$. input variable in 3 |et bin (norm) $\ldots \ldots \ldots \ldots . \ldots 64$

$5.5 \cos \theta$. input variable in 2 et bin (norm) $\ldots \ldots \ldots \ldots \ldots$

$5.6 \cos \theta$. input variable in 3 et bin (norm) . . . . . . . . 65

5.7 NN-btagger.input variable in 2 et bin (norm) $\ldots \ldots \ldots 66$

5.8 NN-btagger.input variable in 3 et bin (norm) $\ldots \ldots \ldots 6$

$5.9 \mathrm{H}_{T}$. input variable in 2 et bin (norm) $\ldots \ldots \ldots \ldots \ldots$

5.10 Matrix Element for t-channel. input variable in 2 et bin(norm) . . 68

$5.11 \chi^{2}$. input variable in 2 et bin (norm) $\ldots \ldots \ldots \ldots . \ldots 68$

5.12 Mtop. input variable in 3 et bin (norm) . . . . . . . . . 69

5.13 The number of b-tagged /ets in 3 et bin (norm) . . . . . . . . 69

$5.144 \mathrm{R}||$. input variable in $3 \mid$ et bin (norm) . . . . . . . . 70

$5.15 \mathrm{P}_{t}$ of the lowest $\mid$ et in $3 \mid$ et bin (norm) $\ldots \ldots \ldots \ldots \ldots$

$5.16 \mathrm{P}_{t}$ of $\mathbf{b} \mid$ et from top decay in 3 et bin (norm) $\ldots \ldots \ldots 71$

$5.17 \eta$ of $\mathrm{W}$. input variable in $3 \mid$ et bin (norm) . . . . . . . 71

5.18 NN-btagger.input variable in 2 et bin (stacked plot) . . . . . . 72

$5.19 \chi^{2}$. input variable in 2 et bin (stacked plot) . . . . . . . 72

$5.20 \log \left(\mathrm{ME}_{t-c h}\right)$. input variable in 2 et bin (stacked plot) $\ldots \ldots .73$

$5.21 \mathrm{H}_{T}$. input variable in 2 et bin (stacked plot) . . . . . . 73

$5.22 \mathrm{M} \mid$. input variable in 2 et bin (stacked plot) $\ldots \ldots \ldots \ldots .74$

$5.23 \cos \theta$. input variable in 2 et bin (stacked plot) $\ldots \ldots \ldots \ldots 74$

$5.24 \mathrm{Q} \cdot \eta$. input variable in 2 et bin (stacked plot) $\ldots \ldots \ldots \ldots 75$

5.25 NN-btagger.input variable in 3 et bin (stacked plot) . . . . . 75

$5.26 \cos \theta$. input variable in 3 et bin (stacked plot) . . . . . . 76

$5.274 \mathrm{R} \mid$. . input variable in 3 |et bin (stacked plot) . . . . . . . 76

5.28 Lowest Et of |et . input variable in 3 |et bin (stacked plot) . . . . 77

$5.29 \eta$ of $\mathrm{W}$. input variable in 3 et bin (stacked plot) . . . . . . 77

$5.30 \mathrm{M}||$ not choson to be $\mathbf{b}$ from top in 3 et bin (stacked plot) . . . . 78

5.31 Mtop. input variable in 3 et bin (stacked plot) $\ldots \ldots \ldots \ldots 78$

5.32 The number of b-tagged |ets in 3 |et bin (stacked plot) . . . . . 79

$5.33 \mathrm{P}_{t}$ of the lowest $\mid$ et in $3 \mid$ et bin (stacked plot) $\ldots \ldots \ldots \ldots 79$

$5.34 \mathrm{Q} \cdot \%$. input variable in 3 et bin (stacked plot) . . . . . 80 
5.352 2|et bin Likelihood function . . . . . . . . . . . . . . . . 81

5.363 |et bin Likelihood function . . . . . . . . . . . . . . . . 82

6.1 The Uncertainty of JES $\ldots \ldots \ldots \ldots \ldots \ldots$

6.2 KF Template fits to the $\mathrm{W}+1$ et bin . . . . . . . . 88

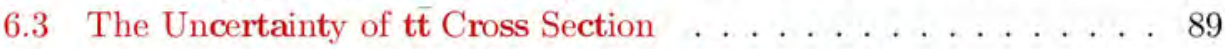

6.4 The Uncertianty of Initial State Radiation . . . . . . . . . . . . . 90

6.5 The Comparison of the Parton Distribution Functions . . . . . . . 91

6.6 JES Shape Systematic Uncertainty(s-/t-channel) $\ldots \ldots \ldots \ldots .95$

6.7 JES Shape Systematic Uncertainty (ttbar/Wbb) . . . . . . . . . 96

6.8 Measured cross-section using $4.8^{-1} \ldots \ldots \ldots \ldots \ldots$. . . . . 97

6.9 Extraction of limits on $\mid V \mathrm{tb}^{2} \ldots \ldots \ldots \ldots \ldots \ldots \ldots$

6.10 plot of $-\ln \mathrm{Q}$ for p-value . . . . . . . . . . . . . . 100

7.1 The RRLL sample's vertex coupling choices . . . . . . . . . 102

$7.2 \cos \theta$ at the HEPG level . . . . . . . . . . . . . . 103

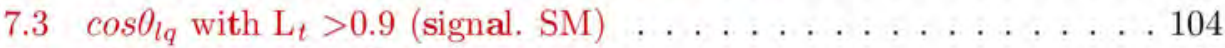

7.4 2D Cross-section Fit . . . . . . . . . . . . . . 105

A.1 SVX beam position in xz-plane . . . . . . . . . . . 108

A.2 SVX beam position in yz-plane . . . . . . . . . . 109

A.3 Fit Codes for SVX . . . . . . . . . . . . . . . . . 111

A.4 Fit Codes for COT . . . . . . . . . . . . . . . . . 112

A.5 BeamX Position of SVX . . . . . . . . . . . . 113

A.6 BeamX Position of COT . . . . . . . . . . . . . . 114

A. 7 Difference of SVX and COT beamline . . . . . . . . 115

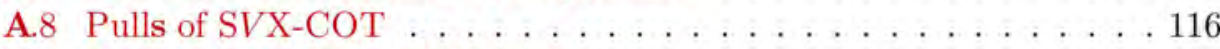

A.9 Pull distribution of SVX-COT $\ldots \ldots \ldots \ldots \ldots \ldots \ldots \ldots$

B.1 s-channel sample. Top mass . . . . . . . . . . . . . . . . . . . 119

B.2 s-channel sample. Lepton . . . . . . . . . . . . . . . . 120

B.3 s-channel sample. Neutrino . . . . . . . . . . . . . . . 120

B.4 s-channel sample. b-quark from top decay . . . . . . . . . . . . 121

B.5 s-channel sample. Top quark . . . . . . . . . . . . . . . . 121

B.6 t-channel sample. Top mass . . . . . . . . . . . . . . . . . . 122

B.7 t-channel sample. Lepton . . . . . . . . . . . . . . . . . . 122

B.8 t-channel sample. Neutrino . . . . . . . . . . . . . . . . 123

B.9 t-channel sample, b-quark from top decay . . . . . . . . . . . 123

B.10 s-channel sample. Top mass and $\mathrm{Q} \times \eta \ldots \ldots \ldots . \ldots 126$

B.11 s-channel sample. Lepton . . . . . . . . . . . . . . . . . . 127

B.12 s-channel sample. Neutrino . . . . . . . . . . . . . 128

B.13 s-channel sample. b-quark from top decay . . . . . . . . . 128 
B.14 s-channel sample. b-quark not from top decay . . . . . . . . . 129

B.15 s-channel sample. Top quark . . . . . . . . . . . . . . . . . 129

B.16 t-channel sample. Top mass and $\mathrm{Q} \times \eta \ldots \ldots \ldots \ldots \ldots$

B.17 t-channel sample. Lepton . . . . . . . . . . . . . . . . . 131

B.18 t-channel sample. Neutrino . . . . . . . . . . . . . . 131

B.19 t-channel sample. b-quark from top decay . . . . . . . . . . . 132

B.20 t-channel sample. Light quark . . . . . . . . . . . . . . 132

B.21 t-channel sample. Top quark . . . . . . . . . . . . . 133

C.1 JES Shape Systematic Uncertainty(s-channel) . . . . . . . . . . . 134

C.2 JES Shape Systematic Uncertainty(t-channel) . . . . . . . . . . 135

C.3 JES Shape Systematic Uncertainty (ttbar/Wbb) . . . . . . . . . 135

C.4 JES Shape Systematic Uncertainty (WC/Z|ets) $\ldots \ldots \ldots \ldots . .136$

C.5 ISR Shape Systematic Uncertainty (s-/t-channel) . . . . . . . . 137

C.6 ISR Shape Systematic Uncertainty(s-/t-channel) . . . . . . . . . 138

C.7 FSR Shape Systematic Uncertainty(s-/t-channel) . . . . . . . . . 139

C.8 FSR Shape Systematic Uncertainty(s-/t-channel) . . . . . . . . 139

C.9 Eta|2 Shape Systematic Uncertainty(s-/t-channel) . . . . . . . 140

C.10 Eta 2 Shape Systematic Uncertainty(ttbar) . . . . . . . . . . . 140

C.11 Eta 2 Shape Systematic Uncertainty $(\mathrm{Wbb} / \mathrm{WC}) \quad \ldots . . . . . .141$

C.12 Eta 2 Shape Systematic Uncertainty $(\operatorname{mistag} / Z \mid$ ets $) \ldots . . . . .142$

C.13 DeltaR|2 Shape Systematic Uncertainty(s-/t-channel) . . . . . . 143

C.14 DeltaR|2 Shape Systematic Uncertainty(ttbar) . . . . . . . . 143

C.15 DeltaR|2 Shape Systematic Uncertainty(Wbb/WC) . . . . . . . . 144

C.16 DeltaR $\mid 2$ Shape Systematic Uncertainty (mistag/Z/ets) . . . . . 145

C.17 NNbtag Shape Systematic Uncertainty(Diboson/WC) . . . . . 146

C.18 Nonwflav Shape Systematic Uncertainty(nonW) . . . . . . . . 146

vi 


\section{List of Tables}

1.1 Elementary Particles in Standard Model . . . . . . . . . . . . . . 2

1.2 The cross section of single-top production at Tevatron and LHC . 5

1.3 The fractional cross section for $\mathrm{W}^{*}$ channel . . . . . . . . . . 9

1.4 The fractional cross section for $\mathrm{Wg}$-fusion channel $\ldots . . . . . .99$

2.1 Silicon detector mechanical dimensions . . . . . . . . . . . . 18

2.2 COT Mechanical Summary . . . . . . . . . . . . . . . 22

$3.1 \mathrm{P}_{T}$ and $\mathrm{P}_{Z}$ for MadEvent and MC@NLO at hepg level . . . . . 37

$3.2 \mathrm{P}_{T}$ and $\mathrm{P}_{Z}$ for MadEvent and MC@NLO samples at rec level . . . 38

4.1 CDF Dataset Summary . . . . . . . . . . . . . . . . 40

4.2 Cental Electron Identification Requirements . . . . . . . . . . . . . . 42

4.3 Plug Electron Identification Requirements . . . . . . . . . . . . . . . . 42

4.4 Muon Identification Requirements . . . . . . . . . . . . . . . 44

4.5 CEM Acceptance Table of s-channel Sample(stop00) . . . . . . . . 49

4.6 PHX Acceptance Table of s-channel Sample(stop00) . . . . . . . . 49

4.7 CMUP Acceptance Table of s-channel Sample(stop00) . . . . . . . 50

4.8 CMX Acceptance Table of s-channel Sample(stop00) . . . . . . . . 50

4.9 CEM Acceptance Table of Wbb Sample(btop0w) . . . . . . . . . . 51

4.10 PHX Acceptance Table of Wbb Sample(btop0w) . . . . . . . . . . 51

4.11 CMUP Acceptance Table of Wbb Sample(btop0w) . . . . . . . . . 52

4.12 CMX Acceptance Table of Wbb Sample(btop0w) . . . . . . . . . . 52

4.13 Data and Background for single-top production with $4.8 \mathrm{fb}^{-1} \ldots . .53$

6.1 Data and Background Prediction . . . . . . . . . . . . 85

6.2 Uncertainty of Luminosity from Various Sources . . . . . . . . . . 89

6.3 Systematic rate Uncertainties and Error . . . . . . . . . . . . . . . 91

6.4 Systematic rate uncertainties for 2 - et . . . . . . . . . . . . . . . . . . . . . . . . 92

6.5 Systematic rate uncertainties for 3 - et . . . . . . . . . . . 93

B.1 Acceptance table. s-channel MadEvent . . . . . . . . . . . . . 124

vii 
B.2 Acceptance table. s-channel MC@NLO . . . . . . . . . . . . . 125

B.3 Acceptance table. t-channel MadEvent . . . . . . . . . . . . . 125

B.4 Acceptance table. t-channel MC@NLO . . . . . . . . . . . . 127

viii 


\section{Chapter 1}

\section{Introduction}

\subsection{The Standard Model of Particle Physics}

The particle physics treat the constituents of matter and the nature of the interaction between them. The Standard Model(SM) explains successfully the particles and their interactions in universe by gauge theories. There are four interaction forces of the weak nuclear force, the electromagnetic force, the strong nuclear force, and gravitation. The weak, electomagnetic and strong forces are combined in Standard Model theory, but the gravitation is not yet described by the SM.

One model of the particles is the spin $1 / 2$ fermions, that is the quarks and leptons. There are 6 quarks of $u p(u)$ and down(d), charm(c) and strange(s), and top(t) and bottom(b), with fractional charge of $+\frac{2}{3} e$ and $-\frac{1}{3} e$. The baryons are bound states of three quarks and the mesons are composed of a quark and an antiquark; the quarks don't exist as a free particle. There are three leptons(electron, muon and tau) with the charge of $-1 e$ and three neutrinos(no charge) associated with its leptons. Originally, neutrino mass is assumed massless by SM, but from the recent experimental result, the evidence that neutrino has non-zero mass is provided. The neutrino interact only through the weak force and the leptons interact additionally through the electromagnetic force.

The another model of particles is boson, which has integral spin. The interactions between particles can be occured as two particles exchange a boson.

The list of elementary particles in Standard Model is shown in Table 1.1.

The weak interaction is mediated by the bosons, charged $\mathrm{W}^{ \pm}$boson or neutral $Z^{0}$ boson. The weak interactions are processed in short distances because the bosons are massive; the Ws have measured mass of $80.4 \mathrm{GeV} / \mathrm{c}^{2}$ and $Z^{0}$ has a measured mass of $91.2 \mathrm{GeV} / \mathbf{c}^{2}$. The transition between quarks of different flavors can occur via the weak interaction. Cabibbo-Kobayashi-Maskawa(CKM) matrix 


\begin{tabular}{|c|c|c|c|c|c|}
\hline \multicolumn{4}{|c|}{ Fermions } & Charge & Spin \\
\hline Quarks & $\left(\begin{array}{l}u \\
d\end{array}\right)$ & $\left(\begin{array}{l}\mathrm{c} \\
\mathrm{s}\end{array}\right)$ & $\left(\begin{array}{l}t \\
b\end{array}\right)$ & $\begin{array}{l}+\frac{2}{3} \mathrm{e} \\
-\frac{1}{3} \mathrm{e}\end{array}$ & $\begin{array}{l}\frac{1}{2} \\
\frac{1}{2}\end{array}$ \\
\hline Leptons & $\left(\begin{array}{c}\mathrm{e} \\
\nu_{e}\end{array}\right)$ & $\left(\begin{array}{c}\mu \\
\nu_{\mu}\end{array}\right)$ & $\left(\begin{array}{c}\tau \\
\nu_{\tau}\end{array}\right)$ & $\begin{array}{c}-1 e \\
0\end{array}$ & $\begin{array}{l}\frac{1}{2} \\
\frac{1}{2}\end{array}$ \\
\hline \multicolumn{4}{|c|}{ Gauge Bosons } & Charge & Spin \\
\hline \multicolumn{4}{|c|}{$\begin{array}{c}\gamma \text { (photon) } \\
\mathrm{W}^{ \pm}, Z \text { (weak boson) } \\
\mathrm{g} \text { (gluon) }\end{array}$} & $\begin{array}{c}0 \\
\pm 1 \\
0\end{array}$ & $\begin{array}{l}1 \\
1 \\
1\end{array}$ \\
\hline
\end{tabular}

Table 1.1. Elementary Particles in Standard Model

represents the strenth of this transition of quarks.

The CKM matrix, $3 \times 3$ unitary matrix, is represented.

$$
\left(\begin{array}{c}
\mathrm{d}^{\prime} \\
\mathbf{s}^{\prime} \\
\mathbf{b}^{\prime}
\end{array}\right) /\left(\begin{array}{lll}
V_{u d} & V_{u s} & V_{u b} \\
V_{c d} & V_{c s} & V_{c b} \\
V_{t d} & V_{l s} & V_{t b}
\end{array}\right)\left(\begin{array}{c}
\mathrm{d} \\
\mathbf{s} \\
\mathbf{b}
\end{array}\right)
$$

where $V_{C K M}$ is obtained using the method of Refs. [0],

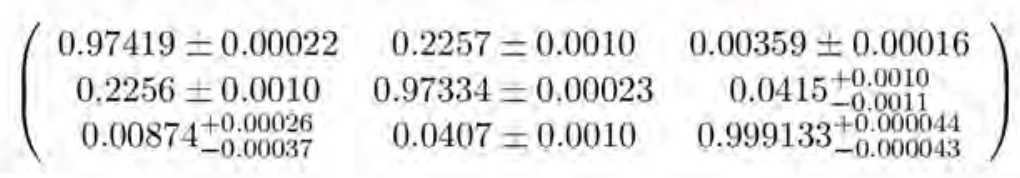

Quantum Electrodynamics describes the electromagnetic interaction among the quarks and leptons through the exchange of massless spin-1 photons. This electomagnetic force reach to the infinite range due to the massless photon. The electroweak theory is the unification of the electromagnetic and weak interaction in the Standard Model.

Quantum Chromo Dynamics(QCD) describes the strong interaction via gluon exchange. The spin- 1 gluons is massless and carries color charge $(R, G, B)$ affecting gluon self-interaction. The quarks also have the color charge and they are bound via the strong interaction forming colorless states(baryon or meson). From this feature, the strong force increases at large distance and decreases at short distances, which is asymptotic freedom. 


\subsection{Singly-produced Top Quark}

In proton-antiproton $(p \mathrm{p})$ collisions at $1.96 \mathrm{TeV}$, top quarks are predominantly produced in $t \bar{t}$ pairs via strong interaction processes. The top quark in top pair production was discovered in 1995 at the Tevatron. Singly-produced-top quarks are also expected via the electroweak interaction involving a Wtb vertex in the Standard Model (SM).

The single-top-quarks can be produced through three partonic processes. the s-channel production $q \bar{q}^{0} \rightarrow \mathrm{tb}$, called $\mathrm{W}^{*}$ production, the t-channel production $\mathrm{qg} \rightarrow \mathrm{tbq}^{0}$, called Wg-fusion, and associated production with a W boson $\mathrm{bg} \rightarrow \mathrm{tW}$. At the Tevatron the t-channel and s-channel process are dominant, while associated tW production is negligible $\left(\sigma_{s} / 0.88=0.05 \mathrm{pb}\right.$ (s-channel), $\sigma_{t} / 1.98$ $\pm 0.08 \mathrm{pb}$ (t-channel) in SM). Therefore, the s-channel and t-channel are only considered in this analysis. The leading-order $(\mathrm{LO})$ and the next-to-reading order(NLO) Feynman diagrams for the single-top production channels are shown in Figure 1.1 and Figure 1.2 respectively.
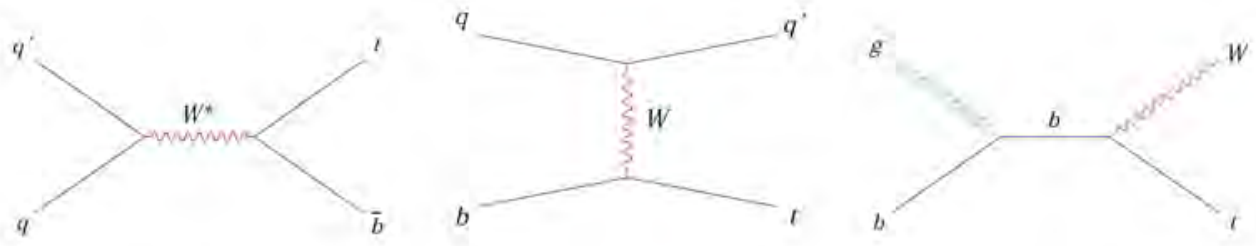

Figure 1.1. LO diagram for s-channel(left), t-channel(middle) and associated tW(right) single-top-quark production
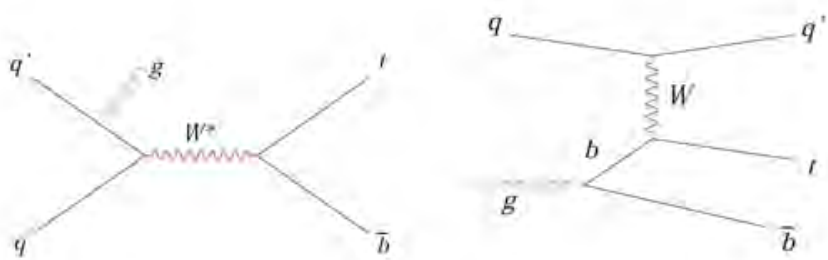

Figure 1.2. Examples of NLO diagrams for s-channel(left) and t-channel(right) single-top-quark production 
Electroweak single-top-quark production via a Wtb vertex gives unique opportunity of the direct measurement of the CKM(Cabibbo-Kobayashi-Maskawa) matrix element $\left|V_{t b}\right|$. The cross section is proportional to the $\left|V_{t b}\right|^{2}$ and this value can be extracted from the measurement of single-top cross-section.

$\left|V_{t b}\right|$ characterizes the strength of transition of a top quark into a bottom quark when the two particles interact through the electroweak interaction. The quantity is currently not well measured and all previous results were under the assumption that the CKM matrix is unitary. In t t production (Fig. 1.3), the determination of $\left|V_{t b}\right|$ from top decays uses the ratio of braching fractions $\mathrm{R} / \mathrm{B}(\mathbf{t} \rightarrow \mathrm{Wb}) / \mathrm{B}(\mathbf{t} \rightarrow \mathrm{Wq}) /\left|V_{t b}\right|^{2} /\left(\sum_{q}\left|V_{t q}\right|^{2}\right)$, where q / $\mathbf{b} \mathbf{s} ; \mathrm{d}$. The CDF and D0 measurement performed on data collected during Run II of the Tevatron give $\left|V_{t b}\right|>0.78[1]$ and $\left|V_{t b}\right|>0.89$ [2]. For another examples, the result from top-loop contributions of $(\Gamma \rightarrow \mathbf{b})$ is $\left|V_{t b}\right| / 0.77_{-0.24}^{+0.18}[$ [3]. The Vertex diagram shown at the one-loop level in Fig. 1.4 which affects the $Z$ partial width, $\Gamma_{b \tilde{b}}$
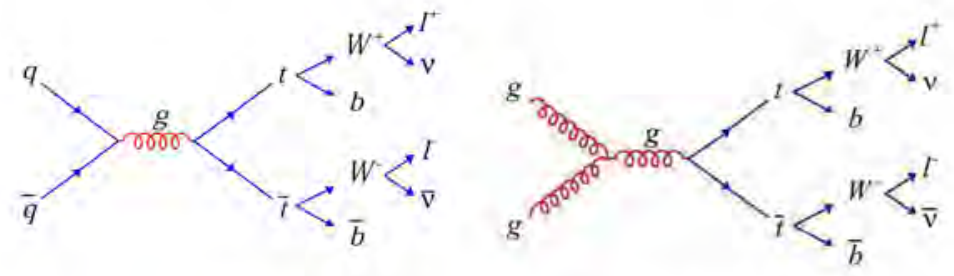

Figure 1.3. Top pair $(\mathbf{t} \overline{\mathbf{t}})$ Production
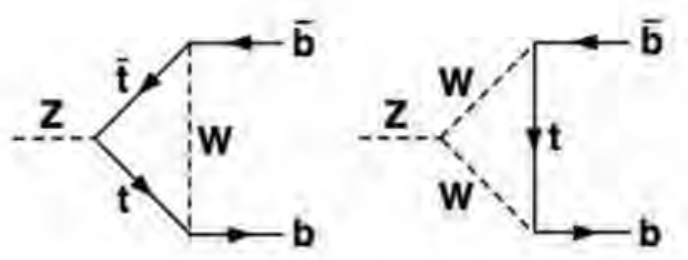

Figure 1.4. Vertex correction diagrams, at order of one-loop, which contribute to the partial width for $Z \rightarrow \mathbf{b} \mathbf{b}$

Measuring the single top quark production cross section allows us to avoid the assumption of unitarity, to measure $\left|V_{t b}\right|$ precisely, and to draw conclusions about a hypothetical fourth generation of quarks. Studying the polarization in single top events will be a very important test of the weak interaction of the top 
quark. Finally, measuring the single top process tests exotic models beyond the Standard Model, which predict anomalously altered single top producton rates like heavy right handed W' bosons, flavor changing neutral currents or large extra dimensions.

The CDF and D0 collaborations have sought single-top production for over a decade. Evidence of single-top production was published [8][9][10] and finally both collaborations observed single top production at the $5 \sigma$ level of significance $[6][7]$.

Table 1.2 shows expected cross section of single-top production at Tevatron(1.8 $\mathrm{TeV}$ (Run I), 1.96TeV (Run II)) and $\mathrm{LHC}(14 \mathrm{TeV})$.

\begin{tabular}{|c|c|c|c|}
\hline Procerss & $\overline{\mathrm{S}}$ & $\sigma_{L O}(\mathrm{pb})$ & $\sigma_{N L O}(\mathrm{pb})$ \\
\hline \multirow[t]{5}{*}{ s-channel } & $1.8 \mathrm{Te} V \mathrm{pp}(\mathbf{t})$ & 0.259 & $0.380=0.002$ \\
\hline & $1.96 \mathrm{TeV} \mathrm{p} \overline{\mathrm{p}}(\mathbf{t})$ & 0.304 & $0.447 \pm 0.002$ \\
\hline & $2 \mathrm{Te} V \mathrm{pp}(\mathbf{t})$ & 0.315 & $0.463 \pm 0.002$ \\
\hline & $14 \mathrm{Te} V \mathrm{pp}(\mathbf{t})$ & 4.53 & $6.55=0.03$ \\
\hline & $14 \mathrm{Te} V \mathrm{pp}(\overline{\mathbf{t}})$ & 2.74 & $4.07=0.02$ \\
\hline \multirow[t]{5}{*}{ t-channel } & $1.8 \mathrm{Te} V \mathrm{p} \overline{\mathrm{p}}(\mathbf{t})$ & 0.648 & $0.702=0.003$ \\
\hline & $1.96 \mathrm{Te} V \mathrm{p \overline {p }}(\mathbf{t})$ & 0.883 & $0.959 \pm 0.002$ \\
\hline & $2 \mathrm{TeV}$ p $\bar{p}(\mathbf{t})$ & 0.948 & $1.029 \pm 0.004$ \\
\hline & $14 \mathrm{TeV} \mathrm{pp}(\mathbf{t})$ & 144.8 & $152.6=0.6$ \\
\hline & $14 \mathrm{Te} V \mathrm{pp}(\overline{\mathbf{t}})$ & 83.4 & $90.0 \pm 0.5$ \\
\hline
\end{tabular}

Table 1.2. The cross section of single-top production at Tevatron and LHC

Figure 1.5 shows the graph of single-top cross section versus center of mass energy. The cross-section of both s-channel and t-channel are growing by increase of the center of mass energy. This is consistent with expectation that increase of initial energy corresponds to larger production cross section. The cross section of single-top production can be calculated as follows.

Consider the process $\mathrm{p}_{1}+\mathrm{p}_{2} \rightarrow \mathrm{p}_{3}+\mathrm{p}_{4}$, where particle's momentum [A]. The partonic kinematical invariants are $\mathbf{s} /\left(\mathrm{p}_{1}+\mathrm{p}_{2}\right)^{2}, \mathbf{t} /\left(\mathrm{p}_{1}-\mathrm{p}_{3}\right)^{2}, \mathrm{u} /\left(\mathrm{p}_{2}-\mathrm{p}_{3}\right)^{2}$, $\mathbf{s}_{4} / \mathbf{s}+\mathbf{t}+\mathrm{u}-\mathrm{m}_{3}-\mathrm{m}_{4}$. The hadronic kinematical invariants are $\mathrm{S} /\left(\mathrm{p}_{h 1}+\mathrm{ph} 2\right)^{2}$, $\mathrm{T} /\left(\mathrm{p}_{h 1}-\mathrm{p}_{3}\right)^{2}, \mathrm{U} /\left(\mathrm{p}_{h 2}-\mathrm{p}_{3}\right)^{2}, \mathrm{~S}_{4} / \mathrm{S}+\mathrm{T}+\mathrm{U}-\mathrm{m}_{3}^{2}-\mathrm{m}_{4}^{2}$, where $\mathrm{h}_{1}$ and $\mathrm{h}_{2}$ are the hadrons (protons and antiprotons at the Tevatron) corresponding to partons $\mathrm{p}_{1}$ and $\mathrm{p}_{2}$; thus, $\mathrm{p}_{1} / \mathrm{x}_{1} \mathrm{p}_{h 1}$ and $\mathrm{p}_{2} / \mathrm{x}_{2} \mathrm{p}_{h 2}$. The relations are as follows. $\mathrm{s} / \mathrm{x}_{1} \mathrm{x}_{2} \mathrm{~S} ; \mathrm{t}-\mathrm{m}_{3}^{2} / \mathrm{x}_{1}\left(\mathrm{~T}-\mathrm{m}_{3}^{2}\right) ; \mathrm{u}-\mathrm{m}_{3}^{2} / \mathrm{x}_{2}\left(\mathrm{U}-\mathrm{m}_{3}^{2}\right)$.

The hadron-level cross section $\sigma$ is

$$
\left.\sigma / \int \mathrm{dx}_{1} \mathrm{dx}_{2} \phi\left(\mathrm{x}_{1}\right) \phi\left(\mathrm{x}_{2}\right) \hat{\sigma} /\right) \frac{d \sigma}{\mathrm{dTdU}}
$$




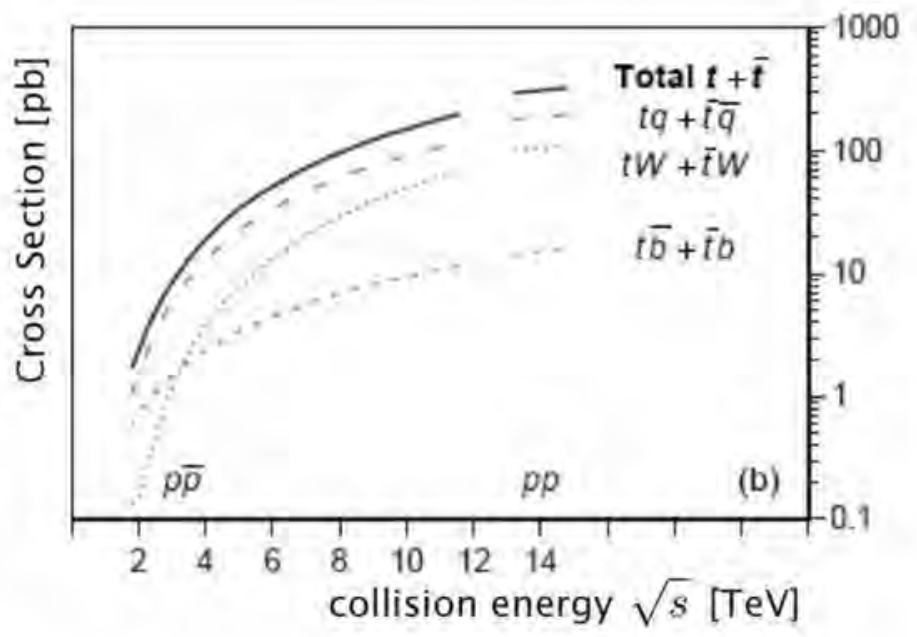

Figure 1.5. Single top quark cross section with center of mass energy

$$
/ \int \mathrm{dx}_{1} \mathrm{dx}_{2} \frac{\mathrm{dt}}{\mathrm{dT}} \frac{\mathrm{du}}{\mathrm{dU}} \phi\left(\mathrm{x}_{1}\right) \phi\left(\mathrm{x}_{2}\right) \frac{\mathrm{d} \hat{\sigma}}{\mathrm{dtdu}}
$$

where $\hat{\sigma}$ is parton-level cross section, $\phi(\mathrm{x})$ is the parton distribution.

Now $\mathrm{dt} / \mathrm{x}_{1} \mathrm{dT}$ and $\mathrm{du} / \mathrm{x}_{2} \mathrm{dU}$,

$$
\begin{aligned}
\left.\frac{d \sigma}{\mathrm{dTdU}} / \int \mathrm{dx}_{1} \mathrm{dx}_{2} \mathrm{x}_{1} \mathrm{x}_{2} \phi\left(\mathrm{x}_{1}\right) \phi\left(\mathrm{x}_{2}\right) \frac{\mathrm{d} \hat{\sigma}}{\mathrm{dtdu}} /\right) \sigma \\
\qquad \int \mathrm{dTdUdx}_{1} \mathrm{dx}_{2} \mathrm{ds}_{4} \frac{\mathrm{x}_{1} \mathrm{x}_{2}}{\mathrm{x}_{2} \mathrm{~S}+\mathrm{T}-\mathrm{m}_{3}^{2}} \phi\left(\mathrm{x}_{1}\right) \phi\left(\mathrm{x}_{2}\right) \frac{\mathrm{d} \hat{\sigma}}{\mathrm{d} \mathbf{t d u}} .
\end{aligned}
$$

The Born-level differential partonic cross section is written as

$$
\frac{\mathrm{d}^{2} \hat{\sigma}_{p_{1} p_{2} \rightarrow p_{3} p_{4}}^{B}}{\mathrm{~d} \mathrm{t} d \mathrm{u}} / \mathrm{F}_{p_{1} p_{2} \rightarrow p_{3} p_{4}}^{B} \delta\left(\mathbf{s}_{4}\right) / \frac{\left|\mathrm{M}_{p_{1} p_{2} p_{3} p_{4}}\right|^{2}}{16 \pi \mathbf{s}^{2}} \delta\left(\mathbf{s}_{4}\right) .
$$

For the t-channel process $u b \rightarrow d t$,

$$
\left|\mathrm{M}_{u b \rightarrow d t}\right|^{2} / \frac{4 \pi^{2} \alpha^{2} V_{t b}^{2} V_{u d}^{2}}{\sin ^{4} \theta_{W}} \frac{\mathbf{s}\left(\mathbf{s}-\mathbf{m}_{t}^{2}\right)}{\left(\mathbf{t}-\mathrm{m}_{W}^{2}\right)^{2}} .
$$

For the t-channel process $\mathrm{db} \rightarrow \bar{u} t$,

$$
\left|\mathrm{M}_{\bar{d} b \rightarrow \bar{u} t}\right|^{2} / \frac{4 \phi^{2} \alpha^{2} V_{t b}^{2} V_{u d}^{2}}{\sin ^{4} \theta_{W}\left(\mathbf{t}-\mathrm{m}_{W}^{2}\right)^{2}}\left[(\mathbf{s}+\mathbf{t})^{2}-(\mathbf{s}-\mathbf{t}) \mathrm{m}_{t}^{2}\right] .
$$


For the s-channel process u $\overline{\mathrm{d}} \rightarrow \overline{\mathbf{b}}$,

$$
\left|\mathrm{M}_{u \bar{d} \rightarrow \bar{b} t}\right|^{2} / \frac{4 \pi^{2} \alpha^{2} V_{t b}^{2} V_{u d}^{2}}{\sin ^{4} \theta_{W}} \frac{\mathbf{t}\left(\mathrm{t}-\mathrm{m}_{t}^{2}\right)}{\left(\mathrm{s}-\mathrm{m}_{W}^{2}\right)^{2}}
$$

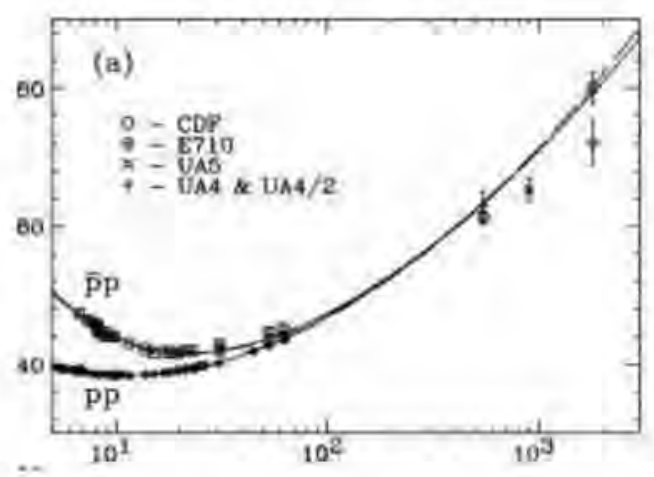

Figure 1.6. Cross section of pp and pp̄

Suppose b quark content due to gluon spitting is enough large, the t-channel cross section is larger than s-channel. This is because the kinematic dependence of $\mathbf{t}$-channel is $\left(\mathbf{s}-\mathrm{m}_{1}^{2}\right) / \mathbf{s}\left(\mathrm{t}-\mathrm{m}_{W}^{2}\right)^{2}$ while kinematic dependence of s-channel is $\mathbf{t}\left(\mathbf{t}-\mathrm{m}_{t}^{2}\right) / \mathbf{s}^{2}\left(\mathbf{s}-\mathrm{m}_{W}^{2}\right)^{2}$. This means that $\mathbf{t}$-channel is more kinematically probable than s-channel for large $\mathbf{s}$. As small $\mathbf{s}$, the $\mathbf{b}$ quark content in proton is much smaller than that of light quark, and thus the cross section of s-channel is larger. However, the $\mathbf{b}$ quark content becomes larger for high energy(i.e. larger s), and thus t-channel cross section becomes larger at high $\mathbf{s}$.

\subsection{A test of Anomalous Wtb Coupling}

The electroweakly produced top quarks are nearly $100 \%$ polarized. The top quark decay width is much larger than $\Lambda_{\mathrm{QCD}}$ [11], so the top quarks remain polarized when they decay. The decay of top quark proceeds so rapidly that toponium bound states and T mesons do not have time to form, so the decay products of the top quark are correlated with its spin. On the other hand, the bound state mesons(or lighter quarks) such as the $\mathrm{B}^{0}$ and the $\mathrm{B}^{ \pm}$lost the information of their polarization due to spin-exchahging interactions with the spectator quarks and gluons. 
The polarization fraction of singly-produced top quarks is expected to be modified if the top quark couplings to the $\mathrm{SM} \mathrm{W}^{ \pm}$are anomalous, if the production $\mathrm{W}$ has an admixture of an exotic $\mathrm{W}^{0}$, if single-top quarks are produced in addition to the SM mechanisms by more exotic mechanisms [17] such as FCNC's [16], charged Higgs boson exchange instead of SM W exchange in the production channel.

The meausured cross-section of SM single-top production at CDF is $2.3_{-0.5}^{+0.6} \mathrm{pb}$ and this measured value is lower than the SM prediction of $2.864 \pm 0.33$ [25][26]. Then any fraction of the single top signal escaped our detecton may exist. Becuase in our analysis the input variables (decay angles and matrix elements and so on) are used to separate signal from background, top quarks produced with right-handed polarization would not look as signal-like as top quarks with lefthanded polarization.

At this point, we focused to the anomalous Wtb coupling in single-top production. As it is mentioned before, single top production allow a direct measurement of the CKM matrix element $\left|V_{t b}\right|$. We can test the anomalous Wtb coupling by using angular correlations in the events due to the top spin correlation.

The matrix element for the electroweak process $u \bar{d} \rightarrow$ tb via virtual W boson can be descriped as follows [11].

As the production of a spin up top quark,

$$
\left|\mathrm{M}\left(0 \rightarrow \overline{\mathrm{u}} \mathrm{dt} \mathbf{t}_{\uparrow} \overline{\mathbf{b}}\right)\right|^{2} / \mathrm{g}_{W}^{4}\left|V_{u d}\right|^{2} \mathrm{~N}_{c}^{2} \frac{\left(2 \mathrm{~d} \cdot \mathbf{t}_{2}\right)(2 \mathrm{u} \cdot \mathrm{b})}{\left(2 \mathrm{u} \cdot \mathrm{d}-\mathrm{m}_{W}^{2}\right)^{2}+\left(\mathrm{m}_{W} \Gamma_{W}\right)^{2}} .
$$

As the production of a spin down top quark,

$$
\left|\mathrm{M}\left(0 \rightarrow \overline{\mathrm{udt}} \mathbf{t}_{\downarrow} \mathbf{b}\right)\right|^{2} / \mathrm{g}_{W}^{4}\left|V_{u d}\right|^{2} \mathrm{~N}_{c}^{2} \frac{\left(2 \mathrm{~d} \cdot \mathbf{t}_{1}\right)(2 \mathrm{u} \cdot \mathrm{b})}{\left(2 \mathrm{u} \cdot \mathrm{d}-\mathrm{m}_{W}^{2}\right)^{2}+\left(\mathrm{m}_{W} \Gamma_{W}\right)^{2}}
$$

where top quark momentum is decomposed into a sum of two massless auxiliary momenta, $\mathbf{t}_{1} \equiv \frac{1}{2}\left(\mathbf{t}+\mathrm{m}_{t} \mathbf{s}\right), \mathbf{t}_{2} \equiv \frac{1}{2}\left(\mathbf{t}-\mathrm{m}_{l} \mathbf{s}\right)$ ( $\mathbf{s}$ is spin vector of the top quark) and $g_{W}$ is the weak coupling constant, $m_{W}$ and $\Gamma_{W}$ are mass and width of the W boson, $\mathrm{N}_{c}$ is the number of colors. As those equations, the top quark produced via the $\mathrm{W}^{*}$ process are $100 \%$ polarized along the direction of the d-type quark, since (1.2) vanishes if we choose $\mathbf{t}_{1} / \mathrm{d}$.

At Wg fusion process, the matrix element for the production of spin down top quarks in the basis where the spin axis coincides with the d quark direction.

$$
|\mathrm{M}(0 \rightarrow \overline{\mathrm{u}} \mathrm{dgtb})|^{2} / \frac{\mathrm{g}_{W}^{4} \mathrm{~g}_{s}^{2}\left|V_{u d}\right|^{2} \mathrm{~N}_{c}\left(\mathrm{~N}_{c}^{2}-1\right)}{\left(2 \mathrm{u} \cdot \mathrm{d}-\mathrm{m}_{W}^{2}\right)^{2}} \frac{\mathrm{m}_{t}^{2}(\mathrm{~g} \cdot \mathrm{d})^{2}}{(\mathbf{t} \cdot \mathrm{g})^{2}}\left|\frac{\mathrm{u} \cdot \mathbf{b}}{\mathbf{t} \cdot \mathrm{d}}\right| .
$$

In pp colllision at the Tevatron with ${ }^{\mathrm{P}} \overline{\mathrm{s}} / 2.0 \mathrm{TeV}$, the antiproton beam supplies the d-type quark $98 \%$ of the time at $\mathrm{W}^{*}$ production. At $\mathrm{W}$-gluon fusion process, 
d-type quark is either contained in one of the beams or in the spectator |et. In fact, spectator let is the d quark $77 \%$ of the time [12]. The summary of the fractional cross section is shown in Table 1.3 for $\mathrm{W}^{*}$ process and Table 1.4 for Wg-fusion process. Figure 1.7 shows spin configuration of top decay products.

\begin{tabular}{|c|c|c|}
\hline $\mathrm{p}$ & $\overline{\mathrm{p}}$ & Fraction \\
\hline $\mathrm{u}$ & $\mathrm{d}$ & $98 \%$ \\
$\overline{\mathrm{d}}$ & $\mathrm{u}$ & $2 \%$ \\
\hline
\end{tabular}

Table 1.3. The fractional cross section for single top production in the $\mathrm{W}^{*}$ channel at the Tevatron with center of mass energy ${ }^{\mathrm{P}} \mathrm{s} / 2.0 \mathrm{TeV}$

\begin{tabular}{|c|c|c|}
\hline $\mathrm{p}$ & $\overline{\mathrm{p}}$ & Fraction \\
\hline $\mathrm{u}$ & $\mathrm{g}$ & $74 \%$ \\
$\mathrm{~g}$ & $\mathrm{u}$ & $3 \%$ \\
$\mathrm{~g}$ & $\mathrm{~d}$ & $20 \%$ \\
$\overline{\mathrm{d}}$ & $\mathrm{g}$ & $3 \%$ \\
\hline
\end{tabular}

Table 1.4. The fractional cross section for single top production in the $\mathrm{Wg}$-fusion channel at the Tevatron with center of mass energy ${ }^{\mathrm{p}} \overline{\mathrm{s}} / 2.0 \mathrm{TeV}$

In the limit of a massless b quark, b-quark decayed left-handedly . Also, W decay configuration is shown in Figure 1.8. The massless neutrio is left-handedly decayed, and then lepton is decayed backward in the left-handed W decay and goes to the opposite direction in the right-handed W decay to conserve angular momentum. Therefore, decayed lepton momentum is correlated to the direction of top spin(or d-type quark). The decay angular distributions are linear of decay angles (Fig. 1.9) with top spin vector in top quark rest frame [12].

$$
\frac{1}{\Gamma} \frac{\mathrm{dI}^{5}}{\mathrm{~d}\left(\cos \theta_{i}\right)} / \frac{1}{2}\left(1+\alpha_{i} \cos \theta_{i}\right) \text {. }
$$

where $\theta_{i}$ is the decay angle of the ith decay product (lepton, neutrino or b-quark) and the degree of correlation between decay product and the spin is represnented as $\alpha_{i}$. So, the these angle distribution are shown in 1.10. By using this top polarization, we test anomalous Wtb coupling and for this analysis, generate Monte Carlo with changing $V$-A to $V+\mathbf{A}$ coupling at the production W's vertices (called SM single top sample is $V$-A(LLLL), and non-SM(exotic) samle is $V+\mathbf{A}$ (RRLL); details in chapter 7). The fitted values are $\sigma / 1.43 \mathrm{pb}$ in $V$-A(LLLL) and $\sigma / 0.0$ pb in $V+\mathbf{A}(\mathrm{RRLL})$. Finally, the measured fraction of $V+\mathbf{A}$ coupling is $0 \pm 28 \%$. 

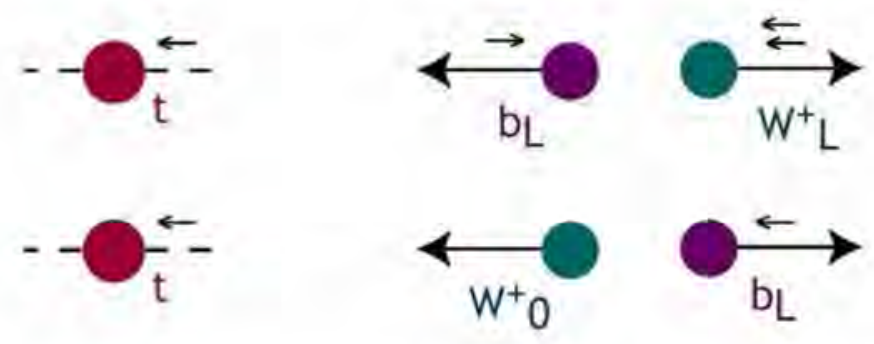

Figure 1.7. Allowed spin configuration for the top decay products in the limit of a massless b quark and each cases are left-handed(negative helicity) and longitudinal(zero helicity) W boson; small arrows represent each spin of top and bottom quarks and $\mathrm{W}$ boson.

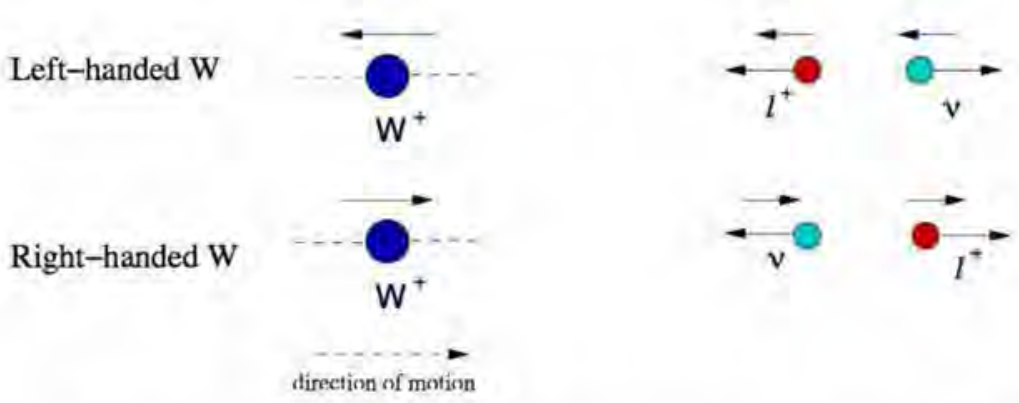

Figure 1.8. $\mathrm{W}^{+}$decay for left-handed and right-handed helicity. The charged lepton is decayed backward in the left-handed case and forward in the righthanded case due to angular momentum conservation. 


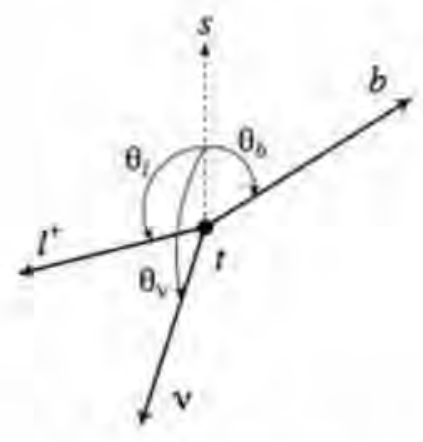

Figure 1.9. The decay angles of lepton, neutrino and $\mathbf{b}$ quark in the top quark rest frame. The vector $\mathrm{s}$ is the direction of top quark spin.

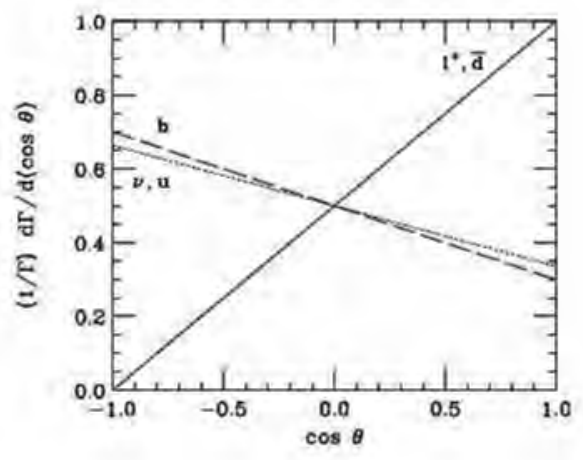

Figure 1.10. Angular correlations between the decayed particles and top quark spin vector in the decay of a spin up top quark. 


\section{Chapter 2}

\section{Experimental Apparatus}

\subsection{Accelerator}

At Tevatron, proton and anti-proton collisions started at the center of mass energy ${ }^{\mathrm{P}} \overline{\mathrm{s}} / 1.8 \mathrm{TeV}$ from 1985. The collected data from 1988 to 1996 (Run I) was $130 \mathrm{pb}^{-1}$ and instantaneous luminosity was $10^{31} \mathrm{~cm}^{-2} \mathrm{sec}^{-1}$. During this period, there was the discovery of top quark in 1995. From 1996, the upgrade of the accelerator was performed to increase the center of mass energy and the instantaneous luminosity. From 2001, Run II phase started at ${ }^{\mathrm{P}} \overline{\mathrm{s}} / 1.96 \mathrm{TeV}$ and instantaneous luminosity is about $10^{32} \mathrm{~cm}^{-2} \mathrm{sec}^{-1}$. Comparing to the Run I, the cross section of $\mathrm{t} \overline{\mathrm{t}}$ increased by $30 \sim 40 \%$ in Run II.

The accelerator is separated into several steps that is preparing the proton source. producing anti-proton source, the acceleration of both protons and antiprotons to the center of mass energy $1.96 \mathrm{TeV}$, and the collisions at CDF and D0.

The schematic view of the accelerator is shown in Figure 2.1.

Proton Source

The Cocksoft-Walton electrostatic Preaccelerator containing hydrogen atoms produces $\mathrm{H}^{-}$-ions(hydrogen consists of a proton and an electron). $\mathrm{H}^{-}$-ions are subsequently accelerated to an energy of $750 \mathrm{keV}$ by the difference of the electrostatic potentail and passed through a transfer line of focusing magnets to the Linear Accelerator(Linac) where the linear RF acceleration to an energy of 400 $\mathrm{MeV}$ occurs. After that, the electron in a $\mathrm{H}^{-}$-ion is stipped passing through a carbon foil leaving a proton and the separated protons are accelerated from 400 $\mathrm{MeV}$ to $8 \mathrm{GeV}$ in the Booster.

Main In|ector

The protons at $8 \mathrm{GeV}$ are inlected into the Main In|ector, which has a sheared over shape and has two fold symmetry, and are accelerated to $120 \mathrm{GeV}$ for fixed target operation or $150 \mathrm{GeV}$ for in|ection into the Tevatron. 


\section{FERMILAB'S ACCELERATOR CHAIN}

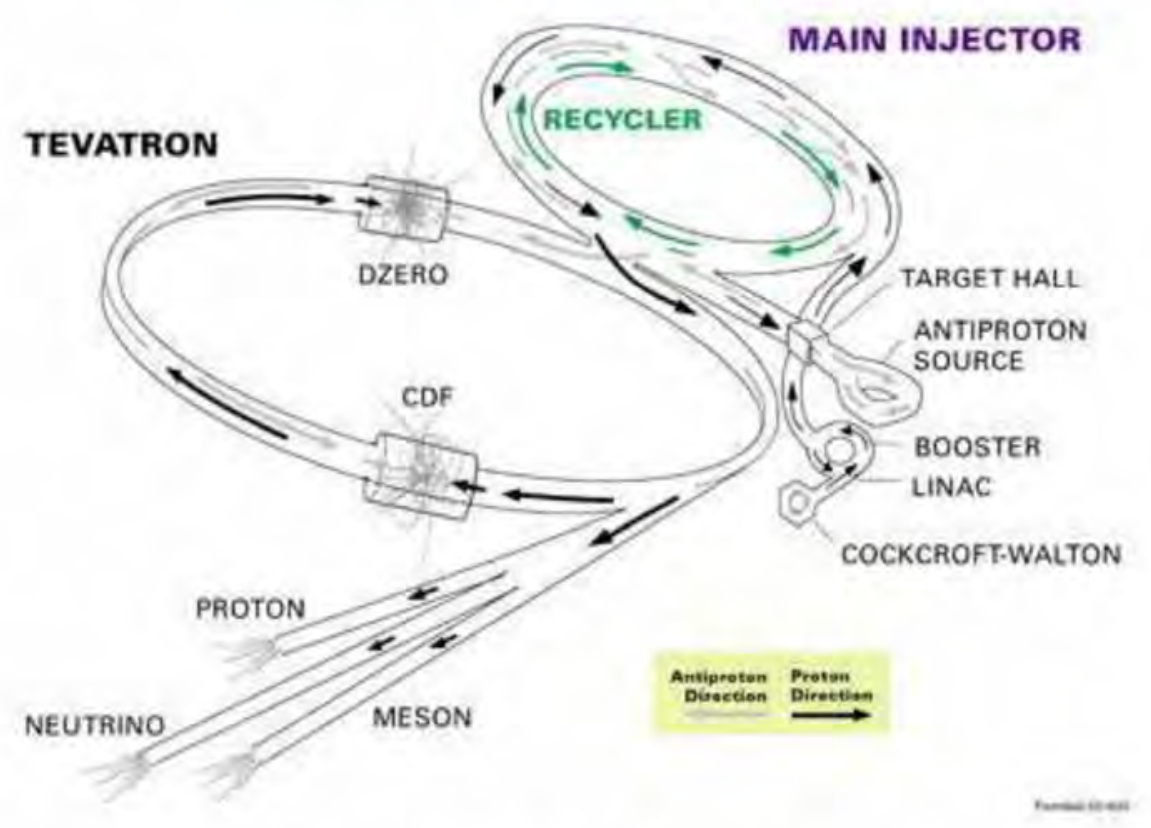

Figure 2.1. Schematic overview of the Fermilab accelerator chain. 


\section{Anti-proton Source}

A beam of $120 \mathrm{GeV}$ protons from Main In/ector is smashed on to a stack of nickel targets and copper cooling disks. The antiprotons coming out of the target at many different angles are focused into a beam line with a Lithium lens and then separated from many different particles through a pulsed magnet. A very large spread in energy of these antiprotons can change into the narrow energy spread by Debuncher accelerator. Additionally, the Debuncher maintains a stable antiproton beam at the energy of $8 \mathrm{GeV}$. Stochastic cooling systems achieves the beam stabilization by removing a wide range of energies, positions and angles of the antiprotons. The antiprotons remain in the Debuncher for about 2 sec until the next bunch of protons come and are sent to the Accumulator where the antiprotons are stacked at an energy of $8 \mathrm{GeV}$ and further cooled down. When the antiproton in the Accumulator reaches the maximum optimal capacity, the bunches are trasferred back to the Main In|ector and in|ected into the Recycler. Recycler

The Recycler is a fixed $8 \mathrm{GeV}$ kinetic energy storage ring and is located in the Main In/ector tumnel directly above the Main In/ector beamline. The Recycler sing provides long-term stash for antiprotons by acting as a high reliability postAccumulator and receptacle for recycled antiprotons from previous store. The further cooling of antiproton source occured using stochastic cooling and electron cooling in the Recycler.

Tevatron

Finally, the protons and antiprotons are sent to the Tevatron, which is a circular synchrotron with a diameter of about $2 \mathrm{~km}$. The 36 proton and 36 antiproton bunches are accelerated to the center of mass energy ${ }^{\mathrm{P}} \overline{\mathrm{s}} / 1.96 \mathrm{TeV}$. The collisions of focused proton and antiproton beam occure at CDF and B0,

\subsection{Detector: Upgrade of RunII Detector}

The CDF II detector, upgraded CDF detector from Run I in 1992 - 1995 period, is an azimuthal and forward-backward symmetric apparatus designed to study pp collisions at the Tevatron.

Figure 2.2 shows the detector.

\section{Tracking System}

The Tracking system consists of a silicon micro-strip detector and an open-cell wire drift chamber that surrounds the silicon detector. When a charged particle passed through the tracking system it ionizes the material/gas around it. A dedicated tracking algorithm searches for a continuous pattern of localized charge depositions (hits) and reconstructs the path of the charged particle, called the 


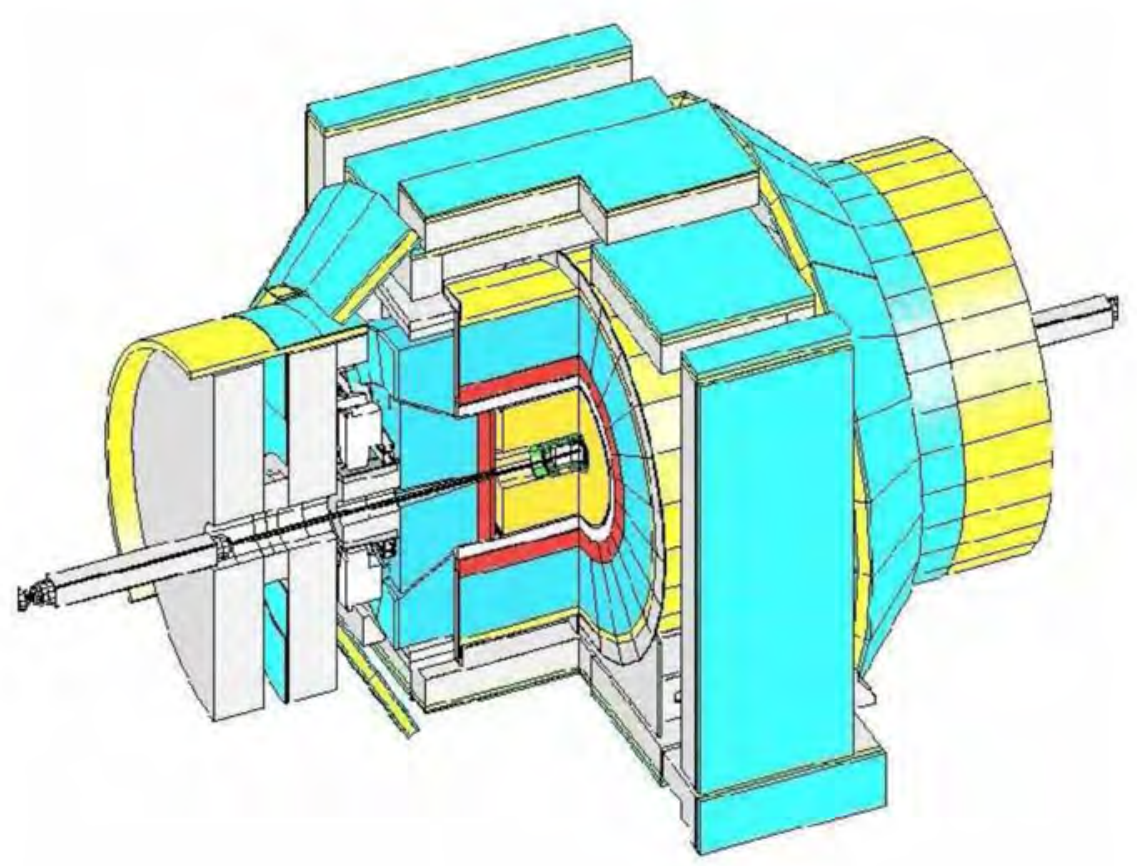

Figure 2.2. The CDF Run II Detector

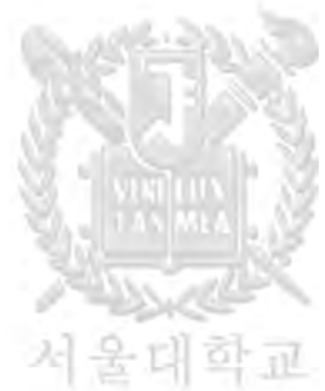

Copyright(c)2002 by Seoul National University Library.

All rights reserved.(http://library.snu.ac.kr) 
particle's 'track'. The longitudinal view of the CDF II tracking system is shown in Figure 2.3

\section{CDF Tracking Volume}

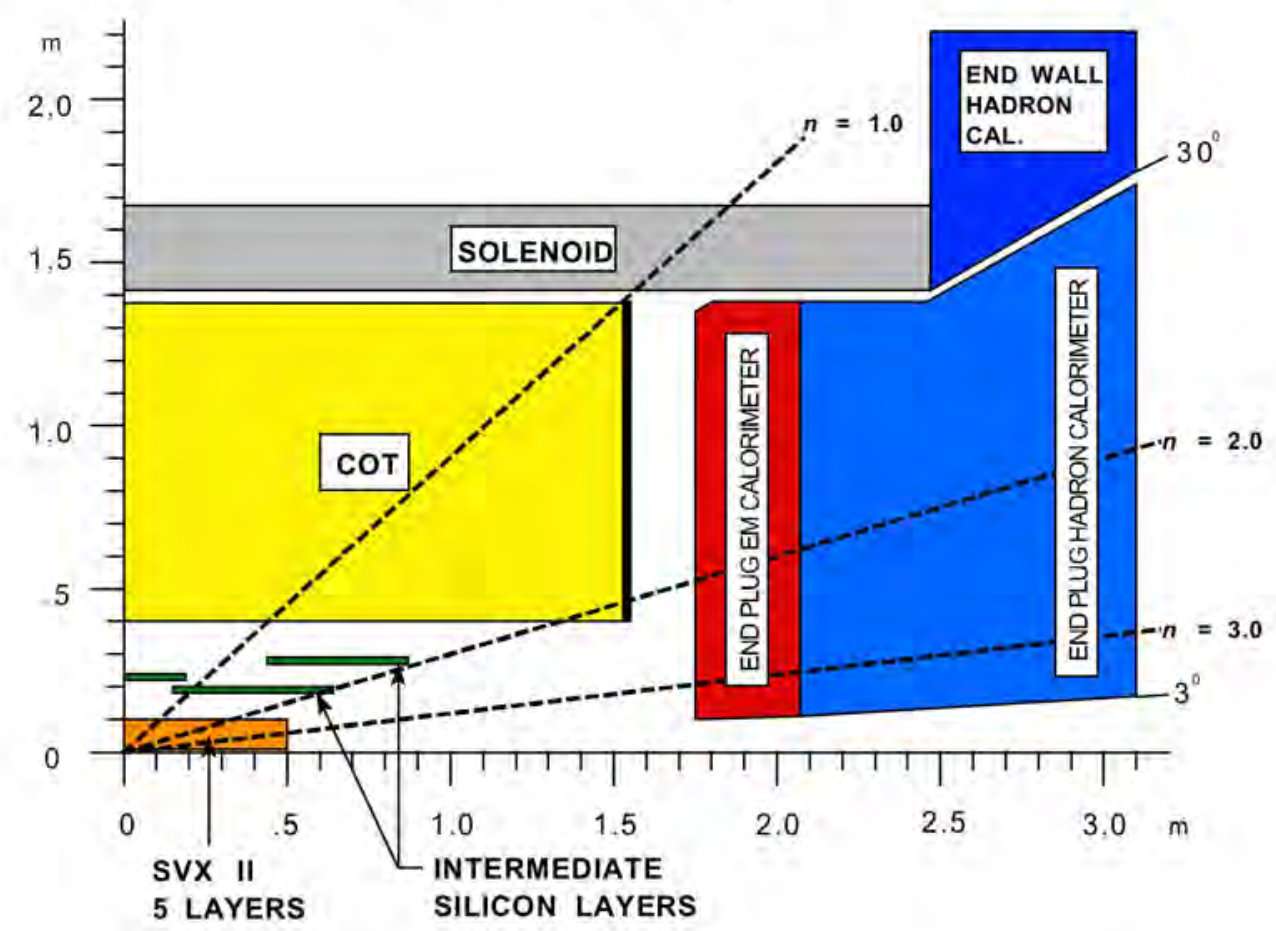

Figure 2.3. Longitudinal View of the CDF II Tracking System

The silicon micro-strip detector consists of three sub-detectors shown in Figure 2.4

The first is the Layer00 detector. This is a layer of radiation hard silicon wafers which is directly mounted around the beam pipe.

To detect secondary vertices from heavy flavor particle decays such as bottom and charm hadrons and to give an excellent tools for b-tagging, Silicon Vertex Detector(SVX) was added to CDF in 1992 for second sub-system. This detetor was replaced with $\mathrm{SVX} \mathrm{X}^{0}$ to control radiation damage using $\mathrm{AC}$-coupled detectors and a radiation hard readout chip. For increasing the number of $\mathrm{p}$ and $\overline{\mathrm{p}}$ bunches, $\mathrm{S} V \mathrm{X}^{0}$ detector was substituted for SVX II (Run II Silicon Vertex Detector) which is designed to permit good resolution in locating the z-position of secondary vertices and to enhance the 3 -D pattern recognition capability of the 


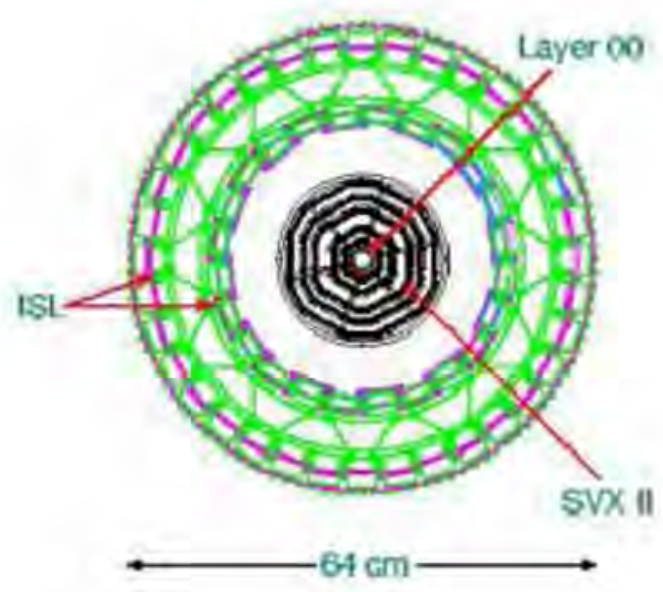

Figure 2.4. Silicon micro-strip detector consists of Layer 00, SVX II and ISL.

tracker. This detector has three barrels(Fig. 2.5), each $29 \mathrm{~cm}$ long. There are 12 wedges in $\phi$, each with five layers of silicon; three have $0^{\circ}-90^{\circ}$ stereo and two have $1.2^{\circ}$ small-angle stereo. Some mechanical properties are given in Table 2.1. For each barrel, the silicon ladders are mounted between two precision-machined beryllium bulkheads(Fig.2.6) which also carry the water cooling channels for the readout electronics.

For $|\eta|>1$, SVX II can only perform 2D tracking and impact parameter resolution for such tracks will be too poor to enable efficient $b$ tagging. These problems are addresed by the Intermediate Silicon Layers(ISL). In the central region, a single layer of silicon is placed at a radius of $22 \mathrm{~cm}$ and the region, $1.0 \leq|\eta| \leq 2.0$, two layers of silicon are placed at radii of $20 \mathrm{~cm}$ and $28 \mathrm{~cm}$. Thus, ISL extends tracking, lepton indentification, and b-tagging capabilities over the full region $|\eta| \leq 2.0$

The new open-cell drift chamber, referred to as Central Outer Tracker (COT), is located directly outside of the silicon tracking detectors in the radial direction. The chamber consists of eight-layers (SL) of $310 \mathrm{~cm}$ length cells and radii between 40 and $132 \mathrm{~cm}$ from the beam axis. The COT is designed to operate with a maximum drift of $100 \mathrm{nsec}$ by using a gas mixture containing 50.35.15 Ar-Et-CF . $_{\text {. }}$ drift velocity is $\sim 100 \mu \mathrm{m} / \mathrm{ns}$ which implies a maximum drift distance of $\sim 1 \mathrm{~cm}$. In Run II, to improve the robustness of the COT superlayers each consist of 12 sense wires, |ust like the axial layers. At CTC(Run I), The electrostatics of a cell is shaped by field wires whose potential varies to account for the tapered shape of the cell. In the COT(Run II), the field wires are replaced by as cathode "field 


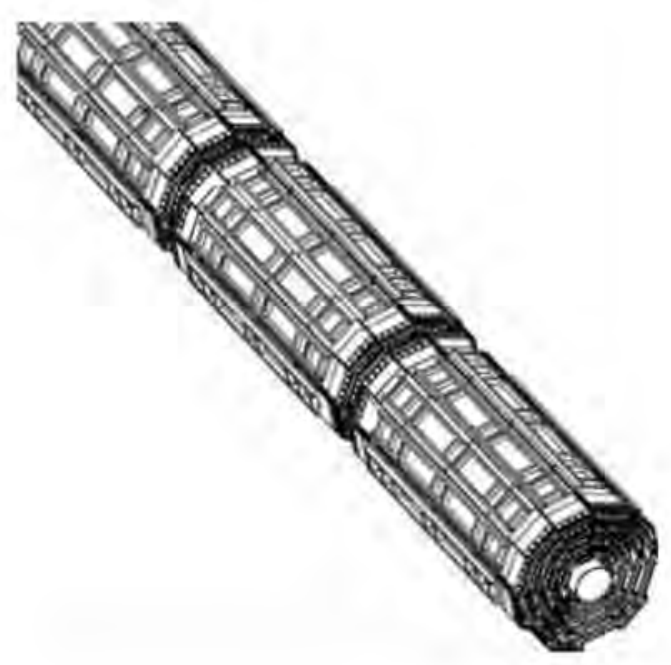

Figure 2.5. The SVX II 3 barrels

\begin{tabular}{|c|c|c|c|c|c|}
\hline Property & Layer0 & Layer1 & Layer2 & Layer3 & Layer4 \\
\hline number of $\phi$ strips & 256 & 384 & 640 & 768 & 896 \\
\hline number of $Z$ strips & 256 & 576 & 640 & 512 & 896 \\
\hline number of $\phi$ chips & 2 & 3 & 5 & 6 & 7 \\
\hline number of $Z$ chips & 2 & 3 & 5 & 4 & 7 \\
\hline stereo angle & $90^{\circ}$ & $90^{\circ}$ & $+1.2^{\circ}$ & $90^{\circ}$ & $-1.2^{\circ}$ \\
\hline$\phi$ strip pitch $(\mu \mathrm{m})$ & 60 & 62 & 60 & 60 & 65 \\
\hline$Z$ strip pitch $(\mu \mathrm{m})$ & 141 & 125.5 & 60 & 141 & 65 \\
\hline total width $(\mathrm{mm})$ & 17.140 & 25.594 & 40.300 & 47.860 & 60.170 \\
\hline total length $(\mathrm{mm})$ & 74.3 & 74.3 & 74.3 & 74.3 & 74.3 \\
\hline active width $(\mathrm{mm})$ & 15.300 & 23.746 & 38.340 & 46.020 & 58.175 \\
\hline active length $(\mathrm{mm})$ & 72.43 & 72.43 & 72.38 & 72.43 & 72.38 \\
\hline number of detectors & 144 & 144 & 144 & 144 & 144 \\
\hline
\end{tabular}

Table 2.1. Silicon detector mechanical dimensions 


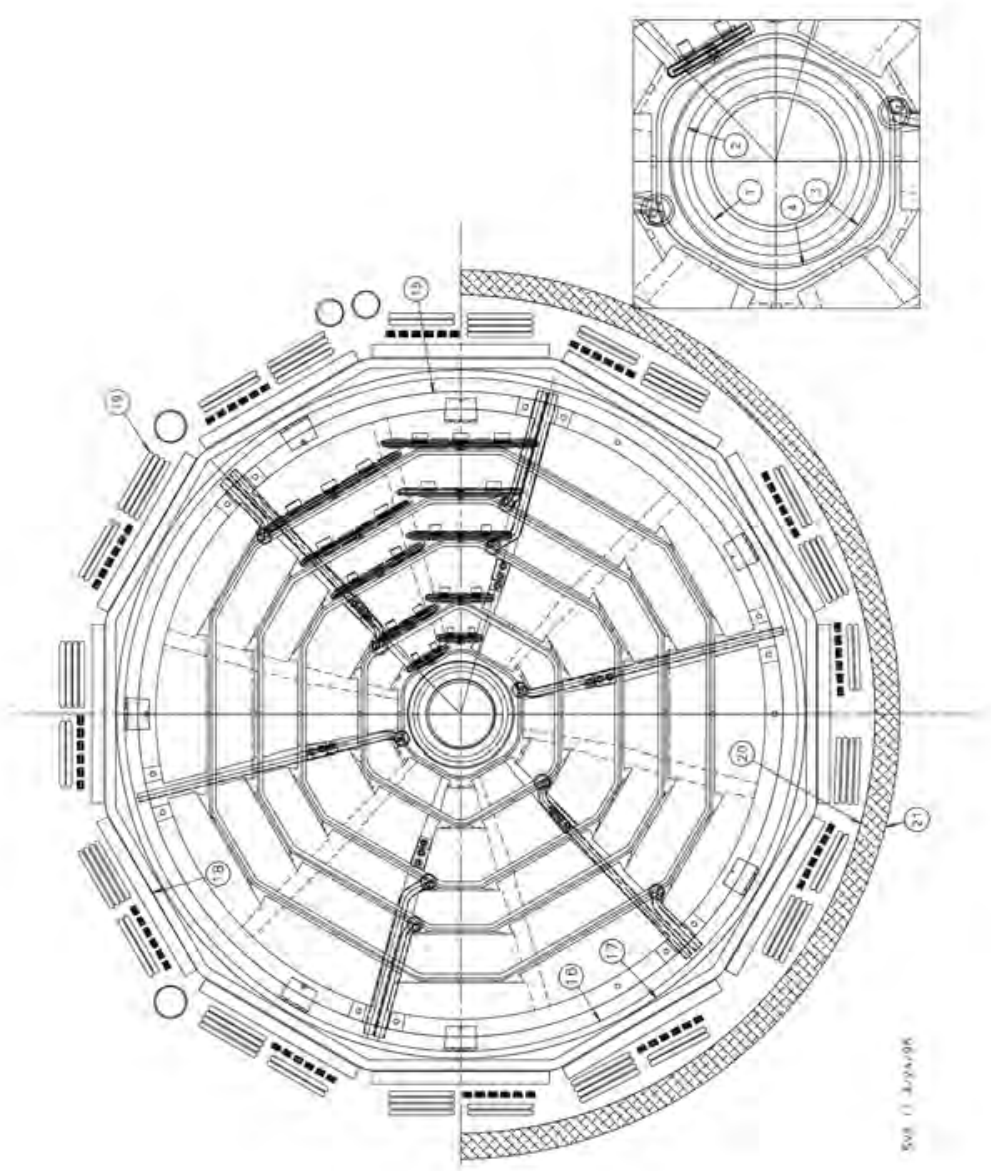

Figure 2.6. The SVX II bulkhead design 
panel" which is gold on a 0.25 mil thick Mylar sheet. The ends of each cell are closed, both mechanically and electrostatically, by Mylar strips with field-shaping wires attached. these are referred to as "shaper pannels". Between the field panels, sense wires alternate with potential wires in a plane. The 1.6 mil gold-plated tungsten is used for both wires. Figure 2.7 illustrates the cell geometry using SL2 as an example.

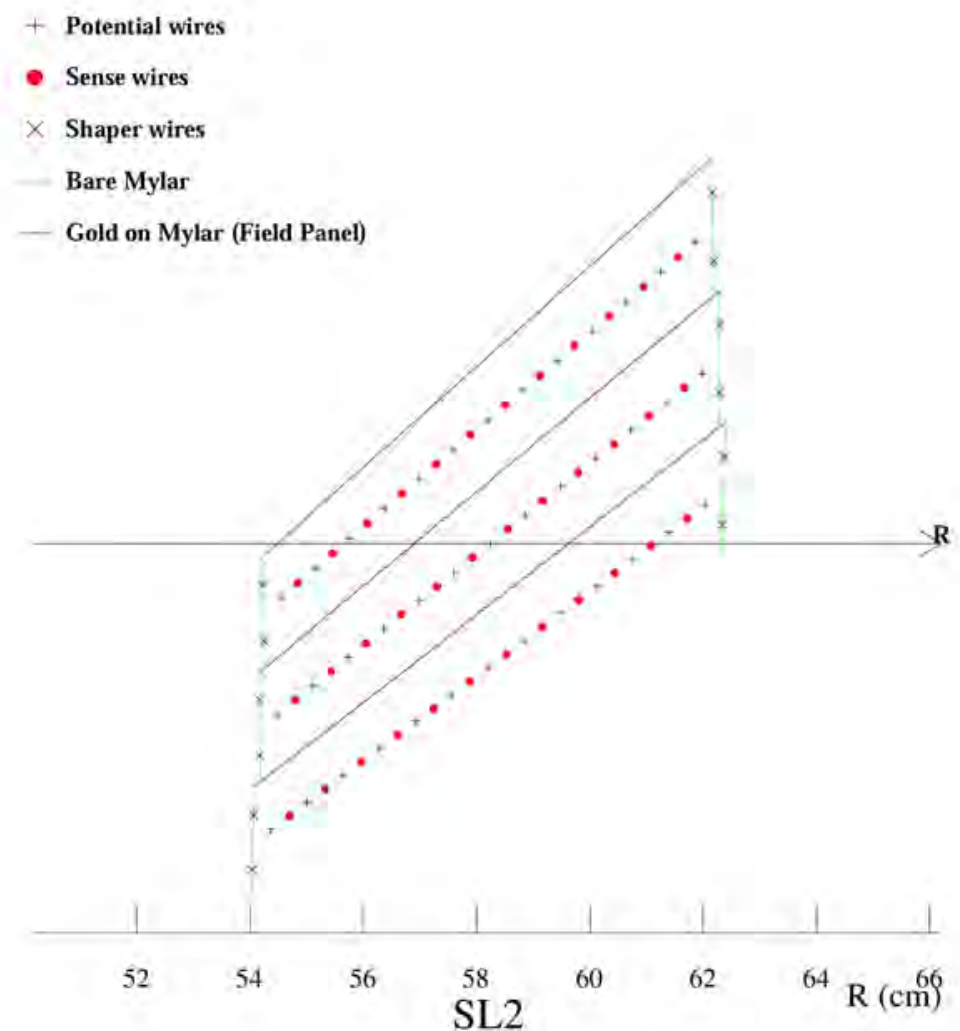

Figure 2.7. Nominal cell layout for SL2.

The charge and momentum of a particle-track are determined by measuring the curvature of the track in the magnetic field. The solenoid produces a $1.4 \mathrm{~T}$ magnetic field inside the tracking volume that is uniform to $0.1 \%$ in the acceptance region. The transverse momentum of a reconstructed track is determined from $\mathrm{P}_{T} / \mathrm{Bqr}$, where $\mathrm{B}$ is the strength of the magnetic field, $\mathrm{q}$ is the charge of the 


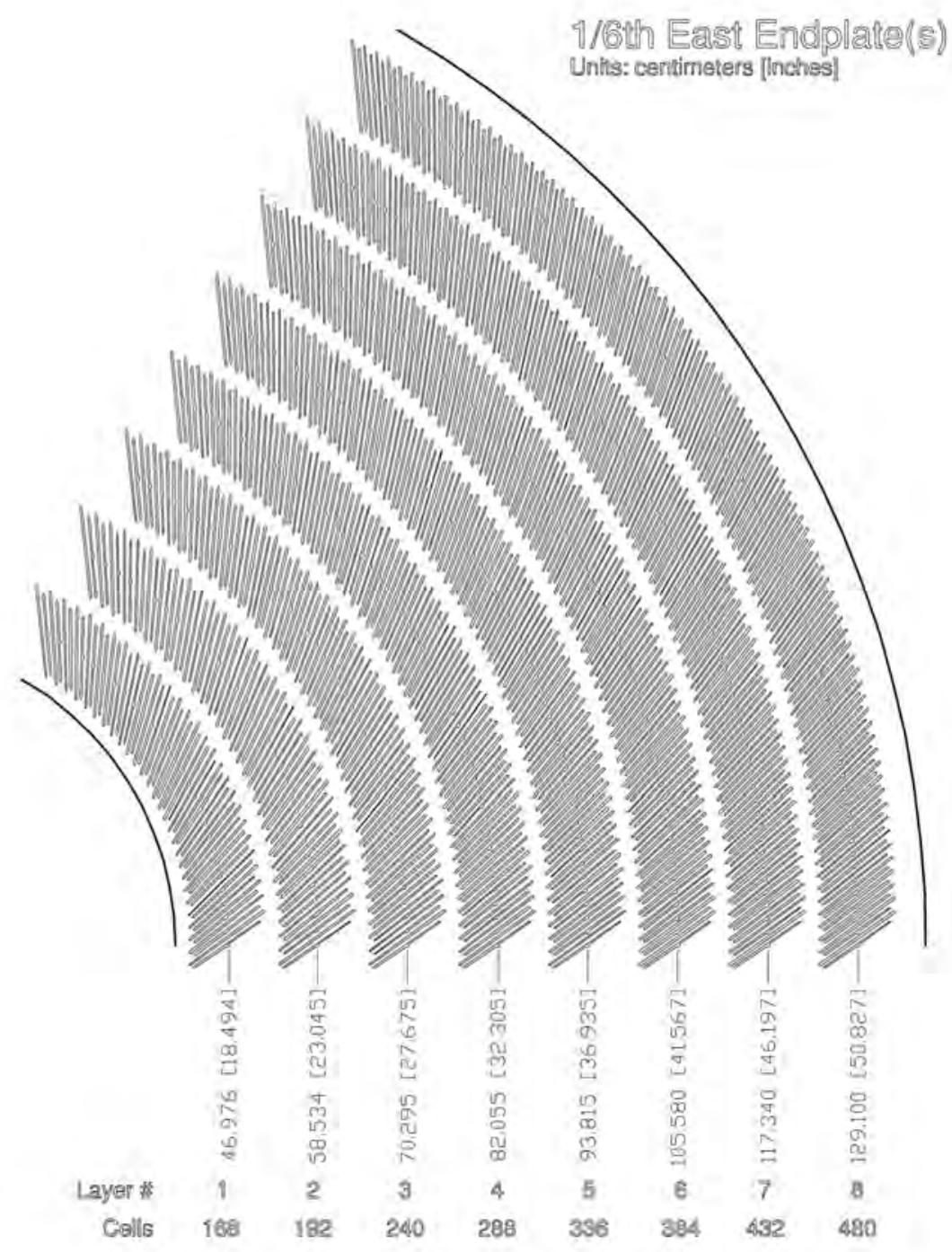

Figure 2.8. East endplate slots. Sense and field planes are at the clock-wise edge of each slot. 


\begin{tabular}{|c|c|}
\hline Number of Layers & 96 \\
\hline Number of Superlayers & 8 \\
\hline Stereo Angle & $+3.0-3.0+3.0-3.0^{\circ}$ \\
\hline Cells/Layer & 168192240288336384432480 \\
\hline Sense wires/Cell & 1212121212121212 \\
\hline Radins at Center of SL & $4658708294106119131 \mathrm{~cm}$ \\
\hline Sense wire Spacing & $0.3^{\mathbf{\Phi}}(7.62 \mathrm{~mm})$ in plane of wires \\
\hline Wire Diamter & 1.6 mil gold plated Tungsten \\
\hline Wire tension & $135 \mathrm{~g}$ \\
\hline Tilt Angle & $35^{\circ}$ \\
\hline Length of Active Region & $310 \mathrm{~cm}$ \\
\hline Total number of Wires & 63000 \\
\hline Endplate Load & $\sim 40$ metric tons \\
\hline Drift Field & $2.5-3 \mathrm{kV} / \mathrm{cm}$ (depending on gas) \\
\hline
\end{tabular}

Table 2.2. COT Mechanical Summary

particle and $\mathbf{r}$ is the measured radius of curvature. The momentum resolution of the COT is given by $\sigma_{P t} / \mathrm{P}_{T}^{2} / 1.7 \times 10^{-3}|\mathrm{GeV} / \mathbf{c}|^{-1}$

\section{Calorimeter System}

The CDF calorimeter is a scintillator sampling system measuring the energy of particles produced in the $p \bar{p}$ collision. The calorimeter is located behind the tracking volume and consists of layers of active (scintillator) material sandwiched between sheets of absorbing material such as lead and steel. As particles pass through the calorimeter, they interact with the layers. The photons produced in the scintillator layers and directly go to the photomultiplier by the plexiglass guides. Figure 2.9 shows the calorimeters in both central and plug region.

The central region which extends to a pseudorapidity $(|\eta|)$ of about 1 is spanned by CEM (lead ( $1 / 8 \mathrm{inch})$-scintillator( $5 \mathrm{~mm}$ polystyrene) Electro-Magnetic calorimeter) followed by CHA (iron ( $2.5 \mathrm{~mm}$ ) - scintillator (1 cm PMMA) hadron calorimeter). Both calorimeters have the basic stucture of $15^{\circ}$ wedges which are subdivided in 10 pro/ective towers. EM calorimeter has provided effective indentification of electrons and photons. Hadrons(strongly interactina particles), on the other hand, shower over longer distances in the calorimeter and deposit the most significant fraction of their associated energy in the hadronic section of the calorimeter.

CEM light-output is $\sim 100$ photoelectrons per $\mathrm{GeV}$ per PMT and design energy resolution $\sigma(\mathrm{E}) / \mathrm{E}$ for electrons is $13.5 \% / \overline{\mathrm{E} \sin 0}$. 
CHA has good resolution(33\%/ $\mathrm{p} \overline{\mathrm{E} \sin \theta}$ with a $4 \%$ constant term) for $50 \mathrm{GeV}$ pions but there's the limitted thickness due to financial and structural limitations. The WHA(Wall Hadron Calorimeter) is in $|\eta|$-range $\sim 1.1$ complementing CHA. This detector is also an iron (5 cm)-scintillator(1 cm PMMA) but the sampling fraction is smaller than for the CHA because higher forward energies require more absorber and its resolution is worse.

The forward plug calorimeter has been upgraded for Run II. . The original gas calorimeter was replaced with scintillator plate calorimetry using scintillator tiles readout by wavelength shifting fibers embedded in the scintillator. Both the new plug electromagnetic calorimeter (PEM) and the new plug hadronic calorimeter (PHA) use the same polystyrene based scintillator and photomultiplier tubes used in the CEM. The PEM contains 23 layers of $0.45 \mathrm{~cm}$ of lead interleaved with 4.0 $\mathrm{mm}$ of scintillator while the PHA is made of 23 layers of $2.5 \mathrm{~cm}$ steel interleaved with $1.0 \mathrm{~cm}$ of scintillator. These calorimeters span the range $1.1 \leq|\eta| \leq 3.64$. Detector performance is $16 \% / \mathrm{P} \overline{\mathrm{E}}+1 \%$ for the PEM with 5 p.e./minimally-ionizing particle(mip)/tile corresponding to a total light output of 400 p.e./GeV. For the PHA, is is $70 \% / \overline{\mathrm{E}}+4 \%$ with 5 p.e./mip/tile correspoding to a total light output of 40 p.e./GeV Figure 2.10 shows the cross-section of end plug calorimeter.

\section{Muon System}

The muon detectors are located behind the calorimeter modules. Muons typically pass through the calorimeter modules leaving only a small fraction of their energy in the calorimeter. The muon system consists of four independent system of proportional wire chambers, filled with a 50.50 of argon and ethane, and scintillators.The Central Muon detector(CMU) and the Central Muon Upgrade(CMP) both cover $0<|\eta|<0.6$; the Central Muon extension (CMX) which covers 0.6 $<|\eta|<1.0$; and the Barrel Muon Upgrade Detector (BMU) which covers 1,0 $<|\eta|<1.5$.

The CMU consists of four layers of drift chamber and can detect muons with a transverse momentum $\mathrm{p}_{T} \geq 1.4 \mathrm{GeV} / \mathrm{c}$ The CMP consists of four layers of wire drift chamber of identical design to the CMU, but they are staggered by half cell per layer. The chambers are located behind $60 \mathrm{~cm}$ of steel and form a rectangular box around the central detector. The CMP is mainly used in combination with the CMU detector to improve the purity in muon identification and the combined system is called CMUP. The Central Scintillator Upgrade (CSP) is located on top or the outermost layer of the CMP. The CSP consists of a single layer of scintillator plates to provide additional timing information.

The CMX is a conial arrangement of drift chambers similar to those of the CMP. Different to the CMU and CMP, this detector consists of eight layers of rectangular chambers which are grouped in pairs to form four continuous layers. 


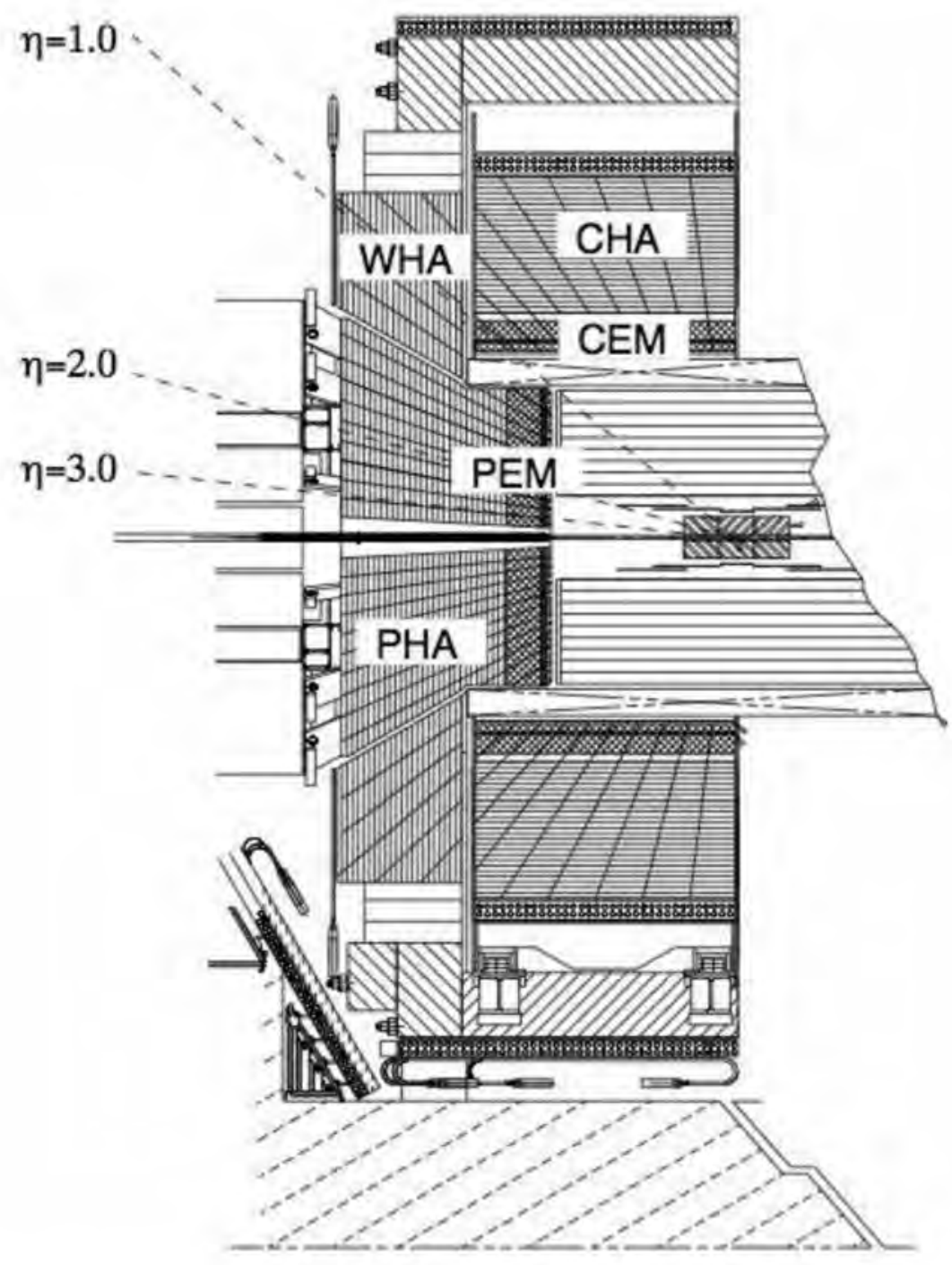

Figure 2.9. Calorimeter at CDF. CEM, CHA (central region $|\eta|<1.0$, plug region. PHA, PEM, WHA) 


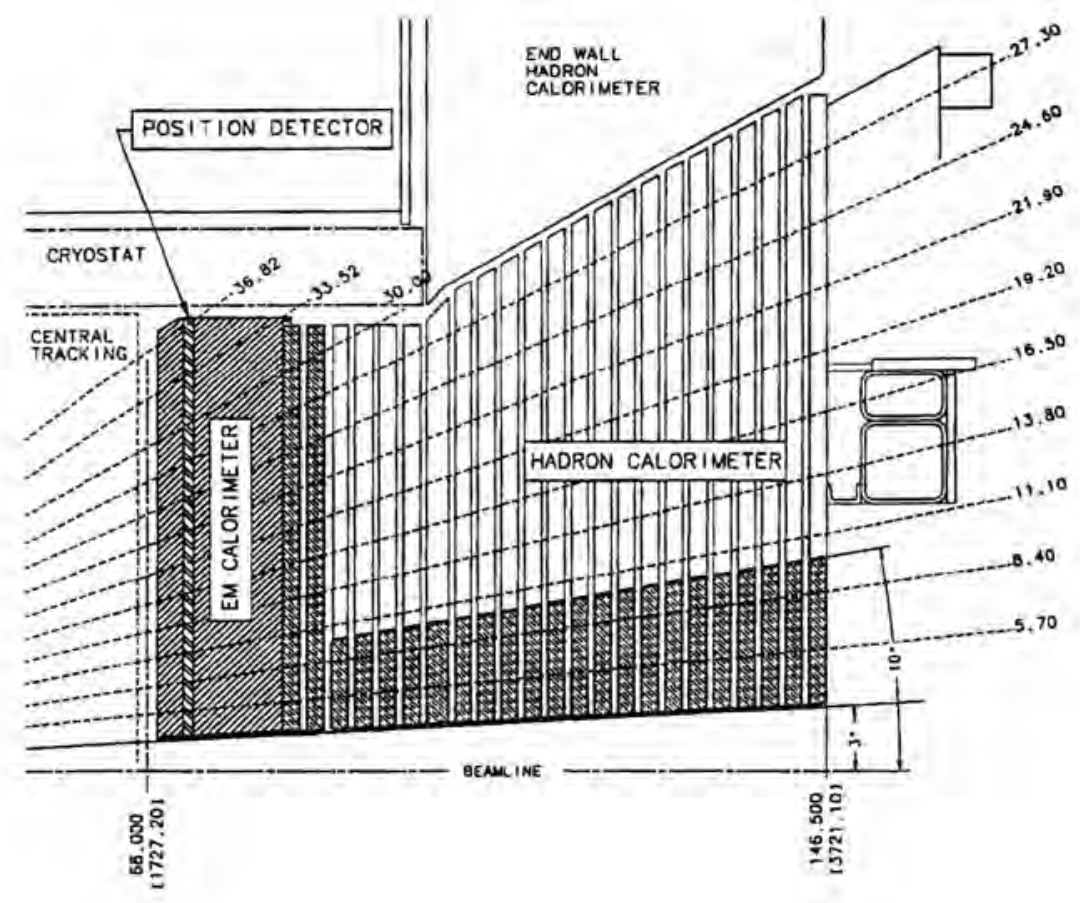

Figure 2.10. Cross section of upper part of new end plug calorimeter 
The Central Muon Extension (CSX) are mounted on both sides of the CMX system which is used in coincidence with the wire chambers to further improve the timing of the system and reduce the fake rate such as beam splashes.

The BMU and BSU(Barrel Scintillator Upgrade) provides momentum and timing measurement. Those detectors are installed on top of the forward toroid barrels. At $t-\phi$ plane, the geometrical coverage of the muon chamber is shown in 2.11 .

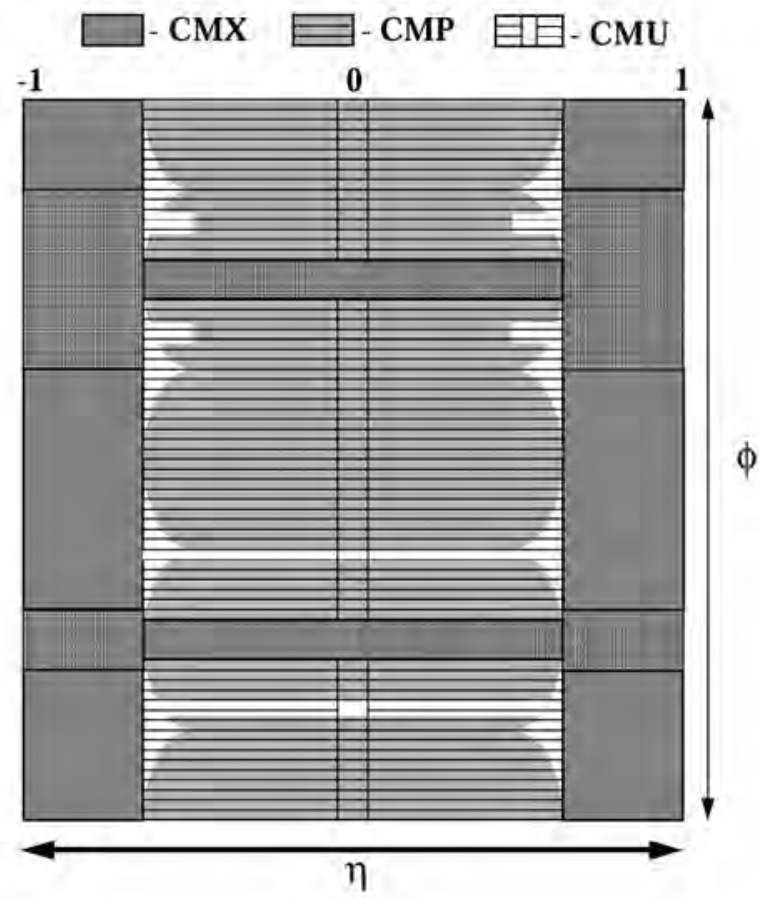

Figure 2.11. Geometrical Coverage of the Muon Chambers in $\eta-\phi$ plane

\subsection{Trigger and Data Acquisition}

The trigger is important system for accepting events because the collision rate is much higher than the rate at which data can be stored on tape. The interesting physices events need to be extracted from the large number of minimum bias events. The entire trigger system used in Run I is replaced for Run II because 
the accelerator and detector upgrade. The main reason for replacing the trigger electonics is the reduction of the accelerator bunch spacing from $3.5 \mu$ sec to 132 396 nsec. The CDF trigger system is composed of three level constituents which is Level 1, Level 2, Level 3.

The first level of the tigger(Level 1) has to be the fastest and uses custom hardware to select events based on information from the calorimeters, tracking chamber(COT) and muon detectors. All detector data are fed into a $6 \mu \mathrm{s}$ pipeline to provide time for processing required at Level-1. The eXtremly Fast Trigger(XFT) reconstructs tracks in the transverse plane of the COT. The XTRP then matches the tracks to electromagnetic calorimeter energy clusters or track segments in the muon systems. The acceptance rate of Level 1 is about $30 \mathrm{KHz}$. The Level 2 trigger used custom hardware to do a limited event reconstruction, Processing of Level 2 trigger starts after the event is written into one of the four Level 2 buffers on all front-end and trigger modules by a Level 1 accept. The buffer cannot be used for additional Level 1 accepts during analyzing data at Level 2. This trigger level get the information from the SVX and CES(central shower-max detector). The CES(shower maximum detector) designed to improve identification of electrons and photons. The electron and photon trigger hardware is upgraded for RUN II using the information from the central shower maximum detector(CES). This upgrade made $50 \%$ reduction of backgrounds for electron. The Silicon Vertex Tracker(SVT) enables to increase the physics reach using impact parameter informatin to detect secondary vertices. The acceptance rate of Level 2 is about $300-1000 \mathrm{~Hz}$.

The Level 3 trigger uses the full detector resolutions to fully reconstruct events in a processor farm. The developments aim for the reduction of event size to increase the Level 3 accept rate. After passing Level 3, the events are sent to the permanet storage at a rate of about $75-100 \mathrm{~Hz}$. The dataflow of trigger system is shown in Figure 2.12.

\subsection{Offline Data Reconstruction}

\section{Monitering and Finding of Beam Position}

The beamline calibration is one of works of the offline group. The position of the beamline is an important input for many physics analyses such as event reconstruction for missing Et. Fitting beam position is performed by using track information of SVX and COT.After fitting the beamline, the fitting results (beamx, beamy, slopex, slopey, etc.) are stored in Data Base by each run. The fitted results can be used to monitering of tracking detector performance. The tracking alignment can be checked from comparing beam position of SVX and COT.

We used two algorithns for fitting beamlines. The first algorithm is based on 


\section{Dataflow of CDF "Deadtimeless" Trigger and DAQ}

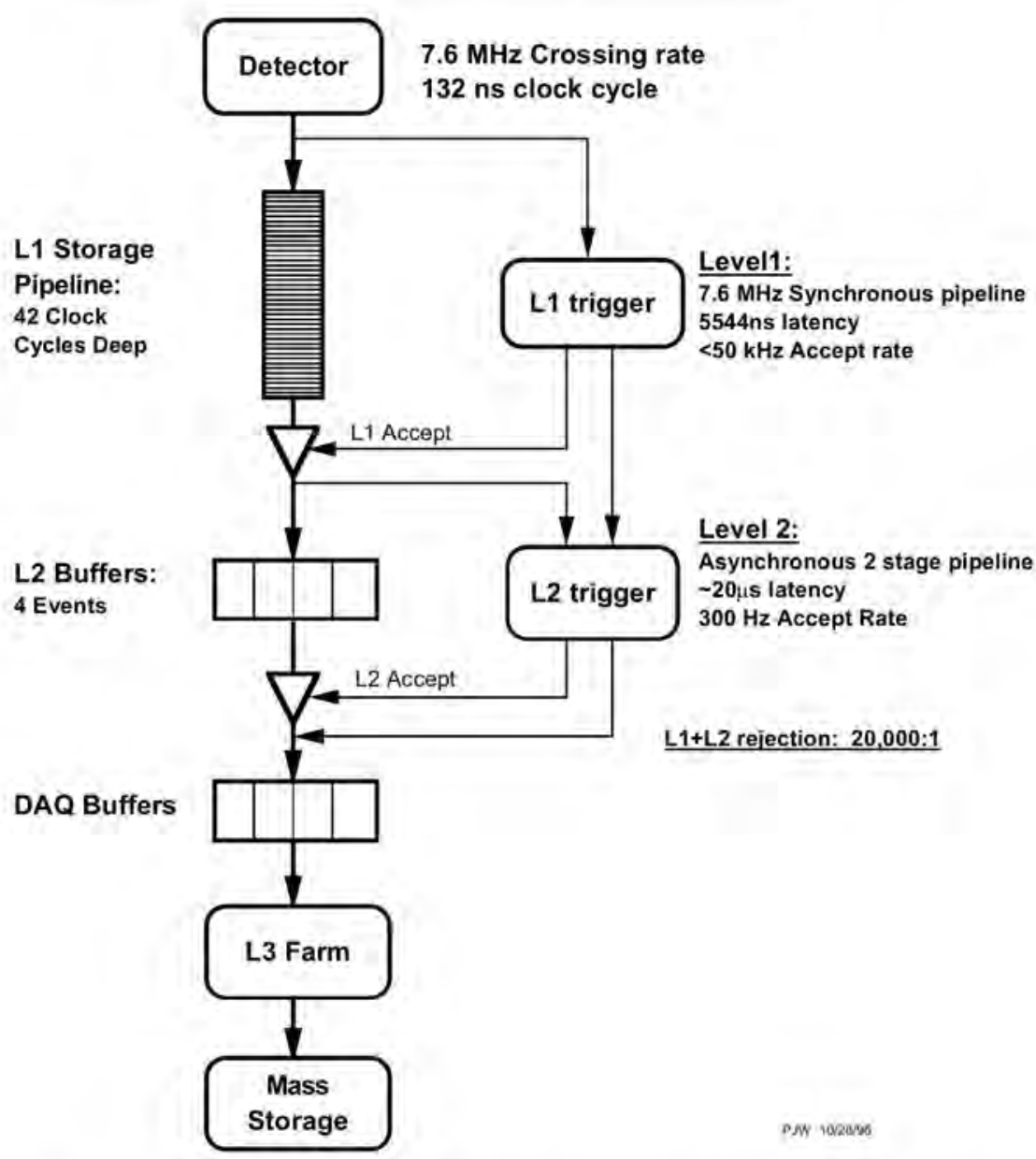

Figure 2.12. Fundamental block diagran of the CDF II data flow 
the reconstruction of the primary vertex for each event using tracks. The second algorithm is based directly on tracks using the correlation of the $d_{0}$ and the $\phi_{0}$ parameter of a track. The detailed description and fitted results for each run is in Appendix A. The explanation about the fitting algorithm is described in A.1 and the plots for beam position about svx and cot for each run are shown in A.2.

The $\mid \mathrm{ob}$ of fitting beam position is performed three times a day by cron $\mid \mathrm{ob}$. The cron |ob runs main script(autoValidate.pl) and check if the runs are already fitted runs in good-run list.

If all runs are valid within certain run range(processed runs in good run list), the information of beam about those runs is stored in Data Base. The values of $\mathrm{x}$-position and $\mathrm{y}$-position, slope in $\mathrm{xz}$ and $\mathrm{yz}$ plane and beam width and so on are stored in Data Base. 


\section{Chapter 3}

\section{Monte Carlo Simulation}

\subsection{Signal: s-channel, t-channel}

The single-top signal events are produced with MADEVENT and PYTHIA. MADEVENT is a tree-level generator which is powered by the matirx element generator MADGRAPH. The amplitudes and event for each process are generated by MADEVENT. Because MADEVENT is designed to produce events at parton level, it interfaces with PYTHIA for showering and hadronization of events. The PYTHIA is a multi-particle production generator such as hard and soft interactions, parton distributions, initial and final-state parton showers, multiple interactions, fragmentation and decay.

s-channel Single Top-Quark Production

In s-channel single-top production, NLO distributions are the same as the LO distributions multiplied only by a constant correction. ZTOP software package provides NLO s-channel and t-channel single top-quark production distribution within the geometrical acceptance of a given detector. The ZTOP software calculates, that at least one /et(apart from the $1^{\text {st }}$ b-quark /et coming from the top-quark decay) should be visible within the detectors acceptance in about $90 \%$ of all s-channel events. In most events, this $\mathrm{p}_{T}$-leading let is a bquark from the top decay. In MADEVENT, it is represented by a $2^{\text {nd }}$ b quark at parton level. Only in about $10 \%$ ZTOP expects a light quark let, which could come from hard initail or final-state gluon radiation and initail-state gluon splitting. Since the s-channel MADEVNET sample is LO and does therefore not include matrix elements with real gluon corrections modeled by the PYTHIA showering. 
t-channel Simulation Matching Procedure

The PYTHIA generates too soft and too far forward distributed $2^{\text {nd }} \mathbf{b}$ quarks. Because PYTHIA starts with the LO $2 \rightarrow 2$ diagram, that is with a b-quark PDF and then creates the initial state using backward evolution(DGLAP). In LO process, soft region of the transverse momentum is well modeled, but the hard region is underestimated. The $2 \rightarrow 3$ NLO processes are generated with the initial state gluon splitting into a $\bar{b}$ for hard region. Therefore, we need to match those two samples of $2 \rightarrow 2 \mathrm{LO}$ and $2 \rightarrow 3$ NLO. A |oint èvent sample is created by matching the $\mathrm{p}_{T}$ spectrum of the $2^{\text {nd }}$ b quarks in the matched $\mathbf{t}$-channel sample consists of $2 \rightarrow 2$ events for transverse momenta below a certain cutoff $K_{T}$ and of $2 \rightarrow 3$ events for transverse momenta above $\mathrm{K}_{T}$. The ratio $\mathrm{R}$ between the two processes are varied until the rate of events with a detectable $2^{\text {nd }} \mathbf{b}$ quark |et, that is $\mathrm{p}_{T}>$ $20 \mathrm{GeV} / \mathbf{c}$, of the oint t-channel sample are simulated using $2 \rightarrow 3$ sample. The Figure 3.1 shows the 2 nd $\mathrm{b}$-quark Pt distributions of LO and NLO events.

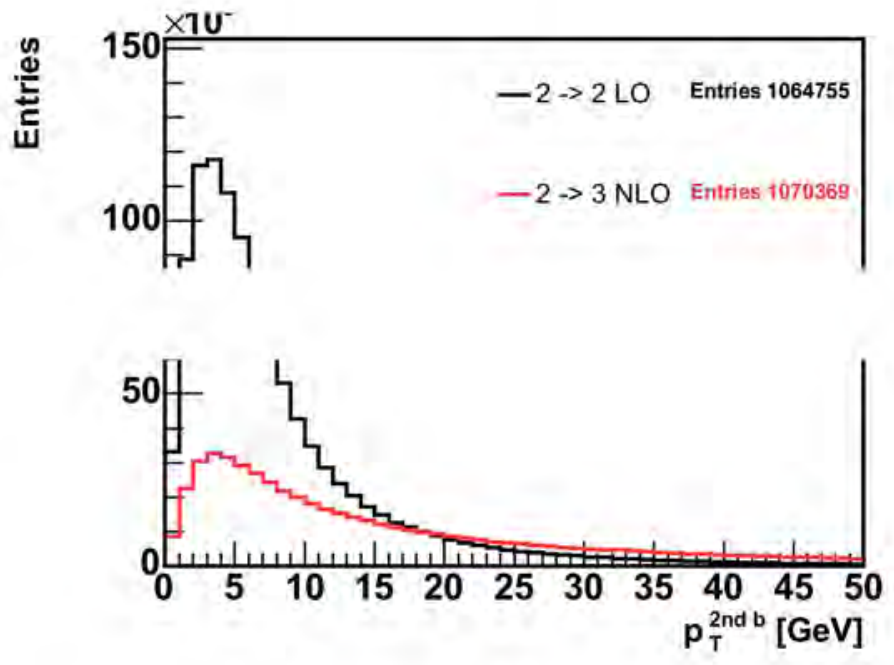

Figure 3.1. 2nd b-quark Pt distributions of LO and NLO 
The Figure 3.2 shows the $2 \mathrm{nd}$ b-quark Pt distributions of LO and NLO events in $\log$ scale.

Finally, obtained value of $K_{T}$ is $18 \mathrm{GeV}$ for single-top t-channel(stopm0) sample.

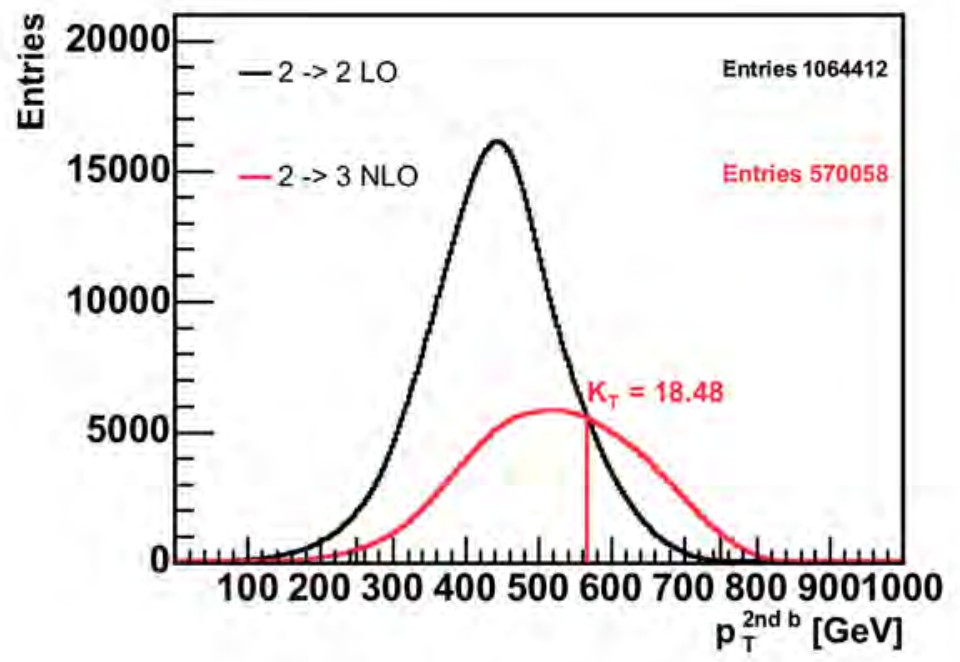

Figure 3.2. 2nd b-quark Pt distributions of LO and NLO in log scale with $\mathrm{K}_{T} / 18$ $\mathrm{GeV}$ 


\subsection{Backgrounds}

\subsubsection{Top-Antitop}

For the $t \bar{t}$ production, the events are generated with PYTHIA. The $t \bar{t}$ (top pair production) can be detected as single top events. There are two cases. One W decays leptonically and the other decays into two lets in the lepton plus lets and the other case is both of two bosons from top decay decay leptonically, but one lepton lost in detector.

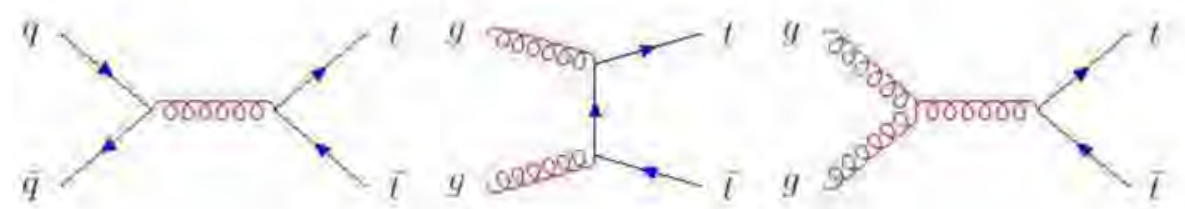

The simulated events (PYTHIA Monte Carlo sample) are used to calculate the expectation by normalizing the Monte Carlo to the theoretical cross section. The number of expected events is calculated as

$$
\mu / \sigma \cdot \epsilon_{e v t} \cdot \mathrm{L}_{i n t}
$$

where $\sigma$ is theoretically predicted cross section, $\epsilon_{e v t}$ is event detection efficiency, and $L_{i n t}$ is the integrated luminosity.

The event detection efficiency can be calculated by selecting simulated events.

$$
\epsilon_{\text {evt }} / \epsilon_{m c} \cdot \epsilon_{B R} \cdot \epsilon_{\text {corr }} \cdot \epsilon_{\text {trig }}
$$

where $\epsilon_{m c}$ is detection efficiency from MC, $\epsilon_{\text {trig }}$ is the trigger efficiency, $\epsilon_{c o r r}$ is the correction factor between data and simulation and $\epsilon_{B R}$ is the branching fraction.

\subsubsection{W + Heavy Flavor}

To estimate of $\mathrm{W}+$ Heavy Flavor processes $(\mathrm{W} \mathbf{b} \overline{\mathbf{b}} \mathrm{W} \mathbf{c} \bar{c}, \mathrm{Wc})$, the number of direct $\mathrm{W}+$ ets events, $\mathrm{N}_{W+\text { jets }}^{N_{\text {jet }}}$, are measured by using pre-tag data sample. The number of $\mathrm{N}_{\text {data }}^{N_{\text {jets }}}$ of $\mathrm{W}+$ ets candidates in pretag data is subtracted the contributions from all sources other than direct $\mathrm{W}+$ ets production including non-W, electroweak diboson and top-quark.

$$
\mathrm{N}_{W+\text { jets }}^{N_{\text {jets }}} / \mathrm{N}_{\text {data }}^{N_{\text {jets }}}\left(1-\mathrm{F}_{\text {non-W }}^{N_{\text {jets }}}\right)-\mathrm{N}_{\text {diboson }}^{N_{\text {jets }}}-\mathrm{N}_{\text {top }}^{N_{\text {jets }}}
$$


To extract heavy flavor lets from $\mathrm{W}+$ ets events, the ratio between $\mathrm{W}+\mathrm{HF}$ lets and $\mathrm{W}+$ ets is obtained from Monte Carlo samples and data for the normalization.

$\mathrm{W}$ +heavy flavor production( $\mathrm{W} \mathbf{b} \overline{\mathbf{b}} \mathrm{W} \mathbf{c}, \mathrm{W}, \mathrm{c}, \mathrm{W}+\mathrm{LF}$ (light flavor)) is simulated by using Alpgen generator and PYTHIA showering. Specific heavy flavor processes are combined with light flavor production to obtain the Alpgen multi-|et samples, with the same procedure used to combine the Monte Carlo W+let samples when extracting the heavy flavor fraction [11]. The W+heavy flavor(HF) contribution to the tagged lepton+lets sample is estimated by calculating the fraction of $\mathrm{HF}$ in $\mathrm{W}+$ ets events.

$$
\mathrm{N}_{W+H F}^{\text {tag }} / \mathrm{N}_{W+\text { jets,data }}^{\text {pretag }} \times \mathrm{F}_{H F} \times \epsilon_{\text {tag }}^{W+H F} \times \mathrm{KF}
$$

where

$$
\mathrm{F}_{H F} / \frac{\mathrm{N}_{b, M C}^{W+\text { jets }}}{\mathrm{N}_{\text {jets, } M C}^{W+j e t s}}
$$

$\mathrm{F}_{H F}$ is the heavy flavor fraction of the $\mathrm{W}+\mid$ ets sample, $\epsilon_{\text {tag }}^{W+H F}$ is the tagging efficiency in the $\mathrm{W}+$ HF MC sample, $\mathrm{N}_{W+\text { jets,data }}^{\text {pretag }}$ is the number of pretag $\mathrm{W}+$ ets events in data, and $\operatorname{KF}\left(\mathrm{F}_{j, \text { data }}^{\text {b, events }} / \mathrm{F}_{j, M C}^{\text {b,events }}\right)$ is the calibration factor(K-factor) of the heavy flavor fraction. For W bb and W c c , the fitted K-factor is measured by $1.4=0.4[37]$.
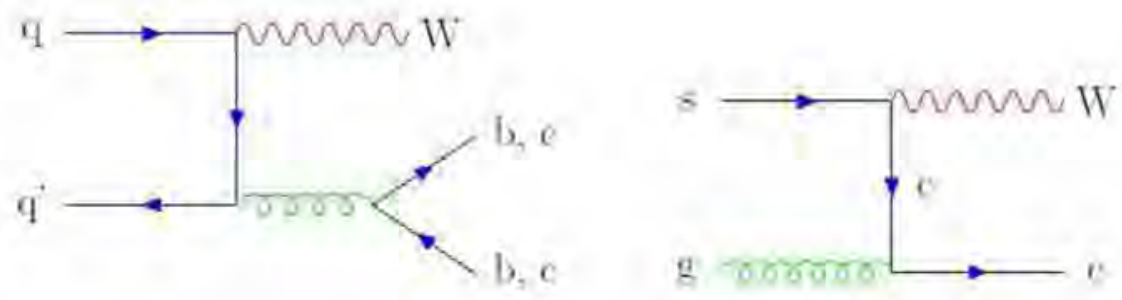

Figure 3.3. W+heavy flavor production . Wb $\mathbf{b} / \mathrm{Wc}, \mathrm{c}, \mathrm{Wc}$

\subsubsection{Mistags}

The SECVTX b-tagger is used to identify heavy-flavor quarks(mostly long-lifetime b quark) [42]. B-hadron travels a macroscopic distance before decaying, so SECVTX reconstruct a secondary vertex of a let that is well-displaced from 
primary interaction point. But there are fake tracks satisfy the secondary vertex requirements; those come from limited detector resolution, long-lived light particle decays $\left(\mathrm{K}_{s}\right.$ and $\left.\Lambda\right)$, interactions in the beampipe/detector material. The resolution effects are expected to be symmetric in the signed 2D displacement $\mathrm{L}_{x y}$ of the vector separating the secondary and primary vertices. The mistag matrix is derived from the tagging rate with negative $\mathrm{L}_{x y}$ as positive $\mathrm{L}_{x y}$ tags. Therefore, the negative $\mathrm{L}_{x y}$ rate in generic ets is an approximation of the $+\mathrm{L}_{x y}$ of light quark |ets(i.e. mistag rate). To obtain the mistag contribution of a sample of $\mathrm{W}+\mathrm{N}_{\text {jets }}$ events, the mistag probability is computed for each let in the event which is then summed over all events in the sample.

\subsubsection{Dibosons}

The diboson production WW and WZ contribute to the backgrounds according to their final states are a lepton, a neutrino and heavy-flavor lets. In the case of $Z Z$, one of $Z$ decays two leptons, so this can be a background if one lepton is lost.(Figure 3.4) The theoretical NLO cross section is predicted at ${ }^{\mathrm{p}} \overline{\mathrm{s}} / 1.96 \mathrm{TeV}$ to expect the number of diboson events in the data.
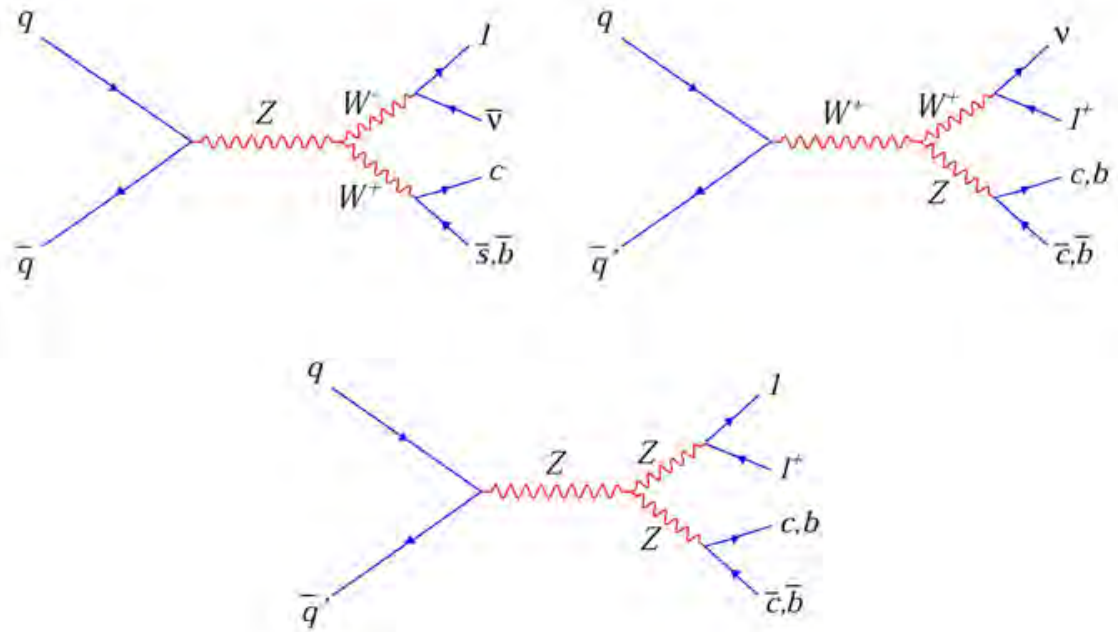

Figure 3.4. Diboson Production: WW, WZ, ZZ 


\subsubsection{Non-W}

The non-W events(QCD backgound) are modeled from the let electron and antielecton.

The let electron is a let that fakes an electron, the QCD event coming from let electron can pass the selection cut. The let is required to have $\mathrm{E}_{T}>20 \mathrm{GeV}$, $0.05<\mathrm{E}_{\text {had }} / \mathrm{E}_{e m}<0.02$, and at least four $/$ ets have to be contained in the event. The let electrons are treated as electrons and the charge of the let electron is assigned randomly. The antielectron model is considered to find electron candidates nearly passing the electron kinematic cuts. The pretag sample is used for this QCD background, "taggable" |et is defined as "tagged" |et.

\subsection{Simulation of Single-top Production includ- ing NLO effect}

In this chapter, we describe the study of using MC@NLO to generate single-topquark samples. We present a comparison between the MC@NLO samples and the official MadEvent samples currently used by the single-top group. In general we find good agreement between the two samples, but there are small number of differences.

The next-to-reading oder(NLO) QCD corrections enlarge the leading-order cross section by about $30 \%$ at Tevatron. For the analysis of single-top production, NLO results are important to have a reliable estimate of the number of events expected. The MC@NLO allows to match cross section computed at NLO in QCD with an event generator. In the case of standard $\mathrm{MC}$, a hard kinematic configuration is generated on an event-by-event basis, and it is subsequently showered and hadronized. In the case of MC@NLO, all of the hard kinematic configuration (events) are generated in advance, and stored in a file and then each event is showered and hadronized by HERWIG.

We generated event files using MC@NLO 3.3 package. We tell HERWIG that the events should be read from the event file using Les Houches interface by specifying a negative value of the process code IPROC. For the single-top production, IPROC is $-2000-\mathrm{IC}\left(\mathrm{H}_{1} \mathrm{H}_{2} \rightarrow \mathbf{t} / \overline{\mathbf{t}}+\mathrm{X}\right),-2001-\mathrm{IC}\left(\mathrm{H}_{1} \mathrm{H}_{2} \rightarrow \overline{\mathbf{t}}+\mathrm{X}\right)$, or $-2004-$ $\mathrm{IC}\left(\mathrm{H}_{1} \mathrm{H}_{2} \rightarrow \mathbf{t}+\mathrm{X}\right)$ by setting $\mathrm{IC} / 10$ and $\mathrm{IC} / 20$ for $\mathbf{s}$ - and $\mathbf{t}$-channel production. $\mathrm{H}_{1,2}$ represents hadrons, $\mathrm{p}$ or $\overline{\mathrm{p}}$.

We can set ILICODE/ 1,2,3 for the lepton identification of W-decay. Spin correlation for the decay products are therefore taken into account. 
HEPG - post-parton-showering level

We compared the MadEvent samples and MC@NLO samples at the hepg level. MadEvent single-top samples are LO s-channel, matched(LO+NLO) t-channel, and LO t-channel (for illustration purposes). Therefore, we compare the following pairs of samples. (MadEvent LO s-channel, MC@NLO s-channel), (MadEvent LO t-channel, MC@NLO t-chamnel), and (MadEvent matched t-channel, MC@NLO t-channel).

Table 3.1 shows the means of the $\mathrm{P}_{T}$ and $|\mathrm{Pz}|$ distributions for the MadEvent and MC@NLO samples. One can see that the agreement is good, with the mean values of MC@NLO distributions being consistently slightly lower than those from the MadEvent samples.

\begin{tabular}{|c|c|c|c|c|c|}
\hline \multirow{2}{*}{ Particle } & \multirow{2}{*}{ Sample } & \multicolumn{2}{|c|}{$\mathrm{P}_{T}$} & \multicolumn{2}{|c|}{$\left|\mathrm{P}_{Z}\right|$} \\
\hline & & MadEvent & MC@NLO & MadEvent & MC@NLO \\
\hline \multirow{3}{*}{ lepton } & s-chan & 40.1 & 39.24 & 25.53 & 24.98 \\
\hline & LO t-chan & 37.44 & \multirow{2}{*}{37.18} & 23.87 & \multirow{2}{*}{23,65} \\
\hline & Matched t-chan & 37.85 & & 24.09 & \\
\hline \multirow{3}{*}{ neutrino } & s-chan & 53.31 & 52 & 60.19 & 59.52 \\
\hline & LO t-chan & 50.68 & \multirow{2}{*}{49.47} & 51.16 & \multirow{2}{*}{50.05} \\
\hline & Matched t-chan & 50.49 & & 50.79 & \\
\hline \multirow{3}{*}{$b$ from $t$} & s-chan & 64.15 & 62.34 & 72.33 & 70.88 \\
\hline & LO t-chan & 60.36 & \multirow{2}{*}{59.2} & 60.6 & \multirow{2}{*}{59,14} \\
\hline & Matched t-chan & 60.39 & & 60.53 & \\
\hline \multirow{3}{*}{ top } & s-chan & 68.74 & 62.34 & 139.8 & 137.5 \\
\hline & LO t-chan & 48.29 & \multirow{2}{*}{46.24} & 119.5 & \multirow{2}{*}{115.9} \\
\hline & Matched t-chan & 49.28 & & 118,4 & \\
\hline
\end{tabular}

Table 3.1. The means of the $\mathrm{P}_{T}$ and $\mathrm{P}_{Z}$ distributions for the MadEvent and the MC@NLO samples at generator level

The compared plots of MadEvent and MC@NLO are shown Appendix B.

\section{Reconstruction level}

We compared the MadEvent single-top samples and MC@NLO single-top samples at detector-level (ie post CDF simulation, reconstruction, and trigger simulation steps) stage. We compared the distributions of $\eta, \mathrm{E}, \mathrm{Pt}$ and $|\mathrm{Pz}|$ of the top, lepton, neutrino, $\mathbf{b}$-quark from top, b-quark not from top and the light-quark, as well as top mass and Q $\times \eta$ in Appendix B. To identify the b-from-top etc we used standard HEPG matching. The neutrino $\mathrm{Pz}$ is found by imposing the $\mathrm{W}$ mass 
constraint for the lepton-neutrino invariant mass, and then taking the lower $\left|\mathrm{P}_{2}\right|$ solution of the two (or the real part if complex solutions).

Table 3.2 shows the means of the $\mathrm{P}_{T}$ and $|\mathrm{Pz}|$ distributions for the MadEvent and the MC@NLO samples.

\begin{tabular}{|c|c|c|c|c|c|}
\hline \multirow{2}{*}{ Particle } & \multirow{2}{*}{ Sample } & \multicolumn{2}{|c|}{$\mathrm{P}_{T}$} & \multicolumn{2}{|c|}{$\left|\mathrm{P}_{Z}\right|$} \\
\hline & & MadEvent & MC@NLO & MadEvent & MC@NLO \\
\hline \multirow{3}{*}{ lepton } & s-chan & 44.55 & 45.02 & 31.07 & 31.42 \\
\hline & LO t-chan & 42.24 & \multirow{2}{*}{41.9} & 30.93 & \multirow{2}{*}{29.79} \\
\hline & Matched t-chan & 42.74 & & 31.22 & \\
\hline \multirow{3}{*}{ neutrino } & s-chan & 53.77 & 53.32 & 33.23 & 32.4 \\
\hline & LO t-chan & 52.82 & \multirow{2}{*}{52.62} & 31.83 & \multirow{2}{*}{30.98} \\
\hline & Matched t-chan & 52.64 & & 31,29 & \\
\hline \multirow{3}{*}{$b$ from $t$} & s-chan & 63.04 & 62,17 & 41.49 & 40.48 \\
\hline & LO t-chan & 57.57 & \multirow{2}{*}{56.49} & 37.83 & \multirow{2}{*}{36.75} \\
\hline & Matched $t$-chan & 57.45 & & 37.41 & \\
\hline other b & s-chan & 92.73 & 89.47 & 60.98 & 59.26 \\
\hline \multirow{2}{*}{ light q } & LO t-chan & 95.41 & \multirow{2}{*}{93.42} & 123.5 & \multirow{2}{*}{125.1} \\
\hline & Matched t-chan & 93.38 & & 118.4 & \\
\hline \multirow{3}{*}{ top } & s-chan & 61.35 & 59.97 & 73.83 & 72.67 \\
\hline & LO t-chan & 54.45 & \multirow{2}{*}{53.01} & 71.32 & \multirow{2}{*}{65.66} \\
\hline & Matched t-chan & 54.03 & & 69.56 & \\
\hline
\end{tabular}

Table 3.2. The means of the $\mathrm{P}_{T}$ and $\mathrm{P}_{Z}$ distribution for MadEvent and MC@NLO samples at reconstruction level

The acceptance tables and compared plots of MC@NLO and Madevent are shown in Appendix B. 


\section{Chapter 4}

\section{Data and Event Selection}

\subsection{Data Set}

In this analysis, we used Run II CDF data of $4.8 \mathrm{fb}^{-1}$ at ${ }^{\mathrm{p}} \overline{\mathrm{s}} / 1.98 \mathrm{TeV}$ in Tevatron. Figure 4.1 shows the integrated luminosity plot during Run II (2001 2009) associated $4.8 \mathrm{fb}^{-1}$ data. The data set with each period(run range) is shown in Table 4.1.

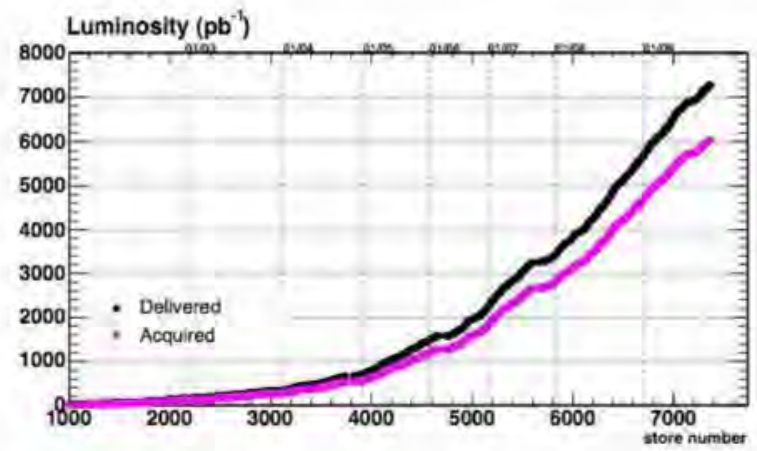

Figure 4.1. Run II(2001 2009) Integrated Luminosity; Delivered is $7.28 \mathrm{fb}^{-1}$, Acquired is $6.04 \mathrm{fb}^{-1}$, physics data is $4.8 \mathrm{fb}^{-1}$ used for this analysis

For the single top production events, we select one lepton, missing $\mathrm{E}_{T}$, two or three /ets for final states. The leptonic W decays(electron or muon and its neutrino) is only considered to reduce the backgrouds of multi-|et production (it is difficult to distinguish signal from backgrounds due to QCD multi-|et events if $\mathrm{W}$ decays into quarks).

We require the lepton $\mathrm{P}_{T}>20 \mathrm{GeV},|\eta|<1.6$, $\mid$ et $\mathrm{E}_{T}>20 \mathrm{GeV},|\eta|<2.8$, 
missing $\mathrm{E}_{T}>25 \mathrm{GeV}$ and then also remóve Dilepton, Cosmics, Conversinos, QCD events.

At least one of lets must be tagged as b-let reconstructed as a displaced secondary vertex at silicon vertex detector. The long lived $\mathbf{b}$-quark is distinguished from the light quark using b-tagging algorithm.

\begin{tabular}{|c|c|c|c|}
\hline Period & Run Range & Sample & Dataset \\
\hline p18 - p25 & $261119-277511$ & $\begin{array}{c}\text { High Pt Central Electrons } \\
\text { High Pt Plug Electrons } \\
\text { High Pt Central Muons }\end{array}$ & $\begin{array}{c}\text { bhelmm } \\
\text { bpelmm } \\
\text { bhmumm }\end{array}$ \\
\hline p14 - p17 & $252836-261005$ & $\begin{array}{c}\text { High Pt Central Electrons } \\
\text { High Pt Plug Electrons } \\
\text { High Pt Central Muons }\end{array}$ & $\begin{array}{c}\text { bhelmk } \\
\text { bpelmk } \\
\text { bhmumk }\end{array}$ \\
\hline p11 - p13 $233133-241665$ & $\begin{array}{c}\text { High Pt Central Electrons } \\
\text { High Pt Plug Electrons } \\
\text { High Pt Central Muons }\end{array}$ & $\begin{array}{c}\text { bhelm| } \\
\text { bpelm| } \\
\text { bhmum| }\end{array}$ \\
\hline p08 - p10 & $217990-233111$ & $\begin{array}{c}\text { High Pt Central Electrons } \\
\text { High Pt Plug Electrons } \\
\text { High Pt Central Muons }\end{array}$ & $\begin{array}{c}\text { bhelmi } \\
\text { bpelmi } \\
\text { bhmumi }\end{array}$ \\
\hline 0i & $203819-212133$ & $\begin{array}{c}\text { High Pt Central Electrons } \\
\text { High Pt Plug Electrons } \\
\text { High Pt Central Muons }\end{array}$ & $\begin{array}{c}\text { bhel0i } \\
\text { bpel0i } \\
\text { bhmu0i }\end{array}$ \\
\hline 0h & $190697-203799$ & $\begin{array}{c}\text { High Pt Central Electrons } \\
\text { High Pt Plug Electrons } \\
\text { High Pt Central Muons }\end{array}$ & $\begin{array}{c}\text { bhel0h } \\
\text { bpel0h } \\
\text { bhmu0h }\end{array}$ \\
\hline Od & $138425-186598$ & $\begin{array}{c}\text { High Pt Central Electrons } \\
\text { High Pt Plug Electrons } \\
\text { High Pt Central Muons }\end{array}$ & $\begin{array}{c}\text { bhel0d } \\
\text { bpel0d } \\
\text { bhmu0d }\end{array}$ \\
\hline
\end{tabular}

Table 4.1. CDF Dataset Summary , data $\sim$ p25 (luminosity $4.8 \mathrm{fb}^{-1}$ with silicon)

\subsection{Trigger}

A high $\mathrm{p}_{T}$ central lepton trigger used for the data sample. Used data sets are listed in Table 4.1.

The cetral electrons are required to pass ELECTRON_CENTRAL_18 trigger. At least one calorimeter tower with $\mathrm{E}_{T}>8 \mathrm{GeV}$ and $\mathrm{E}_{\text {had }} / \mathrm{E}_{e m}<0.125$ are requiried, and also at least one COT track with a XFT track with $\mathrm{p}_{T}>8 \mathrm{GeV}$ with hits in at least four XFT layers is demanded at level 1. At level 2, the 
CEM energy cluster have to be $\mathrm{E}_{T}>18 \mathrm{GeV}, \mathrm{E}_{\text {had }} / \mathrm{E}_{\text {em }}<0.125, \eta<1.317$ and XFT track with $\mathrm{p}_{T}>8 \mathrm{GeV}$ with hits in at least four XFT layers and stereo confirmation. At level 3 , it requires a COT track with $\mathrm{p}_{T}>8 \mathrm{GeV} / \mathrm{c}$ matched to an energy cluster in the CEM with $\mathrm{E}_{T}>18 \mathrm{GeV}$ with $\mathrm{E}_{\text {had }} / \mathrm{E}_{e m}<0.125$. The forward electrons have to pass the MET PEM trigger which is not only PEM because of higher background energy deposition from elastic collision and beam remnants in the forward region. At least one forward calorimeter tower with $\mathrm{E}_{T}>8 \mathrm{GeV}$ and $\mathrm{E}_{\text {had }} / \mathrm{E}_{e m}<0.125$ are requried and in the forward region, $\mathrm{E}_{\text {had }} / \mathrm{E}_{e m}<0.0625$ and $\mathrm{E}_{T}>15 \mathrm{GeV}$ at level 1. At level 2, an electromagnetic ob ect with $1.1<|\pi|<3.6$ and $\mathrm{E}_{T}>20 \mathrm{GeV}$ is required.

The central muon trigger MUON_CMUP18 demands a track $\mathrm{p}_{T}>18 \mathrm{GeV} / \mathrm{c}$ in COT matched to track segments of both CMU and CMP. The forward muon trigger MUON_CMX18 requires a COT track with $\mathrm{P}_{T}>18 \mathrm{GeV} / \mathrm{c}$ has to be matched with reconstructed stubs in CMX.

\subsection{Selection for W Candidates}

\subsubsection{Lepton}

\section{Electron Candidate Selection}

The selection cuts on electron candidates are required to increase the sample purity. The energy deposited in the electromagnetic calorimeter have to match a track from the tracking system and also electron has to be distiguished from other particles(charged hadrons or photons). For the central electron, $\mathrm{E}_{T}>20$ $\mathrm{GeV}$ in CEM has to match with a reconstructed track $\mathrm{P}_{T} \geq \mathrm{GeV}$ and Isolation has to be less than 0.1. Table 4.2 lists central electron candidate requirements. For the forward electron, $\mathrm{E}_{T}>20 \mathrm{GeV}$ in PEM is required. Table 4.3 shows plug electron candidate requirements.

- $\mathrm{E}_{T}$. Total transverse energy of towers in the cluster.

- $\mathrm{P}_{T}$. Transverse momentum of the COT track pointing to the cluster.

- E/p . Ratio of the energy in the EM cluster to the associated track's transverse momentum in COT. This is useful to re/ect the accidental overlap of charged hadrons and photons.

- $\mathrm{E}_{\text {had }} / \mathrm{E}_{e m}$. Ratio of energies measured in the hadronic calorimeter of the CHA and WHA to that from the EM calorimeter(CEM). An real electron has a smaller value of $\mathrm{E}_{h a d} / \mathrm{E}_{e m}$. 


\begin{tabular}{|c|c|}
\hline \multicolumn{2}{|c|}{ Central Tight Electrons } \\
\hline Geometric & Fiducial in CEM \\
$\mathrm{E}_{T}$ & $\geq 20 \mathrm{GeV}$ \\
Track $\left|4 \mathrm{z}_{0}\right|$ & $\leq 60 \mathrm{~cm}$ \\
Track $\mathrm{P}_{T}$ & $\geq 10 \mathrm{GeV} / \mathrm{c}$ \\
$\mathrm{E}_{\text {had }} / \mathrm{E}_{\text {em }}$ & $\leq 0.055+0.00045^{*} \mathrm{E}$ \\
$\mathrm{L}_{\text {shr }}$ & $\leq 0.2$ \\
$\mathrm{E} / \mathrm{P}$ if $\mathrm{P}_{T} \leq 50 \mathrm{GeV} / \mathrm{c}$ & $\leq 2$ \\
$|4 \mathrm{z}|$ & $\leq 3 \mathrm{~cm}$ \\
$\mathrm{Q} \times 4 \mathrm{x}$ & $\geq-3.0$ and $\leq 1.5 \mathrm{~cm}$ \\
$\chi_{\text {strip }}^{2}$ & $\leq 10$ \\
$\#$ axial SL & $\geq 3$ \\
\# stereo SL & $\geq 2$ \\
Isolation & $\leq 0.1$ \\
\hline
\end{tabular}

Table 4.2. Cental Electron Identification Requirements

\begin{tabular}{|c|c|}
\hline \multicolumn{2}{|c|}{ Plug Tight Phoenix Electrons } \\
\hline $\mathrm{E}_{T}$ & $\geq 20 \mathrm{GeV}$ \\
Track $\left|4 \mathrm{z}_{0}\right|$ & $\leq 60 \mathrm{~cm}$ \\
\# Silicon Hits & $\geq 3$ \\
PES $2 \mathrm{D} \mathrm{Eta}$ & $1.2 \leq|\eta| \leq 2.8$ \\
$\mathrm{E}_{\text {had }} / \mathrm{E}_{\text {em }}$ & $\leq 0.05$ \\
PEM $3 \times 3 \chi^{2}$ & $\leq 10$ \\
PES $5 \times 9 \mathrm{U}$ & $\geq 0.65$ \\
PES $5 \times 9 \mathrm{~V}$ & $\geq 0.65$ \\
Isolation & $\leq 0.1$ \\
$|4 \mathrm{R}|$ & $\leq 3.0 \mathrm{~cm}$ \\
\hline
\end{tabular}

Table 4.3. Plug Electron Identification Requirements 
- $\left|4 \mathrm{z}_{0}\right| .4 \mathrm{z}_{0}$ of the eletron track is required to be within $60 \mathrm{~cm}$ of the nominal interaction point.

- $\mathrm{L}_{\text {shr }}$. A measure of the difference between lateral development of an electron candidate's shower profile and the expected profile as measured in test beam data. Most electrons deposit the energy on a single CEM tower because the lateral size of electromagnetic shower is smaller than the tower size. However, an overlap event of charged hadron and multiple photons can leave extra energies on towers ad|acent to a seed tower by multi-photon hit.

- ] axial/stereo SL . Number of axial/stereo super laters with hits associated to the COT track, pointing to the EM cluster.

- Isolation. The amount of energy that is deposited in a cone around a track for distinguishing between an isolated particle and a particle in a let. Isolation is defined as $\left(\mathrm{E}_{T}^{\text {cone }}-\mathrm{E}_{T}^{\text {cluster }}\right) / \mathrm{E}_{T}^{\text {cluster }}$, where $\mathrm{E}_{T}^{\text {cone }}$ is the $\mathrm{E}_{T}$ of all towers in a cone of $4 \mathrm{R} / 0.4$ centered on the electron cluster and $\mathrm{E}_{T}^{\text {cluster }}$ is the total $\mathrm{E}_{T}$ of towers included in the cluster.

\section{Muon Candidate Selection}

The muon candidates are required a COT track $\mathrm{p}_{T}>20 \mathrm{GeV} / \mathrm{c}$ that pointed to a track segment in a muon chamber. For central muon, track segments are detected in both CMU and CMP(this defines CMUP muons), or in the CMX. Table 4.4 shows the muon identification requirements.

\subsubsection{Neutrino}

The neutrino escape the detector, so the missing energy of transverse direction is defind as $\mathrm{E}_{T}$ of neutrino and $\mathrm{Pz}$ of neutrino is calculated by using $\mathrm{W}$ mass constraint. First, missing transverse energy is summed over all calorimeter towers.

$$
\mathrm{E}_{T} /-\sum_{i} \mathrm{E}_{T}^{i} \mathrm{n}_{i}
$$

where $\mathrm{E}_{T}^{i}$ is the transverse energy deposited in the ith calorimeter tower, $\hat{\mathbf{n}}_{i}$ is a unit vector perpendicular to the beam axis pointing to the ith calorimeter tower. The muons leave a minimum amount of energy in the calorimeter, so muon $\mathrm{E}_{T}$ can be measured from muon $\mathrm{P}_{T}$ (mass is negligible compared to the kinematic energy). In this case, $\mathbb{F}_{T}$ is calculated as $\left(\mathbb{F}_{T}^{c o r r}\right)_{x y} /\left(\mathbb{F}_{T}^{r a w}\right)_{x y}-\left(\mathrm{p}_{\mu t}\right)_{x y}$ $+\left(E_{T}^{\text {depos }}\right)_{x y}$. The small amount energy deposited in the calorimeter by the 


\begin{tabular}{|c|c|}
\hline \multicolumn{2}{|c|}{ Central Muon Candidate } \\
\hline Geometric & Fiducial in CMUP or CMX \\
$\mathrm{P}_{T}$ & $>20 \mathrm{GeV} / \mathbf{c}$ \\
$\mathrm{E}_{e m}$ & $<2 \mathrm{GeV}+\max \left(0,0.0115^{*}(\mathrm{p}-100)\right)$ \\
$\mathrm{E}_{\text {had }}$ & $<6 \mathrm{GeV}+\max \left(0,0.0280^{*}(\mathrm{p}-100)\right)$ \\
Tracks with no silicon hits & $|\mathrm{d} 0|<0.2 \mathrm{~cm}$ \\
Tracks with silicon hits & $|\mathrm{d} 0|<0.02 \mathrm{~cm}$ \\
(if CMUP) $|4 \mathrm{x}|_{\text {CMU }}$ & $<7.0 \mathrm{~cm}$ \\
(if CMUP) $|4 \mathrm{x}|_{\text {CMP }}$ & $<5.0 \mathrm{~cm}$ \\
(if CMX) $|4 \mathrm{x}|_{\text {CMX }}$ & $<6.0 \mathrm{~cm}$ \\
4 zo & $<60 \mathrm{~cm}$ \\
\# axial SL & $\geq 3$ \\
\# stereo SL & $\geq 2$ \\
Isolation & $<0.1$ \\
\hline
\end{tabular}

Table 4.4. Muon Identification Requirements

muons is subtrated. For $\mathrm{Pz}$ of neutrino, we set $\mathrm{W}$ mass as $80.4 \mathrm{GeV}$ and use $\mathrm{m}_{W} / \sqrt{\left(\mathrm{p}_{l}+\mathrm{p}_{\nu}\right)^{2}}$.

\subsection{Jet Reconstruction}

The lets are identified using a cone-clustering algorithm with cone size $4 \mathrm{R}$ / $\sqrt{4 \eta^{2}+4 \phi^{2}}$. The sum of energy deposited in a cone with radius 0.4 which is centered at seed cluster is refered to as 'raw' energy of let. This measured 'raw' energy is underestimated compared to the original parton |et. Therefore, the measured let energy have to be corrected to the parent parton energy as the following let correction procedures.

\section{Jet Corrections}

The let corrections from several different sources are performed with different 'levels'. The sources are the respose of the calorimeter to different particles, non-linearity response of the calorimeter to the particle energies, spectator interactions, energy radiated outside the let clustering algorithm and so on. The let energy correction has the following eight levels.

- Level 0. Online and Offline calibrations

This correction sets the calorimeter energy scale, CEM scale set to $Z \rightarrow$ ee 
peak(91.2GéV). The time dependence and PMT gains are tracked via the inclusive $8 \mathrm{GeV}$ electron data. CHA scale is set to Run 1 MIP(Minimum Ionizing Particles) peak and tower gain varitions are tracked via J/Psi muons and min-bias rates. WHA scale is tracked with source calibrations. PEM/PHA PMT gains drop with integrated charge, and are tracked with offline LERs from frequent laser calibrations. Scale shifts are tracked via a combination of source calibraions and $\mathrm{CP} Z \rightarrow$ ee data.

\section{- Level 1. Eta Dependence}

This correction is the "eta-dependent of relative correction" to correct measured let energy uniformly along eta. The $\eta$-dependent corrections are performed using the "dilet balancing method". The transverse energy of the two lets in di|et events should be balanced. This property is used to scale lets outside the $0.2<|\eta|<0.6$ region to $\mid$ ets inside the region because CEM and CHA are well understood calorimeters.

\section{- Level 2}

It was for removing time dependence of the calorimeter PMTs. This is now done at the tower level in level0. Currently, this correction is not being applied.

\section{- Level 3}

This correction took care of the differences in scale between Run I vs Run II. In Gen4 it also covered some difference between MC and data observed in photon + ets events. Now this difference is covered by other uncertainties, so it is not being applied.

\section{- Level 4. Multiple Interactions}

At high instantaneous luminosities more than one pp interaction occurs in the same bunch crossing. The energy from different $\mathrm{p} \overline{\mathrm{p}}$ interaction inside the let cluster increases measured let energy, so this extra energy should be subtracted. The average transverse energy in a cone is measured using minimum bias data sample and this data are parameterized as a function of the number of vertices in the event. The transverse energy in a cone, which is defined using a seed tower randomly selected in the central calorimeter region $0.2<|\eta|<0.6$, is measured as a function of the number of vertices for three cone size. 
The absolute correction carry out the transformation of the meausured |et energy into the energy correponding to the underlying particle let. Since the calorimeter simulation is optimized to reproduce the measured single particle response, we rely on the simulation for correction over a wide range of let transverse momentum.

\section{- Level 6. Underlying Events}

The particle let not related to the actual mother partons of the hard interaction of the let is called "Underlying Event"; such as the initial state radiation(ISR) or spectator partons with color connection to the other partons of the proton. Therefore, the UE energy needs to be subtracted from the particle-level |et energy.

\section{- Level 7. Out of Cone Corrections}

A fraction of the parton energy can be lost from the let cone due to final state gluon radiation(FSR) and this energy is called "Out-of-Cone" energy. Final State Radiation and hadronization are correlated with the primary let direction and the energy. The corrections are determined from MC simulation at particle generator level. The systematic uncertainty of UE and OOC correction is derived from comparisions of the energy measured in calorimeter towers in a let cone with the simulation of Phthia and Herwig.

\subsection{B Jet Idendification}

The idenfication of (ets from b quark (or b-tagging) is important to reconstruct the top quark.

The $b$-lets have the different features from light flavor and charm |ets.the long lifetime of the $\mathbf{b}$ quark, large mass of $\mathrm{B}$ hadrons and energetic semileptonic decay of $\mathrm{B}$ hadrons.

To idenfify $\mathbf{b}$ let, we use CDF's secondary vertex btagger(b-tagging tool) using these distinguishing features.

The b-quark hadronizes almost immediately to form a let of particles; a B meson $\left(\mathrm{B}^{0}, \mathrm{~B}^{ \pm}, \mathrm{B}_{s}^{0}\right)$ or a $\mathrm{B}$ baryon $\left(\Lambda_{B}\right)$ is included in a et. The $\mathrm{B}$ hadron usually has most of the original b-quark momentum and has a long lifetime. Before decaying particles, the $\mathrm{B}$ hadrons moved a macroscopic distance away from the primary intercation point due to this long lifetime and large boost. Each track's impact parameter $\left(\mathrm{d}_{0}\right)$ is measured with respect to a primary pp interaction posi- 
tion(or primary vertex). The silicon tracks within let are required to have $\mathrm{p}_{T}>$ $0.5 \mathrm{GeV}, \mathrm{d}_{0}$ significance $\mathrm{S}_{d_{0}} /\left|\frac{d_{0}}{\delta_{d_{0}}}\right|>2.0$, minimum number of hits in the silicon tracking detector and finally the tracks must not exceed a maximum $d_{0}$ requirement. The selected tracks are ordered in $\mathrm{p}_{T}$ and then a 2-track "seed" vertex is searched among those tracks. If a seed vertex is found, the remaining tracks are considered for vertexing with the seed tracks. The vertex $\chi^{2}$ is calculated after attaching all qualifying tracks to the vertex, and tracks are iteratively pruned from the vertex if they contribute too much to the overall $\chi^{2}$. Having three or more tracks is final cuts, including removal of vertices from material, nuclear interactions, long-lived light flavor hadrons and so on. Finally the vertex is required to have $\mathrm{S}_{L_{x y}}>7.5$, where $\mathrm{S}_{L_{i x y}} /\left|\mathrm{L}_{x t} / \delta_{L_{x y}}\right| . \mathrm{L}_{x y}$ is the $2 \mathrm{D}$ decay length of the fitted vertex with respect to the primary. The let is defined as "tagged" if a secondary vertex is found as either followng criterion. The tag is called "positive" if the product of the 2D displacement vector from primary vertex to the secondary vertex and the let's momentum is positive, and the tag is called "negative" if that product is negative. Most b-tags are reconstructed in the "positive" tag, and "negative" tagged lets are considered as mistags.

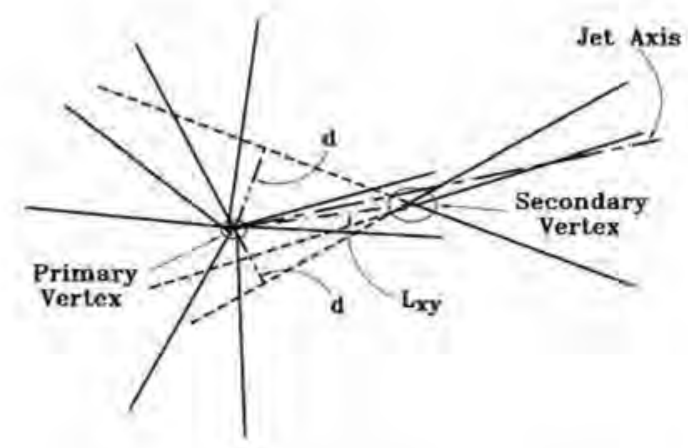

Figure 4.2. Primary and Secondary vertices of tracks of |et

\subsection{Data Reduction and Final Candidate Sample}

For single-top analysis, the proper modeling of the signal and background processes is important, as well as precise estimate of the signal and background components of the data. We can select more pure signal events reducing backgrounds by applying the $\mathrm{W}$ selection and |et requirement explained previous sections. For 
further event selection requirement, the event vetoes also contribute to reduce the background events.

\section{Dilepton Veto}

We require one lepton per event for single-top analysis, so the events having additional lepton are removed. Also the events coming from $t \bar{t}$ or diboson background can be reduced from this selection cut.

z Vertex Cut

The recontructed primary $\mathrm{z}$ vertex of the events have to be put in a region within $\pm 60 \mathrm{~cm}$ of the center of the detector.

\section{$Z$ Boson Veto}

The events having leptons supposed to come from $Z$ boson is removed. The invariant mass of two leptons is consistent with a $Z$ boson mass as $76 \mathrm{GeV} / \mathbf{c}^{2} \leq$ $\mathrm{M}_{Z} \leq 106 \mathrm{GeV} / \mathbf{c}^{2}$.

QCD Veto

The events from QCD multilet(non-W) is also removed. Because the QCD events do not have $\mathbb{F}_{T}$, the events passing $\mathbb{F}_{T}$ cut come from lost or mismeasured lets. The assumed QCD events has following features. The measured $\mathbb{E}_{T}$ is low, $\mathrm{E}_{T}$ lines up with the direction of the leading let. Thus, the QCD cut removes the events which $\mathrm{F}_{T}<30 \mathrm{GeV}$ and the angle between the $\mathrm{F}_{T}$ and the leading let is less than 0.5 or greater than 2.5 radians. The additional cuts for each lepton category are explained in [53].

The expected number of signal and background processes are obtained from CDF's Method II. The theoretically well known process's expectation is decided using simulated events.

The event selection tables of signal events of s-channel(stop00) and one of $\mathrm{W} \mathbf{b}$ backgrounds (btop0w) for each lepton category, for example, are shown in Table $4.5 \sim 4.12$. The event efficiency of s-channel is $14 \%$ for 2 et and $7.2 \%$ for $3 \mid$ et and t-channel is $9.5 \%$ for $2 \mid$ et and $5.2 \%$ for $3 \mid$ et events. The efficiency of $\mathbf{t t}$ is $14 \%$ for 2 let and $12.8 \%$ for 3 |et, Wbb is $3 \%$ for 2 et and $4 \%$ for 3 et, W $\bar{c} \bar{c}$ is $1 \%$ for 2 et and $1 \%$ for $3 \mid$ et and $\mathrm{W}+\mathrm{LF}$ is $0.2 \%$ for $2 \mid$ et and $0.57 \%$ for $3 \mid$ et. The efficiency of Diboson is $0.56 \%$ for 2 |et and $0.19 \%$ for 3 et and $Z+$ ets is $0.1 \%$ for 2 et and $0.2 \%$ for 3 |et. As it shows, the efficiency of single-top signal processes is much higher than the efficiency of the backgrounds except $t \bar{t}$ background.

The final expectation number of signal and backgrounds and the number of observed data are shown in Table 4.13. 


\begin{tabular}{|c|c|c|c|c|c|c|}
\hline CEM & 0Jet & 1Jet & 2Jet & 3Jet & 4+Jet & Total \\
\hline Stage 0 Initial & 9353 & 130201 & 418316 & 266628 & 93675 & 918173 \\
Stage 1 Good Run & 8870 & 124129 & 397188 & 249766 & 87422 & 867375 \\
Stage 2 Trigger & 8870 & 124129 & 397188 & 249766 & 87422 & 867375 \\
Stage 3 > / TLep & 1540 & 21244 & 62256 & 25088 & 8106 & 118234 \\
Stage 4 Met & 1321 & 17786 & 52257 & 21249 & 6996 & 99609 \\
Stage 5 Iso & 1321 & 17441 & 49261 & 17040 & 4816 & 89879 \\
Stage 6 Dilep & 1311 & 17259 & 48899 & 16943 & 4787 & 89199 \\
Stage 7 Z & 1300 & 17134 & 48561 & 16808 & 4750 & 88553 \\
Stage 8 Conv & 1300 & 17067 & 47994 & 15185 & 4059 & 85605 \\
Stage 9 Diff Z & 1300 & 17067 & 47990 & 15184 & 4058 & 85599 \\
Stage 10 QCD & 1300 & 16249 & 45092 & 14154 & 4058 & 80853 \\
Stage 11 Si Good Run & 1300 & 16249 & 45092 & 14154 & 4058 & 80853 \\
Stage 12 Taggable & 0 & 12278 & 41684 & 13491 & 3913 & 71366 \\
Stage 13 > / 1 +b-tag & 0 & 5962 & 26330 & 8444 & 2460 & 43196 \\
\hline
\end{tabular}

Table 4,5. CEM Acceptance Table of s-channel Sample(stop00)

\begin{tabular}{|c|c|c|c|c|c|c|}
\hline PHX & 0Jet & 1Jet & 2.Jet & 3Jet & 4+Jet & Total \\
\hline Stage 0 Initial & 9353 & 130201 & 418316 & 266628 & 93675 & 918173 \\
Stage 1 Good Run & 8870 & 124129 & 397188 & 249766 & 87422 & 867375 \\
Stage 2 Trigger & 8870 & 124129 & 397188 & 249766 & 87422 & 867375 \\
Stage 3 > 1 TLep & 524 & 6353 & 15828 & 5028 & 1362 & 29095 \\
Stage 4 Met & 453 & 5212 & 12951 & 4186 & 1130 & 23932 \\
Stage 5 Iso & 453 & 5175 & 12615 & 3685 & 925 & 22853 \\
Stage 6 Dilep & 447 & 5123 & 12535 & 3659 & 918 & 22682 \\
Stage 7 Z & 445 & 5090 & 12441 & 3632 & 907 & 22515 \\
Stage 8 Conv & 445 & 5090 & 12441 & 3632 & 907 & 22515 \\
Stage 9 Diff Z & 445 & 5090 & 12440 & 3632 & 907 & 22514 \\
Stage 10 QCD & 445 & 4716 & 10748 & 3099 & 907 & 19915 \\
Stage 11 Si Good Run & 445 & 4716 & 10748 & 3099 & 907 & 19915 \\
Stage 12 Taggable & 0 & 3607 & 10206 & 3038 & 897 & 17748 \\
Stage 13 >/ 1 +b-tag & 0 & 1707 & 6339 & 1927 & 575 & 10548 \\
\hline
\end{tabular}

Table 4.6. PHX Acceptance Table of s-channel Sample(stop00) 


\begin{tabular}{|c|c|c|c|c|c|c|}
\hline CMUP & 0Jet & 1Jet & 2Jet & 3Jet & 4+Jet & Total \\
\hline Stage 0 Initial & 9353 & 130201 & 418316 & 266628 & 93675 & 918173 \\
Stage 1 Good Run & 8870 & 124129 & 397188 & 249766 & 87422 & 867375 \\
Stage 2 Trigger & 8870 & 124129 & 397188 & 249766 & 87422 & 867375 \\
Stage 3 > / TLep & 1005 & 13342 & 39348 & 13453 & 3804 & 70952 \\
Stage 4 Met & 804 & 10970 & 32518 & 11128 & 3172 & 58592 \\
Stage 5 Iso & 751 & 10244 & 29822 & 9623 & 2558 & 52998 \\
Stage 6 Dilep & 743 & 10173 & 29592 & 9560 & 2535 & 52603 \\
Stage 7 Z & 742 & 10129 & 29479 & 9517 & 2523 & 52390 \\
Stage 8 Conv & 742 & 10129 & 29479 & 9517 & 2523 & 52390 \\
Stage 9 Diff Z & 742 & 10128 & 29478 & 9516 & 2523 & 52387 \\
Stage 10 QCD & 742 & 9488 & 28659 & 9246 & 2523 & 50658 \\
Stage 11 Si Good Run & 742 & 9488 & 28659 & 9246 & 2523 & 50658 \\
Stage 12 Taggable & 0 & 7162 & 26519 & 8795 & 2434 & 44910 \\
Stage 13 > / 1 +b-tag & 0 & 3464 & 16722 & 5427 & 1572 & 27185 \\
\hline
\end{tabular}

Table 4.7. CMUP Acceptance Table of s-channel Sample(stop00)

\begin{tabular}{|c|c|c|c|c|c|c|}
\hline CMX & 0Jet & 1Jet & 2Jet & 3Jet & 4+Jet & Total \\
\hline Stage 0 Initial & 9353 & 130201 & 418316 & 266628 & 93675 & 918173 \\
Stage 1 Good Run & 8781 & 122713 & 392743 & 247012 & 86445 & 857694 \\
Stage 2 Trigger & 8781 & 122713 & 392743 & 247012 & 86445 & 857694 \\
Stage 3 >/ 1 TLep & 464 & 6103 & 17390 & 5814 & 1571 & 31342 \\
Stage 4 Met & 380 & 4992 & 14393 & 4837 & 1316 & 25918 \\
Stage 5 Iso & 351 & 4674 & 13183 & 4171 & 1079 & 23458 \\
Stage 6 Dilep & 345 & 4634 & 13091 & 4142 & 1071 & 23283 \\
Stage 7 Z & 343 & 4617 & 13047 & 4117 & 1065 & 23189 \\
Stage 8 Conv & 343 & 4617 & 13047 & 4117 & 1065 & 23189 \\
Stage 9 Diff Z & 343 & 4617 & 13046 & 4116 & 1065 & 23187 \\
Stage 10 QCD & 343 & 4515 & 12700 & 3999 & 1065 & 22622 \\
Stage 11 Si Good Run & 343 & 4515 & 12700 & 3999 & 1065 & 22622 \\
Stage 12 Taggable & 0 & 3374 & 11937 & 3832 & 1039 & 20182 \\
Stage 13 >/ 1 +b-tag & 0 & 1630 & 7528 & 2407 & 644 & 12209 \\
\hline
\end{tabular}

Table 4.8. CMX Acceptance Table of s-channel Sample(stop00) 


\begin{tabular}{|c|c|c|c|c|c|c|}
\hline CEM & 0Jet & 1.Jet & 2Jet & 3.Jet & 4+.Jet & Total \\
\hline Stage 0 Initial & 515254 & 708112 & 270256 & 46603 & 2314 & 1542539 \\
Stage 1 Good Run & 501996 & 662337 & 249283 & 42357 & 2104 & 1458077 \\
Stage 2 Trigger & 501996 & 662337 & 249283 & 42357 & 2104 & 1458077 \\
Stage 3 > / 1 TLep & 243906 & 156610 & 41891 & 4059 & 243 & 446709 \\
Stage 4 Met & 206455 & 124699 & 34099 & 3524 & 202 & 368979 \\
Stage 5 Iso & 206455 & 107764 & 26542 & 1929 & 108 & 342798 \\
Stage 6 Dilep & 206392 & 107589 & 26461 & 1921 & 108 & 342471 \\
Stage 7 Z & 205883 & 107122 & 26289 & 1901 & 107 & 341302 \\
Stage 8 Conv & 205883 & 100408 & 22757 & 1079 & 63 & 330190 \\
Stage 9 Diff Z & 205849 & 100395 & 22755 & 1079 & 63 & 330141 \\
Stage 10 QCD & 205849 & 97689 & 22064 & 1036 & 63 & 326701 \\
Stage 11 Si Good Rum & 205849 & 97689 & 22064 & 1036 & 63 & 326701 \\
Stage 12 Taggable & 0 & 66352 & 19086 & 955 & 59 & 86452 \\
Stage 13 > 1 +b-tag & 0 & 27900 & 10992 & 549 & 39 & 39480 \\
\hline
\end{tabular}

Table 4.9. CEM Acceptance Table of Wbb Sample(btop0w)

\begin{tabular}{|c|c|c|c|c|c|c|}
\hline PHX & 0Jet & 1Jet & 2Jet & 3Jet & 4+Jet & Total \\
\hline Stage 0 Initial & 9353 & 130201 & 418316 & 266628 & 93675 & 918173 \\
Stage 1 Good Run & 8870 & 124129 & 397188 & 249766 & 87422 & 867375 \\
Stage 2 Trigger & 8870 & 124129 & 397188 & 249766 & 87422 & 867375 \\
Stage 3 > / TLep & 524 & 6353 & 15828 & 5028 & 1362 & 29095 \\
Stage 4 Met & 453 & 5212 & 12951 & 4186 & 1130 & 23932 \\
Stage 5 Iso & 453 & 5175 & 12615 & 3685 & 925 & 22853 \\
Stage 6 Dilep & 447 & 5123 & 12535 & 3659 & 918 & 22682 \\
Stage 7 Z & 445 & 5090 & 12441 & 3632 & 907 & 22515 \\
Stage 8 Conv & 445 & 5090 & 12441 & 3632 & 907 & 22515 \\
Stage 9 Diff Z & 445 & 5090 & 12440 & 3632 & 907 & 22514 \\
Stage 10 QCD & 445 & 4716 & 10748 & 3099 & 907 & 19915 \\
Stage 11 Si Good Run & 445 & 4716 & 10748 & 3099 & 907 & 19915 \\
Stage 12 Taggable & 0 & 3607 & 10206 & 3038 & 897 & 17748 \\
Stage 13 >/ 1 +b-tag & 0 & 1707 & 6339 & 1927 & 575 & 10548 \\
\hline
\end{tabular}

Table 4.10. PHX Acceptance Table of Wbb Sample(btop0w) 


\begin{tabular}{|c|c|c|c|c|c|c|}
\hline CMUP & 0Jet & 1Jet & 2Jet & 3Jet & 4+Jet & Total \\
\hline Stage 0 Initial & 9353 & 130201 & 418316 & 266628 & 93675 & 918173 \\
Stage 1 Good Run & 8870 & 124129 & 397188 & 249766 & 87422 & 867375 \\
Stage 2 Trigger & 8870 & 124129 & 397188 & 249766 & 87422 & 867375 \\
Stage 3 > / TLep & 1005 & 13342 & 39348 & 13453 & 3804 & 70952 \\
Stage 4 Met & 804 & 10970 & 32518 & 11128 & 3172 & 58592 \\
Stage 5 Iso & 751 & 10244 & 29822 & 9623 & 2558 & 52998 \\
Stage 6 Dilep & 743 & 10173 & 29592 & 9560 & 2535 & 52603 \\
Stage 7 Z & 742 & 10129 & 29479 & 9517 & 2523 & 52390 \\
Stage 8 Conv & 742 & 10129 & 29479 & 9517 & 2523 & 52390 \\
Stage 9 Diff Z & 742 & 10128 & 29478 & 9516 & 2523 & 52387 \\
Stage 10 QCD & 742 & 9488 & 28659 & 9246 & 2523 & 50658 \\
Stage 11 Si Good Run & 742 & 9488 & 28659 & 9246 & 2523 & 50658 \\
Stage 12 Taggable & 0 & 7162 & 26519 & 8795 & 2434 & 44910 \\
Stage 13 > 1 1 +b-tag & 0 & 3464 & 16722 & 5427 & 1572 & 27185 \\
\hline
\end{tabular}

Table 4.11. CMUP Acceptance Table of Wbb Sample(btop0w)

\begin{tabular}{|c|c|c|c|c|c|c|}
\hline CMX & 0Jet & 1Jet & 2.Jet & 3Jet & 4+Jet & Total \\
\hline Stage 0 Initial & 9353 & 130201 & 418316 & 266628 & 93675 & 918173 \\
Stage 1 Good Run & 8781 & 122713 & 392743 & 247012 & 86445 & 857694 \\
Stage 2 Trigger & 8781 & 122713 & 392743 & 247012 & 86445 & 857694 \\
Stage 3 > 1 TLep & 464 & 6103 & 17390 & 5814 & 1571 & 31342 \\
Stage 4 Met & 380 & 4992 & 14393 & 4837 & 1316 & 25918 \\
Stage 5 Iso & 351 & 4674 & 13183 & 4171 & 1079 & 23458 \\
Stage 6 Dilep & 345 & 4634 & 13091 & 4142 & 1071 & 23283 \\
Stage 7 Z & 343 & 4617 & 13047 & 4117 & 1065 & 23189 \\
Stage 8 Conv & 343 & 4617 & 13047 & 4117 & 1065 & 23189 \\
Stage 9 Diff Z & 343 & 4617 & 13046 & 4116 & 1065 & 23187 \\
Stage 10 QCD & 343 & 4515 & 12700 & 3999 & 1065 & 22622 \\
Stage 11 Si Good Run & 343 & 4515 & 12700 & 3999 & 1065 & 22622 \\
Stage 12 Taggable & 0 & 3374 & 11937 & 3832 & 1039 & 20182 \\
Stage 13 > / 1 +b-tag & 0 & 1630 & 7528 & 2407 & 644 & 12209 \\
\hline
\end{tabular}

Table 4.12. CMX Acceptance Table of Wbb Sample(btop0w) 


\begin{tabular}{|c|c|c|}
\hline & $2 \mid$ ets & $3 \mid$ ets \\
\hline s-channel & $72.7 \pm 10.5$ & $23.2 \pm 3.3$ \\
t-channel & $111.4 \pm 16.5$ & $32.4 \pm 4.8$ \\
Wbb & $906.9 \pm 273.4$ & $259.5 \pm 78.4$ \\
Wcc + Wc & $755.25 \pm 232.9$ & $192.1 \pm 59.2$ \\
Mistag & $681.6 \pm 95.4$ & $195.3 \pm 27.9$ \\
ttbar & $246.75=35.8$ & $559.65 \pm 81$ \\
Z+lets & $41.85 \pm 6.4$ & $16.2 \pm 2.4$ \\
Diboson & $117.45 \pm 12.15$ & $37.1 \pm 3.9$ \\
non-W & $123.3 \pm 49.35$ & $49.8 \pm 19.9$ \\
\hline Total Signal & $184.1 \pm 27.0$ & $55.6 \pm 8.1$ \\
\hline Total Prediction & $3057 \pm 519.3$ & $1365.3 \pm 162.4$ \\
\hline Observed in Data & 2920 & 1300 \\
\hline
\end{tabular}

Table 4.13. Data and Background for single-top production with $4.8 \mathrm{fb}^{-1}$

After the selection cut, the plots of $\mathrm{Pt}$ (transeverse momentum) of lepton, missing Et, Pt of tagged let(to select b-quark from top) and $\mathrm{E}$ (energy) of lepton, $\mathrm{E}$ of neurino, $\mathrm{E}$ of tagged /et are shown in Figure $4.3 \sim 4.8$.

In the case of the tagged let, the transverse momentum of single-top (s-channel, t-channel) and $t \bar{t}$ are higher than $W b \bar{b} W c(\overline{\mathbf{c}})$ and mistag. Also the peak of energy of single-top and $t \bar{t}$ are higher than other backgrounds. 

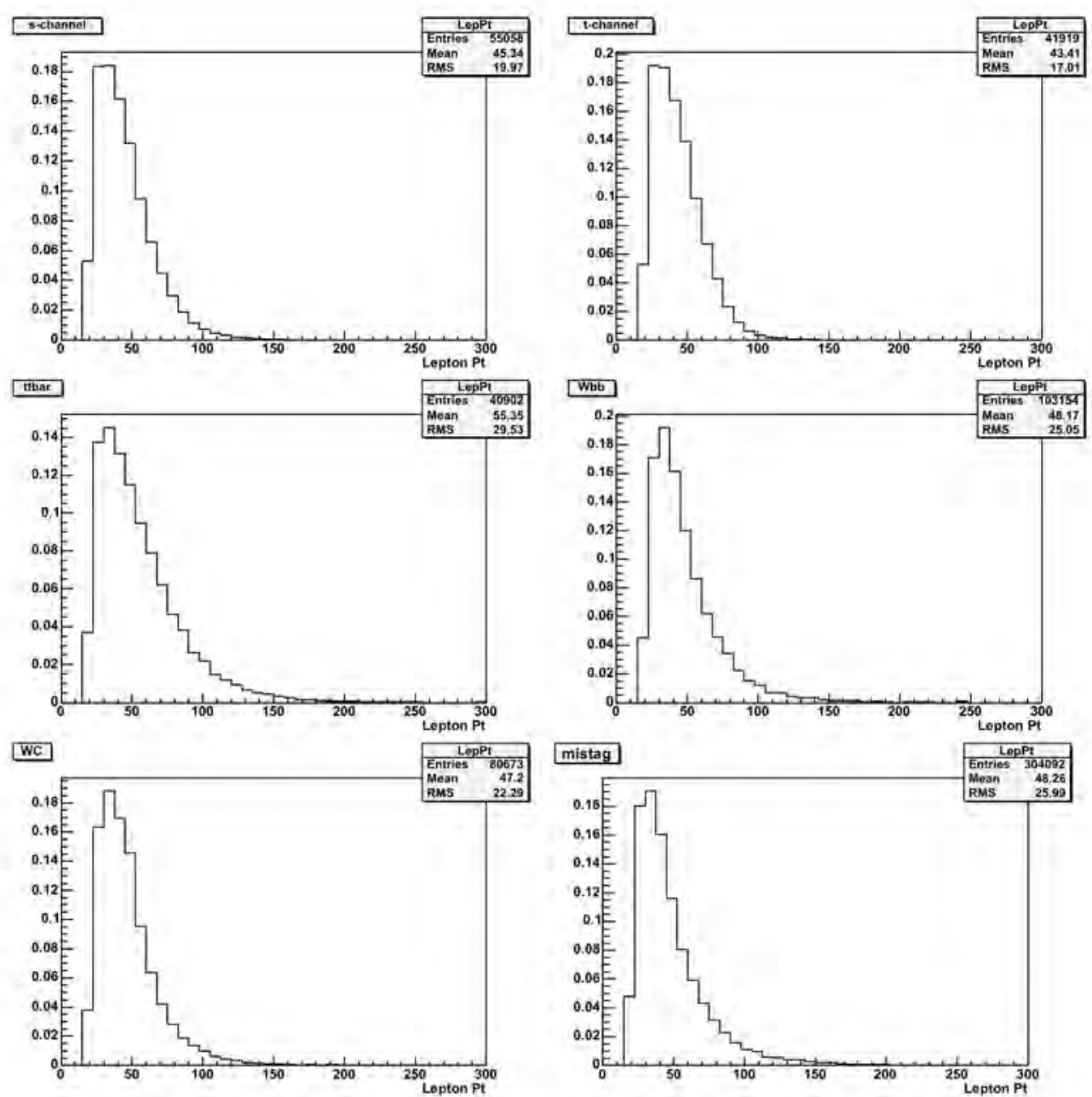

Figure 4.3. Lepton $\mathrm{Pt}$ of $\mathbf{s}$-channel, t-channel, $\mathbf{t t}, \mathrm{Wba} \mathbf{b} \mathbf{~} \mathbf{c}(\overline{\mathbf{c}})$ and mistag after selection cut 

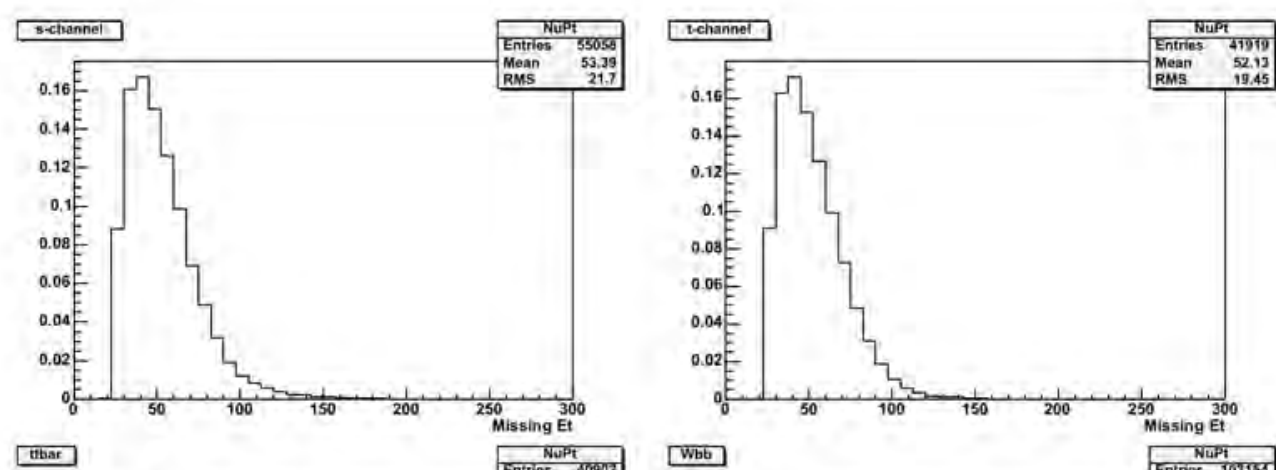

this
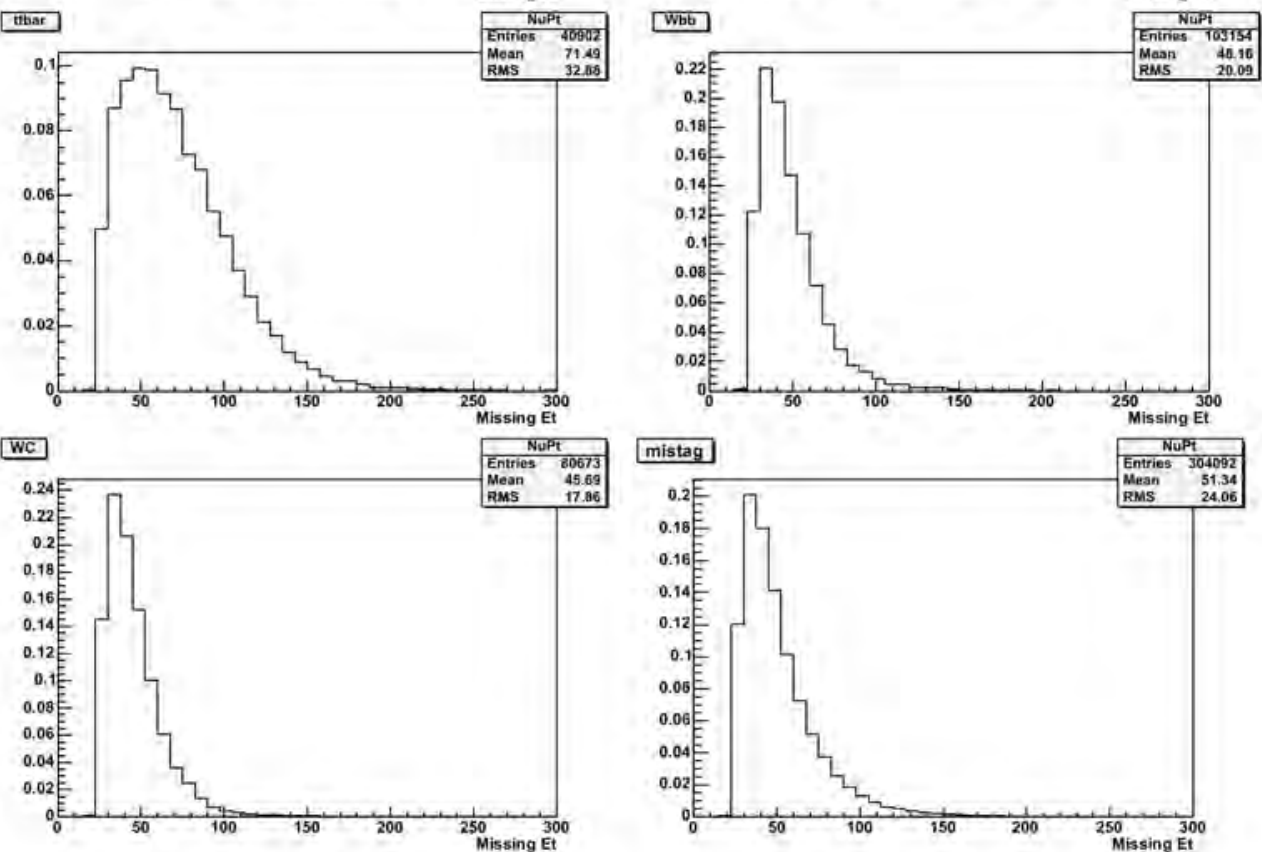

Figure 4.4. Missing Et of s-channel, t-channel, tte, $W \mathbf{b} \overline{\mathbf{b}} \mathbf{W} \mathbf{c}(\overline{\mathbf{c}})$ and mistag after selection cut 

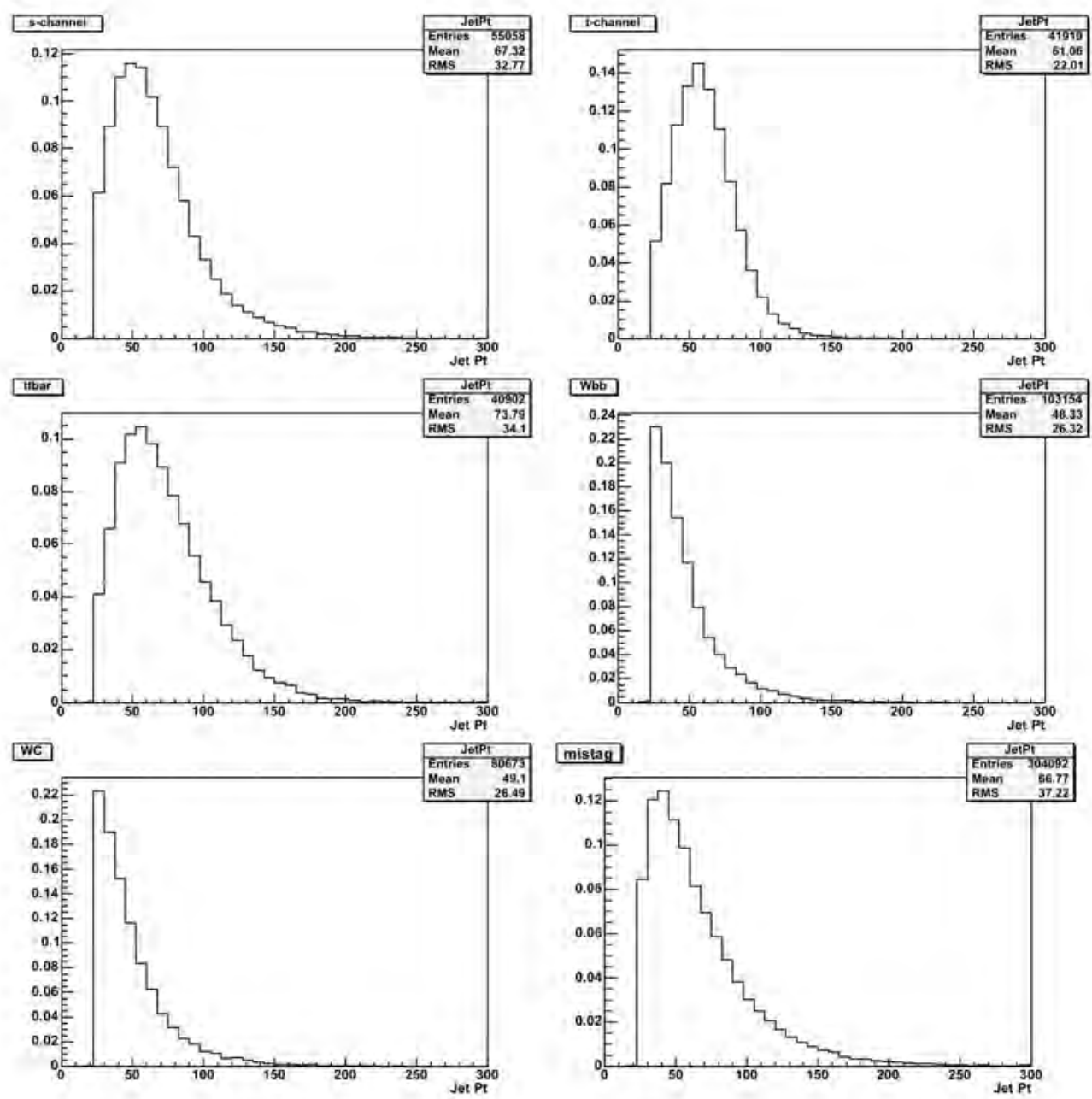

Figure 4.5. Tagged Jet Pt of s-channel, t-channel and $t \bar{t}$ after selection cut 

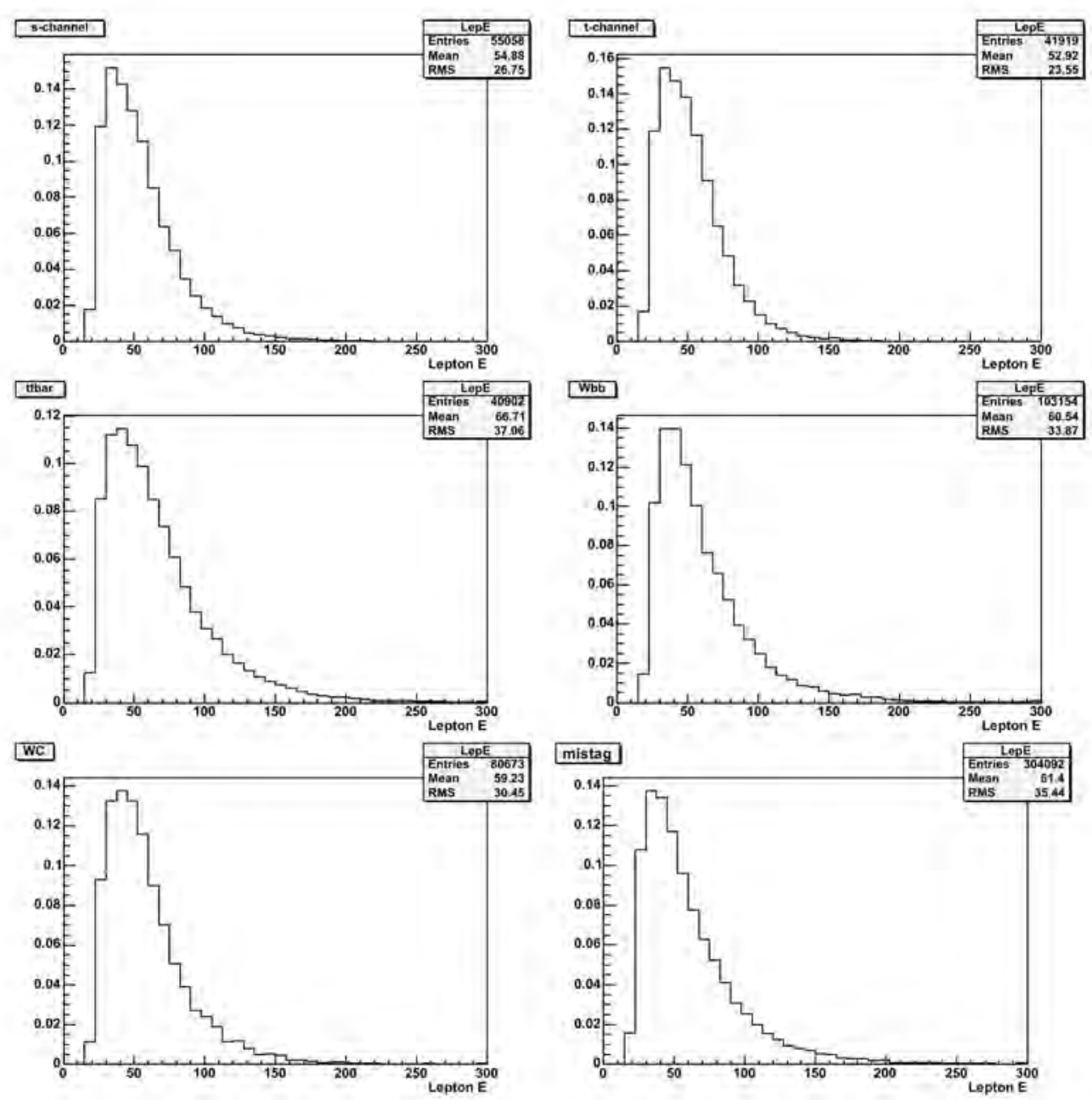

Figure 4.6. Lepton Energy of s-channel, t-channel, tt $\overline{\mathbf{t}}, \mathrm{W} \overrightarrow{\mathbf{b}} \mathbf{\mathbf { b }} \mathrm{Wc}(\overline{\mathbf{c}})$ and mistag after selection cut 

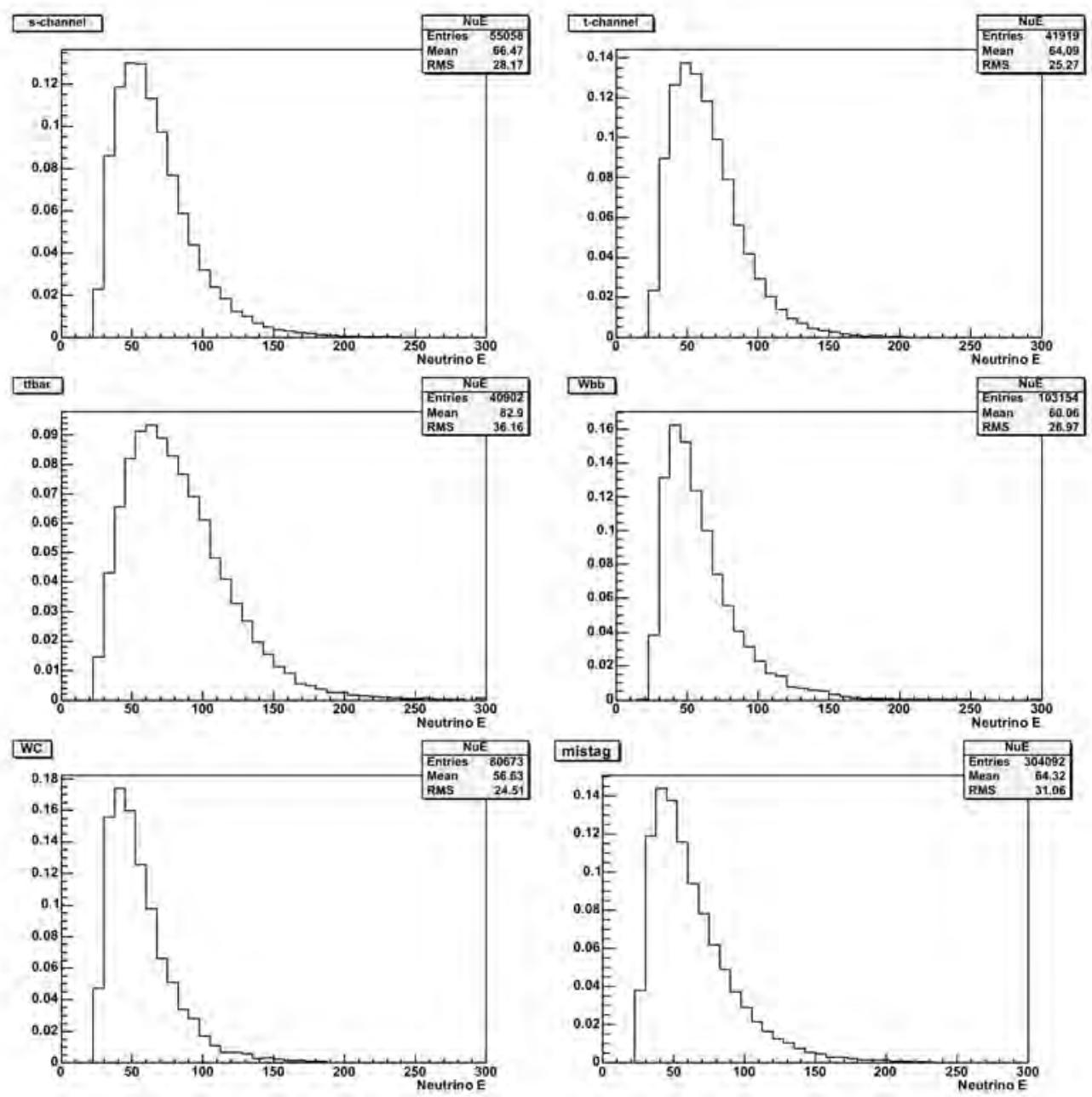

Figure 4.7. Neutrino Energy of s-channel, t-channel, t $\overline{\mathbf{t}}, \mathrm{Wbb} \mathbf{b c}(\overline{\mathbf{c}})$ and mistag after selection cut 

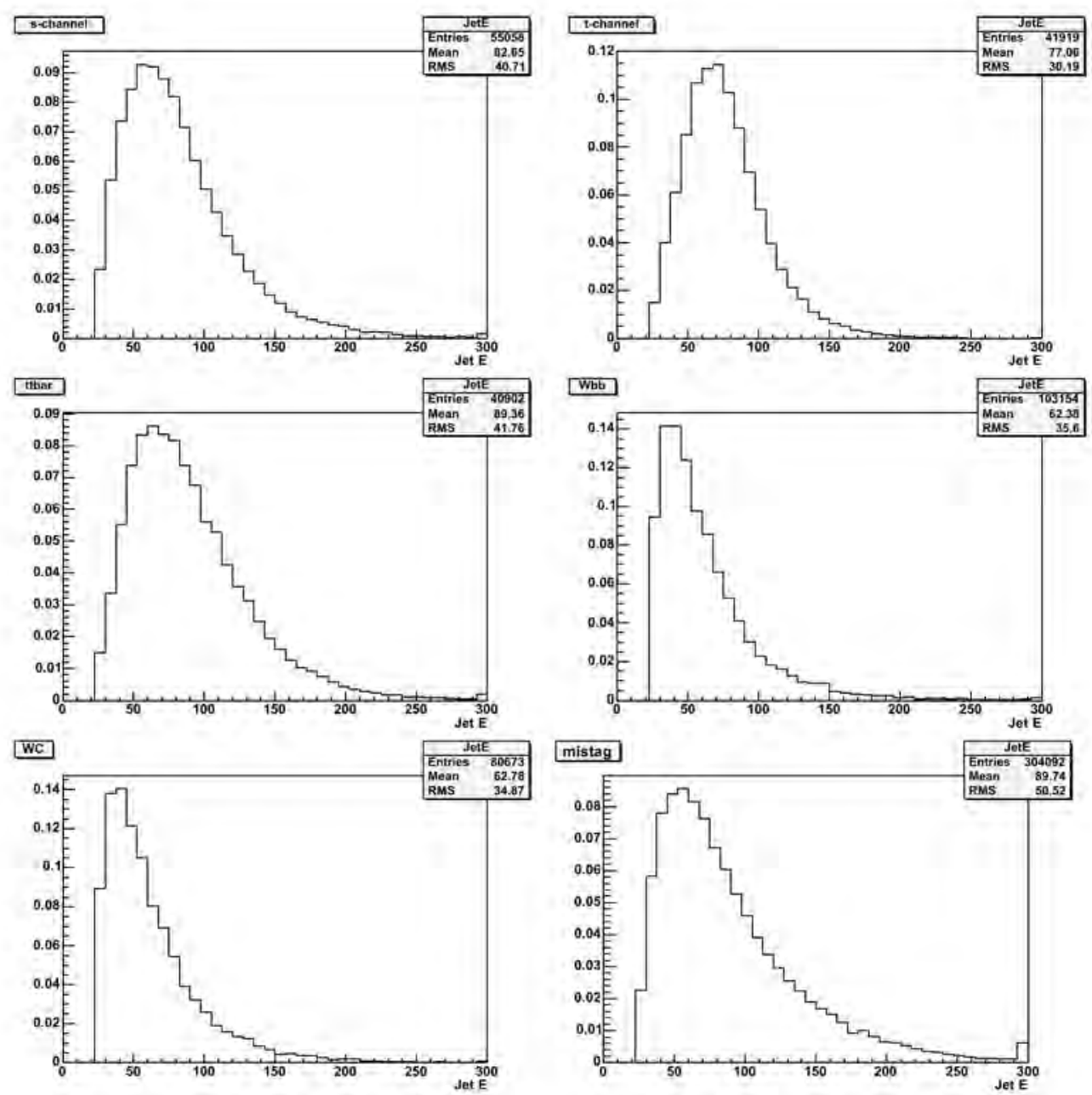

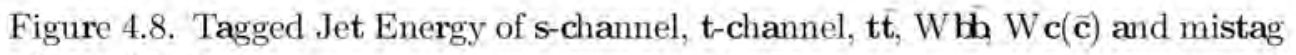
after selection cut 


\section{Chapter 5}

\section{Likelihood Method}

This analysis present the measurement of single-top production using a multivariate likelihood function $(\mathrm{LF})$ technique with $4.8 \mathrm{fb}^{-1}$ data at pp collision at $\mathrm{P} \overline{\mathrm{s}} / 1.96 \mathrm{TeV}$. It is difficult to distinguish signal from the backgrounds due to much background compared to single-top signal events. To observe single-top events, we used a technique of multivariate likelihood function. The discriminant input variables are chosen to separate optimally signal from the background. Many kinematic variables are used for the discriminant variables using four vector of lepton, neutrino, b let or light quark |et. Finally, those input variables are combined into one discriminant(LF).

\subsection{Likelihood Fuction}

To distiguish single-top signal events from backgrounds, we used the multivariate likelihood function discriminant and require at least one b tagged let in 2|et or $3 \mid$ et events.

The single-top final state have one tight lepton, a neutrino, and two or three tight lets. To find four-vectors of the $\mathbf{b}$ let and the neutrino(reltively lepton momentum vector is well measured), the kinematic solver is used by constraing the mass of top quark $(175 \mathrm{GeV})$ and $\mathrm{W}$ boson $(80.4 \mathrm{GeV})$. The output from the kinematic solver is a set of neutrino $\mathrm{Pz}$ and $\mathbf{b}$ - /et four vector and used to reconstruct kinematic variables. We choose the input variables for each 2 et and 3 |et events to maximally separate signal from background.

One of strongest input variables is $\mathrm{Q} \times \eta$, the charge of the lepton times the pseudorapidity of the not b-tagged |et. This gives asymetry for t-channel singletop production because the quark which donates the $\mathrm{W}$ travels predominantly in the same direction it was going before the interaction and the lepton charge determines the sign of the top quark. As another angle-related variable, the 
angle of cosine between the lepton and the untagged let in the top rest frame. The signal(especially t-channel) is distiguished from background because the electroweakly produced top quark is nearly $100 \%$ polarized. For energy related variables, $\mathrm{H}_{T}$, the scalar sum of the transverse energies of the two lets, the lepton, the missing energy, and $\mathrm{M}_{l \nu b}$, reconstructed top mass, and the mass of two lets are considered. The strategy is to combine various variables to optimize the likelihood function. Generally, a large number of variables are considered and choose the best input variables.

The likelihood fuction $\mathrm{L}$ is constructed by forming histograms of each variables. The value of each bin of histograms is denoted $\mathrm{f}_{i j k}$ where $\mathrm{i}$ is the variable, | is the bin, and $\mathrm{k}$ is the event class ( $\mathrm{k} / 1$ for t-channel signal and $\mathrm{k} / 2,3,4,5$ for backgrounds). Because t-channel signal events compared to the s-channel has good features for the separation from backgrounds, the variables is formed for t-channel likelihood function $\mathrm{L}_{t}$. For this fucntion, four background classes are used; $W \bar{b} \overline{\mathbf{b}} \mathbf{t t}, W c \bar{c} / W c$ and mistags. These histograms are normalized to unit area such that $\sum_{j=1}^{n_{i}} \mathrm{f}_{i, j k} / 1$. The likelihood function calculated by evaluating which bin | a given event falls in the distribution of variable i.

$$
p_{i k} / \frac{f_{i j k}}{\sum_{m=1}^{5} f_{i j k}}
$$

This value is used to compute

$$
\mathrm{L}_{s i g} / \frac{\prod_{i=1}^{n_{\text {var }}} p_{i, s i g}}{\sum_{m=1}^{5} \prod_{i=1}^{n_{\text {var }}} p_{i m}} .
$$

The likelihood method dose not consider the correlations between the input variables, but this method is easy to put a number of differnt configurations of the input variables compared to other multivariate techniques at CDF,

In this analysis, two likelihood functions are calculated for two-let events and three-let events; the input variables are chosen separately to optimize the likelihod function for each $2 \mid$ et and $3 \mid$ et events.

\subsection{Input variables}

The input variables for likelihood function are considered by using angular and energy related features of produced events(the lepton, the neutrino(missing transverse energy), $\mathbf{b}$ tagged (et or untagged (et) as well as the MadGraph matrix elements. There are the input variables of $\mathrm{Q} \cdot \eta, \mathrm{M}_{j j}, \cos \theta_{l q}$, KIT Flavor Separator. For only 2 et, $\mathrm{H}_{T}, \log \left(\mathrm{ME}_{t-c h a n}\right), \chi_{t}^{2}$ are added to the input variables. With the presence of a third /et in each event, a larger number of reconstructed 
varaibles is possible. Therefore, more variables are considered when constructing the 3-|et bin likelihood function. For only $3 \mid$ et, $\mathrm{M}_{l \nu b}$, the number of $\mathbf{b}$-tagged lets, the smallest $4 \mathrm{R}$ of any two lets, $\mathrm{P}_{T}$ of the lowest momentum let, $\mathrm{P}_{T}$ of the let chosen to be the $\mathbf{b}$ from top decay, $\eta$ of the reconstruceted $\mathrm{W}$ boson are added to the input variables.

- $\mathrm{Q} \times \eta$, the product of the charge of the lepton and the pseudorapidity of the light quark

This gives the asymetry for t-channel signal events, while it is central for backgrounds. The initial state u-quark in the proton which is composed of two u-quark and a d-quark mostly contributes the single-top production, while d-quark in antiproton gives smaller contribution to that production in t-channel. The light quark which donates the $\mathrm{W}$ boson from the initial valence quark travles predominantly in the same direction of the valence quark before interaction, while the b quark as a sea quark carries small momentum fraction from initial hadron. Therefore, the light quark (ets mostly moves in proton direction, which means positive pseudorapidity, and the lepton charge determines the the sign of the top quark (Figure 5.1, 5.2).
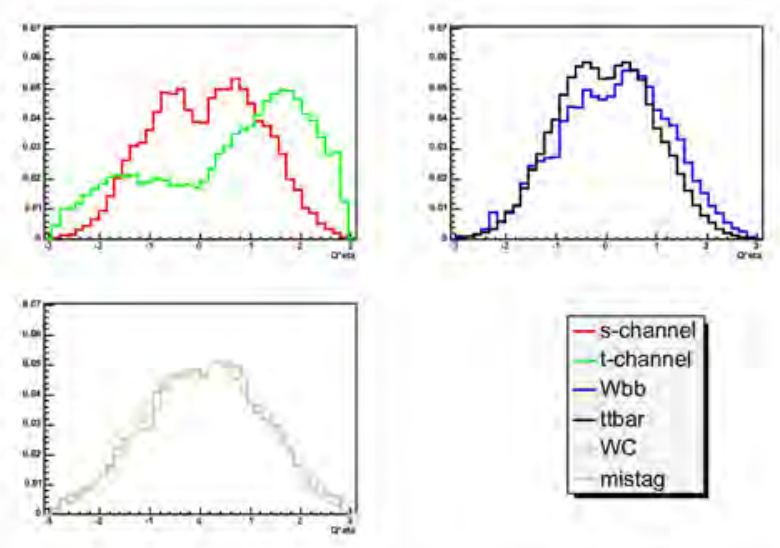

Figure 5.1. Q $\%$ of the lepton charge and untagged |et $\%$ input variable in 2|et bin (normalized plots) 

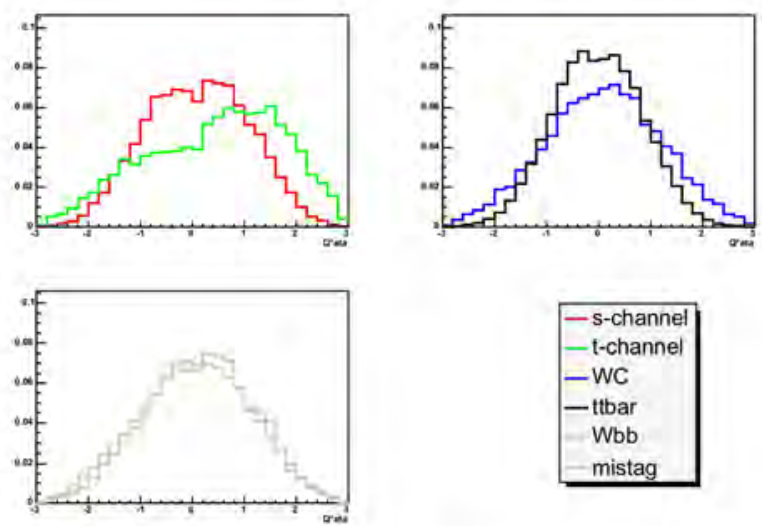

Figure 5.2. Q $\cdot \eta$ of the lepton charge and untagged |et $\eta$. input variable in $3 \mid$ et bin (normalized plots) 
- $\mathrm{M}_{j j}$, the invariant mass of the two lets

For $\mathrm{W}+\mid$ ets events, two lets mainly come from the gluon splitting and the invariant mass is lower than single-top siganl and $t \bar{t}$ events. For the diboson processes, the invariant mass of two lets is expected to be the $\mathrm{W}$ and $Z$ boson mass (Figure $5.3,5.4)$.
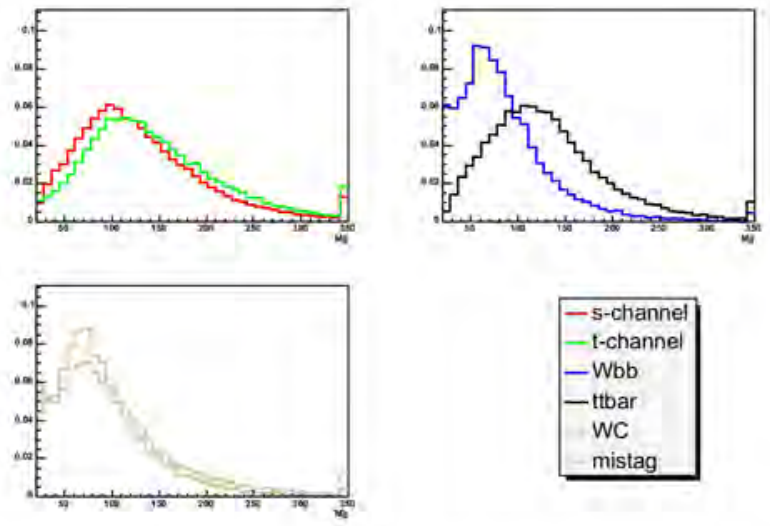

$$
\begin{aligned}
& \text { - s-channel } \\
& \text { - t-channel } \\
& \text { - Wbb } \\
& \text { - ttbar } \\
& \text { WC } \\
& \text { mistag } \\
& \hline
\end{aligned}
$$

Figure 5.3. The invariant mass of the two let. input variable in 2 |et bin (normalized plots)
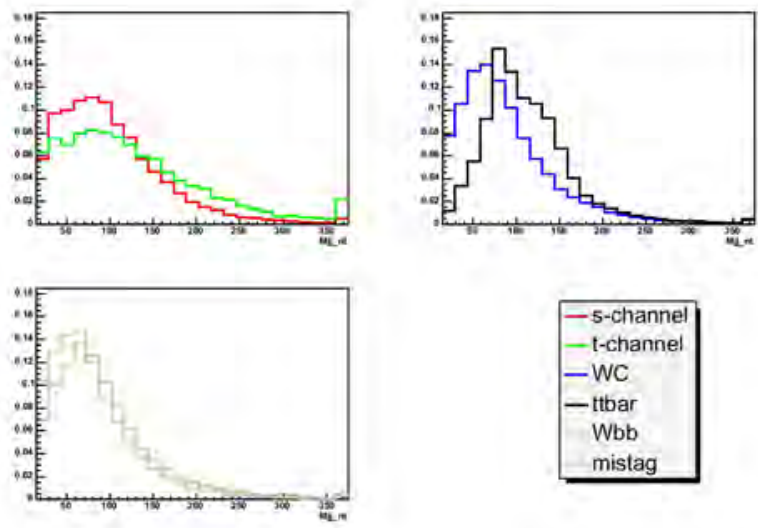

Figure 5.4. The invariant mass of the two let. input variable in 3 let bin (normalized plots) 
- $\cos \theta_{l q}$, the cosine of the angle between the lepton and the light quark(untagged (et) in the top quark rest frame.

The top spin polarized to the direction of light quark momentum in t-channel single-top events. The top quark decays to the $\mathrm{W}$ boson, and $\mathrm{W}$ boson decays to the lepton and neutrino satisfying the angular momentum conservation. The lepton momentum is correlated to the top quark spin(that is light quark momentum) and this variable $(\cos \theta)$ contributes to distiguishing t-channel sample from background (Figure 5.5, 5.6).
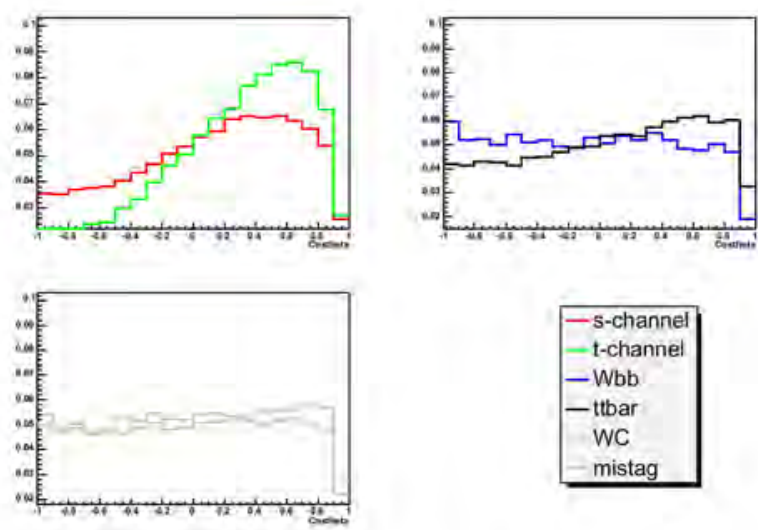

Figure 5.5. $\cos \theta_{l_{q}}$. input variable in $2 \mid$ et bin (normalized plots)
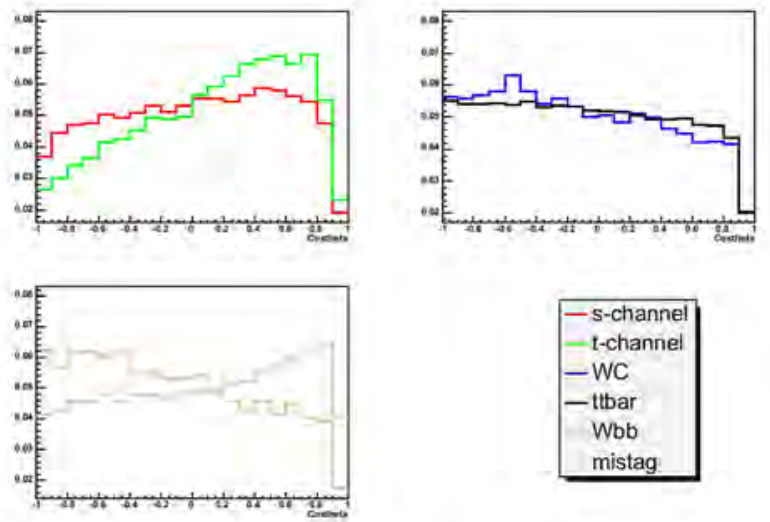

$$
\begin{array}{r}
\text { - } 5 \text {-channe } \\
\text { - t-channel } \\
\text { - WC } \\
\text { - ttbar } \\
\text { Wbb } \\
\text { mistag } \\
\hline
\end{array}
$$

Figure 5.6. $\cos \theta_{l q}$. input variable in $3 \mid$ et bin (normalized plots) 
- KIT Flavor Separator, the output of Neural Network for the choice of bquark let.

The range of output of NN btagger is from -1 to 1 . The outputs of signal, $t \bar{t}$ or $\mathbf{W b}$ events containing b-quark go to the +1 and the ouputs of $\mathrm{Wc}(\mathbf{c})$ and mistags go to the -1 (Figure $5.7,5.8$ ).
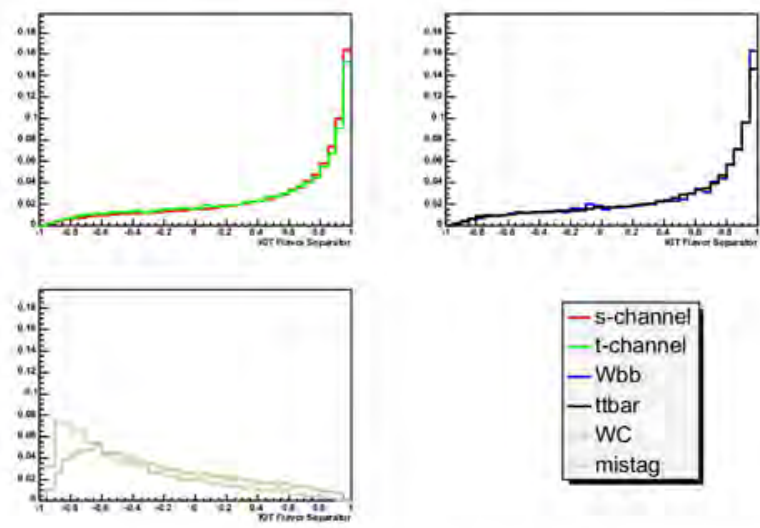

Figure 5.7. NN-btagger. input variable in $2 \mid$ et bin (normalized plots)
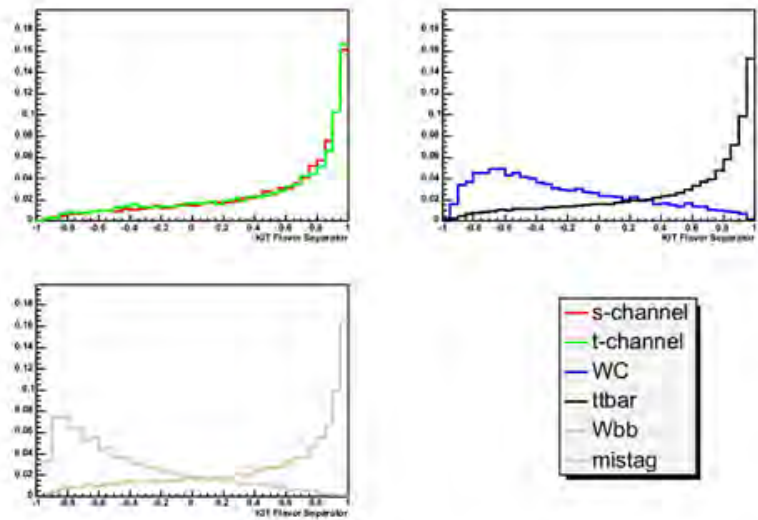

Figure 5.8. NN-btagger. input variable in 3 et bin (normalized plots) 
- $\mathrm{H}_{T}$, the scalar sum of the transverse energies of the lepton, and the missing transverse energy, and the two highest momentum |ets.

The peak of signal events is lower than $t \bar{t}$ events and higher than other backgrouns (Figure 5.9).
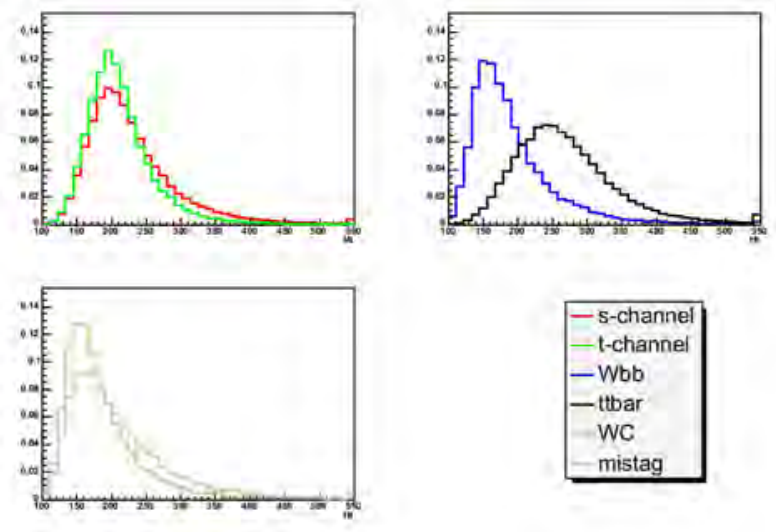

Figure 5.9. $\mathrm{H}_{T}$. The scalar sum of transverse energies of the two lets, the lepton, and the missing transeverse energy. input variable in 2 |et bin (normalized plots)

- $\log \left(\mathrm{ME}_{t-\text { chan }}\right)$, is the $\log$ of the MadGraph matrix element

The MadGraph matrix element is computed using the constrained four-vectors of the b-quark, the lepton and the neutrino, assuming t-channel signal production. The matrix element was a weighted average of the $\mathrm{ub} \rightarrow \mathrm{dt}$ matrix element with weight $2 / 3$, and the same matrix element with lepton sign reversed with weight $1 / 3$ in order to simulate $d \bar{b} \rightarrow u \bar{t}$. This increases the signal-like behavior of the t-channel signal, but also increases the background prediction. The overall sensitivity of the analysis was improved and this can be understood as the optimization of the search for the larger subclass of the signal (Figure 5.10).

- $\chi_{t}^{2}$, the output from the kinematic solver for the t-channel selection algorithms

The kinematic constraint is the top-quark mass $\left(M_{l \nu b} / M_{t}\right)$ and $W$ boson $\operatorname{mass}\left(\mathrm{M}_{l \nu} / \mathrm{M}_{W}\right)$. This constraint allows a solution for the b-let's energy and improve the reconstruction of signal events, worsen the reconstruction of background events. Therefore, the kinematic solver helps to separate the signal events from the backgrounds (Figure 5.11). 

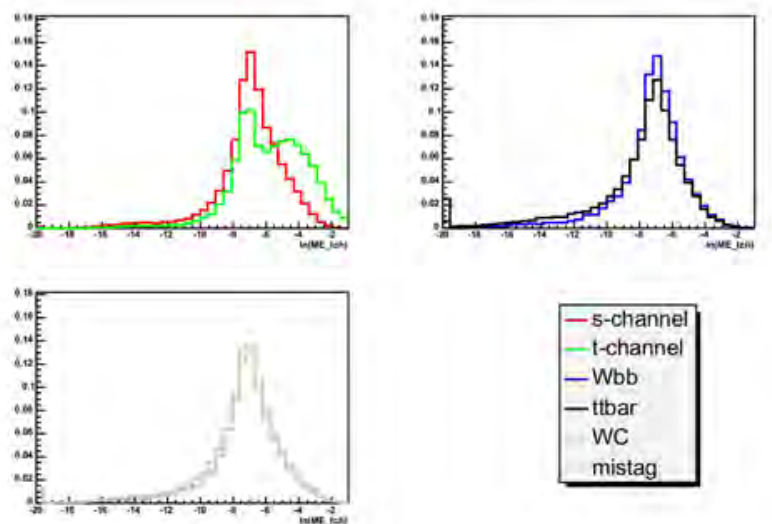

Figure 5.10. Matrix Element assuming t-channel signal production. input variable in 2 et bin (normalized plots)
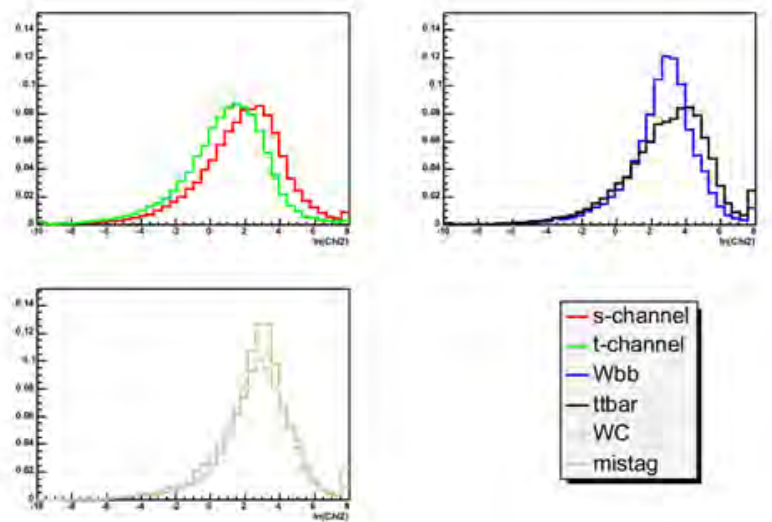

Figure 5.11. $\chi^{2}$ output from the kinematic solver. input variable in 2 et bin (normalized plots) 
- $\mathrm{M}_{l \nu b}$, the reconstructed top-quark mass of lepton, neutrino, and b-quark let The invariant mass peak(around $170 \mathrm{GeV}$ ) of signal events is higher value than it of backgrounds events (Figure 5.12).
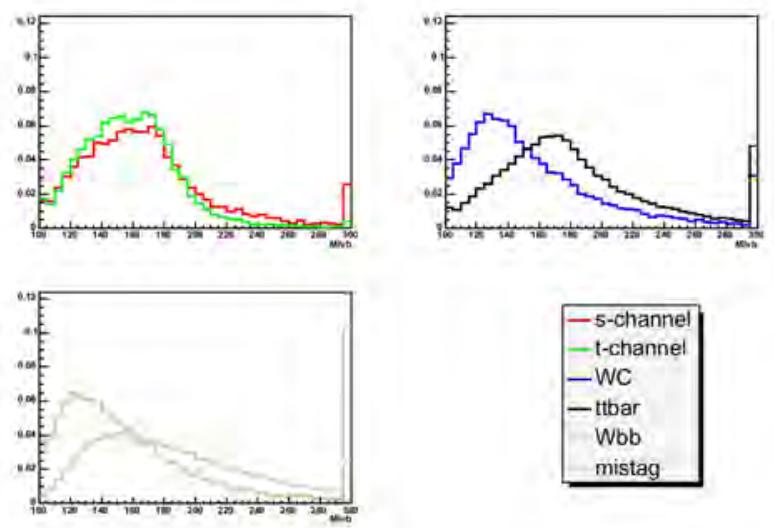

$$
\begin{gathered}
\text {-5-channel } \\
\text { - t-channel } \\
\text { - WC } \\
\text { - ttbar } \\
\text { Wbb } \\
\text { mistag } \\
\hline
\end{gathered}
$$

Figure 5.12. Reconstructed top mass. input variable in 3 et bin (normalized plots)

- The number of b-tagged lets

This variable can differentiate sample of small $\mathbf{b}$ /et from larger number of $\mathbf{b}$ /et samples (Figure 5.13).
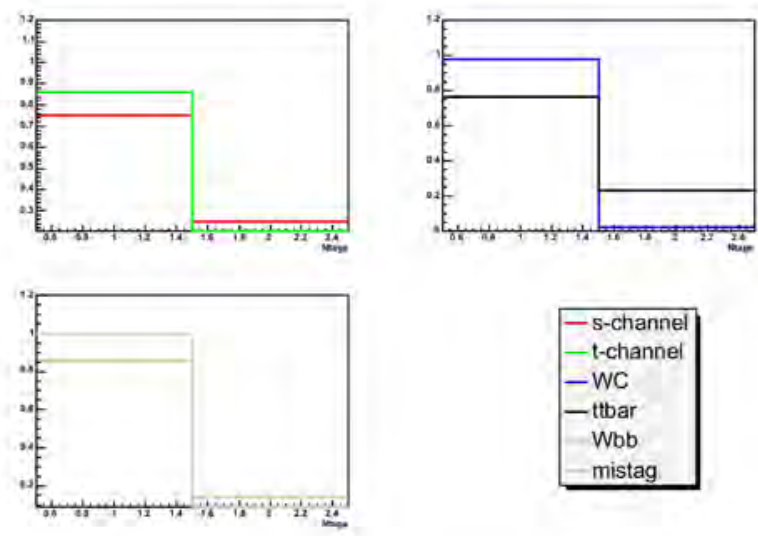

$$
\begin{aligned}
& \text { - s-channel } \\
& \text { - t-channel } \\
& - \text { WC } \\
& \text { - ttbar } \\
& \text { Wbb } \\
& \text { mistag }
\end{aligned}
$$

Figure 5.13. The number of $\mathbf{b}$-tagged |ets. input variable in 3 et bin (nornalized plots) 
- The smallest $4 \mathrm{R}$ of any two lets ; This is a discriminant variable from the properties of relations between two lets. For example, the angle of two lets came from gluon is different from the angle of other pair of |ets (Figure 5.14).
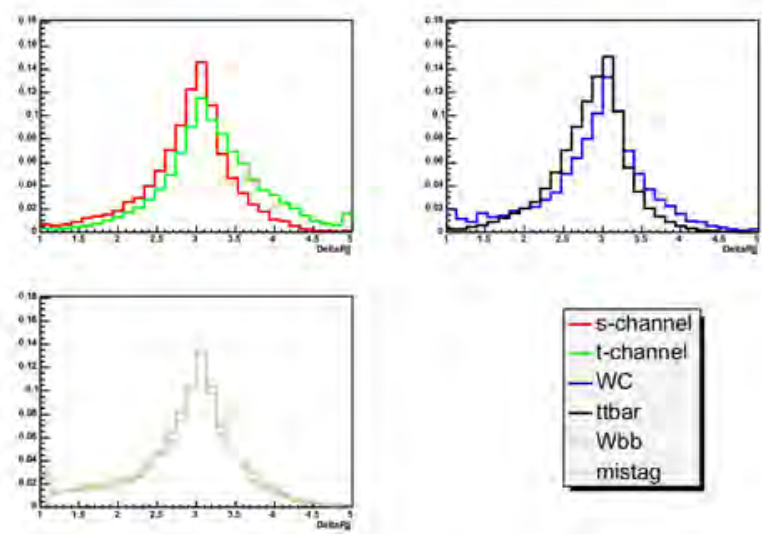

Figure 5.14. The smallest $4 \mathrm{R}$ of any two let. input variable in 3 |et bin (normalized plots)

- $\mathrm{P}_{T}$ of the lowest momentum let ; The backgrounds such as Wb W $\mathbf{c}(\mathbf{c})$ and mistag have lower momentum distribution than signal and $t \bar{t}$ (Figure 5.15).
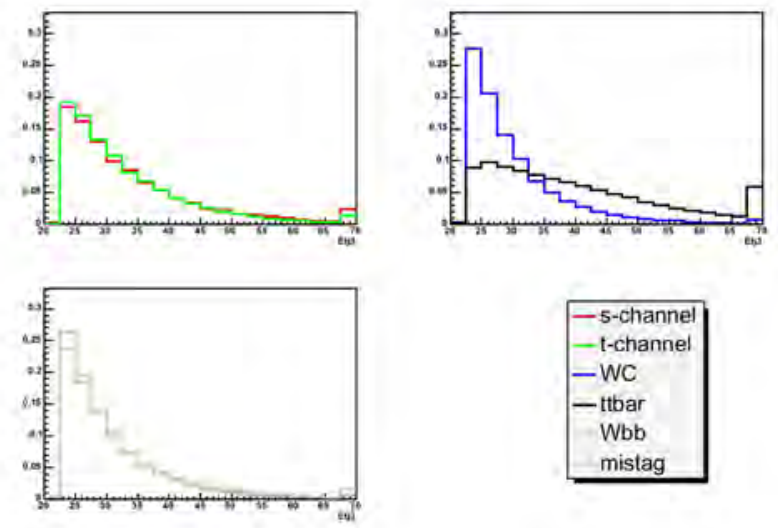

Figure 5.15. $\mathrm{P}_{t}$ of the lowest momentum |et. input variable in $3 \mid$ et bin (normalized plots) 
- $\mathrm{P}_{T}$ of the let chosen to be the $\mathbf{b}$ from top decay ; This is also similar situation with previous varaible (Figure 5.16).
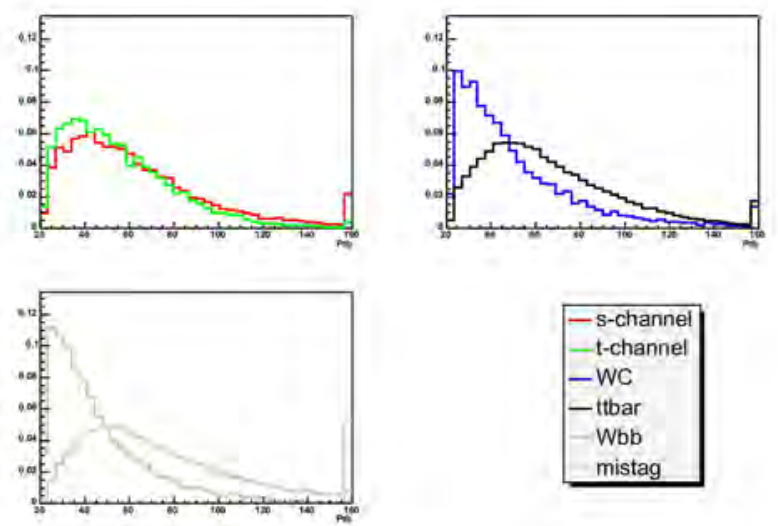

Figure 5.16. $\mathrm{P}_{t}$ of the et chosen to be the $\mathbf{b}$ from top decay. input variable in 3 et bin (normalized plots)

- $\eta$ of the reconstruceted W boson (Figure 5.17).
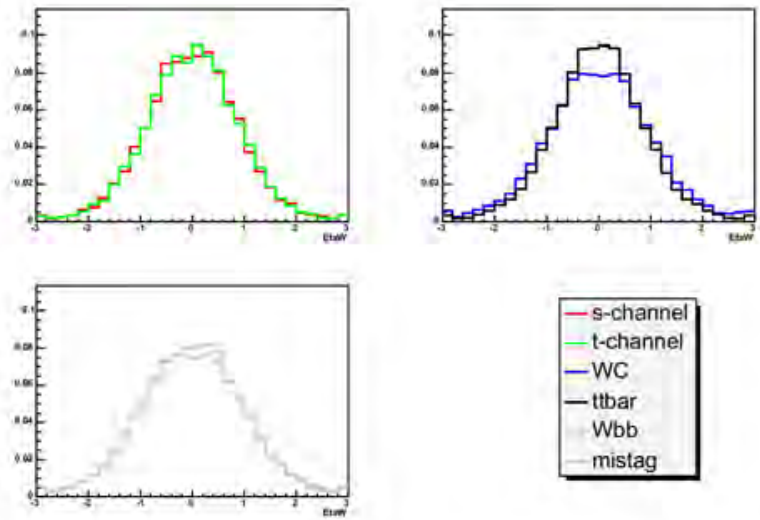

$$
\begin{aligned}
& \text { - s-channel } \\
& \text { - t-channel } \\
& \text { - WC } \\
& \text { - ttbar } \\
& \text { Wbb } \\
& \text { mistag }
\end{aligned}
$$

Figure 5.17. $\eta$ of W. input variable in 3|et bin (normalized plots)

It is difficult to distinguish signal from background by using only one input variable. So, we combined proper input variables, they are already mentioned, to one likelihood function discriminant. Figure 5.18 5.24 show the stacked plots(Data and MC are compared) of input variables. 


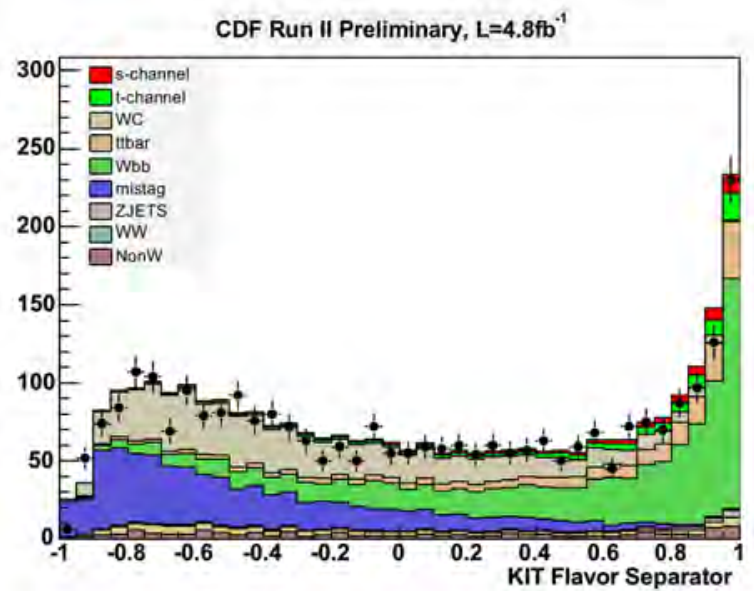

Figure 5.18. NN-btagger. input variable in 2|et bin (Data and MC are compared)

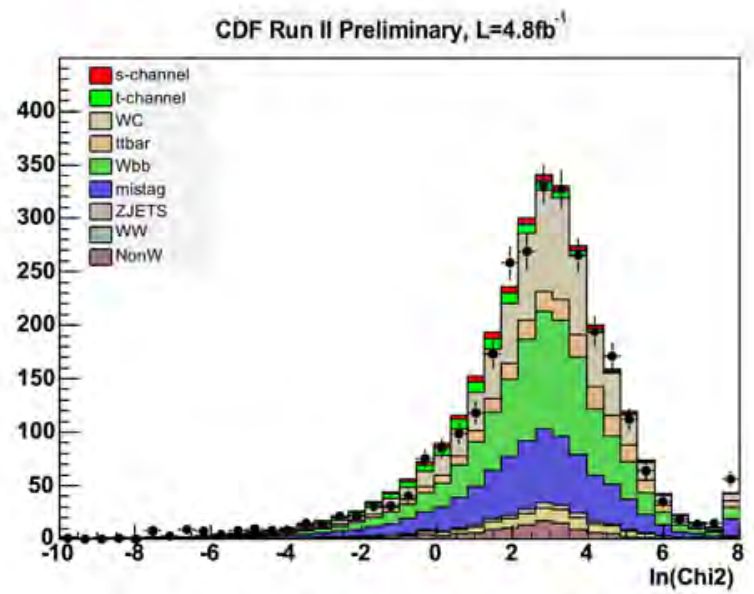

Figure 5.19. $\chi^{2}$ output from the kinematic solver. input variable in 2/et bin (Data and $\mathrm{MC}$ are compared) 


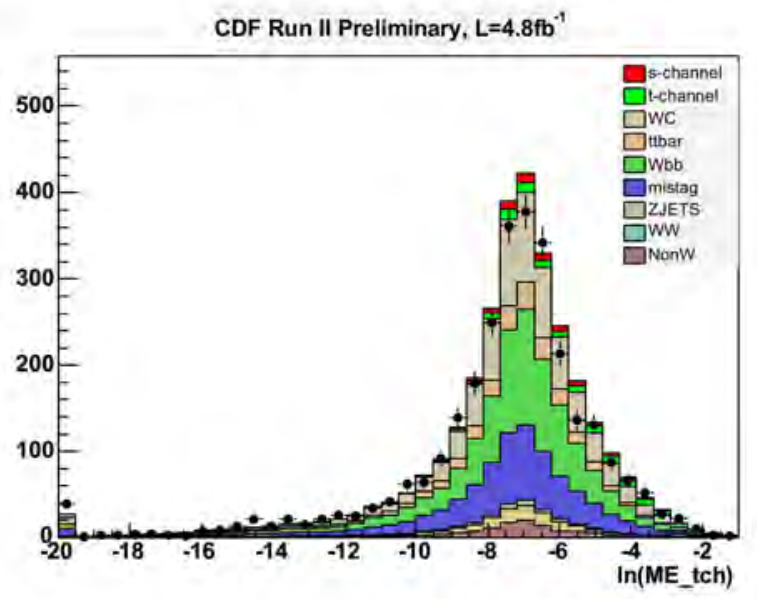

Figure 5.20. $\log \left(\mathrm{ME}_{t-c h}\right)$. Output from the kinematic solver. input variable in 2 et bin (Data and MC are compared)

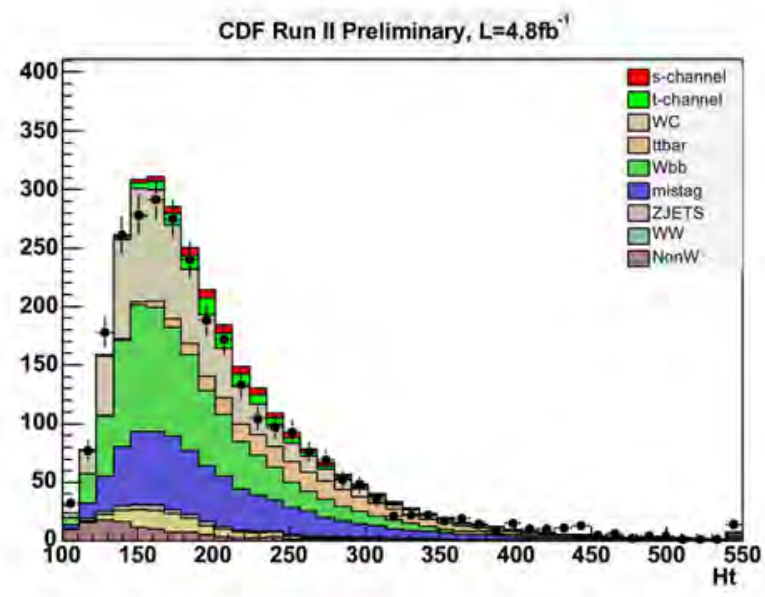

Figure 5.21. $\mathrm{H}_{T}$. The scalar sum of transverse energies of the two /ets, the lepton, and the missing transeverse energy, input variable in 2/et bin (Data and MC are compared) 


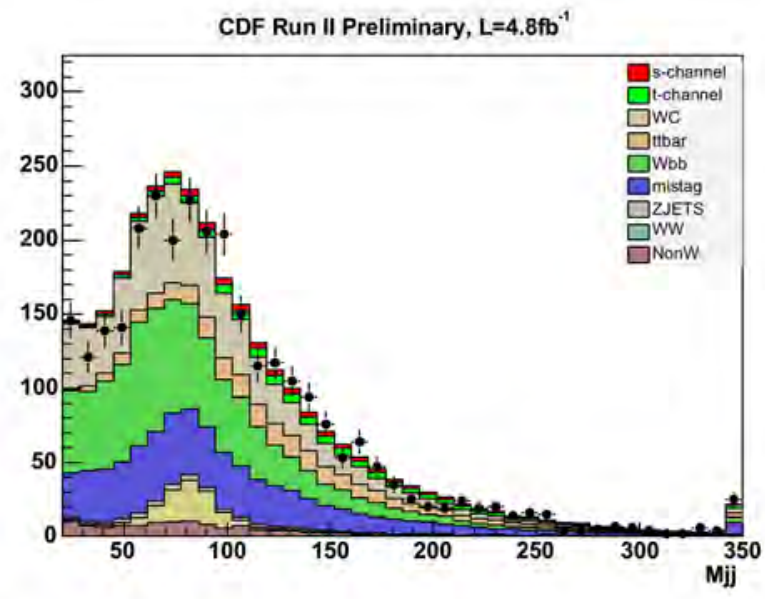

Figure 5.22. The invariant mass of the two et. input variable in 2|et bin (Data and $\mathrm{MC}$ are compared)

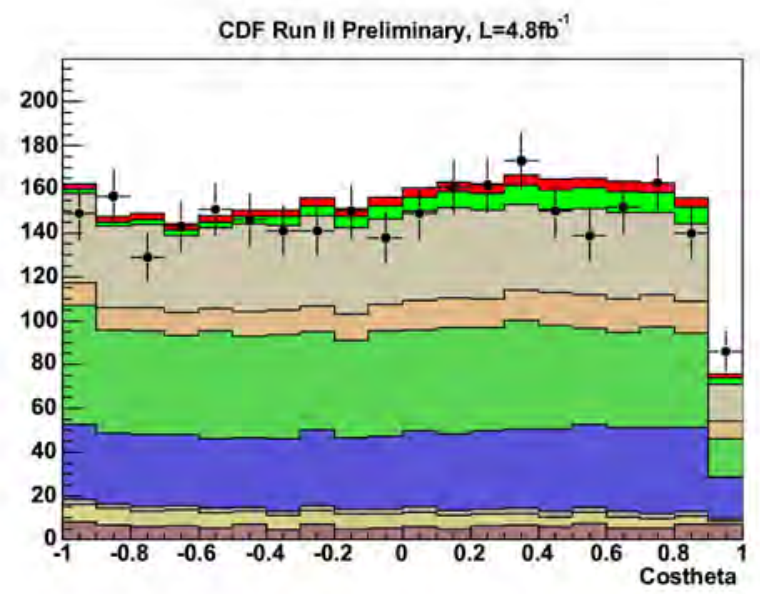

Figure 5.23. $\cos 0_{l q}$. input variable in $2 \mid$ et bin (Data and $\mathrm{MC}$ are compared) 


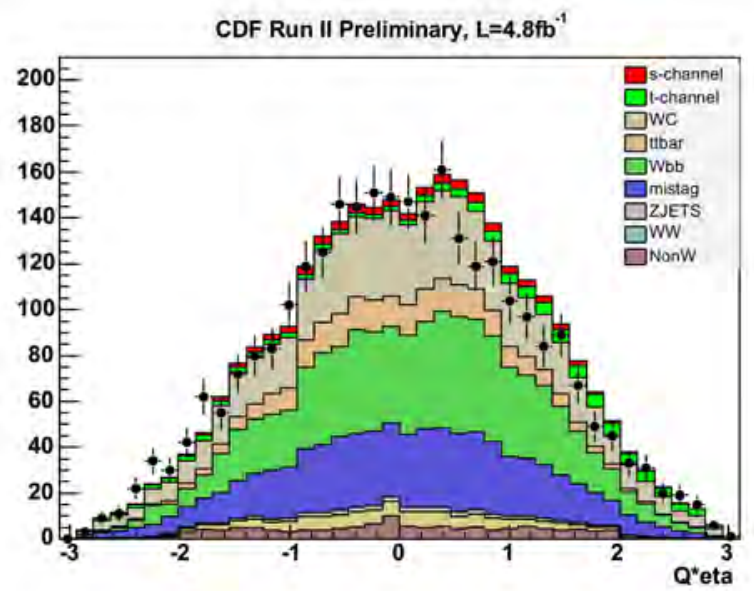

Figure 5.24. Q $\cdot \eta$ of thelepton charge and untagged |et $\eta$. input variable in $2 \mid$ et bin (Data and $\mathrm{MC}$ are compared)

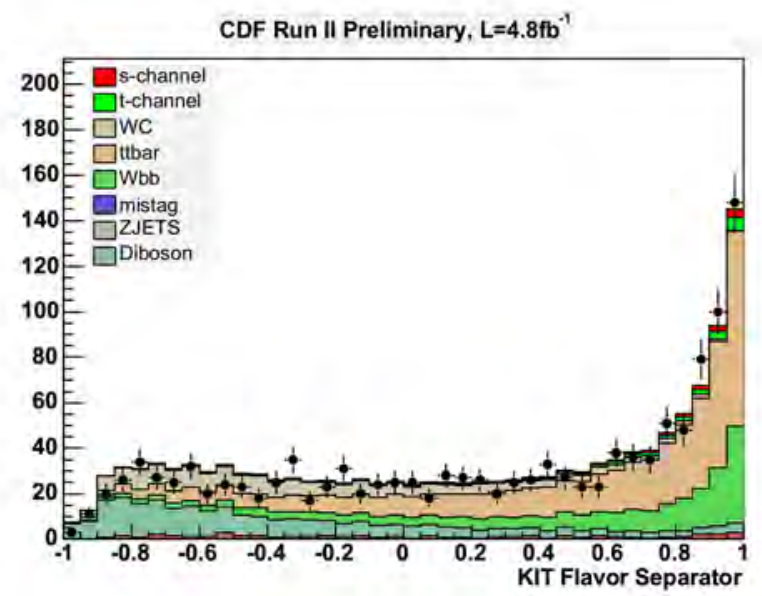

Figure 5.25. NN-btagger. input variable in 3 et bin (Data and $\mathrm{MC}$ are compared) 


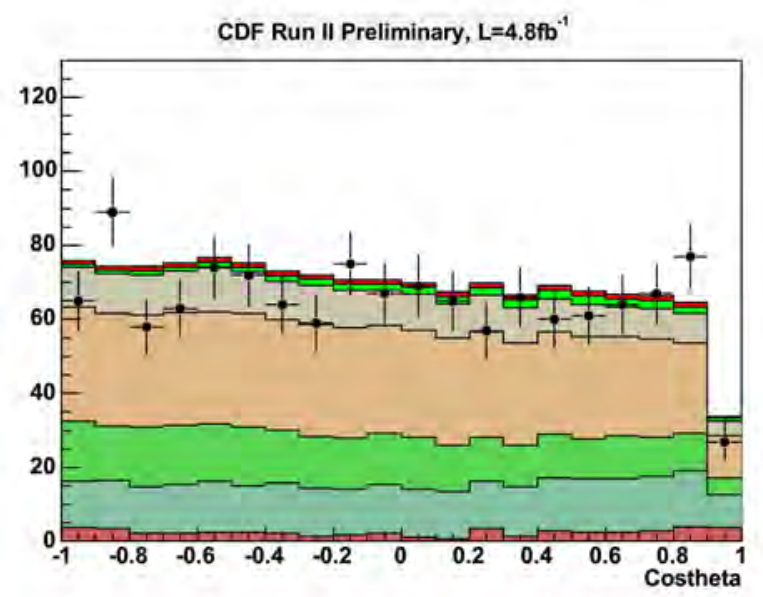

Figure 5.26. $\cos \theta_{l q}$. input variable in $3 \mid$ et bin (Data and MC are compared)

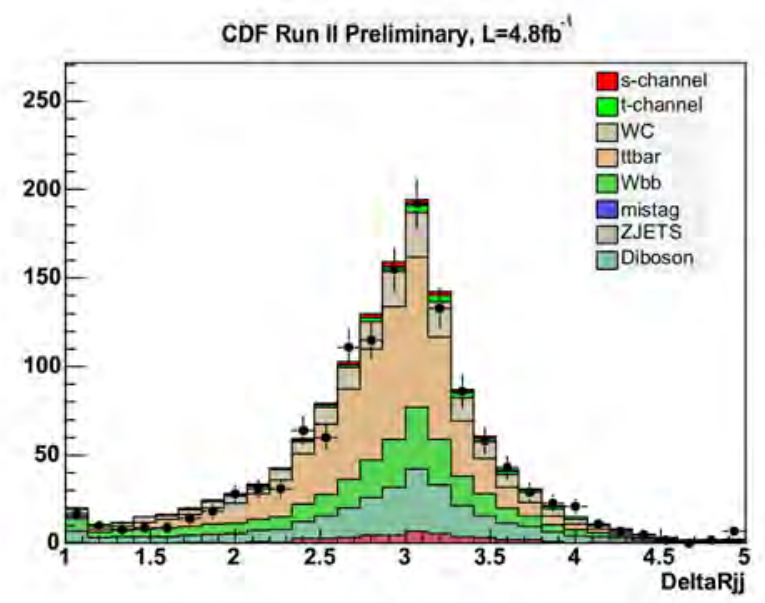

Figure 5.27. The smallest $4 \mathrm{R}$ of any two |et. input variable in 3|et bin (Data and $\mathrm{MC}$ are compared) 


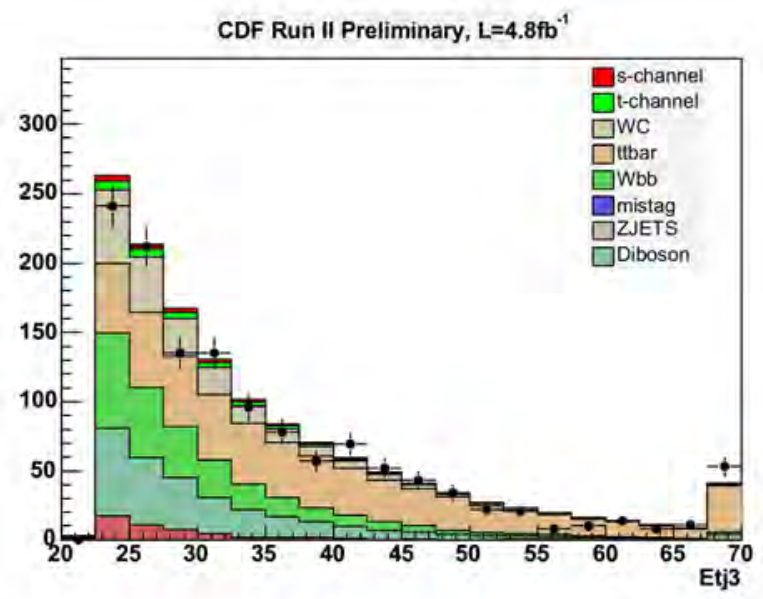

Figure 5.28. Lowest Et of |et. input variable in 3|et bin (Data and MC are compared)

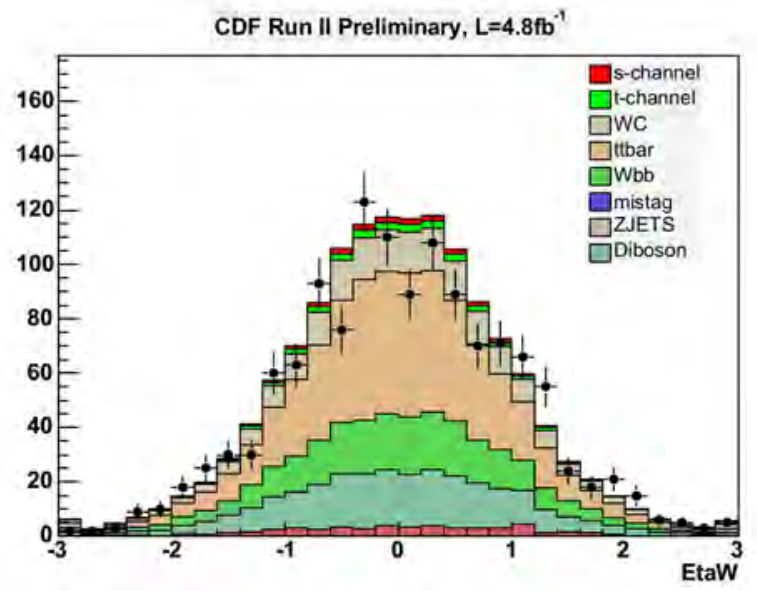

Figure 5.29. $\eta$ of W. input variable in 3 et bin (Data and MC are compared) 


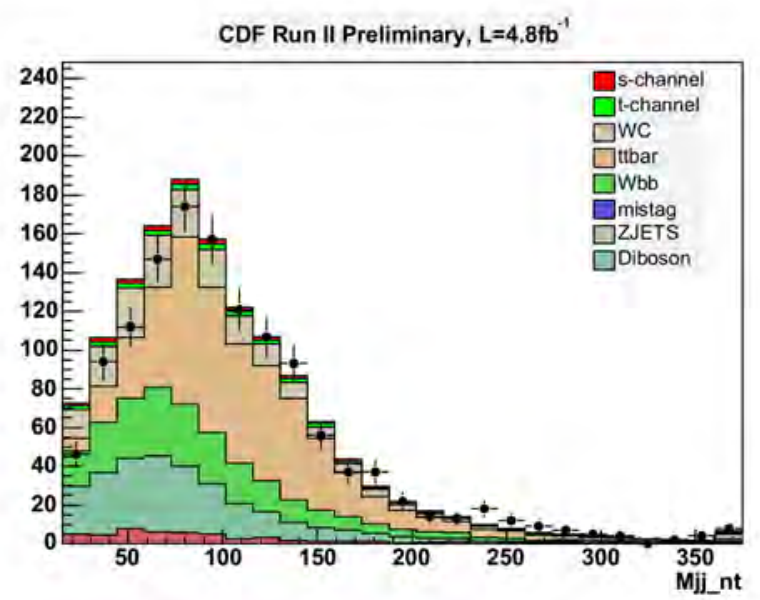

Figure 5.30. Two lets not chosen to be the $\mathbf{b}$ from top. input variable in 3 |et bin (Data and MC are compared)

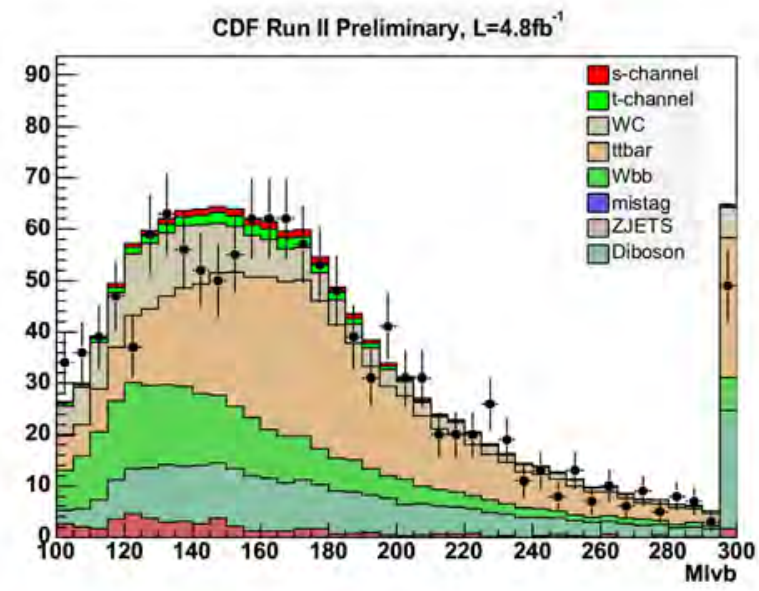

Figure 5.31. Reconstructed top mass. input variable in 3|et bin (Data and MC are compared) 


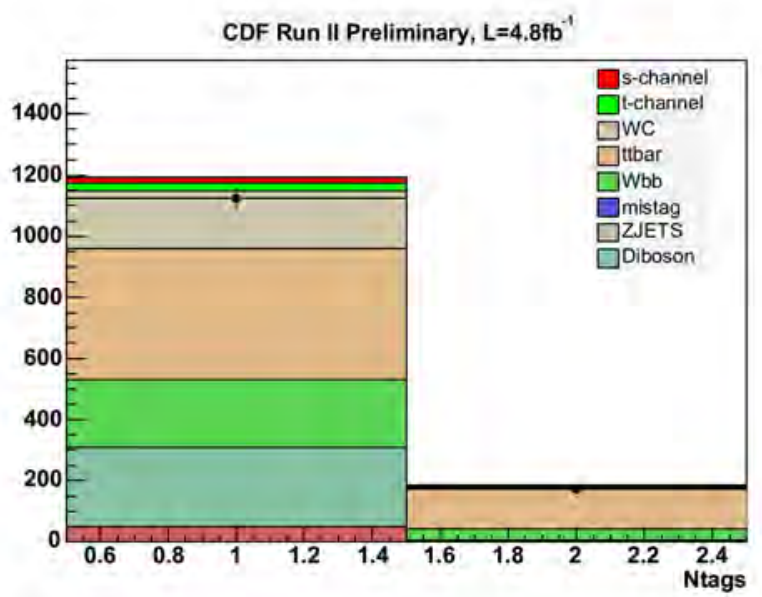

Figure 5.32. The number of $\mathbf{b}$-tagged |ets. input variable in 3 |et bin (Data and MC are compared)

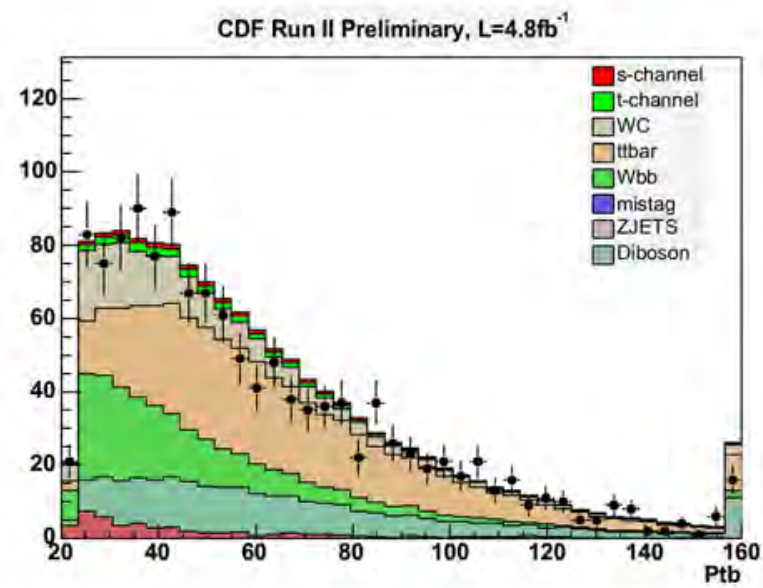

Figure 5.33. $\mathrm{P}_{t}$ of the lowest momentum let. input variable in 3|et bin (Data and $\mathrm{MC}$ are compared) 


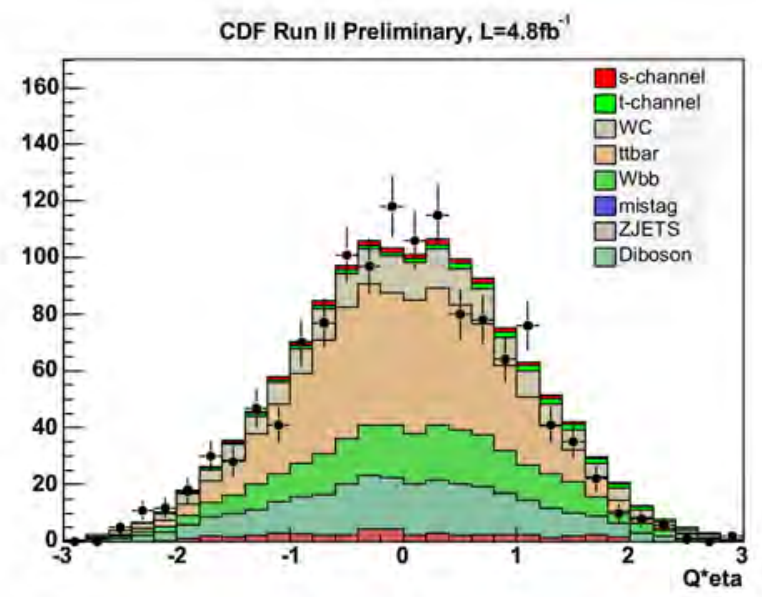

Figure 5.34. Q $\cdot \eta$ of thelepton charge and untagged |et $\eta$. input variable in $3 \mid$ et bin (Data and MC are compared) 


\subsection{Likelihood Function}

The likelihood functions created from input variables are used to measure the single-top cross-section. Figure [5.35], [5.36] show the likelihood function in 2/et and 3/et bin for comparison of MC and data. The right plots are the events of $\mathrm{L}>0.8$. For 2 et events of the region $\mathrm{L}>0.8$, single-top is $41 \%$, Wbb is $29 \%$, $\mathrm{Wcc} / \mathrm{Wc}$ is $19 \%$ and other backgrounds(nonW+Diboson $+Z \mid$ ets $+\mathrm{tt}$ ) are $11 \%$. For 3 et events of the region $\mathrm{L}>0.8$, single-top is $19 \%$, Wbb is $28 \%$, Wcc/Wc is $9 \%$, $\mathrm{t} \overline{\mathrm{t}}$ is $31 \%$ and other backgrounds(nonW+Diboson $+Z \mid$ ets) are $13 \%$. Therefore, we can see that single-top signal has more portion in the high likelihood bin about $40 \%$ for $2 \mid$ et and about $20 \%$ for $3 \mid$ et events.
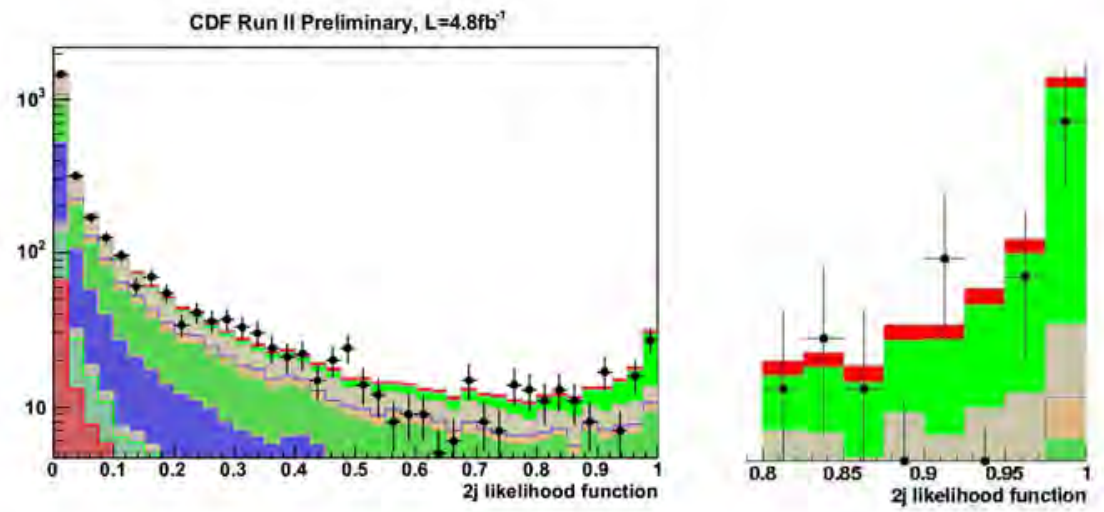

Figure 5.35. 2|et bin t-channel Likelihood function shown on log scale(left plot) and Likelihood function for $\mathrm{L}>0.8$ (left plot) 

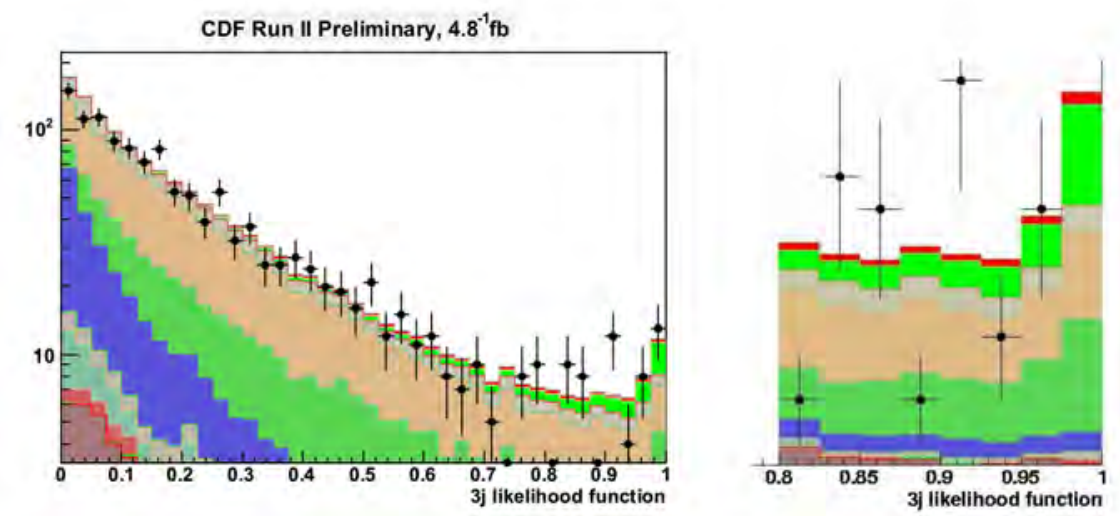

Figure 5.36. 3|et bin t-channel Likelihood function shown on log scale(right plot) and Likelihood function for $\mathrm{L}>0.8$ (left plot) 


\section{Chapter 6}

\section{Extraction of Signal}

The likelihood distiribution(at previous chapter) combined various input templates is applied to the fit code. For fitting the cross section, Maximumlikelihood technique are used. The fitted value is the fraction of the measured cross section in the predicted cross section. The two dimentional fit is done for testing Wtb coupling.

For the degree of evidende of single-top production, p-value is calculated. From this p-value, we defined the evidence $(3 \sigma)$ or observed events $(5 \sigma)$. In the single-top group of CDF, we performed the observaion of single-top production at $3.2 \mathrm{f} \mathrm{b}^{-1}$ at $5 \sigma$ level.

\subsection{Maximum Likelihood Fit}

To measure the cross-section of single-top production, a binned likelihood fit to the multivariate likelihood is performed using a Baysian technique [16] [47].

Given $\mathrm{N}$ channels (from histogram bins or number of $\mid$ ets..), and $\mathrm{n}_{k}$ observed events in the kth channel, $\mathrm{k} / 1,2, \ldots, \mathrm{N}$, the Poisson Probability of obtaining the observed result is

$$
\prod_{k=1}^{N} \frac{\mathrm{e}^{-\left(s \epsilon_{k}+b_{k}\right)}\left(s \epsilon_{k}+\mathbf{b}_{k}\right)^{n_{k}}}{\mathrm{n}_{k} \rightarrow}
$$

where $\mathbf{s}$ represents the parameter of interest (the cross section) and $\epsilon_{k}$ and $\mathbf{b}_{\text {. }}$ are the acceptance and expected background for the kth channel, respectively. All the $\epsilon_{k}$ and $\mathbf{b}_{k}$. have uncertainties and are considered "nuisance parameters".

The loint nuisance prior is written as

$$
\pi(\mathbf{s}) \iiint_{(2 N)} \pi\left(\epsilon_{1} ; \mathbf{b}_{1} ; \cdots ; \epsilon_{N} ; \mathbf{b}_{N}\right)\left[\prod_{k=1}^{N} \frac{\mathrm{e}^{-\left(s \epsilon_{k}+b_{k}\right)}\left(s \epsilon_{k}+\mathbf{b}_{k}\right)^{n_{k}}}{\mathrm{n}_{k} \rightarrow}\right] d \epsilon_{1} \mathrm{~d} \mathbf{b}_{1} \cdots d \epsilon_{N} \mathrm{~d} \mathbf{b}_{\mathrm{N}}
$$


where $2 \mathrm{~N}$ marginalization integrals are performed, and $\pi(\mathrm{s})$, the prior for $\mathbf{s}$, is assumed to be independent of the oint nuisance prior. The random $M$ $\left(\epsilon_{1} ; \mathbf{b}_{1} ; \cdots ; \epsilon_{N} ; \mathbf{b}_{\mathrm{N}}\right)$ vectors are generated.

The marginalized posterior for $\mathrm{s}$ then becomes

$$
\mathrm{p}(\mathbf{s}) / \frac{1}{\mathrm{~N}} \frac{\pi(\mathbf{s})}{\mathrm{M}} \sum_{i=1}^{M}\left[\prod_{k=1}^{N} \frac{\mathrm{e}^{-\left(s \epsilon_{k}+b_{k}\right)}\left(s \epsilon_{k}+\mathbf{b}_{k}\right)^{n_{k}}}{\mathrm{n}_{k} \rightarrow}\right]
$$

where the normalization constant $\mathrm{N}$ is given by

$$
\mathrm{N} / \int_{0}^{1} \frac{\pi(\mathbf{s})}{\mathrm{M}} \sum_{i=1}^{M}\left[\prod_{k=1}^{N} \frac{\mathrm{e}^{-\left(s \epsilon_{k}+b_{k}\right)}\left(s \epsilon_{k}+\mathbf{b}_{k}\right)^{n_{k}}}{\mathrm{n}_{k} \rightarrow}\right] \mathrm{d} \mathbf{s}
$$

In this analysis, the following likelihood function is used.

$$
\mathrm{L} / \prod_{k=1}^{n \text { bins }} \frac{\mathrm{e}^{-\mu_{k}} \mu_{k}^{n_{k}}}{\mathrm{n}_{k} \rightarrow} \cdot \prod_{j}^{b g s} \mathrm{G}_{j}\left(\beta_{j} \mid 4_{j}\right) \cdot \prod_{i}^{s y s} \mathrm{G}_{i}\left(\delta_{i}\right)
$$

where $\mu_{k}$ is given by,

$$
\begin{array}{r}
\mu_{k} / \sum_{j}^{s i g \& b g} \beta_{k} \cdot \sum_{i}^{s y s}\left[1+\left|\delta_{i}\right| \cdot\left(\epsilon_{j i+} \mathrm{H}\left(\delta_{i}\right)+\epsilon_{j i-} \mathrm{H}\left(-\delta_{i}\right)\right)\right] \\
\cdot \alpha_{j k} \cdot \sum_{i}^{s y s}\left[1+\left|\delta_{i}\right| \cdot\left(\mathrm{k}_{j i k+} \mathrm{H}\left(\delta_{i}\right)+\mathrm{k}_{j i k-} \mathrm{H}\left(-\delta_{i}\right)\right)\right]
\end{array}
$$

The fitted parameter is $\beta_{j} / \sigma_{j}^{f i t} / \sigma_{j}^{S M}$, ratio of measured cross-section and SM prediction. The shape information is given by $\alpha_{j k}$ in template bin $\mid$ of process $\mathrm{k}$. The relative strength of a systematic effect for source $i$ is $\delta_{i}$. The $\epsilon_{j i \pm}$ is the \pm 1 $\sigma$ changes in the rate uncertainty and $\mathrm{k}_{j i k}$ is for the shape uncertainty.

The Gaussian constraints for the background components with $4_{j}$ is,

$$
\mathrm{G}_{j}\left(\beta_{j} \mid 4_{, j}\right) / \frac{1}{\sqrt{2 \phi \cdot 4 \frac{2}{j}}} \exp \left(-\frac{1}{2} \cdot\left(\frac{\beta_{j}-1.0}{4}\right)^{2}\right)
$$

\subsection{Background Estimation}

The final estimate number of signal and background and observed data are shown in Table 6.1(already shown in chapter 4). After selection of events, the expected number of signal events is $15 \%$ of the backgrounds in $2 /$ et and $24 \%$ in 3 et.

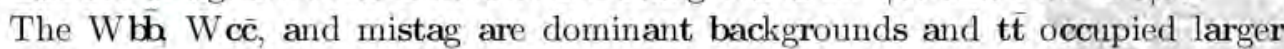
portion in $3 \mid$ et than $2 \mid$ et. These estimated numbers are used to the likelihood fit. 


\begin{tabular}{|c|c|c|}
\hline & 2|ets & $3 \mid$ ets \\
\hline s-channel & $72.7 \pm 10.5$ & $23.2 \pm 3.3$ \\
t-channel & $111.4 \pm 16.5$ & $32.4 \pm 4.8$ \\
Wbb & $906.9 \pm 273.4$ & $259.5 \pm 78.4$ \\
Wcc+Wc & $755.25 \pm 232.9$ & $192.1 \pm 59.2$ \\
Mistag & $681.6 \pm 95.4$ & $195.3 \pm 27.9$ \\
ttbar & $246.75=35.8$ & $559.65 \pm 81$ \\
Z+lets & $41.85 \pm 6.4$ & $16.2 \pm 2.4$ \\
Diboson & $117.45 \pm 12.15$ & $37.1 \pm 3.9$ \\
non-W & $123.3 \pm 49.35$ & $49.8 \pm 19.9$ \\
\hline Total Signal & $184.1 \pm 27.0$ & $55.6 \pm 8.1$ \\
\hline Total Prediction & $3057 \pm 519.3$ & $1365.3 \pm 162.4$ \\
\hline Observed in Data & 2920 & 1300 \\
\hline
\end{tabular}

Table 6.1. Data and Background for single-top production with $4.8 \mathrm{fb}^{-1}$

\subsection{Systematic Uncertainties}

Source of systematic uncertainty are incorporated in this analysis as nuisance parameters in the likelihood function, conform with a fully Bayesian treatment. Most systematic uncertainties, such as the uncertainty on the let-energy-scale, can affect the analysis in two ways. first, since we require the lets to have $\mathrm{E}_{T}$ $>15 \mathrm{GeV}$, a change in the let-energy-scale affects the event detection efficiency . Meanwhile, the shape of the $\mathrm{H}_{T}$ distribution, which depends also on let energies, will also change(we call this the 'shape uncertainty'). We include all sources of systematic uncertainty into the likelihood and take the correlation between normalization and shape uncertainties for a given source into account.

\subsubsection{Rate Systematic Uncertainties}

Normalization uncertainties are estimated by recalculating the event detection efficiency using Monte Carlo samples altered due to a specific systematic effect. The normalization uncertainty is the difference between the systematically shifted event detection efficiency and the default event detection efficiency. The effect of the uncertainty in the let energy scale is evaluated by applying let-energy corrections that describe $=\sigma$ variations to the default correction factor. For the s-channel, both variations show a lower event detection efficiency(both relative

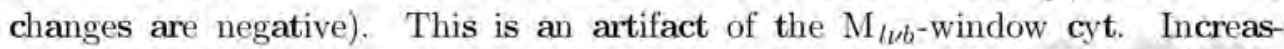
ing(decreasing) the let energy scale factor by the $+1 \sigma(-1 \sigma)$ variation, shifts the $\mathrm{M}_{l \mu b}$ distribution to higher(lower) values. Systematic uncertainties due to the 
modeling of ISR and FSR was increased and decreased in the parton showering to represent $\pm \sigma$ variations.

Jet Energy Scale(JES) The uncertainties in the let energy scale are estimated by processing all events in Monte Carlo samples with the let energy scale varied with $\pm 1 \sigma$. The correction of let energy is to dertermine the energy correction to scale the measured energy of the let energy back to the energy of final state particle level |et. Also, there are additional corrections to the measured |et energy to the parent parton energy. The let energy corrections are performed through the multi-levels; detector non-uniformity, multiple interactions, not assigned let outside of the let cone and so on. The original parton transverse energy can be estimated as follows [48].

$$
\mathrm{p}_{T}^{\text {parton }} /\left(\mathrm{p}_{T}^{\text {jet }} \times \mathrm{C}_{\eta}-\mathrm{C}_{M I}\right) \times \mathrm{C}_{A b s}-\mathrm{C}_{U E}+\mathrm{C}_{O O C} / \mathrm{p}_{T}^{\text {particle }}-\mathrm{C}_{U E}+\mathrm{C}_{O O C}
$$

where $p_{T}^{\text {parton }}$ is the transverse momentum of the parent parton, $\mathrm{p}_{T}^{j e t}$ is the transverse momentum measured in the calorimeter $\mid$ et and $p_{T}^{\text {particle }}$ is the particle let momentum.

- $\mathrm{C}_{\eta}$ - " $\eta$-dependent" correction for homogeneous response.

- $\mathrm{C}_{M I}$. "Multiple Interaction" correction for sibtracting the let from multiple $\mathrm{pp}$ interactions.

- $\mathrm{C}_{A b s}$. "Absolute" correction of the calorimeter response to the momentum of the particle let.

- $\mathrm{C}_{U E}$ and $\mathrm{C}_{O O C}$. "Underlying Event" and "Out-Of-Cone" corrections for parton radiation and hadronization effects due to the finite size of the let cone algorithm.

All corrections are determined as the transverse momentum of let but they apply to all components of the four-momentum of the |et. The uncertainty of |et energy scale is about $3 \%\left(\mathrm{P}_{T}>70 \mathrm{GeV}\right)$ and $3 \sim 9\left(\mathrm{P}_{T}<70 \mathrm{GeV}\right)$ in Figure 6.1. The systematic error from JES about single-top cross section is about $+8 /-11 \%$.

Heavy Flavor Fraction in $\mathrm{W}+$ ets The uncertainty about the prediction of Wbb Woc, Wc mainly comes from Heavy flavor fraction(k-factor).

The heavy flavor(HF) content of the $\mathrm{W}+$ ets sample is calculated by [35] [36].

$$
\mathrm{N}_{W+H F}^{\text {tag }} / \mathrm{N}_{W+\text { jets,data }}^{\text {pretag }} \times \mathrm{F}_{H F} \times \epsilon_{t a g}^{W+H F} \times \mathrm{KF}
$$

where $\mathrm{F}_{H F} / \mathrm{N}_{b, M C}^{W+\text { Jets }} / \mathrm{N}_{\text {jets, MC }}^{W+\text { Jets }}$ is the heavy flavor fraction of the $\mathrm{W}+$ ets sample, $\epsilon_{\operatorname{tag}}^{W+H F}$ is the tagging efficiency in the W+HF Monte Carlo sample, $\mathrm{N}_{W+j \text { cts,data }}^{\text {pretag }}$ is the number of pretag $\mathrm{W}+$ ets events in data, and KF is the calibration factor(or K-factor) of the heavy flavor fraction $\left(\mathrm{F}_{H F}\right)$. The K-factor is 


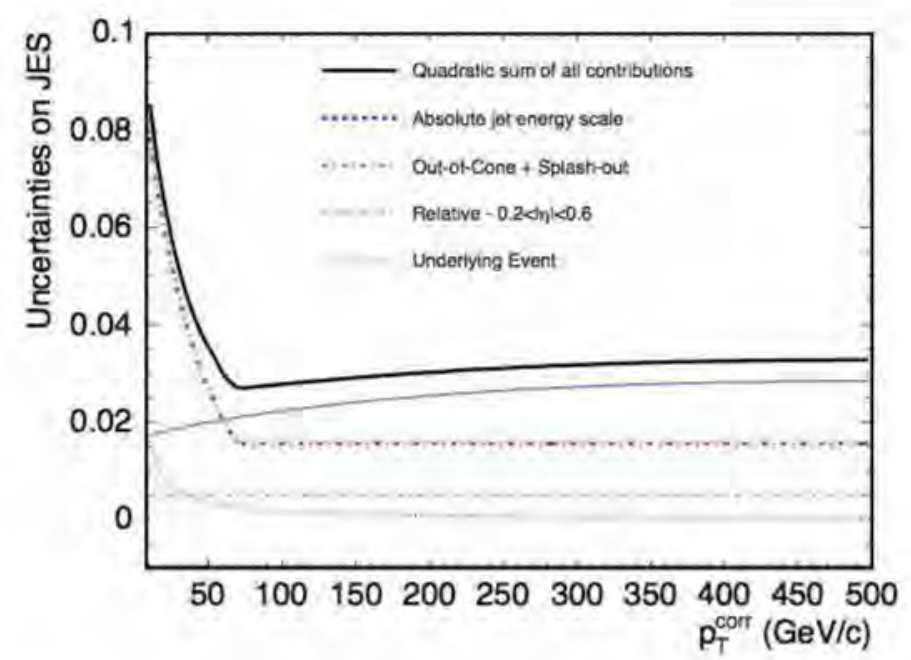

Figure 6.1. The Uncertainty as a function of the corrected $\mid$ et $\mathrm{P}_{T}$ in $0.2<\eta<0.6$

defined as

$$
\mathrm{KF} / \frac{\mathrm{F}_{j, \text { data }}^{b, \text { events }}}{\mathrm{F}_{j, \text { MC }}^{b, \text { events }}}
$$

where $\mathrm{F}_{j, \text { data }}^{\text {b,events }} / \mathrm{N}_{b, \text { data }} / \mathrm{N}_{\text {jets,data }}$.

The prediction of the $\mathrm{W} \mathbf{b} \mathbf{b}, \mathrm{Wc}$, and $\mathrm{Wc}$ fractions in the $\mathrm{W}+2$ |ets and $\mathrm{W}+3$ |ets samples are extrapolation from the $\mathrm{W}+1$ |et sample. The ALPGEN underpredicts the $\mathbf{W} \mathbf{b} \mathbf{b} \mathbf{W} \mathbf{c} \bar{c}$ fractions in the $\mathrm{W}+1$ et sample by a factor of $1.4 \pm$ 0.4 [37] (Ideally, it should be unity); this effect comes from the incorrect model of gluon splitting in the MC and Higher order effects. The template fits to the $\mathrm{W}+1$ |et bin data using KNN flavor separator are shown in Figure 6.2. The Wc prediction from ALPGEN is compared to measurement [38] and does not require scaling, but uncorrelated uncertainty is assigned to the Wc prediction. The Uncertainties from $\mathrm{KF}$ is about $30 \%$ and the systematic error about single-top cross section is $+12 /-12 \%$ for $\mathrm{Wbb}+\mathrm{Wcc}$ and $+1 /-9 \%$ for $\mathrm{Wc}$.

Luminosity The uncertainty $( \pm 6 \%)$ of integrated luminosity comes from the inelastic pp cross section and the acceptance of luminosity monitor.

Luminosity is measured by the Cherenkov Luminosity Counters(CLC) [49] [60]. The CLC measure the average number of interactions per bunch crossing $(\mu)$ and 


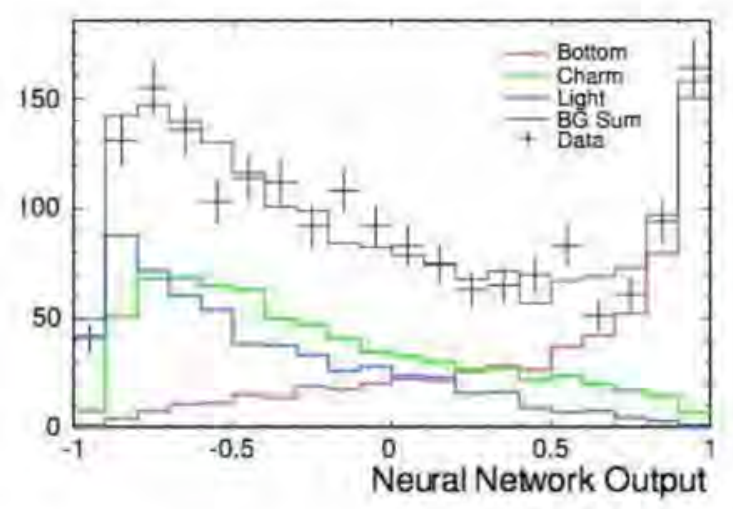

Figure 6.2. Template fits to the $\mathrm{W}+1$ |et bin data to extract the fraction of tagged lets using KNN flavor separator

the instantaneous luminosity is calculated by.

$$
\text { L } \frac{\mu \cdot \mathrm{f}_{B C}}{\sigma_{t o l}}
$$

where the $\sigma_{t o t}$ is the total p p cross-section, $\mathrm{f}_{B C}$ is the rate of bunch crossings. The uncertainty by luminosity monitor comes from following effects; Geometry, Generator, Beam Position, CLC simulation. SPP(single particle peak) calibration, Acceptance stability, Online $\rightarrow$ Offline transfer, Backgrounds (Table 6.2). Uncertainty of luminosity is $6 \%$ and systematic error is $+12 /-12 \%$.

Theoretical Cross Sections The signal and background Monte Carlos underpredict the total cross sections, so the predictions need to be scaled to the NLO theoretical models. The effects of the top quark mass is separated out from the sources of uncertainty affecting the theoretical predictions. Figure 6.3 shows the cross-section of $t \bar{t}$ and its uncertainties versus top mass. The uncertainty of theoretical $t \bar{t}$ cross-section at top mass $175 \mathrm{GeV}$ is about $12 \%$. The systematic error about single-top cross section is $+1 /-9 \%$.

Initial/Final State Radiation The ISR [40] and FSR [40] systematic uncertainties are estimated by altering samples which are more or less initial-state radiation(ISR) and final-state radiation(FSR). The effects of variations of ISR and FSR are taken to be correlated. Figure 6.4 shows $\mathrm{P}_{t}$ distribution of the dileptons for ISRmore and ISRless [39]. The Uncertinty of ISR and FSR is $15 \%$ and systematic error is $+5 /-10 \%$. 


\begin{tabular}{|c|c|}
\hline Sytematic Effect & Updated Uncertainty \\
\hline Geometry & $3 \%$ \\
Generator & $2 \%$ \\
Beam Position & $<1 \%$ \\
CLC simulation & $1 \%$ \\
SPP calibration & $<1 \%$ \\
\hline Acceptance stability & $1 \%$ \\
Online $\rightarrow$ Offline transfer & negligible \\
Backgrounds & $<1 \%$ \\
Statistical uncertainty & negligible \\
Luminosity method & negligible \\
\hline \hline Total by CLC measurement & $<4.2 \%$ \\
\hline \hline Inelastic pp cross section & $4 \%$ \\
\hline
\end{tabular}

Table 6.2. Uncertainty of Luminosity from Various Sources

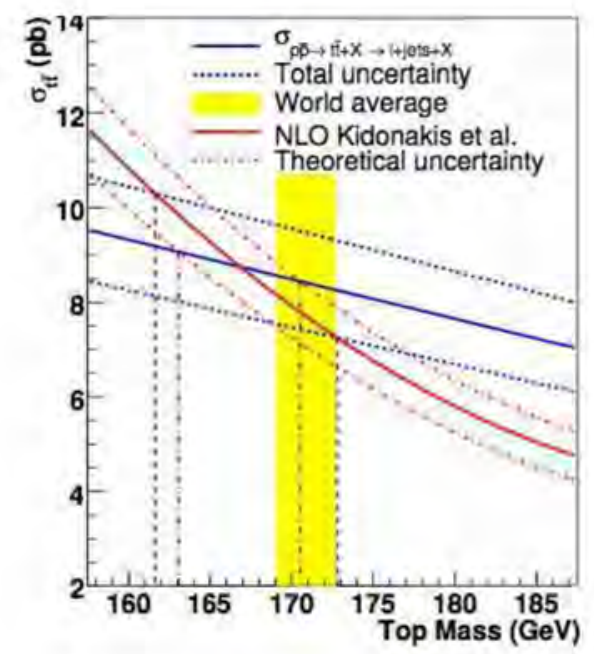

Figure 6.3. The cross-section and its uncertainty of $\mathbf{t} \overline{\mathbf{t}}$ cross section versus top mass 


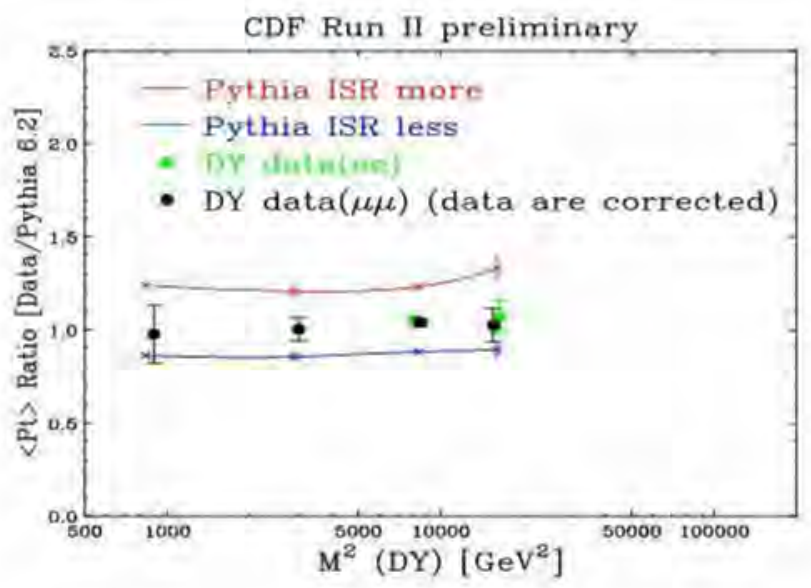

Figure 6.4. The Uncertianty of Initial State Radiation

Accecptance and Efficiency Scale Factors The mismodeling of the lepton identification [33] and the b-tagging efficiency [34] affects the predicted rates of signal and backgrounds. The uncertainties are considered by the scale factors for correction between the simulation and the data.

Parton Distribution Function The PDF parameterization systematic was studied by re-weighted single top and $t \bar{t}$ events with weights associated with default PDF CTEQ5L [32], and PDFs from MRST72, MRST75 and CTEQ6M. CTEQ6M is 20 orthogonal CTEQ PDF sets with up and down for $90 \%$, MRST72 is MRST PDF set with same $\alpha_{s}$ value as CTEQ5L and MRST75 is MRST PDF set with different $\alpha_{s}$ value. The uncertainties is calculated by the quadrature of differences between PDF sets. Some compared PDF is shown in Figure 6.5.

The Table 6.3 shows the summary of Systematic rate Uncertainties and Error. The Table 6.46 .5 shows the systematic rate uncertainties for 2 et and $3 \mid$ et bin, in percent. 


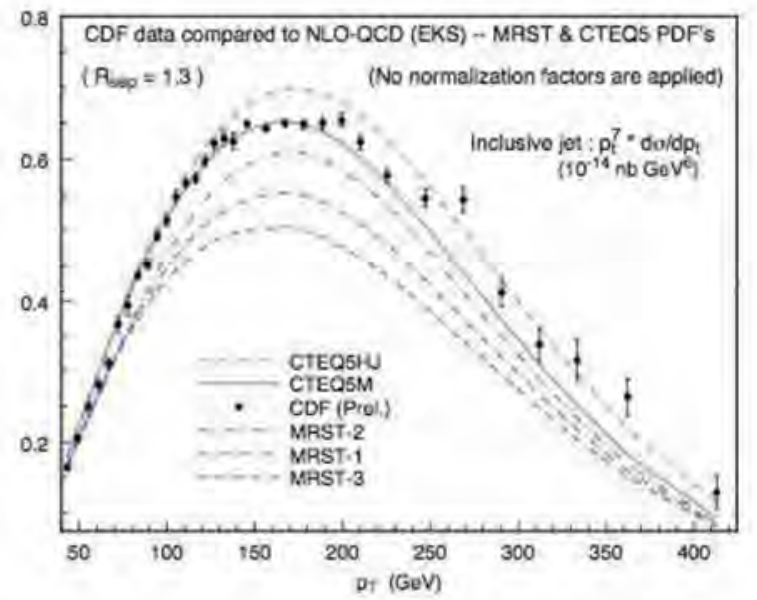

Figure 6.5. The Comparison of the Parton Distribution Functions

\begin{tabular}{|c|c|c|}
\hline Source & Uncertainty & Error \\
\hline \hline Jet Energy Scale & $\begin{array}{c}3 \%(>70 \mathrm{GeV}) \\
3 \sim 9 \%(<70 \mathrm{GeV})\end{array}$ & $+8 /-11 \%$ \\
\hline Initial + Final State Radiation & $15 \%$ & $+5 /-10 \%$ \\
\hline signal+tt nomalization due to PDF & $2 \sim 3 \%$ & $+5 /-6 \%$ \\
\hline ttbar cross section & $12 \%$ & $+1 /-9 \%$ \\
\hline Monte Carlo Generator & $5 \%$ & $+1 /-9 \%$ \\
\hline Event Detection Efficiency & $5 \sim 8 \%$ & $+7 /-11 \%$ \\
\hline Luminosity & $6 \%$ & $+12 /-12 \%$ \\
\hline Wbb+Wcc normalization (k/ factor) & $30 \%$ & $+12 /-12 \%$ \\
\hline Wc normalization & $30 \%$ & $+1 /-9 \%$ \\
\hline Total & & $+13 /-16.2 \%$ \\
\hline
\end{tabular}

Table 6.3. Systematic rate Uncertainties and Error 


\begin{tabular}{|c|c|c|c|c|c|}
\hline Source & t-channel & s-channel & $\mathrm{tt}$ & $\mathrm{W}+\mathrm{LF}$ & \\
\hline JES - $/+$ & $-1.1 / 0.6 \mathrm{~s}$ & $-0.1 /-0.6 \mathrm{~s}$ & $9.7 /-9.3 \mathbf{s}$ & $\mathrm{s}$ & \\
\hline ISR more/less & $-0.2 / 2.8 \mathrm{~s}$ & $6.7 / 0.3 \mathrm{~s}$ & $-7.1 /-2.6 \mathrm{~s}$ & & \\
\hline FSR more/less & $-1.3 / 4.2 \mathrm{~s}$ & $0.4 / 5.9 \mathrm{~s}$ & $-2.6 /-5.1 \mathrm{~s}$ & & \\
\hline PDF & $-3.5 / 3.1 \mathrm{~s}$ & $-1.4 / 1.7 \mathrm{~s}$ & $-2.3 / 1.9 \mathrm{~s}$ & & \\
\hline Signal MC model & $-2.0 / 2.0$ & $-1.0 / 1.0$ & & & \\
\hline Luminosity & $-6.0 / 6.0$ & $-6.0 / 6.0$ & $-6.0 / 6.0$ & & \\
\hline Efficiency & $-4.2 / 4.2$ & $-2.3 / 2.3$ & $-2.6 / 2.6$ & & \\
\hline Cross section ( $t$ ) & $-12.6 / 12.6$ & $-12.4 / 12.4$ & & & \\
\hline Cross section (tt) & & & $-12.4 / 12.4$ & & \\
\hline $\mathrm{m}_{t}(170 / 180)$ & $6.1 /-5.3 \mathrm{~s}$ & $9.5 /-8.0 \mathrm{~s}$ & $7.8 /-8.1 \mathrm{~s}$ & & \\
\hline$\eta_{j 2}$ & $\mathrm{~s}$ & $\mathrm{~s}$ & $\mathbf{s}$ & $\mathrm{s}$ & \\
\hline $4 \mathrm{R}_{j, j}$ & $\mathbf{s}$ & $\mathrm{s}$ & $\mathrm{s}$ & $\mathrm{s}$ & \\
\hline DataMistags & & & & $\mathrm{s}$ & \\
\hline KIT Flav, Sep. & & & & $\mathrm{s}$ & \\
\hline & $\overline{\mathrm{Wbb}}$ & $\overline{W c}(\mathbf{c})$ & Diboson & $Z+$ ets & Non-W \\
\hline JES - $/+$ & $6.9 /-7.6 \mathrm{~s}$ & $7.0 /-6.3 \mathrm{~s}$ & $-2.6 / 1.7 \mathrm{~s}$ & $-5.3 / 5.4 \mathrm{~s}$ & \\
\hline Luminosity & & & $-6.0 / 6.0$ & $-6.0 / 6.0$ & \\
\hline Efficiency & & & $-9.0 / 9.0$ & $-8.3 / 8.3$ & \\
\hline Cross section (dib) & & & $-2.0 / 2.0$ & & \\
\hline$Z+\mid$ ets rate & & & & $-10.8 / 10.8$ & \\
\hline $\mathrm{W}+\mathrm{QQ}$ k-factor & $-30.1 / 30.1$ & $-13.9 / 13.9$ & & & \\
\hline W+c k-factor & & $-16.1 / 16.1$ & & & \\
\hline$\eta_{j 2}$ & $\mathbf{s}$ & $\mathrm{s}$ & $\mathrm{s}$ & $\mathrm{s}$ & \\
\hline Non-W rate & & & & & $-40.0 / 40.0$ \\
\hline Non-W Flavor & & & & & $\mathrm{s}$ \\
\hline
\end{tabular}

Table 6.4. Systematic rate uncertainties for 2-let bin. Shape uncertinties are indicated with an "s" 


\begin{tabular}{|c|c|c|c|c|c|}
\hline Source & t-channel & s-chamnel & $\mathrm{tt}$ & $\mathrm{W}+\mathrm{LF}$ & \\
\hline JES - $/+$ & $-9.7 / 2.5 \mathrm{~s}$ & $-8.0 / 8,9 \mathrm{~s}$ & $4.6 /-5.1 \mathrm{~s}$ & s & \\
\hline ISR more/less & $-0.5 /-4.5 \mathrm{~s}$ & $-12.6 / 2.9 \mathrm{~s}$ & $-5.0 /-0.6 \mathrm{~s}$ & & \\
\hline FSR more/less & $-2.5 /-0.8 \mathrm{~s}$ & $-4.8 /-6.3 \mathrm{~s}$ & $-2.3 / 3.5 \mathrm{~s}$ & & \\
\hline PDF & $-3.7 / 3.3 \mathrm{~s}$ & $-1.5 / 1.7 \mathrm{~s}$ & $-2.3 / 1.9 \mathrm{~s}$ & & \\
\hline Signal MC model & $-1.5 / 1.5$ & $-1.9 / 1.9$ & & & \\
\hline Luminosity & $-6.0 / 6.0$ & $-6.0 / 6.0$ & $-6.0 / 6.0$ & & \\
\hline Efficiency & $-4.3 / 4.3$ & $-3.9 / 3.9$ & $-4.0 / 4.0$ & & \\
\hline Cross section (t) & $-12.6 / 12.6$ & $-12.4 / 12.4$ & & & \\
\hline Cross section (tt) & & & $-12.4 / 12.4$ & & \\
\hline $\mathrm{m}_{t}(170 / 180)$ & $6.4 /-6.0 \mathrm{~s}$ & $11.7 /-8.6 \mathrm{~s}$ & $9.3 /-8.6 \mathrm{~s}$ & & \\
\hline$\eta_{j 2}$ & $\mathbf{s}$ & $\mathbf{s}$ & $\mathrm{s}$ & $\mathrm{s}$ & \\
\hline $4 \mathrm{R}_{j, j}$ & $\mathrm{~s}$ & $\mathbf{s}$ & $\mathrm{s}$ & $\mathrm{s}$ & \\
\hline DataMistags & & & & $\mathrm{s}$ & \\
\hline KIT Flav. Sep. & & & & $\mathrm{s}$ & \\
\hline & $\overline{\text { Wbb }}$ & $\overline{W c}(\mathbf{c})$ & Diboson & $Z+\mid$ ets & Non-W \\
\hline JES - / + & $8.8 /-8.8 \mathrm{~s}$ & $8.3 /-7.1 \mathrm{~s}$ & $-13.0 / 10.8 \mathrm{~s}$ & $-10.5 / 13.6 \mathrm{~s}$ & \\
\hline Luminosity & & & $-6.0 / 6.0$ & $-6.0 / 6.0$ & \\
\hline Efficiency & & & $-9.0 / 9.0$ & $-8.0 / 8.0$ & \\
\hline Cross section (dib) & & & $-2.0 / 2.0$ & & \\
\hline$Z+\mid$ ets rate & & & & $-10.8 / 10.8$ & \\
\hline $\mathrm{W}+\mathrm{QQ}$ k-factor & $-30.1 / 30.1$ & $-17.7 / 17.7$ & & & \\
\hline $\mathrm{W}+\mathbf{c}$ k-factor & & $-12.3 / 12.3$ & & & \\
\hline$\eta_{j 2}$ & $\mathbf{s}$ & $\mathbf{s}$ & $\mathrm{s}$ & $\mathrm{s}$ & \\
\hline Non-W rate & & & & & $-40.0 / 40.0$ \\
\hline Non-W Flavor & & & & & $\mathrm{s}$ \\
\hline
\end{tabular}

Table 6.5. Systematic rate uncertainties for 3-|et bin. Shape uncertinties are indicated with an "s" 


\subsubsection{Shape Systematic Uncertainties}

Compared with rate uncertainties, shape systematic uncertainties are considered as shape distortions of the templates for the signals and backgrounds. All shape uncertainties are median smoothed with five bins except the first and last two bins. The bins of ratio historam, which is divided the sytematically varied histogram by the central histogram, are replaced by median of 5 bins(itself and neighboured bins). This prodedure play a role in removing extreme outliers and high frequency noise. The smoothed ratio histograms are then multiplied by the central histograms to obtain the new varied histograms. The shape systematic sources are JES, PDF, ISR/FSR, Jet Flavor Separator Modeling, Mistag Model, Non-W Flavor Composition, Jet $\eta$ Distribution, Jet $4 \mathrm{R}_{j j}$ Distribution.

Jet Flavor Separator M odeling For verifying tagged $\mathbf{b}$-let, the neural-network let flavor separator is used. The predicted shape of the flavor-separator for tagged b lets is well modeled by the simulation, but the light-flavor sample was found to need a small correction about the NN distribution. The difference between the uncorrected light-falvor Monte Carlo prediction and the data-derived corrected distribution is taken as the shape systematic uncertainty. Because the pure sample of charm lets can't be derived from the data, the shape template for $\mathrm{W}+$ charm lets is estimated from the difference between the distribution of Monte Carlo simulation and the alterd Monte Carlo distribution by the light-flavor correction.

Mistag Model The untagged data largely consist of W+light falvor lets, but $\mathrm{W} \overline{\mathbf{b}} \mathbf{W} \boldsymbol{W} \bar{c}, \mathrm{t} \bar{t}$, and single top events are also contained. The shape template for mistag model is made from the untagged data weighted by the mistag matrix weights.

Non-W Flavor Composition The neural-network flavor separator distribution is used to fit the flavor fractions in the low- $\mathrm{E}_{T}$ control samples. The uncertainty in expected flavor composition of the non-W events comes from the limited statistical precision of these fits and the necessity of extrapolating to the higher- $\mathbb{E}_{T}$ signal region. The central-value estimates are $40 \% \mathbf{b}$ |ets, $45 \% \mathbf{c}$ |ets, and $15 \%$ light-flavor lets. A systematic sample uses the variation of the flavor composition. $60 \%$ b ets, $30 \%$ c lets, and $10 \%$ light-flavor lets,

Jet $\eta$ Distribution The mismodeling of $\mid$ ets at high $|\eta|$ region is checked with the untagged $\mathrm{W}+2 \mid$ ets control sample. This mismodeling affects the analysis relating to one of important input variables, $\mathrm{Q} \times \eta$. All Monte Carlo samples are weighted by a factor of the pseudorapidity of the second-leading let in $\mathrm{W}+2$ let 
èvents.

Jet $4 \mathrm{R}_{j j}$ Distribution The uncertainty in the distribution of $4 \mathrm{R}$ of two |ets is similar to $\mid$ et $|\eta|$ distribution. This angular distribution is mismodeled in the untagged control sample and the gluon splitting fraction in alpgen may affect this mismodeling. All Monte Carlo samples are weighted by using the ratio pf the untagged data to the prediction.

Figure [6.6][6.7] shows JES shape systematic compared plot between central and systematic sample. ALL shape systematic plots are located in Appendix C.
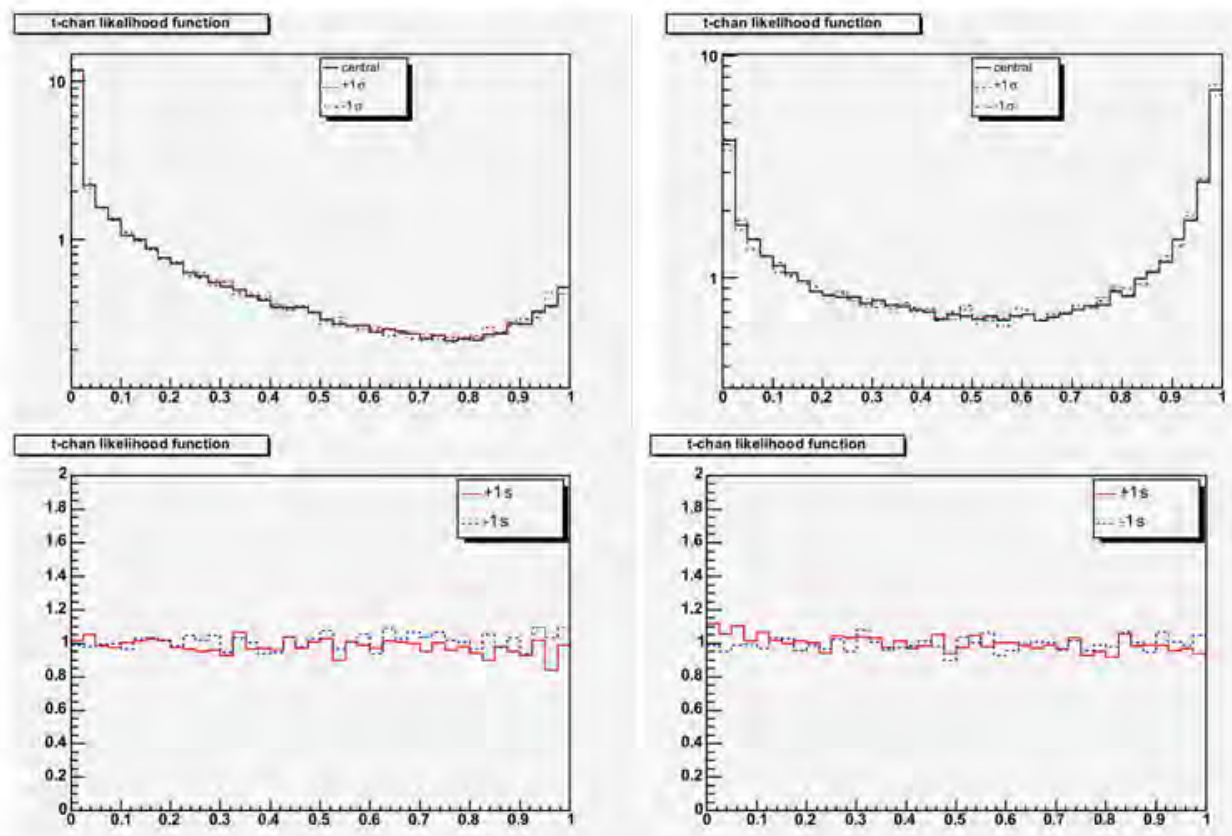

Figure 6.6. JES Shape Systematic Uncertainty(s-/t-channel); compared plot(up) and ratio plot(down) between central and systematic sample 

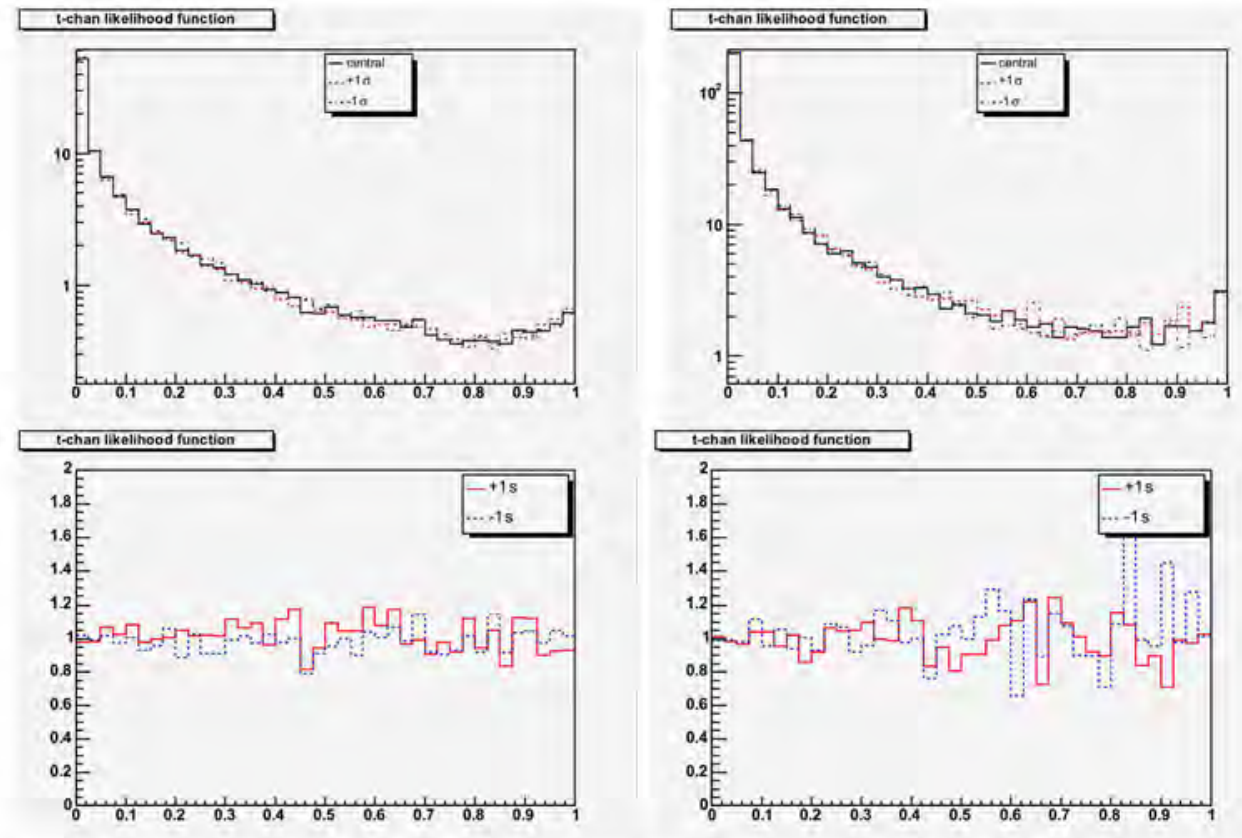

Figure 6.7. JES Shape Systematic Uncertainty(ttbar/Wbb); compared plot(up) and ratio plot(down) between central and systematic sample 


\subsection{Measurement of Cross Section}

The measured cross-section(Fig. [6.8]) is

$$
1.83+0.49(\text { stat })+0.49(\text { sys }) \quad(\mathrm{pb})
$$

by using $4.8 \mathrm{f} \mathrm{b}^{-1}$ of data collected by the CDF II experiment at the Fermilab Tevatron. we also set the limit(Fig.[6.9]) of

$$
V_{t b}>0.41 \text { at } 95 \% \mathrm{CL} \text {. }
$$

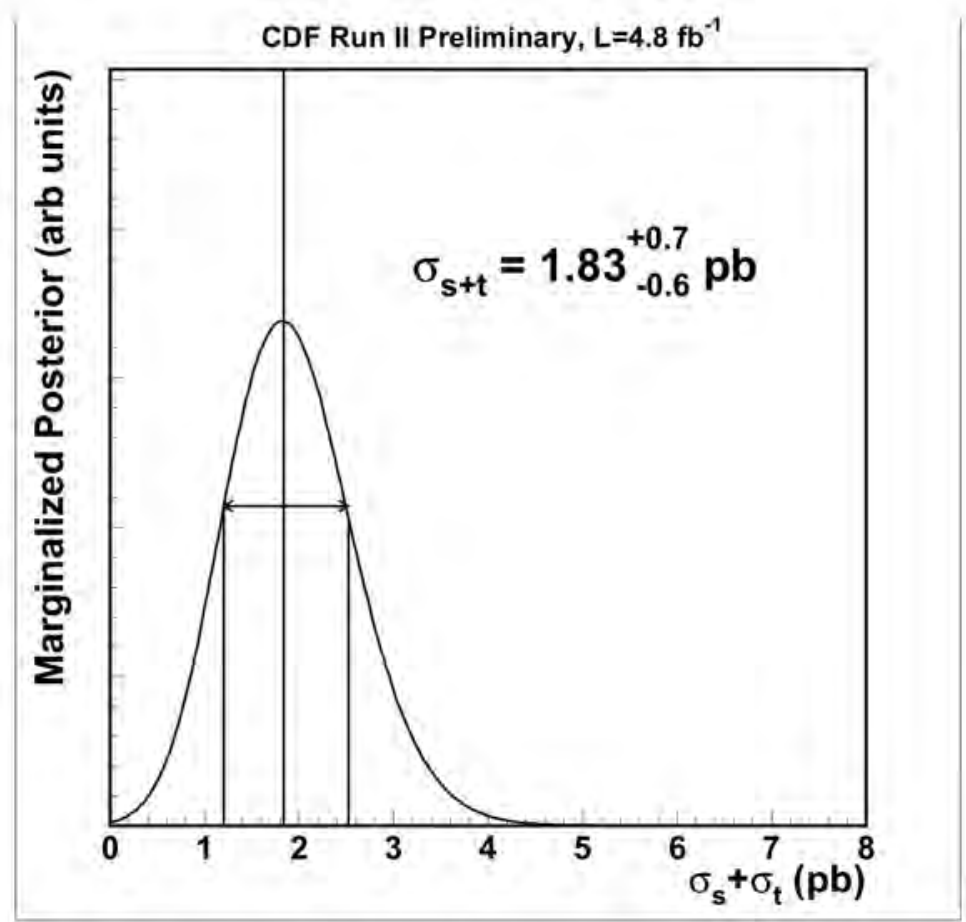

Figure 6.8. Fit for $\sigma_{s}+\sigma_{t}$. SM ratio of $\sigma_{s} / \sigma_{t}$ is assumed. The Baysian posterior, marginalized over nuisance parameter, is shown. The maximum value is the central value of the cross-section fit, and smallest interval enclosing $68 \%$ of the integral of the posterior is the quoted interval. The measured result is $1.83_{-0.43(\text { stat })-0.41(\text { sys })}^{+0.49(\mathrm{stat})+0.49(\text { sys })}(\mathrm{pb})$ 


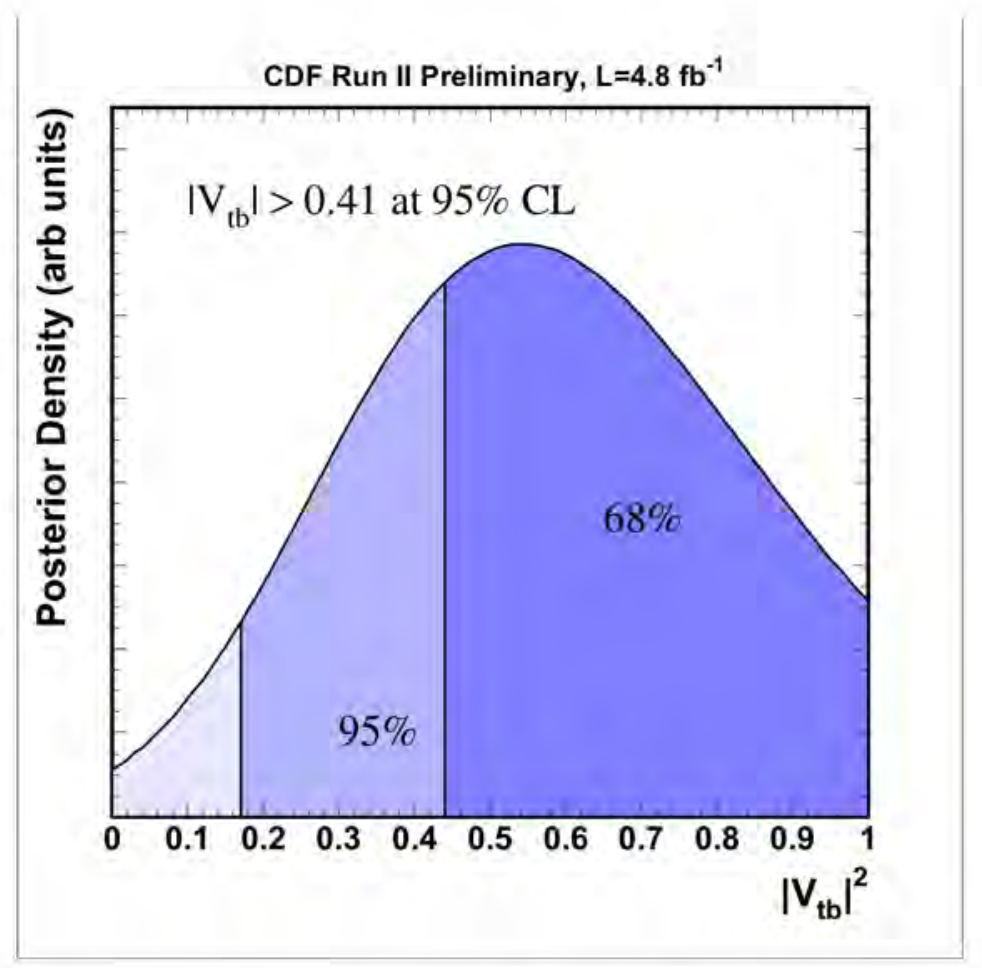

Figure 6.9. Extraction of limits on $\mid V \mathrm{tb}^{2}$ using a Baysian technique. The SM ratio of $\sigma_{s} / \sigma_{t}$ is assumed. The Baysian posterior, marginalized over nuisance parameter, including the theoretical uncertainty on the single-top cross section and uncertainties due to $\mathrm{m}_{t}$, is shown. The $68 \%$ and $95 \%$ confidence intervals are indicated with vertical lines. 


\subsection{Significance}

The p-value is the probability of obtaining a test statistic at least as extreme as the one actually observed against the null hypothesis. The data are compared with two hypotheses; test hypothese(signal+backgrounds) assumes single-top quark production and all backgorund processes while null hypothesis(background only) assumes Standard Model processes except single top quark production. We can test what hypothese the data prefer. For obtaining p-value, we calculate Q, the ratio of probability the data in the test $(\mathrm{s}+\mathbf{b})$ hypothesis to that in the null(b) hyothesis [48] [04] [45].

$$
\mathrm{Q} / \frac{\mathrm{P}(\text { data } \mid \mathbf{s}+\mathbf{b})}{\mathrm{P}(\mathrm{data} \mid \mathbf{b})} / \prod_{i=1}^{n_{\mathrm{ch}} n_{i}^{b i n s}} \prod_{j=1}\left[\frac{\mathrm{e}^{-\left(s_{i j}+b_{i, j}^{s}\right)}\left(\mathbf{s}_{i j}+\mathbf{b}_{i j}^{\mathrm{s}}\right)^{d_{i j}}}{\mathrm{~d}_{i j} \rightarrow}\right] /\left[\frac{\mathrm{e}^{-b_{i j}^{b}\left(\mathbf{b}_{i j}\right)^{d_{i j}}}}{\mathrm{~d}_{i j} \rightarrow}\right]
$$

The degree of evidence is quantified using pseudoexperiments. One set of pseudoexperiment is performed assuming test hypothese and the other assuming null hypothese. The pseudodata are made from Poisson random distribution with means of the sum of the backgrounds or signal+backgrounds, depending on the hypothesis.

Then the p-value is calculated as the probability of $-2 \ln Q<-2 \ln Q_{o b s}$, as suming the null hypothesis. The measured p-value correspoding the observed data is $9.07 \times 10^{-3}$.

The sensitivity of the analysis is computed as the median expected p-value assuming a signal is truly present. The median $-2 \ln Q$ is extracted from the test hypothesis, and the integral of the null distribution of $-2 \ln Q$ to the left of this median value is the median expected $\mathrm{p}$-value. The value thus obtained is $2.58 \times 10^{-5}$, correspoding to $4.05 \sigma$ using $3.2 \mathrm{f} \mathrm{b}^{-1}$. 


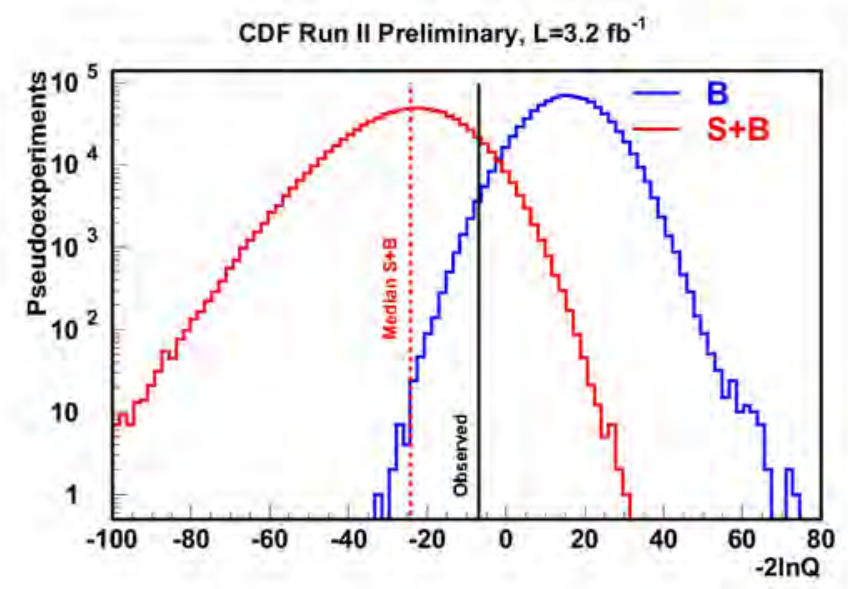

Figure 6.10. Plot of -2lnQ for the test hypothesis, in which pseudoexperiments are drawn from a model which assumes Standard Model backgrounds plus Standard Model single-top production, and for the null hypothesis, in which pseudoexperiments are drawn from a model which assumes single-top production is absent. The dashed line indicates median expected p-value of $2.58 * 10^{5}$ or $4.05 \sigma$ and solid line indicates the data, with a p-value of 0.009 or $2.36 \sigma$. 


\section{Chapter 7}

\section{Search for Anomalous Wtb Coupling}

\subsection{Anomalous Wtb Coupling}

In order to test the hypothesis that a fraction of single top quarks may be right-handed, we constructed a single-top events with MADEVENT [29]. The LO and NLO of t-channel samples need to be matched as fully differential NLO calculation[31].

To test anomalous Wtb coupling, we changed the handedness of the W tb coupling from the SM left-handed version $(V-\mathbf{A})$ to $V+\mathbf{A}$, for two of the four $\mathrm{W}$ boson vertices in each event. We chose to be the production $\mathrm{W}$ and not the decay $\mathrm{W}$. This is consistent with the available measurements of the $\mathrm{W}$ boson helicity in top quark decays [30], and borrows the singly-produced top quarks right-handed polarized. We call this the RRLL model (Figure 7.1).

Figure 7.2 shows the distributions of the cosine of the angle between the lepton and the down-type quark in the top quark rest frame. This angle is expected to show $\mathbf{a} 1+\cos \theta$ distribution for $100 \%$ polarized top quarks along this axis [11][1-2]. For s-channel production, the down-type quark is contained in one of the beams, while for t-channel production, $2 / 3$ of the time the down-type quark gives rise to a high- $\eta$ |et. Because we reconstruct the high- $\eta$ |et, and because t-channel production amounts to a higher fraction of single top quark production, we choose to use the angle $\cos \theta_{\ell j}$ in the reconstructed top quark frame,

The distribution of $\cos \theta_{\ell j}$ after reconstruction is shown in Figure 7.2. The right-hand side is sculpted by the lepton isolation cut. If the lepton is close to a reconstructed let, then it removed by the isolation requirement. For the RRLL sample, the leptons are more often found at large angels relative to the lets, and the isolation cut gives less effect. On the other hand, the RRLL samples have 

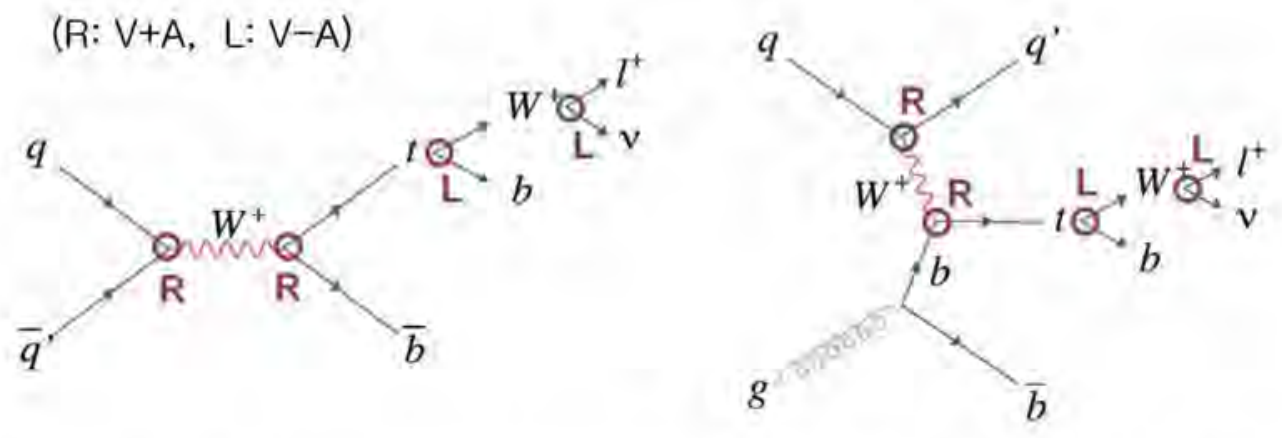

Figure 7.1. The RRLL sample's vertex coupling choices, "R" means $V+\mathbf{A}$ Wtb coupling, while "L" means $V$-A Wtb coupling.

leptons with a somewhat wider $\eta$ distribution than the SM case, and hence the distribution of $\cos \theta_{\ell j}$ is sculpted on the other side as well.

To test Wtb coupling using top polarization, the variable of $\cos \theta_{\ell j}$ is used to fit LLLL and RRLL sample. After applying cut of likelihood function $\left(\mathrm{L}_{t}>0.9\right)$, signal events become larger compared to background. This histogram is LLLL case, and RRLL will show the mirror image.

In order to measure separately the RRLL and SM signals, we divide the sample into two subsets. One subset is that events are treated in the region of $\cos \theta_{\ell j}>0$ and the others are $\cos \theta_{\ell j}<0$. Also two discriminants are used to measure the two cross sections. The $\cos \theta_{\ell j}>0$ sample is used to measure the SM (LLLL) cross section, and the $\cos \theta_{\ell j}<0$ sample is used to train a discriminant to measure the RRLL cross section. The basic fitting method is same as the privious likelihood fit. To extract $\sigma_{S M}$ and $\sigma_{R R L L}$, oint fit is carried out and the cross sections of each sample are measured. The correlation between the measured values of $\sigma_{S M}$ and $\sigma_{R R L L}$ are expected.

\subsection{Search Result}

A two-dimensional fit for the SM LLLL and exotic RRLL cross section is performed. The same technique is used as in the SM loint fits for the s- and t-channel production cross sections. The program xsfit2d, C is used, which calls the routine bh_2d_scan in mclimit_csm. C [28]. The same formalism is used as in the SM single top production cross section measurement. a Bayesian posterior is formed using a flat prior in the signal production cross section, and uncertain nuisance parameters are integrated over. In the two-dimensional case, a two-dimensional flat prior is chosen in the $\left(\sigma_{L L L L} ; \sigma_{R R L L}\right)$ plane. 

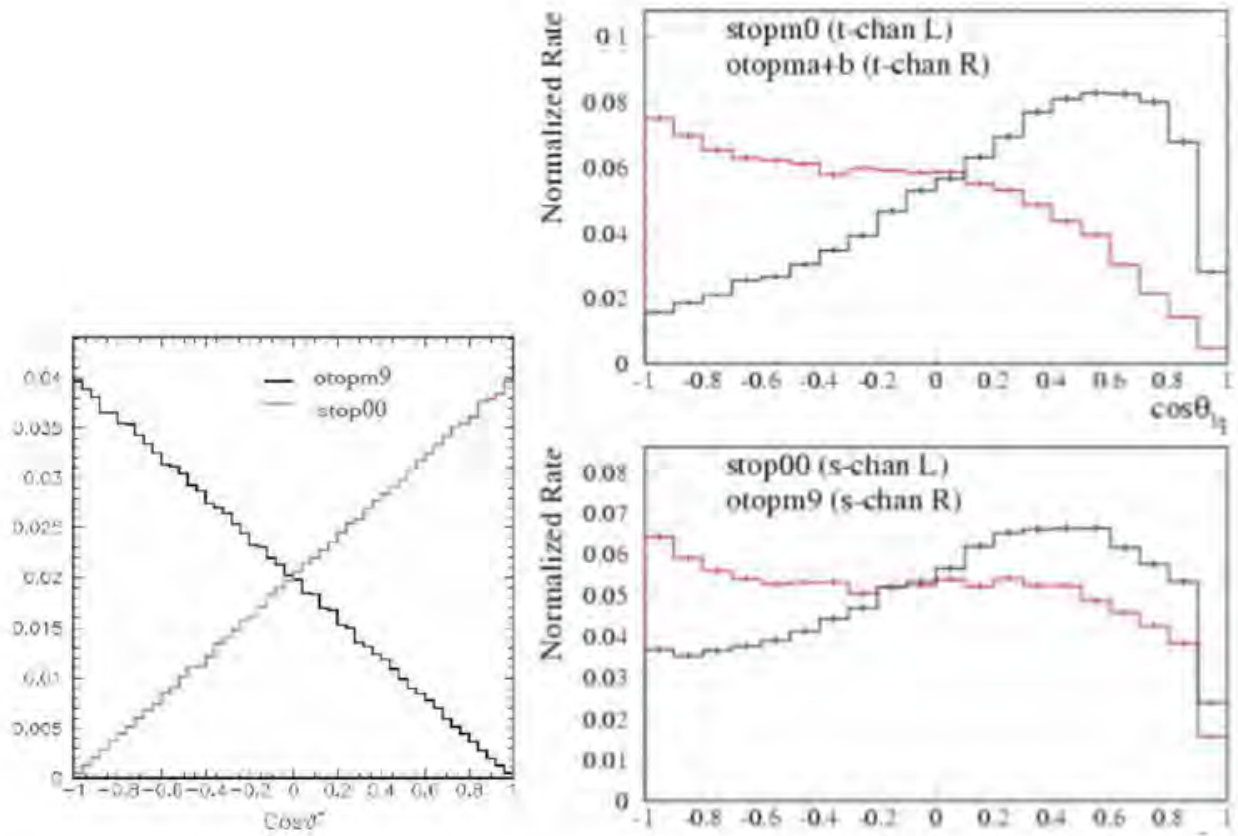

Figure 7.2. Left plot shows the distribution of $\cos \theta_{\ell q_{\text {beam }}}$ for the SM (stop00) and RRLL (otopm9) of s-channel. The $\cos \theta$ distributions of the SM and RRLL signal samples at the HEPG level has the opposite direction.

Right upper plot is the distributions of $\cos \theta_{\ell j}$ for t-channel signals of the SM (stopm0) and RRLL (otopma+b) after simulation, reconstruction, and event selection. The right bottom plot shows that the distribution of the s-channel at reconstruction level. 


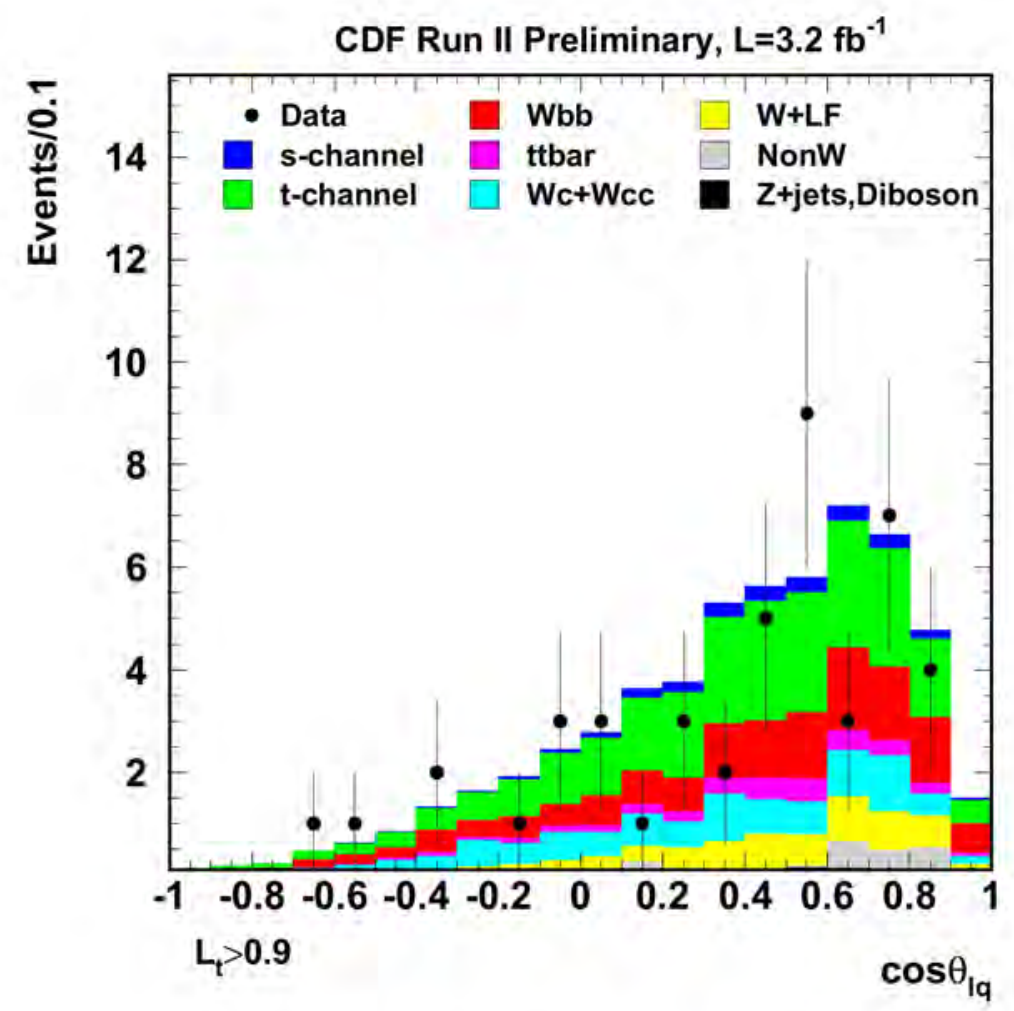

Figure 7.3. $\cos \theta_{l q}$ with $\mathrm{L}_{t}>0.9$ (signal. SM) 
In the general case, four production cross sections would have to be measured. The s-channel production may have a different polarization fraction from that in the t-channel production. We leave as a future exercise a more thorough exploration of the polarization-cross-section space. For now, we assume that the s-and t-channel production mechanisms have a total rate that is in the SM ratio. We do not constrain the total production rate to the SM rate, however.

We show the results of the 2D fit in Figure 7.4. $\beta$ means ratio of the measured and expected cross section. The best-fit values are $\sigma_{L} / 1.43 \mathrm{pb}$ and $\sigma_{R} / 0.0$ $\mathrm{pb}$. The green-shaded area indicates the region enclosing $68 \%$ of the integral of the posterior, and the yellow-shaded area indicates the region enclosing $95 \%$ of the integral of the posterior.

For the test of the anomalous $V$ tb coupling, the measured fraction of $V+\mathbf{A}$ coupling is

$$
V+\mathbf{A} \quad . \quad 0 \pm 28 \quad(\%) .
$$

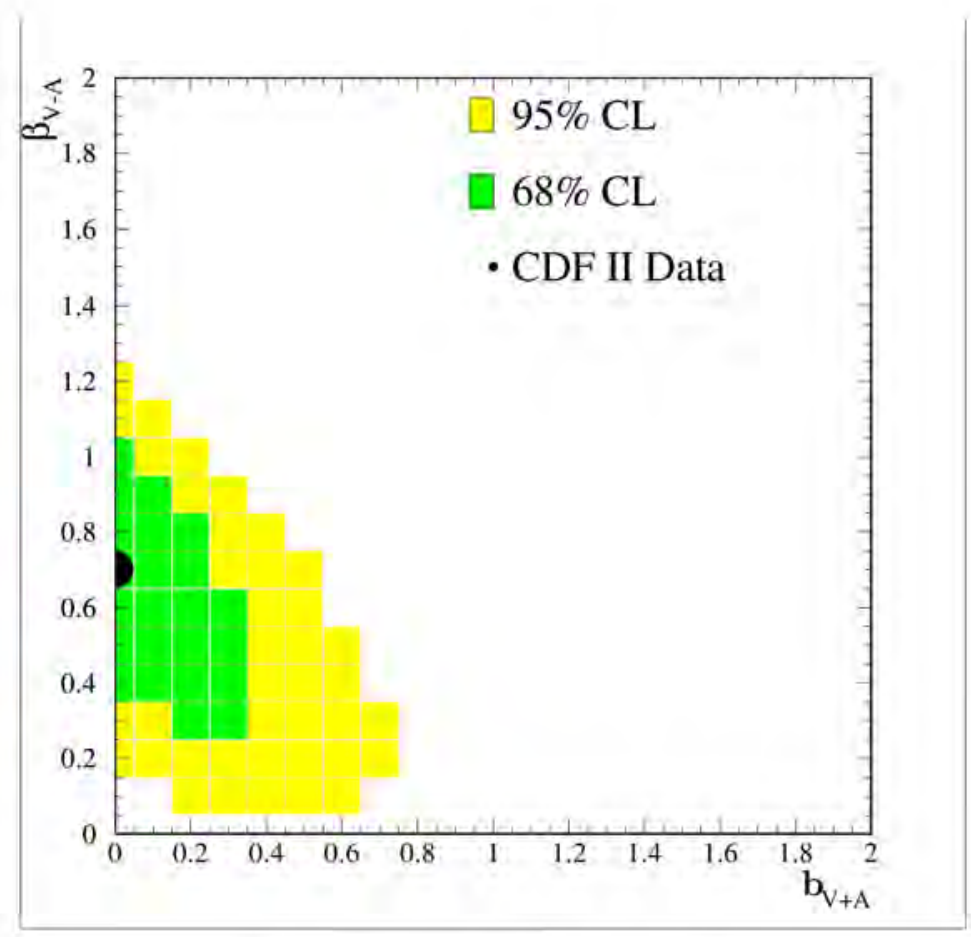

Figure 7.4. 2D Cross-section Fit 


\section{Chapter 8}

\section{Summary of Results and Discussion}

The top quark pair is produced via strong interaction and was discoverd in 1995 at the Tevatron. The standard model of elementary particle physics also predicts electroweak single top-quark production; s-channel, t-channel, associated tW production. The s-channel and t-channel process are dominant at Tevatron, so these two channels are considerd in the analysis.

Measuring the cross section of single-top production provides a direct determination of CKM matrix of $V_{t b}$. It is difficult to measure the single-top cross section because small expected cross-section and dominant background. To distinguish signal from background, likelihood method is used by combining several input variables. The likelihood ouput distribution of expected background process and the simulated SM single top-quark signal is compared to the distribution of observed events using Bayesian maximum likelihood technique.

This thesis describes the result of a measurement of single-top cross-section and a test of anomalous W-t-b coupling using $4.8 \mathrm{f} \mathrm{b}^{-1}$ of data collected by the CDF II experiment at the Fermilab Tevatron.

The measured cross-section is

$$
1.83 \underset{-0.43(\mathrm{stat})-0.41(\mathrm{sys})}{+0.49(\mathrm{stat})+0.49(\mathrm{sys})}(\mathrm{pb})
$$

we set the limit of

$$
V_{\mathrm{tb}}>0.41 \text { at } 95 \% \mathrm{CL} \text {. }
$$

For the test of the anomalous $V$ tb coupling, the measured fraction of $V+\mathbf{A}$ coupling is

$$
V+\mathbf{A} \quad \cdot 0=28 \quad(\%)
$$


The future of precistion top-quark phycics will take place at LHC. Expected single-top cross-section is $6.6 \mathrm{pb}( \pm 10 \%)$ for s-channel, $156 \mathrm{~b}(=10 \%)$ for t-channel and $34 \mathrm{pb}( \pm 10 \%)$ for associated production at $14 \mathrm{TeV}$. 


\section{Appendix A}

\section{Monitoring and Finding of Beam Position}

\section{A.1 Fitting Algorithm}

Primary Vertex The primary vertex is reconstructed using tracks with the vxprim module [27]. One module produces a vertex collection from svx and the other module produces a vertex from cot. Figure[A.1],[A.2] show beam position and slope in $x z$-plane and yz-plane fitted by using primary vertexes.

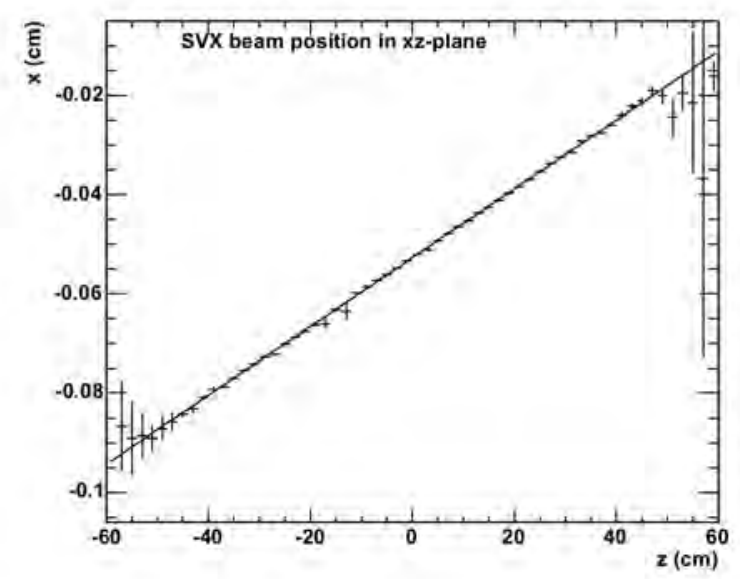

Figure A.1. Fitted SVX beam position in xz-plane using primary vertexes 


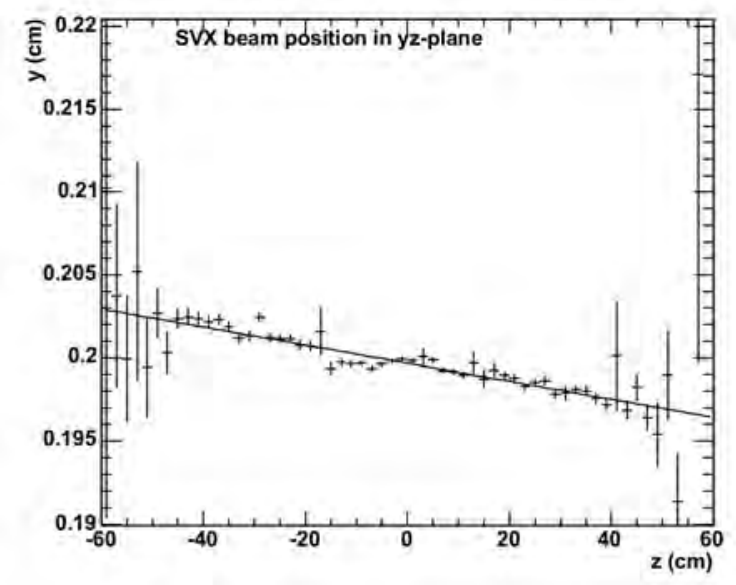

Figure A.2. Fitted SVX beam position in yz-plane using primary vertexes 
D- $\phi$ Correlation The impact parameter D of a track from a primary vertex at $\left(\mathrm{x}_{V} ; \mathrm{y}_{V}\right)$ can be parameterized.

$$
\mathrm{D}\left(\mathrm{x}_{v} ; \mathrm{y}_{v} ; \phi_{0}\right) /-\mathrm{x}_{v} \sin \phi_{0}+-\mathrm{y}_{v} \cos _{0}
$$

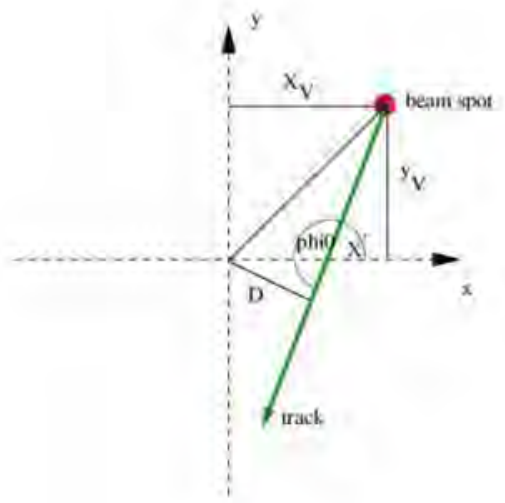

Using the $z_{0}$ parameter of the track and the beam line parameters $\vec{x}$ / $\left(\mathrm{x}_{0} ; \mathrm{y}_{0} ; \mathrm{x}_{\text {slope }} ; \mathrm{y}_{\text {slope }}\right) \mathrm{x}_{v}$ and $\mathrm{y}_{v}$ can be written as.

$$
\mathrm{x}_{v} / \mathrm{x}_{0}+\mathrm{z}_{0} \mathrm{x}_{\text {slope }} \mathrm{y}_{v} / \mathrm{y}_{0}+\mathrm{z}_{0} \mathrm{y}_{\text {slope }}
$$

The impact parameter D can be expressed,

$$
\mathrm{D}\left(\mathrm{z}_{0} ; \phi_{0}\right) / \overrightarrow{\mathrm{x}} \cdot \overrightarrow{\mathrm{g}}
$$

$\chi^{2} / \sum_{i=1}^{n}\left(\frac{\mathrm{D}_{i}-\overrightarrow{\mathrm{x}} \cdot \overrightarrow{\mathrm{g}}}{\sigma_{i}}\right)$, with $\sigma_{i}^{2} / \sigma_{\mathrm{D}_{i}}^{2}+2 \sigma_{\text {Beam }}^{2}$, with $\sigma_{i}^{2} / \sigma_{\mathrm{D}_{i}^{2}}+2 \sigma_{\text {Beam }}^{2}$ Minimizing this $\chi^{2}$, beam position can be obtained as follows.

$$
\overrightarrow{\mathrm{x}} / V \overrightarrow{\mathrm{b}}
$$

with

$$
V^{-1} / \sum_{i=1}^{N} \frac{\overrightarrow{\mathrm{g}}_{i} \overrightarrow{\mathrm{g}}_{i}^{T}}{\sigma_{i}^{2}} \text { and } \overrightarrow{\mathrm{b}} / \sum_{i=1}^{N} \frac{\mathrm{D}_{i} \overrightarrow{\mathrm{g}_{i}}}{\sigma_{i}^{2}}
$$




\section{A.2 Plots of Beam Position}

We studied beamlines for the silicon and cot about run range. Figure A.4 shows fit code for SVX and COT. Two constant values are stored; lower number means low statistical run. Figure A.5 and A.6 show the fitted value of beamline with $\mathrm{x}$-axis and $\mathrm{y}$-axis for $\mathrm{S} V \mathrm{X}$ and COT. Figure $\mathrm{A} 7$ is the value of difference of $\mathrm{SVX}$ and COT. We can check the tracking alignment from this plot. Annually, Silicon detector is sinking compared to COT little by little. Figure A.8 and A.9 are pull distributions of beam position of SVX-COT.

Run range is $222427 \sim 223642$ (period. 9/1/2007 9/26/2007).

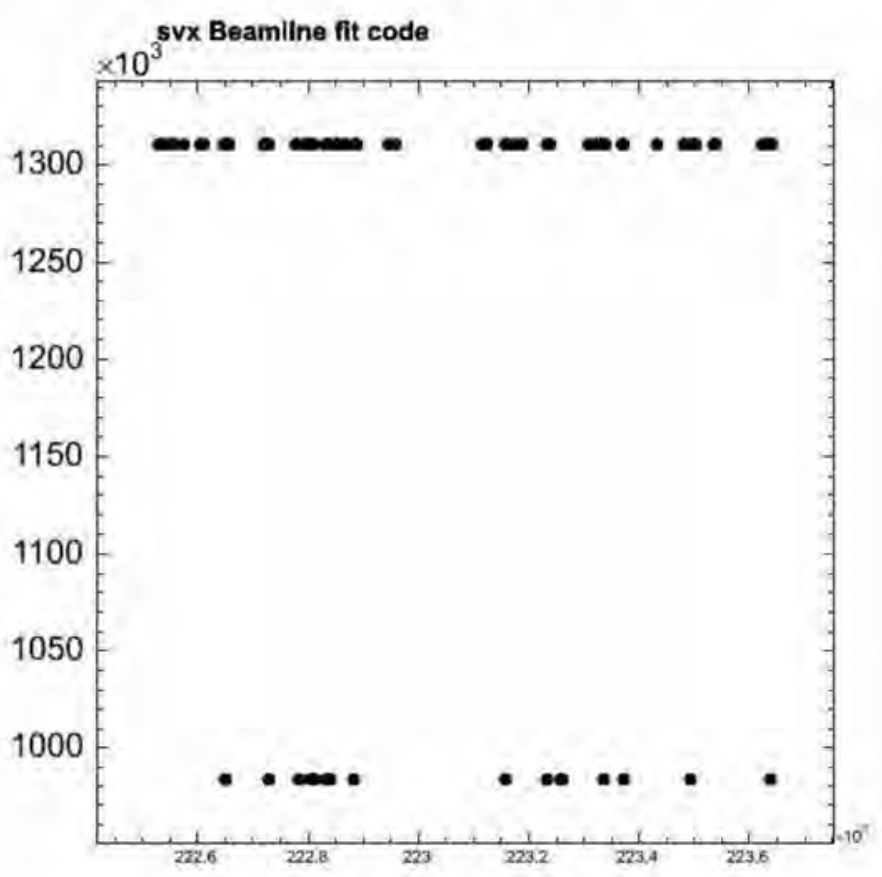

Figure A.3. Fit Codes for SVX with run 222427 223642. Two constant values are stored; lower number means low statistical run. 


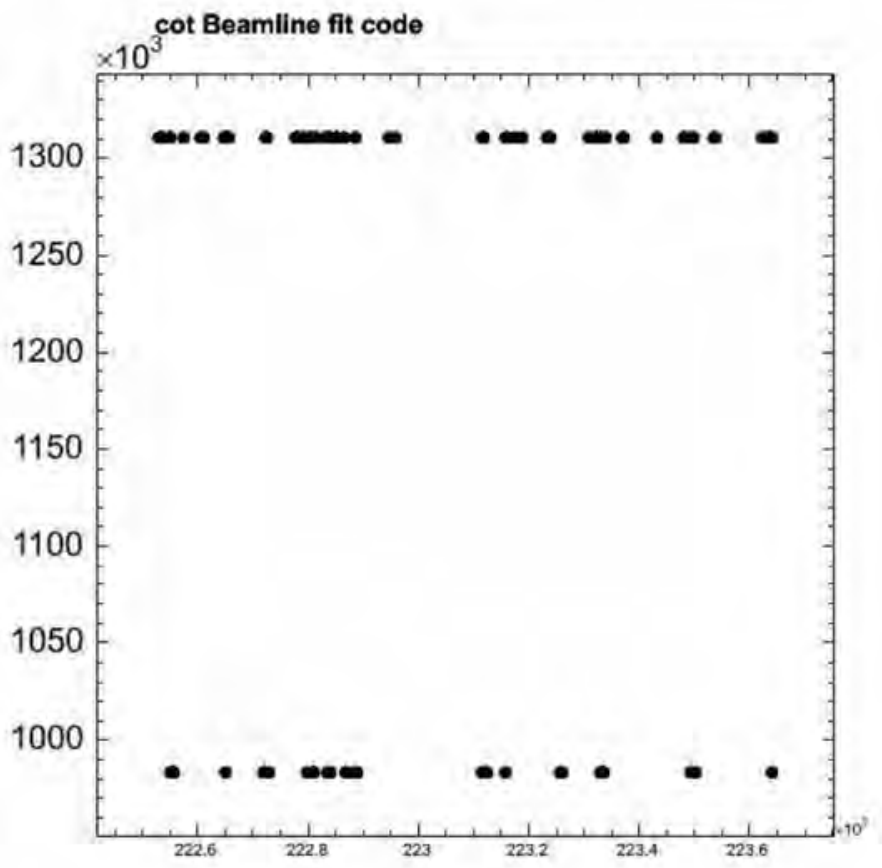

Figure A.4. Fit Codes for COT with run 222427 223642. Two constant values are stored; lower number means low statistical run.

112 

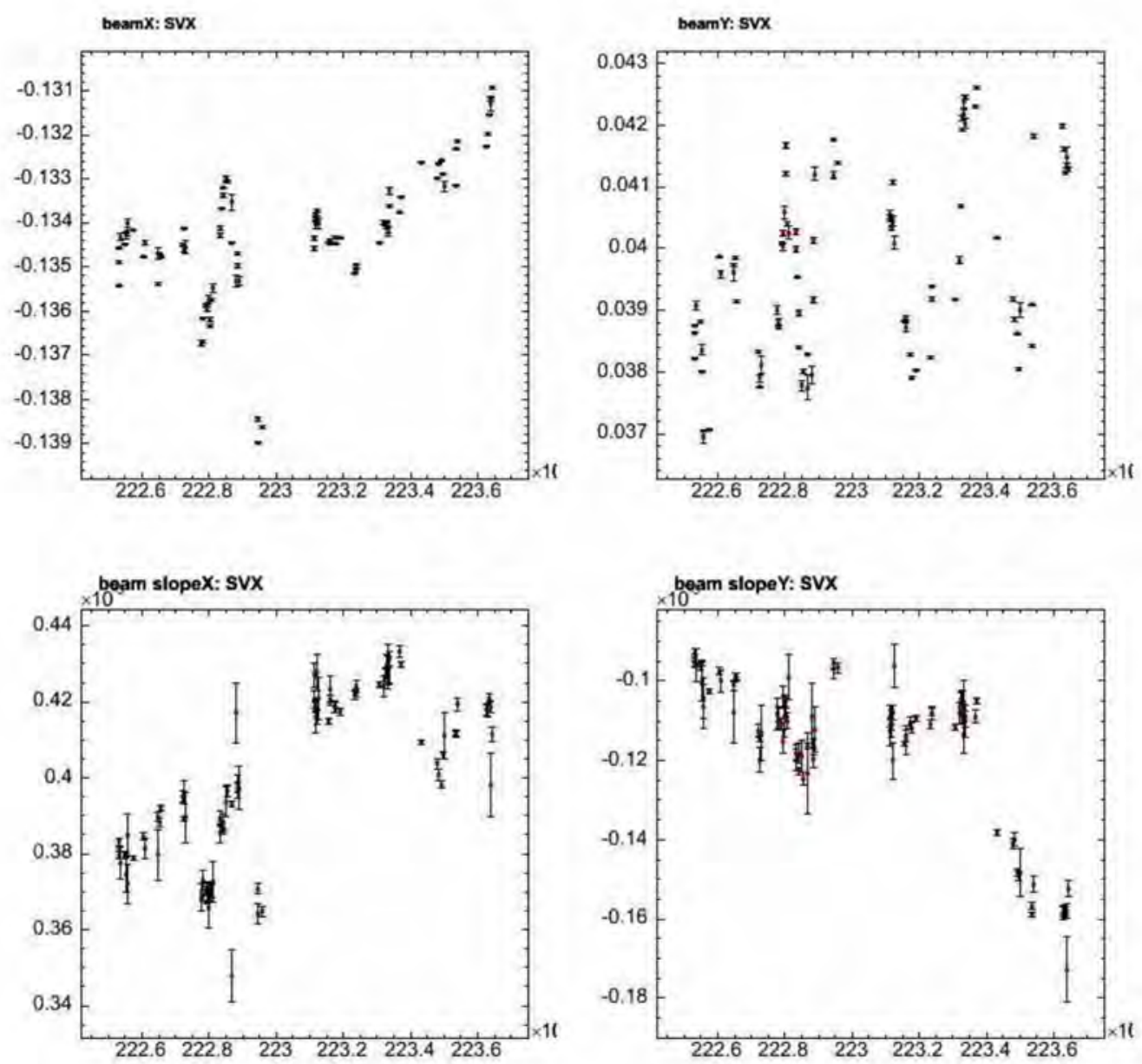

Figure A.5. BeamX Position of SVX with run 222427 223642 

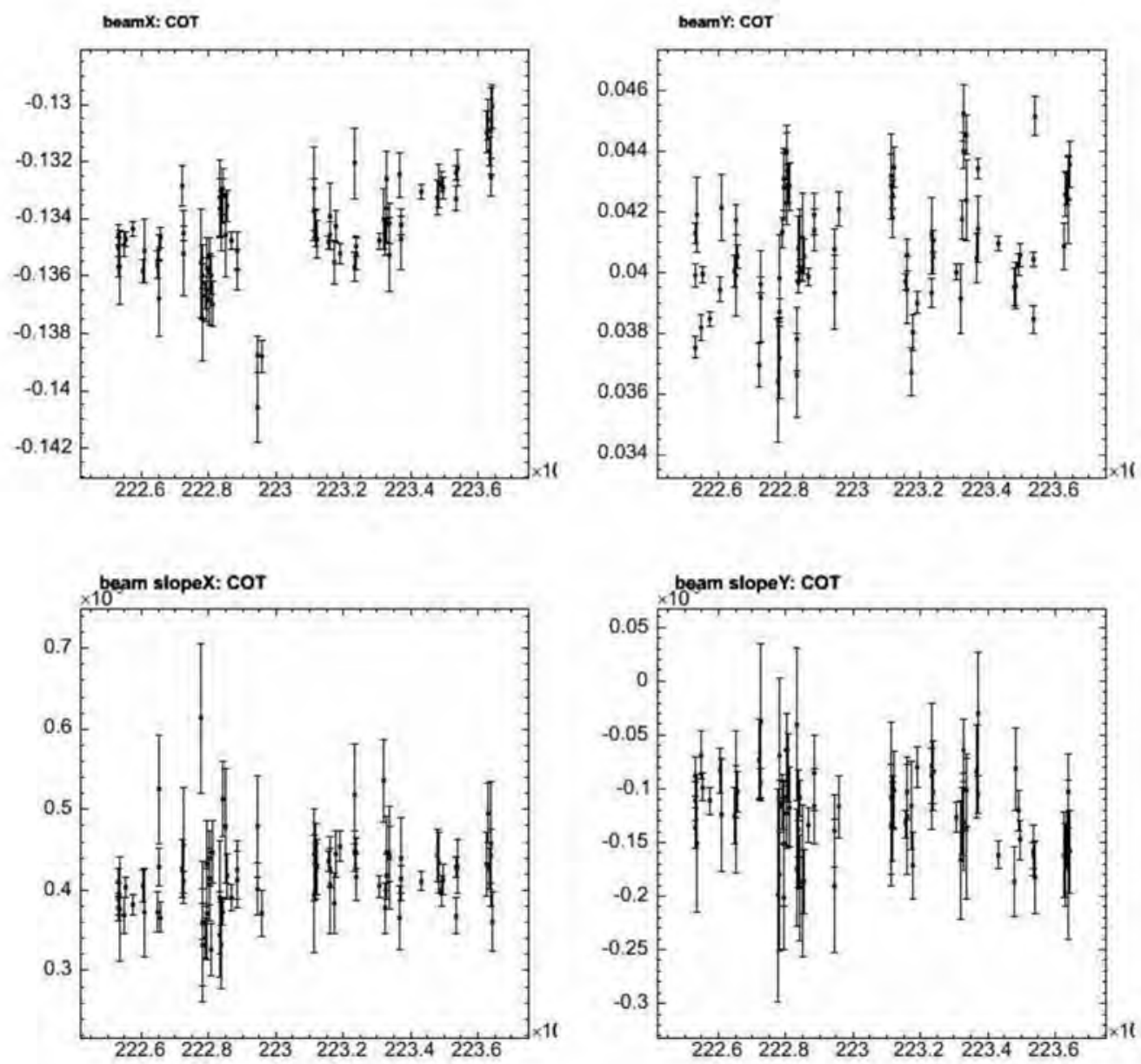

Figure A.6. BeamX Position of COT with run 222427 223642 

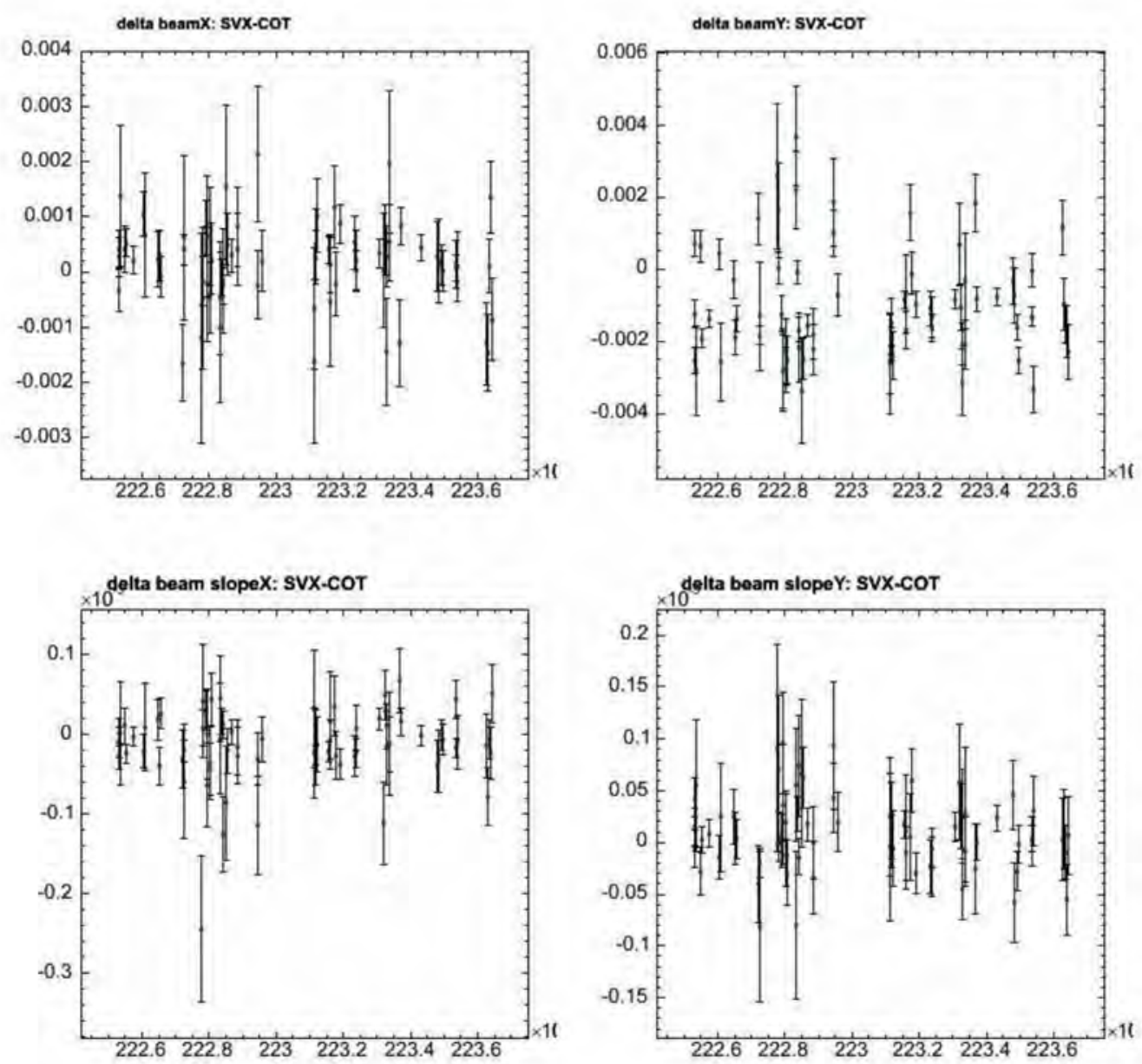

Figure A.7. Difference of SVX and COT beamline with run $222427 \sim 223642$ 

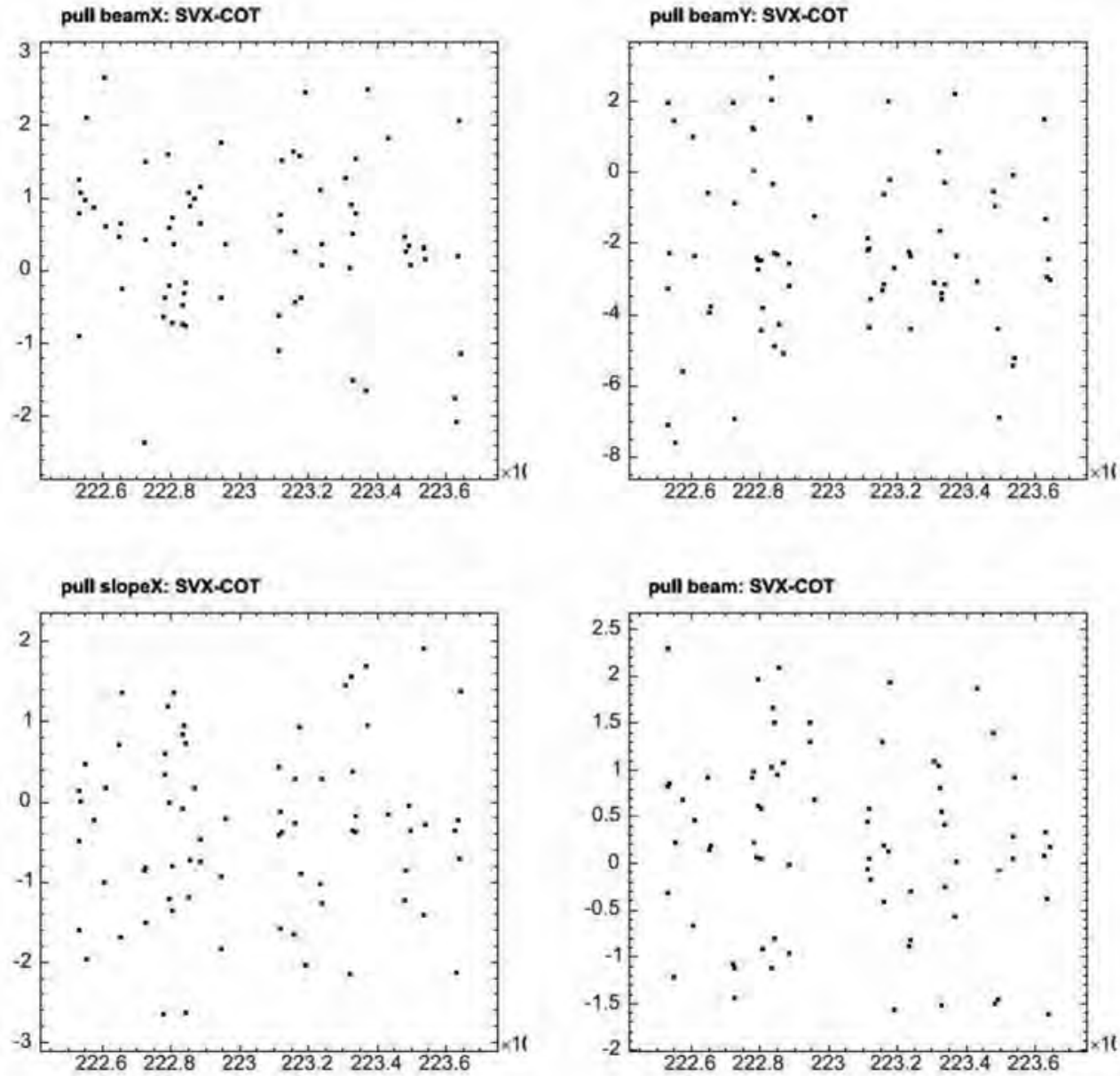

Figure A.8. Pulls of SVX-COT with run $222427 \sim 223642$ 

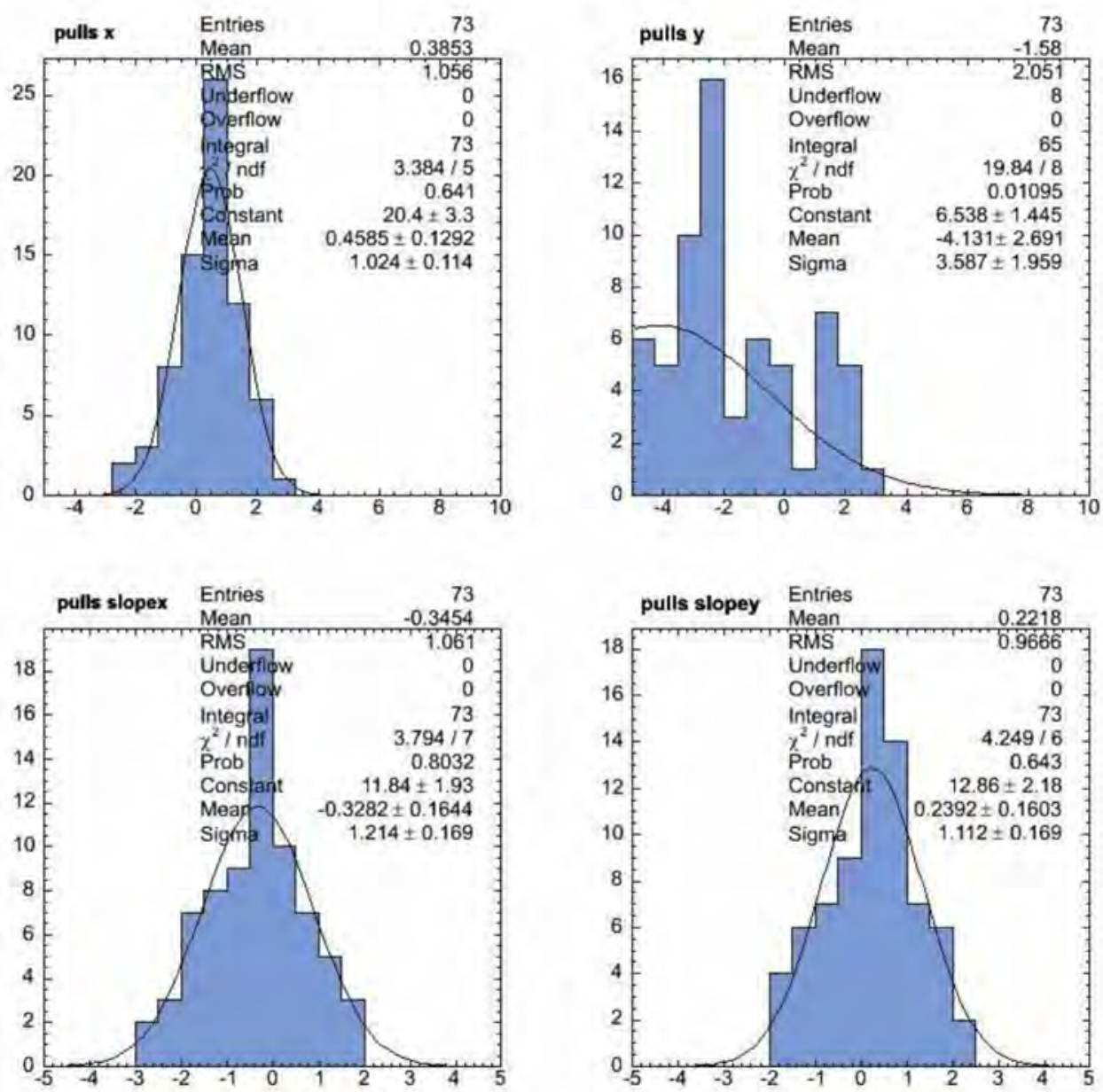

Figure A.9. Pull distribution of SVX-COT with run 222427 223642 


\section{Appendix B}

\section{Simulation of Single-top Production including NLO effect}

We present a comparison between the MC@NLO samples and the official MadEvent samples currently used by the single-top group. In general we find good agreement between the two samples, but there are small number of differences.

The next-to-reading oder(NLO) QCD corrections enlarge the leading-order cross section by about $30 \%$ at Tevatron. For the analysis of single-top production, NLO results are important to have a reliable estimate of the number of events expected. The MC@NLO allows to match cross section computed at NLO in QCD with an event generator.

The t-channel LO and NLO process matching is done "by hand" in MadEvent. The two processes are generated separately, then the events from the two samples are combined using a technique of smoothing the $\mathrm{P}_{T}$ distribution of the second $\mathbf{b}$ et. On the other hand, the MC@NLO program has the LO and NLO processes mixed internally to reproduce the theoretical NLO prediction for the single-top production,

In the case of standard MC, a hard kinematic configuration is generated on an event-by-event basis, and it is subsequently showered and hadronized. In the case of MC@NLO. all of the hard kinematic configuration (events)are generated in advance, and stored in a file and then each event is showered and hadronized by HERWIG. We generated event files using MC@NLO 3.3 package. We tell HERWIG that the events should be read from the event file using Les Houches interface by specifying a negative value of the process code IPROC. For the singletop production, IPROC is $-2000-\mathrm{IC}\left(\mathrm{H}_{1} \mathrm{H}_{2} \rightarrow \mathbf{t} / \overline{\mathbf{t}}+\mathrm{X}\right),-2001-\mathrm{IC}\left(\mathrm{H}_{1} \mathrm{H}_{2} \rightarrow \mathbf{t}+\mathrm{X}\right)$, or $-2004-\mathrm{IC}\left(\mathrm{H}_{1} \mathrm{H}_{2} \rightarrow \mathbf{t}+\mathrm{X}\right)$ by setting $\mathrm{IC} / 10$ and $\mathrm{IC} / 20$ for s- and t-channel pro- 
duction, $\mathrm{H}_{1,2}$ represents hadrons, $\mathrm{p}$ or $\overline{\mathrm{p}}$.

We can set IL1CODE/ 1,2,3 for the lepton identification of W-decay. Spin correlation for the decay products are therefore taken into account.

\section{B.1 Generator Level}

The MadEvent samples and MC@NLO samples are compared at the hepg level. MadEvent single-top samples are LO s-channel, matched(LO+NLO) t-channel. and LO t-channel (for illustration purposes). The following pairs of samples are compared. (MadEvent LO s-channel, MC@NLO s-channel), (MadEvent LO tchannel, MC@NLO t-channel), and (MadEvent matched t-channel, MC@NLO t-channel). The compared plots of MadEvent and MC@NLO are shown in Figure B.1 B.9 ; the top mass and the eta, E, $\mathrm{P}_{t}, \mathrm{P}_{z}$ of lepton, neutrino, b-quark from top, top quark.

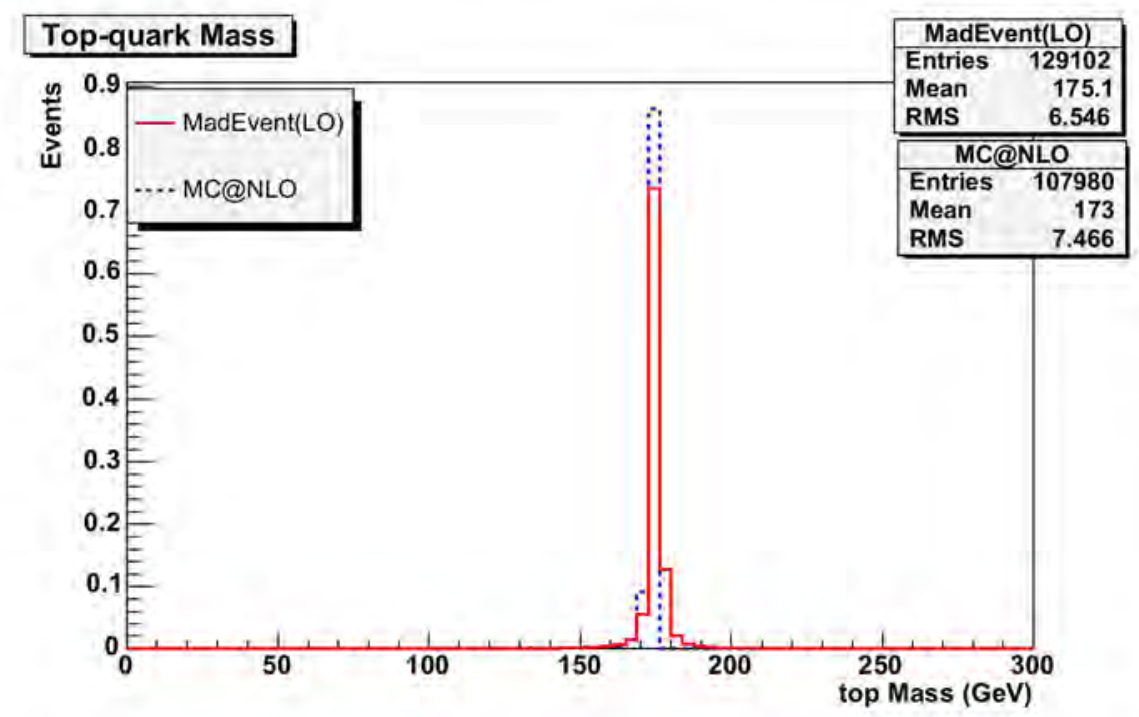

Figure B.1. s-channel sample. Top mass 

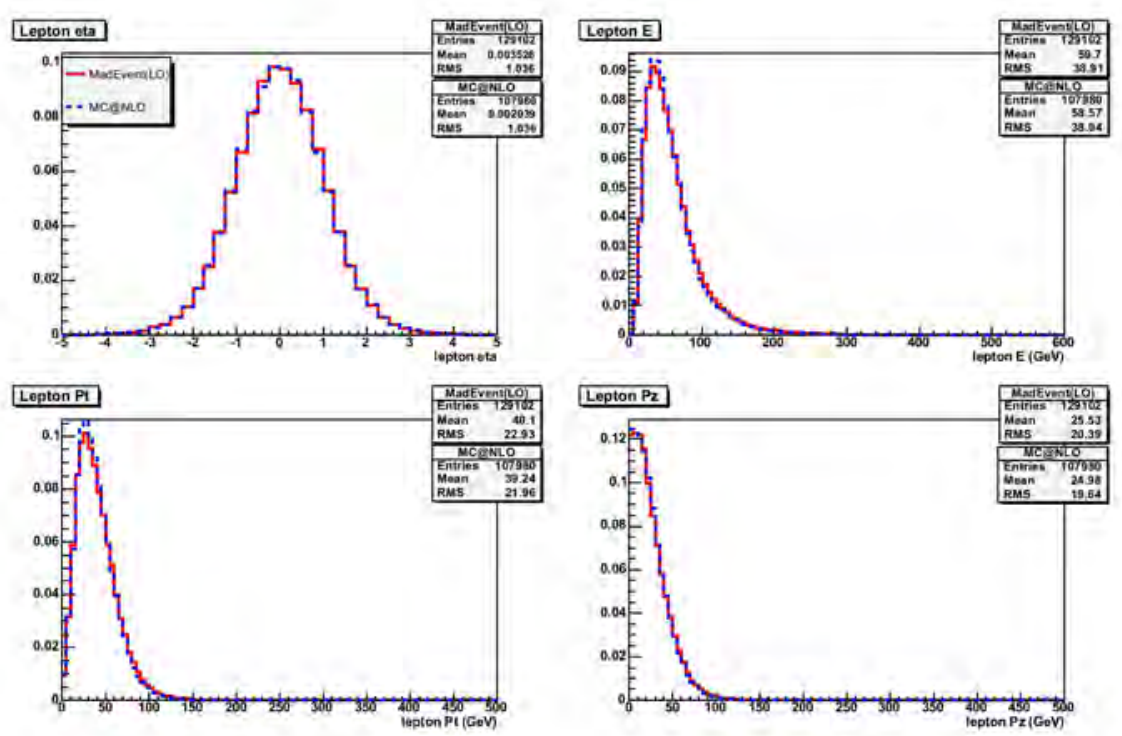

Figure B.2. s-channel sample. Lepton
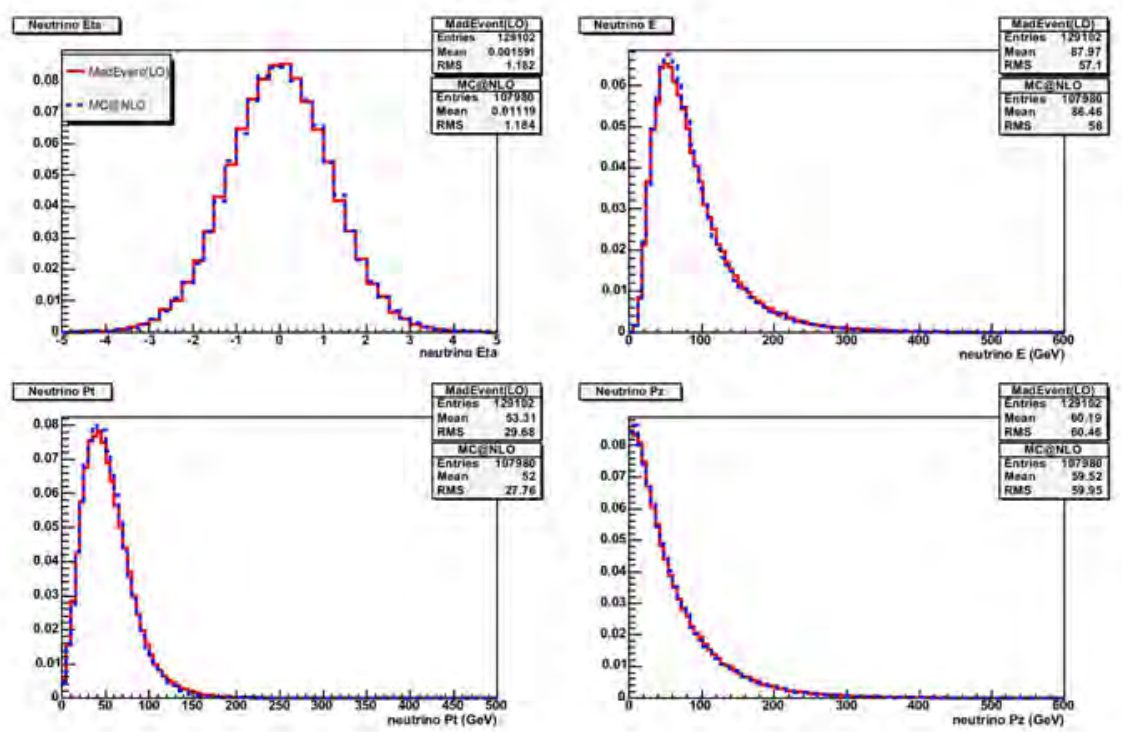

Figure B.3. s-channel sample. Neutrino 

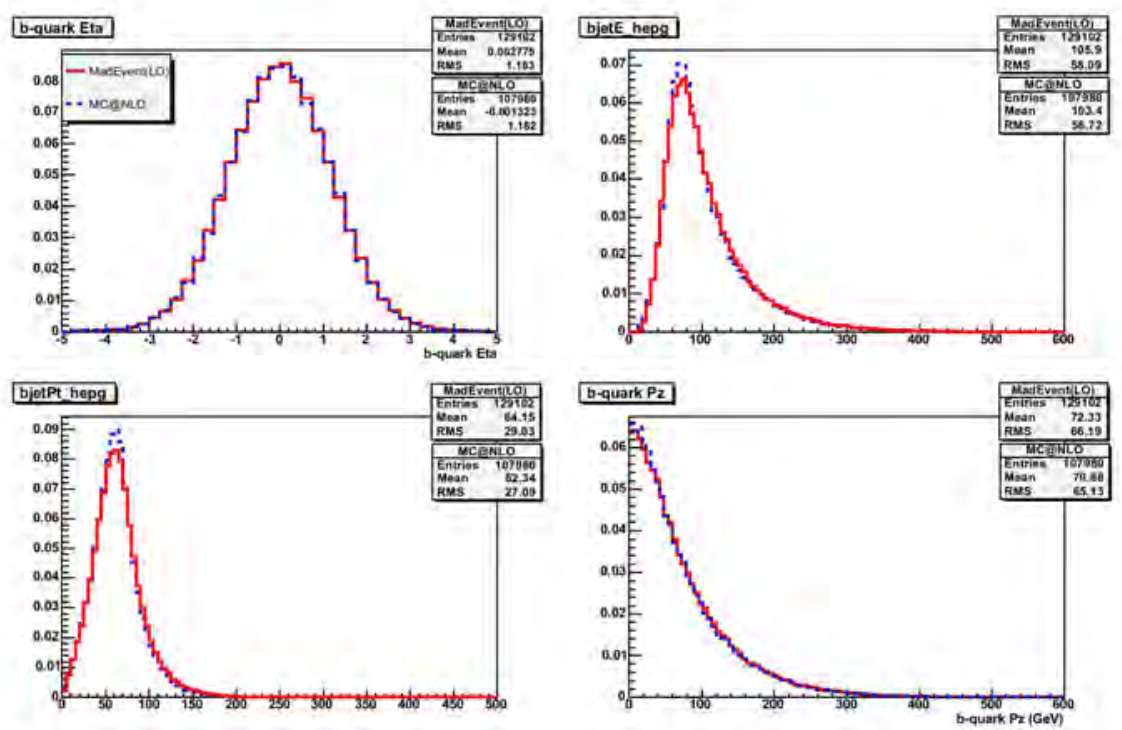

Figure B.4. s-channel sample. b-quark from top decay
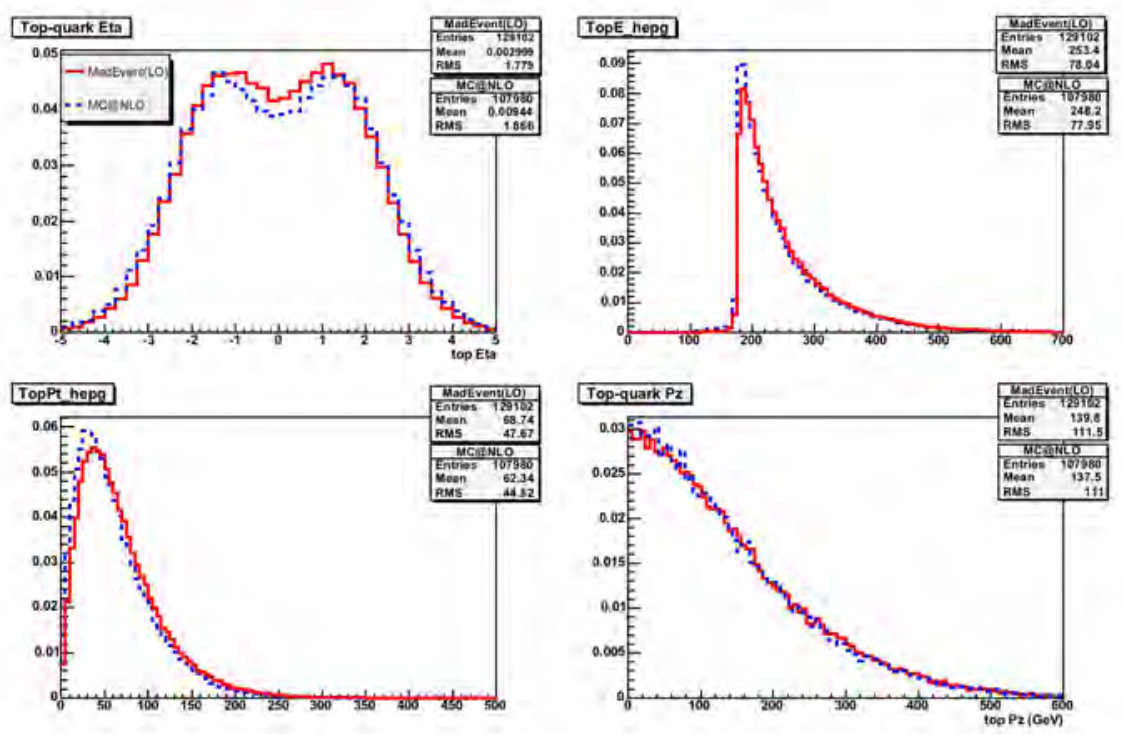

Figure B.5. s-channel sample. Top quark 


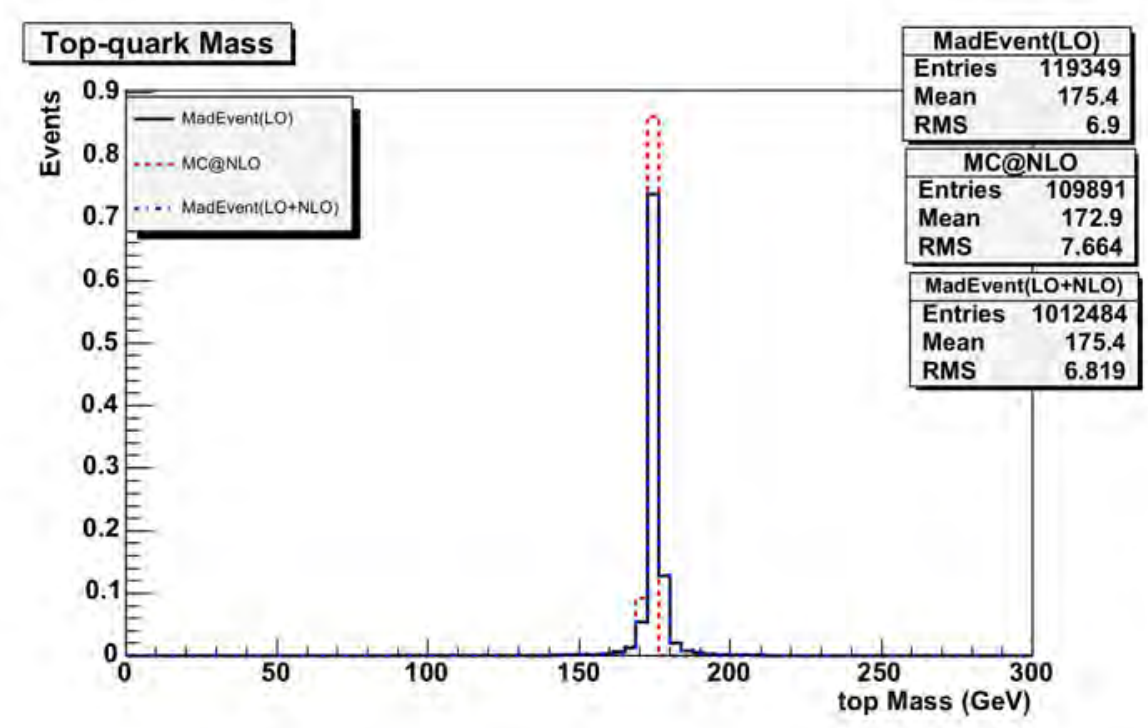

Figure B.6. t-channel sample. Top mass
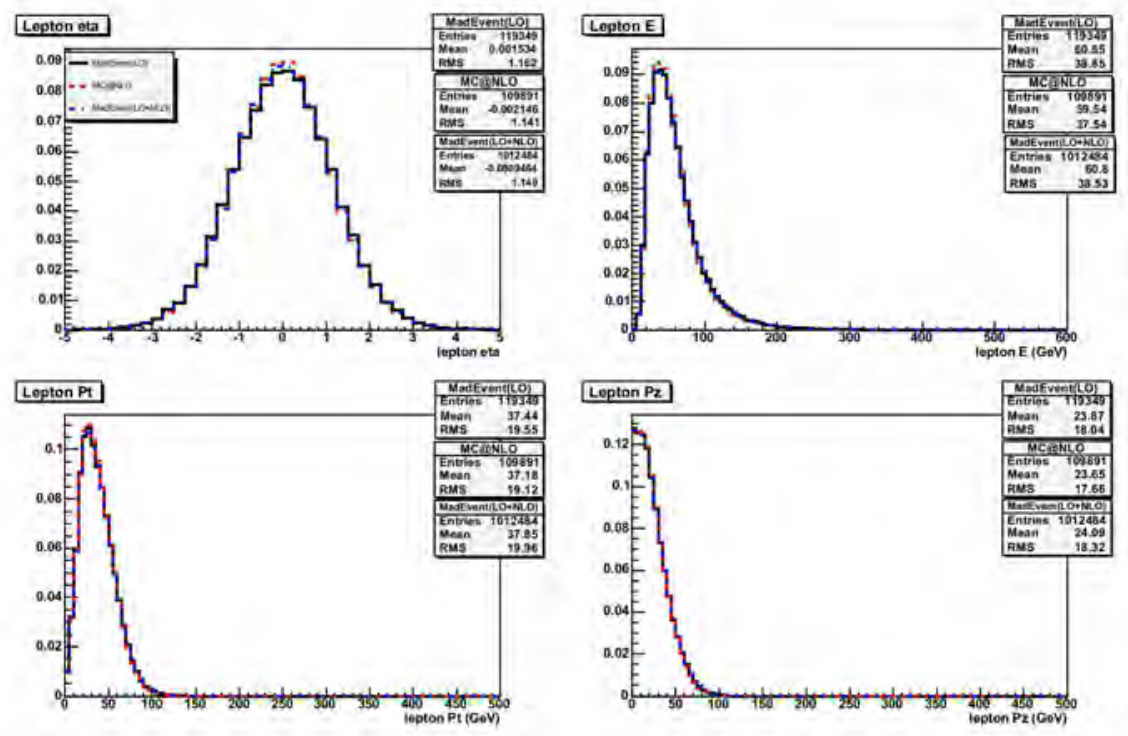

Figure B.7. t-channel sample. Lepton 

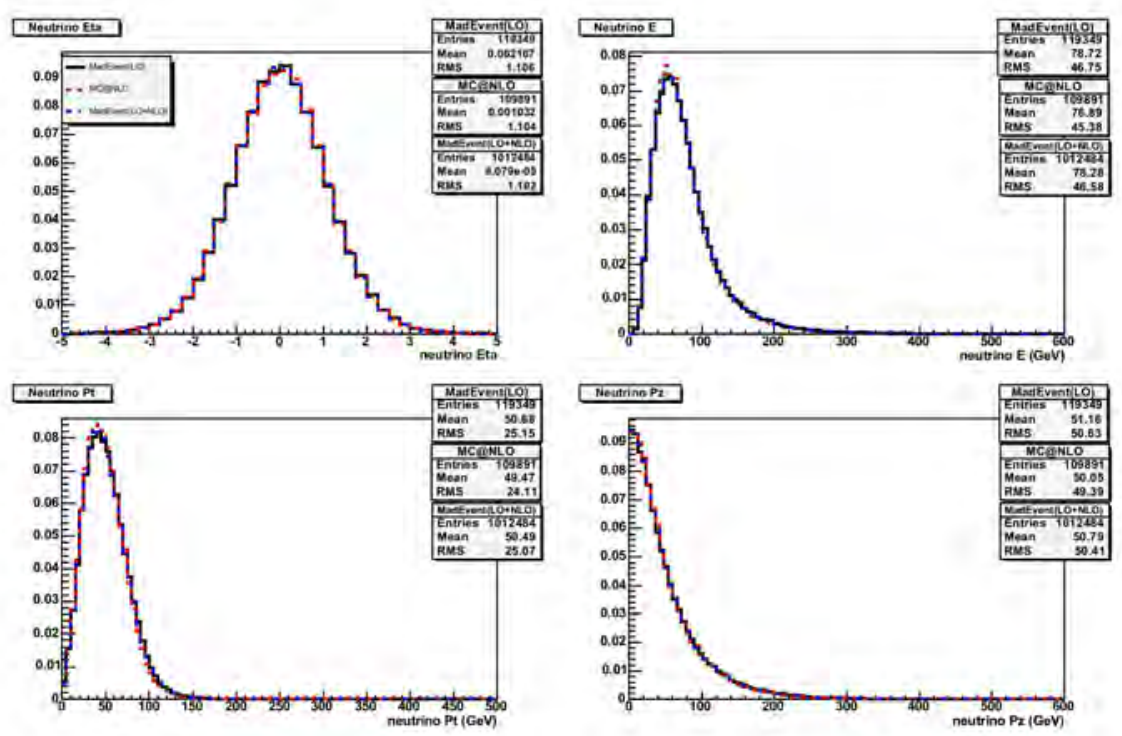

Figure B.8. t-channel sample. Neutrino
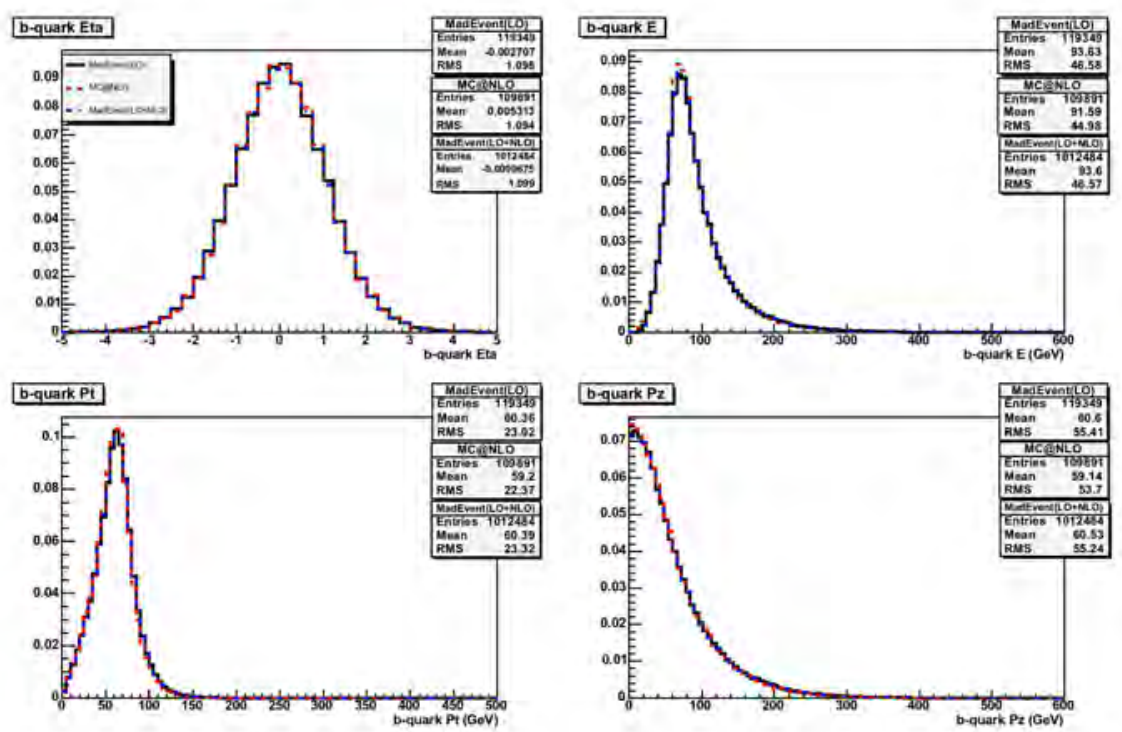

Figure B.9. t-channel sample. b-quark from top decay 


\section{B.2 Reconstruction level}

The comparison of the acceptances for the two generator samples is shown in Table B.1 B.2 B.3 B.4. First two rows show the numbers of events while the following rows show the fraction of events out of the "Good mu" total that pass the given sequence of cuts up to that point. For example, in Table 3, the number of 0Jet GoodRun has 4951 events and OJet Met has 405 events, which leads to the 8.18016 entry. If we look at the 2 - et channel, the official s-channel sample has a lower acceptance than MC@NLO (by 7\%). The 3-let bin however is more discrepant. For the t-channel samples, the 2-let bin agreement is excellent, while the overall acceptances (all let bins) are within 3-4\%.

The compared plots of MadEvent and MC@NLO are shown in Figure B.10 B.21 ; the top mass and the eta, E, $\mathrm{P}_{t}, \mathrm{P}_{z}$ of lepton, neutrino, b-quark from top, top quark.

\begin{tabular}{|c|c|c|c|c|c|c|}
\hline ALL & 0Jets & 1Jet & 2Jets & 3Jets & 4+Jets & Total \\
\hline Initial & 5308 & 73540 & 235560 & 149876 & 52312 & 516596 \\
\hline GoodRun & 4951 & 69092 & 220103 & 137426 & 47807 & 479379 \\
\hline \hline Trigger & 100 & 100 & 100 & 100 & 100 & 100 \\
\hline$>$ / 1TLep & 9.73540 & 9.45406 & 8.38925 & 4.84697 & 4.24624 & 7.12797 \\
\hline Met & 8.18016 & 7.84461 & 6.95356 & 4.05454 & 3.66055 & 5.93517 \\
\hline Iso & 8.07917 & 7.55948 & 6.53421 & 3.41638 & 2.738092 & 5.42556 \\
\hline Đilep & 7.95798 & 7.47119 & 6.48787 & 3.40110 & 2.72345 & 5.38446 \\
\hline Z & 7.89739 & 7.42343 & 6.45652 & 3.37563 & 2.70462 & 5.35338 \\
\hline Gosmic/Chi2 & 7.89739 & 7.40461 & 6.42199 & 3.20827 & 2.51009 & 5.26743 \\
\hline !DiffZ & 7.89739 & 7.40461 & 6.42153 & 3.20754 & 2.50590 & 5.26660 \\
\hline QCD & 7.89739 & 7.10791 & 6.04716 & 2.99142 & 2.50590 & 4.98997 \\
\hline SiGoodRun & 7.89739 & 7.10791 & 6.04716 & 2.99142 & 2.50590 & 4.98999 \\
\hline Taggable & 0 & 5.29583 & 5.54331 & 2.83206 & 2.42014 & 4.36168 \\
\hline$>/ 1+$ b-tag & 0 & 2.59798 & 3.42657 & 1.76240 & 1.51860 & 2.60441 \\
\hline
\end{tabular}

Table B.1. Acceptance table. s-channel MadEvent 


\begin{tabular}{|c|c|c|c|c|c|c|}
\hline ALL & 0Jets & 1Jet & 2Jets & 3Jets & 4+Jets & Total \\
\hline Initial & 8152 & 107816 & 287748 & 189116 & 76172 & 669004 \\
\hline GoodRun & 7751 & 102614 & 272697 & 176993 & 71001 & 631056 \\
\hline \hline Trigger & 100 & 100 & 100 & 100 & 100 & 100 \\
\hline$>/$ 1TLep & 10.12772 & 9.78813 & 8.72286 & 5.76576 & 4.55204 & 7.61469 \\
\hline Met & 8.51503 & 8.05055 & 7.25640 & 4.84764 & 3.91966 & 6.34999 \\
\hline Iso & 8.38601 & 7.80010 & 6.79215 & 4.18773 & 3.02390 & 5.82119 \\
\hline Đilep & 8.24409 & 7.74748 & 6.73824 & 4.15779 & 2.98869 & 5.77524 \\
\hline$\not{Z}$ & 8.19249 & 7.70070 & 6.70121 & 4.13406 & 2.96052 & 5.74117 \\
\hline Gonv & 8.19249 & 7.69193 & 6.65867 & 4.00411 & 2.78446 & 5.66510 \\
\hline !DiffZ & 8.17958 & 7.69095 & 6.65793 & 4.00298 & 2.78446 & 5.66415 \\
\hline QCD & 8.17958 & 7.45414 & 6.32130 & 3.77246 & 2.78446 & 5.41552 \\
\hline SiGoodRun & 8.17958 & 7.45414 & 6.32130 & 3.77246 & 2.78446 & 5.41552 \\
\hline Taggable & 0 & 5.63665 & 5.88932 & 3.61087 & 2.69432 & 4.77738 \\
\hline$>$ / 1+b-tag & 0 & 2.63609 & 3.67294 & 2.24302 & 1.67462 & 2.83334 \\
\hline
\end{tabular}

Table B.2. Acceptance table. s-channel MC@NLO

\begin{tabular}{|c|c|c|c|c|c|c|}
\hline ALL & 0Jets & 1Jet & 2Jets & 3Jets & 4+Jets & Total \\
\hline Initial & 55468 & 711584 & 1830872 & 999340 & 273284 & 3870548 \\
\hline GoodRun & 52430 & 674338 & 1731507 & 931296 & 254225 & 3643796 \\
\hline \hline Trigger & 100 & 100 & 100 & 100 & 100 & 100 \\
\hline$>$ / 1TLep & 9.24661 & 8.98970 & 8.01590 & 4.30453 & 3.81119 & 6.97190 \\
\hline Met & 7.80278 & 7.41571 & 6.65044 & 3.61925 & 3.18497 & 5.79214 \\
\hline Iso & 7.68453 & 7.21492 & 6.34274 & 3.08709 & 2.46002 & 5.42047 \\
\hline Đilep & 7.57009 & 7.15650 & 6.31184 & 3.06862 & 2.44350 & 5.38745 \\
\hline$\not$ & 7.52431 & 7.10148 & 6.24548 & 3.03329 & 2.41360 & 5.33397 \\
\hline Gosmic/Chi2 & 7.52431 & 7.09065 & 6.20523 & 2.85140 & 2.20237 & 5.25161 \\
\hline !DiffZ & 7.52431 & 7.08947 & 6.20459 & 2.85075 & 2.20119 & 5.25084 \\
\hline QCD & 7.52431 & 6.86020 & 5.92085 & 2.70515 & 2.20119 & 5.03636 \\
\hline SiGoodRum & 7.52431 & 6.86020 & 5.92085 & 2.70515 & 2.20119 & 5.03636 \\
\hline Taggable & 0 & 4.88864 & 5.19558 & 2.53829 & 2.12842 & 4.17087 \\
\hline$>/ 1+b-t a g$ & 0 & 2.13023 & 2.40518 & 1.32095 & 1.26856 & 1.96328 \\
\hline
\end{tabular}

Table B.3. Acceptance table. t-chamel MadEvent 

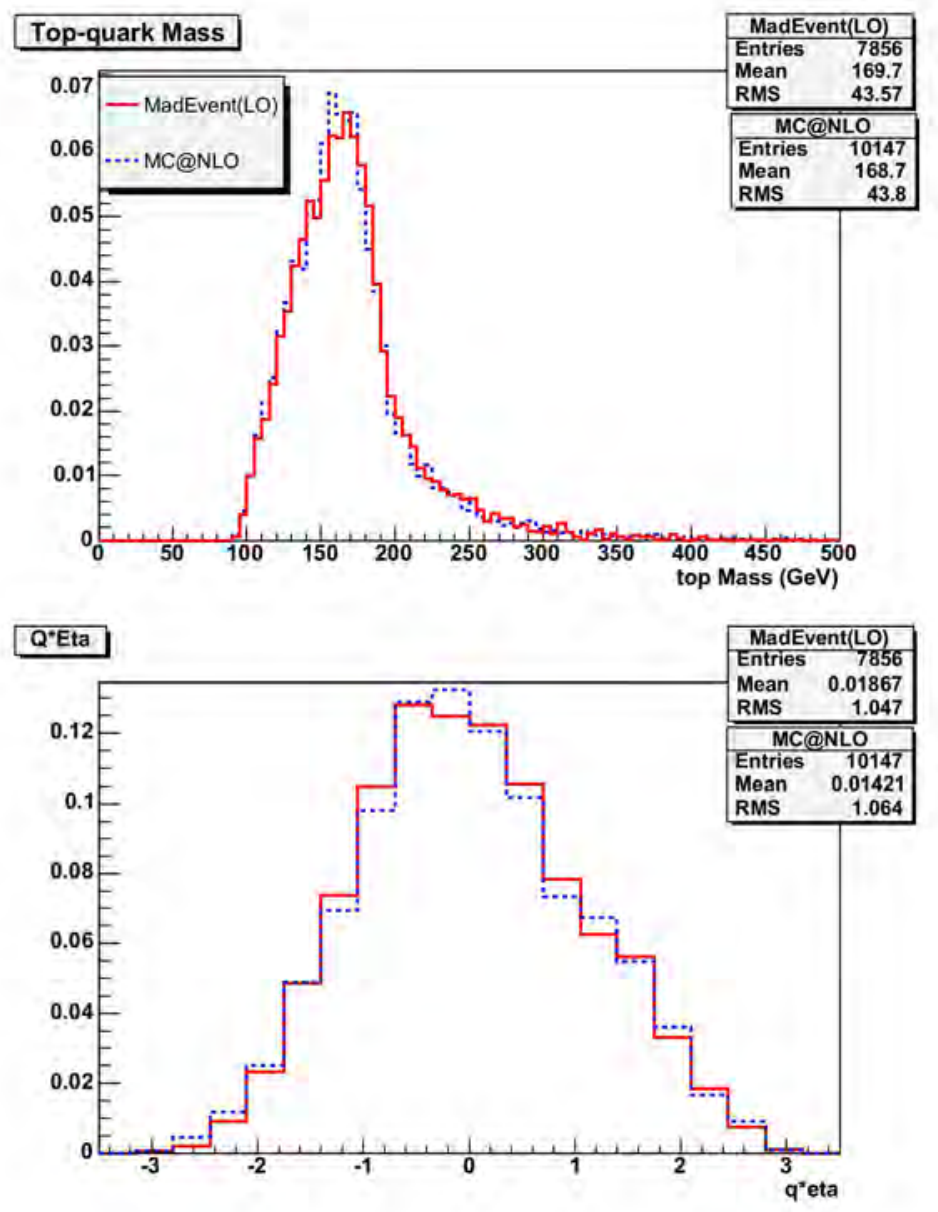

Figure B.10. s-channel sample. Top mass and $\mathrm{Q} \times \eta$ 


\begin{tabular}{|c|c|c|c|c|c|c|}
\hline ALL & 0Jets & 1Jet & 2Jets & 3Jets & 4+Jets & Total \\
\hline Initial & 10504 & 130488 & 308572 & 173028 & 49708 & 672300 \\
\hline GoodRun & 9930 & 123593 & 291755 & 161613 & 46204 & 633095 \\
\hline \hline Trigger & 100 & 100 & 100 & 100 & 100 & 100 \\
\hline$>$ / 1TLep & 9.67774 & 9.31201 & 8.26960 & 4.86594 & 3.68582 & 7.29179 \\
\hline Met & 8.39879 & 7.69218 & 6.88077 & 4.08073 & 3.17072 & 6.07744 \\
\hline Iso & 8.33836 & 7.54168 & 6.60759 & 3.62408 & 2.53874 & 5.75853 \\
\hline Đilep & 8.17724 & 7.46239 & 6.56304 & 3.60985 & 2.52142 & 5.71509 \\
\hline$Z$ & 8.11681 & 7.40090 & 6.46569 & 3.5516 & 2.47814 & 5.63928 \\
\hline Gonv & 8.11681 & 7.39362 & 6.42936 & 3.40628 & 2.31798 & 5.57230 \\
\hline !DiffZ & 8.10674 & 7.39281 & 6.42833 & 3.40566 & 2.31798 & 5.57135 \\
\hline QCD & 8.10674 & 7.16464 & 6.14385 & 3.23860 & 2.31798 & 5.35306 \\
\hline SiGoodRun & 8.10674 & 7.16464 & 6.14385 & 3.23860 & 2.31798 & 5.35306 \\
\hline Taggable & 0 & 5.09009 & 5.33941 & 3.03193 & 2.19677 & 4.38859 \\
\hline$>$ / 1+b-tag & 0 & 2.20562 & 2.43491 & 1.52215 & 1.21850 & 2.03018 \\
\hline
\end{tabular}

Table B.4. Acceptance table. t-channel MC@NLO
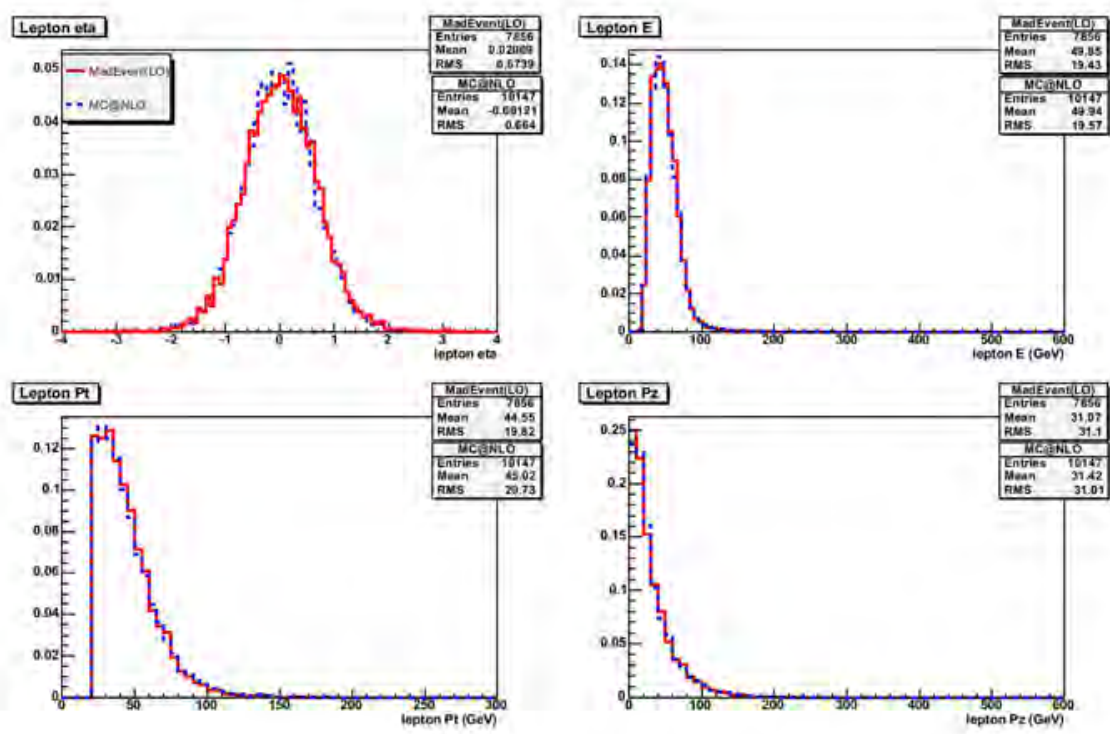

Figure B.11. s-channel sample, Lepton 

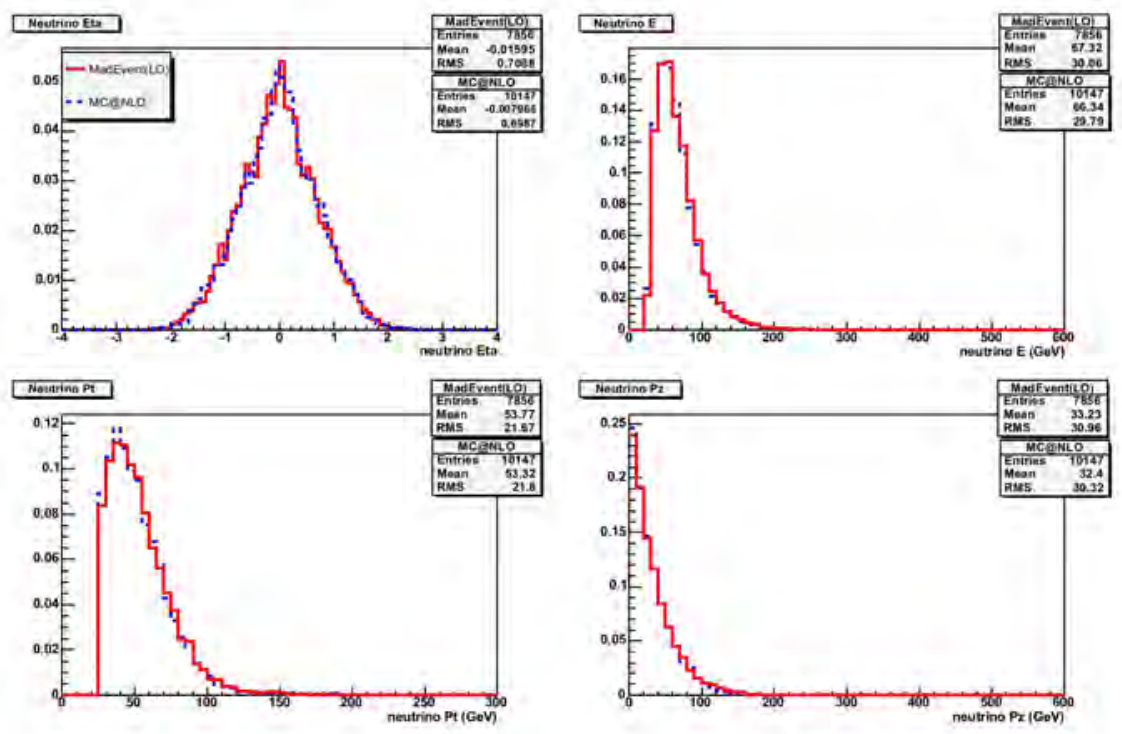

Figure B.12. s-channel sample. Neutrino
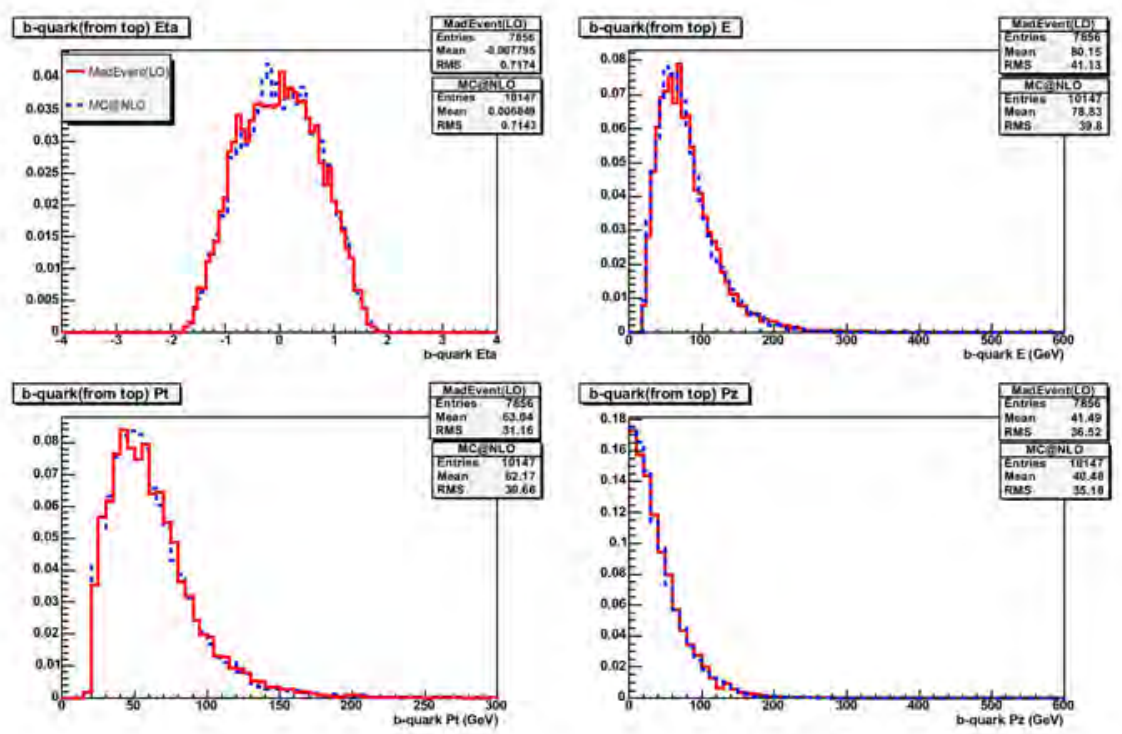

Figure B.13. s-channel sample. b-quark from top decay 

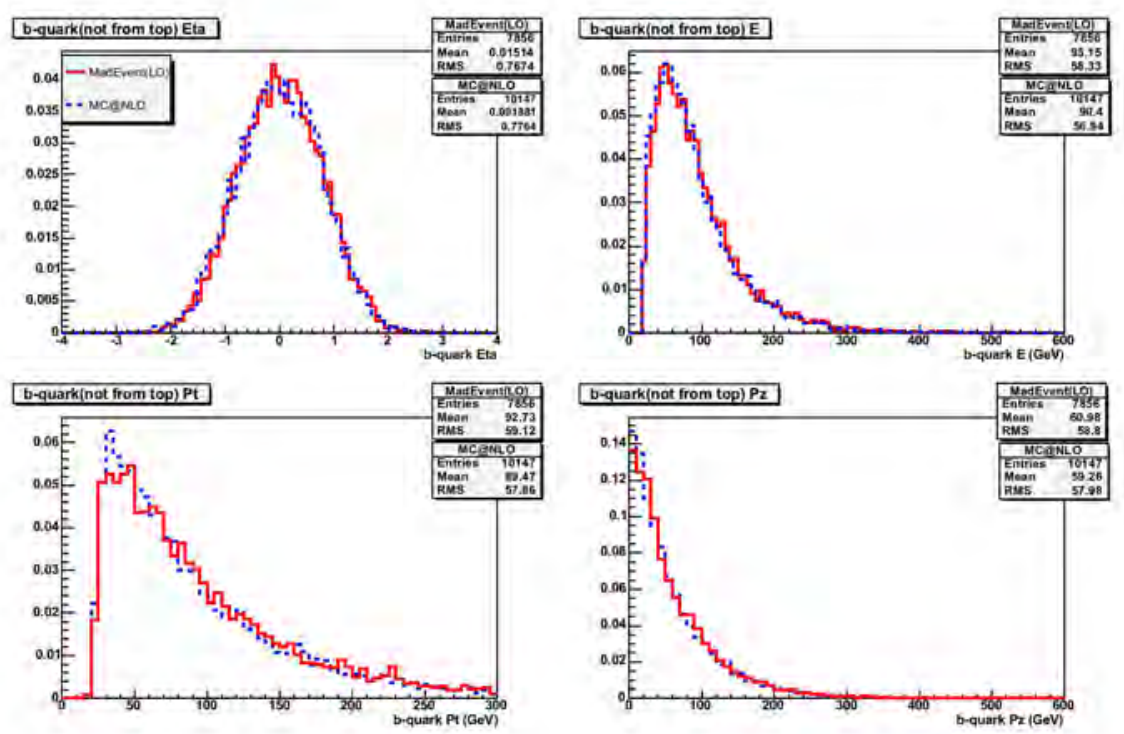

Figure B.14. s-channel sample. b-quark not from top decay
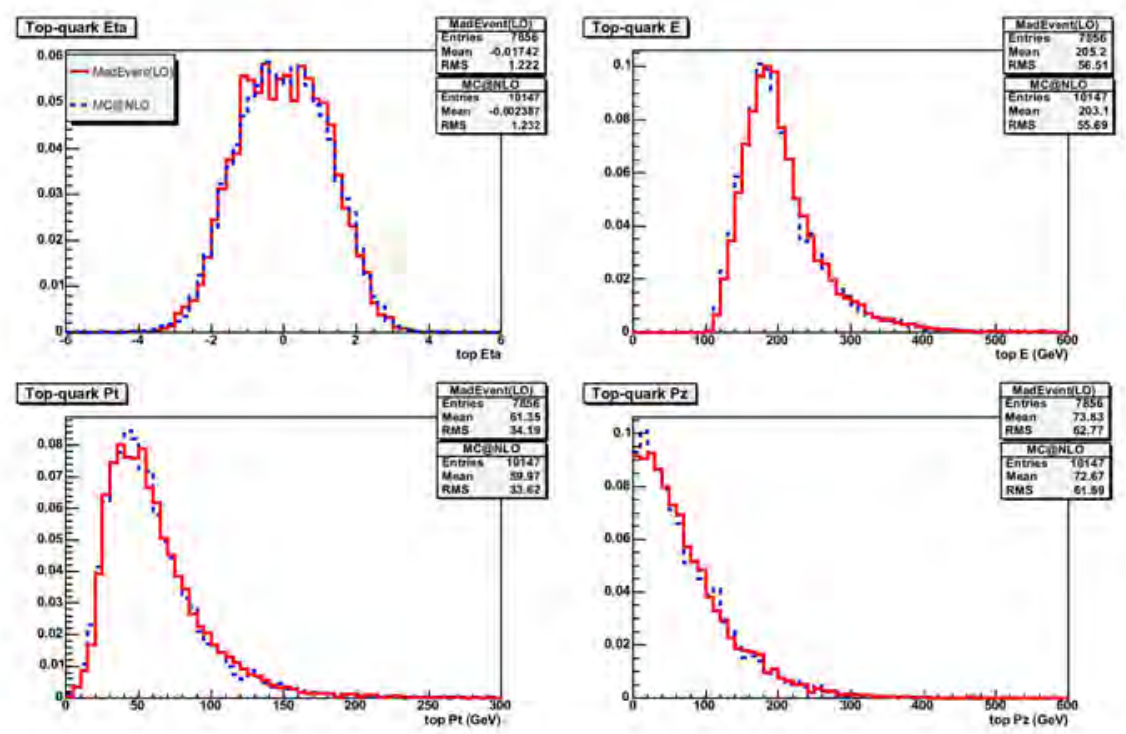

Figure B.15. s-channel sample. Top quark 


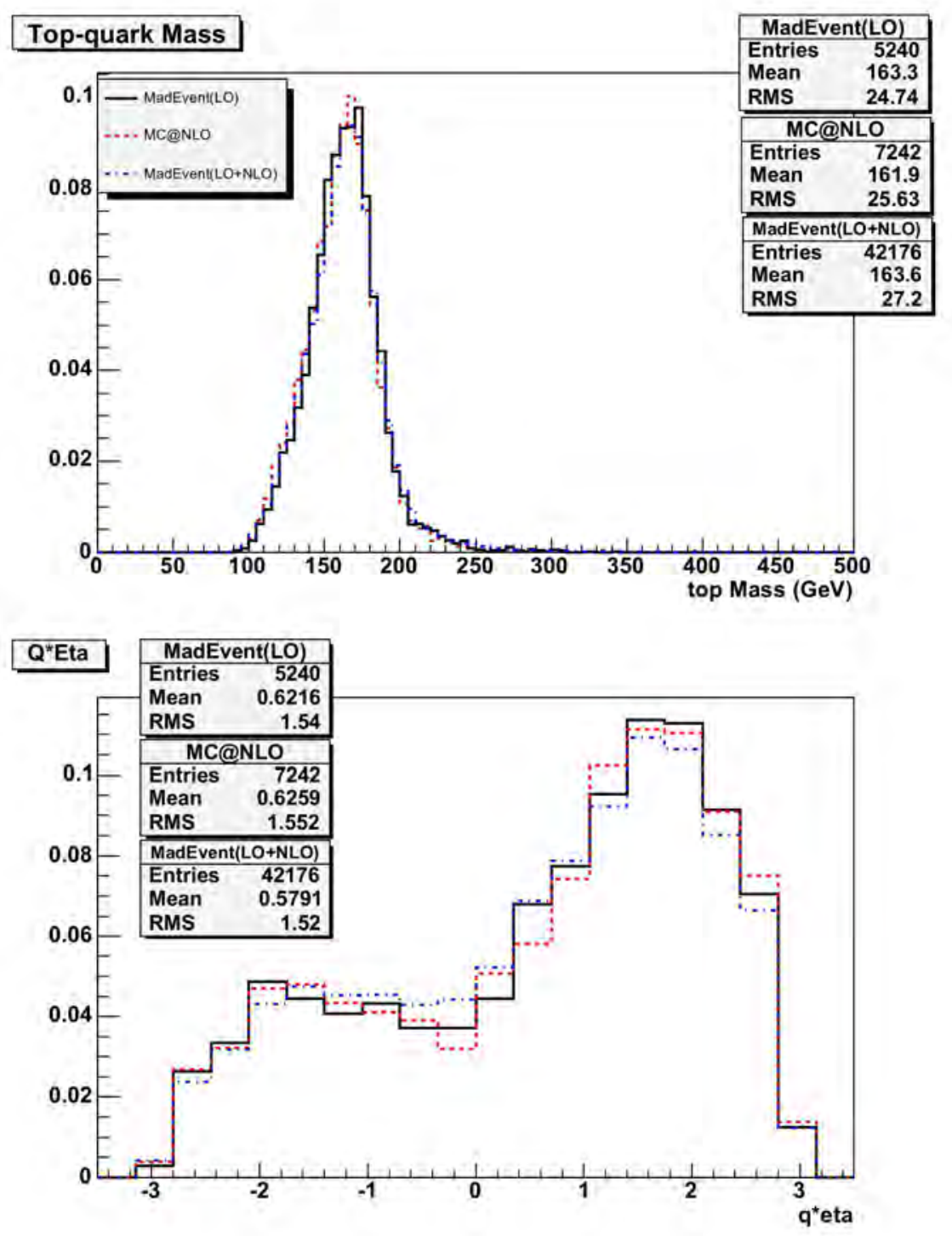

Figure B.16. t-channel sample. Top mass and $Q \times \Pi$ 

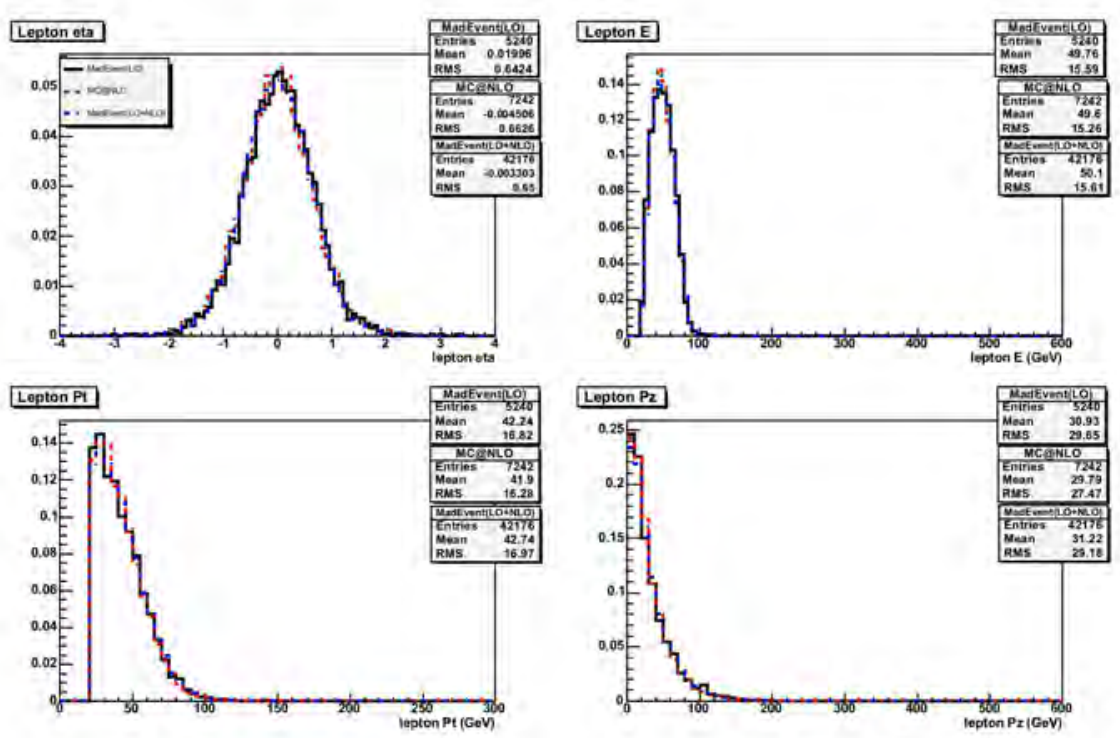

Figure B.17. t-channel sample. Lepton
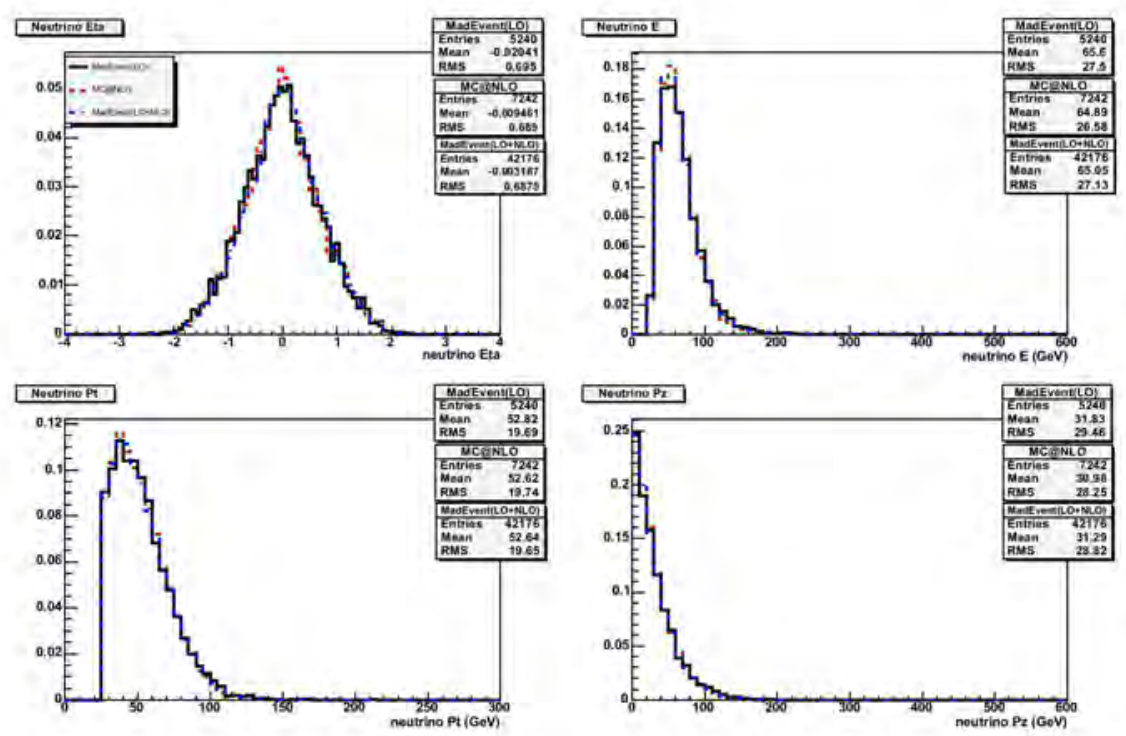

Figure B.18. t-channel sample. Neutrino 

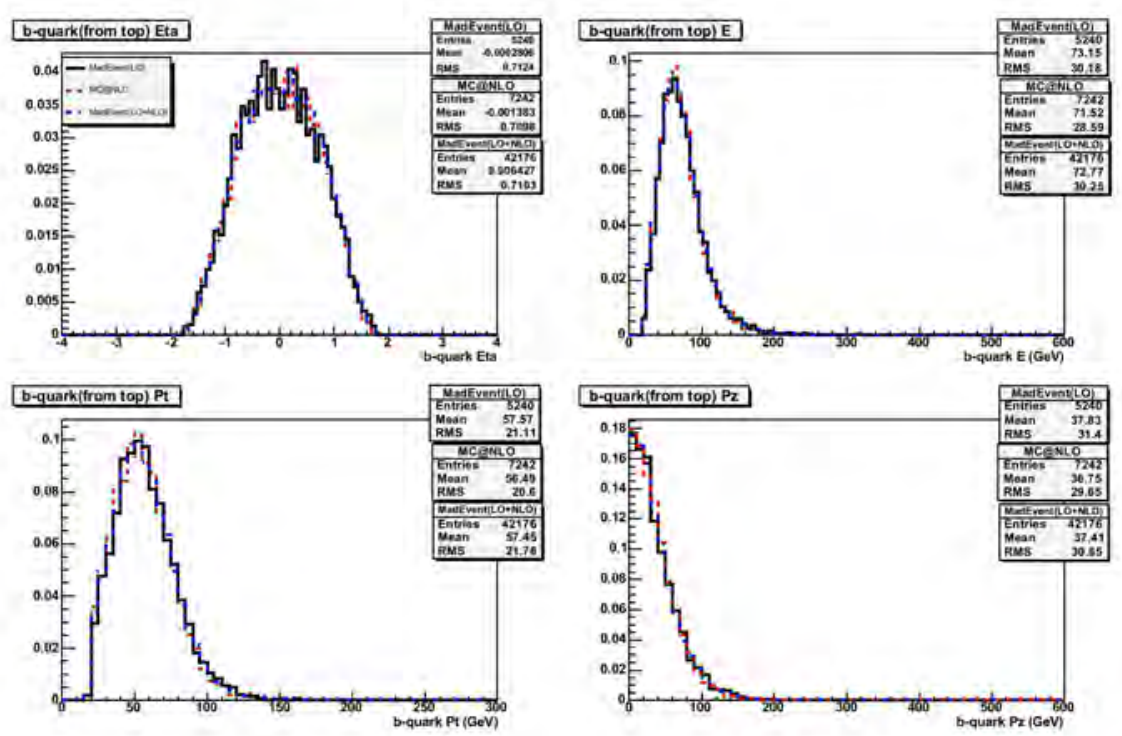

Figure B.19. t-channel sample. b-quark from top decay
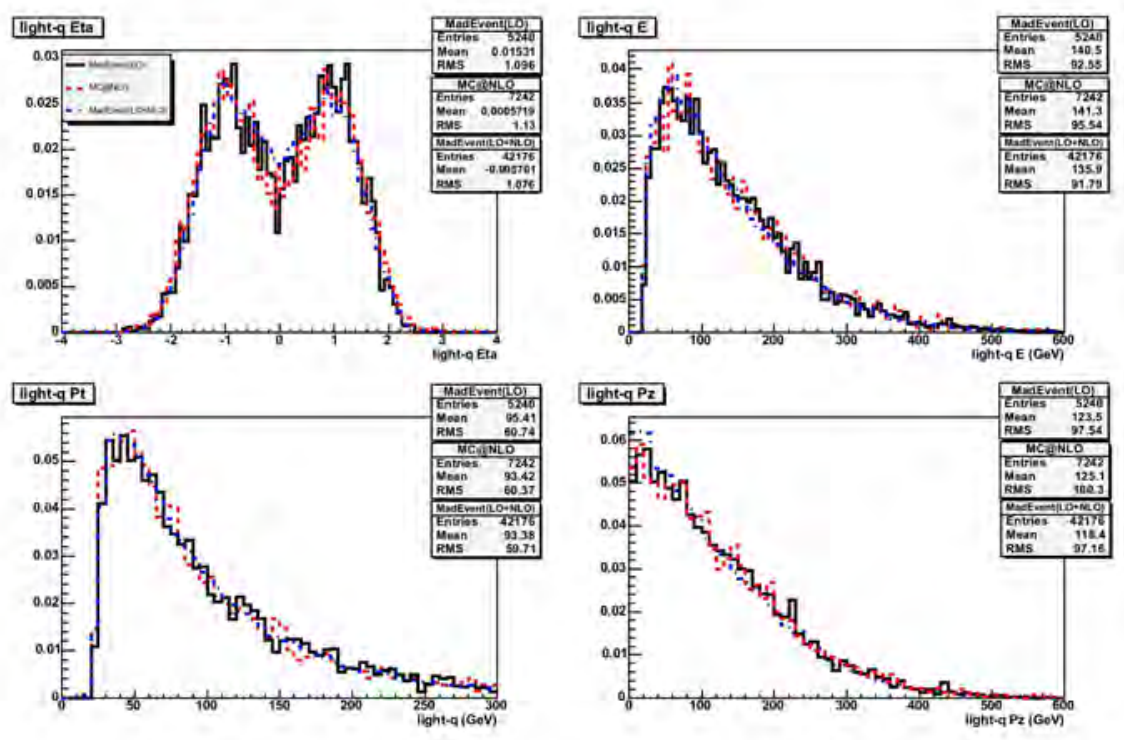

Figure B.20. t-channel sample. Light quark 

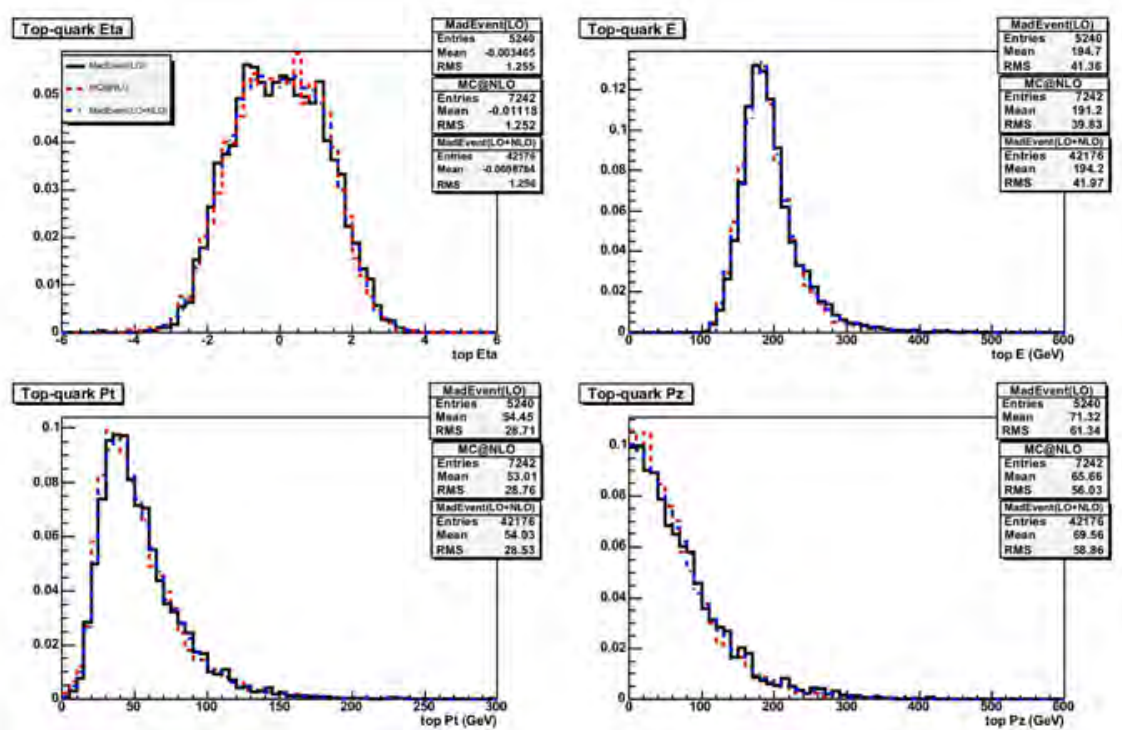

Figure B.21. t-channel sample. Top quark 


\section{Appendix C}

\section{Shape Systematic Uncertainty}

\section{C.1 Plots}

Compared with rate uncertainties, shape systematic uncertainties are considered as shape distortions of the templates for the signals and backgrounds. All shape uncertainties are median smoothed with five bins except the first and last two bins. The shape systematic sources are JES, PDF, ISR/FSR, Jet Flavor Separator Modeling, Mistag Model, Non-W Flavor Composition, Jet $\eta$ Distribution, Jet $4 \mathrm{R}_{j j}$ Distribution. Figure C.1 C.18 show compared plot(up) and ratio plot(down) between central and systematic sample.
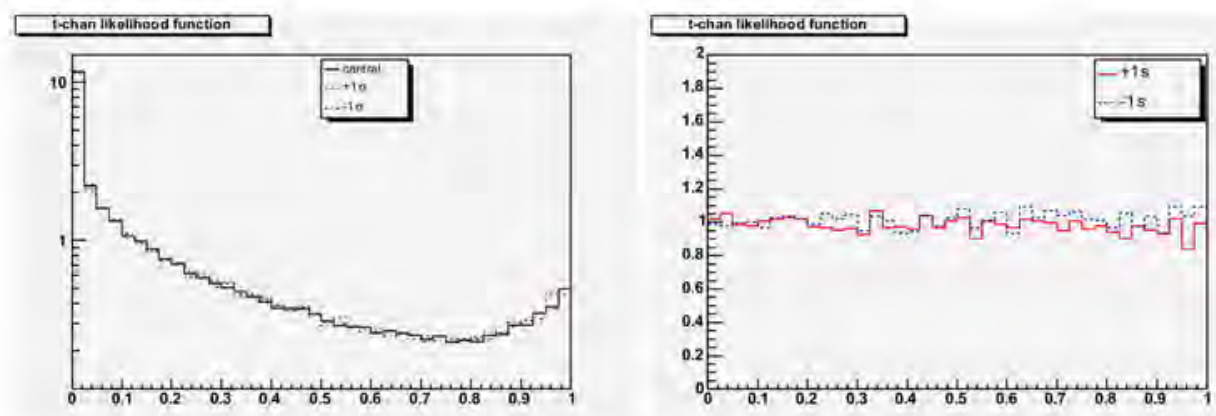

Figure C.1. JES Shape Systematic Uncertainty(s-channel); compared plot(up) and ratio plot(down) between central and systematic sample 

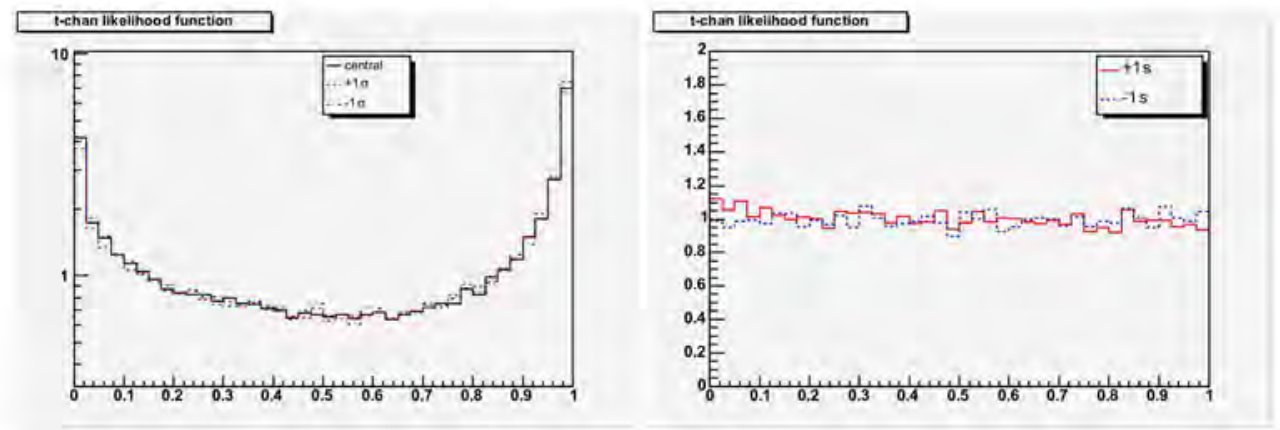

Figure C.2. JES Shape Systematic Uncertainty(t-channel); compared plot(up) and ratio plot(down) between central and systematic sample
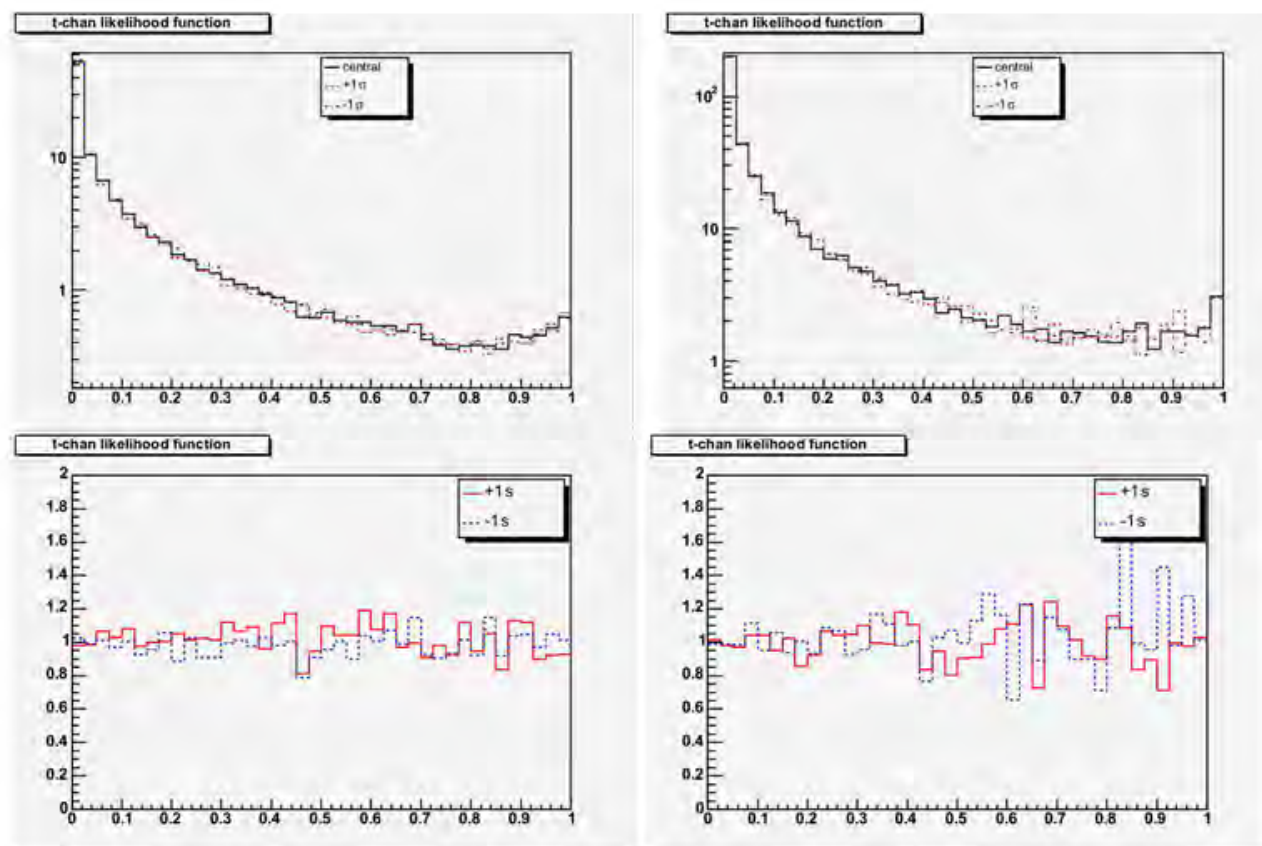

Figure C.3. JES Shape Systematic Uncertainty(ttbar/Wbb); compared plot(up) and ratio plot(down) between central and systematic sample 

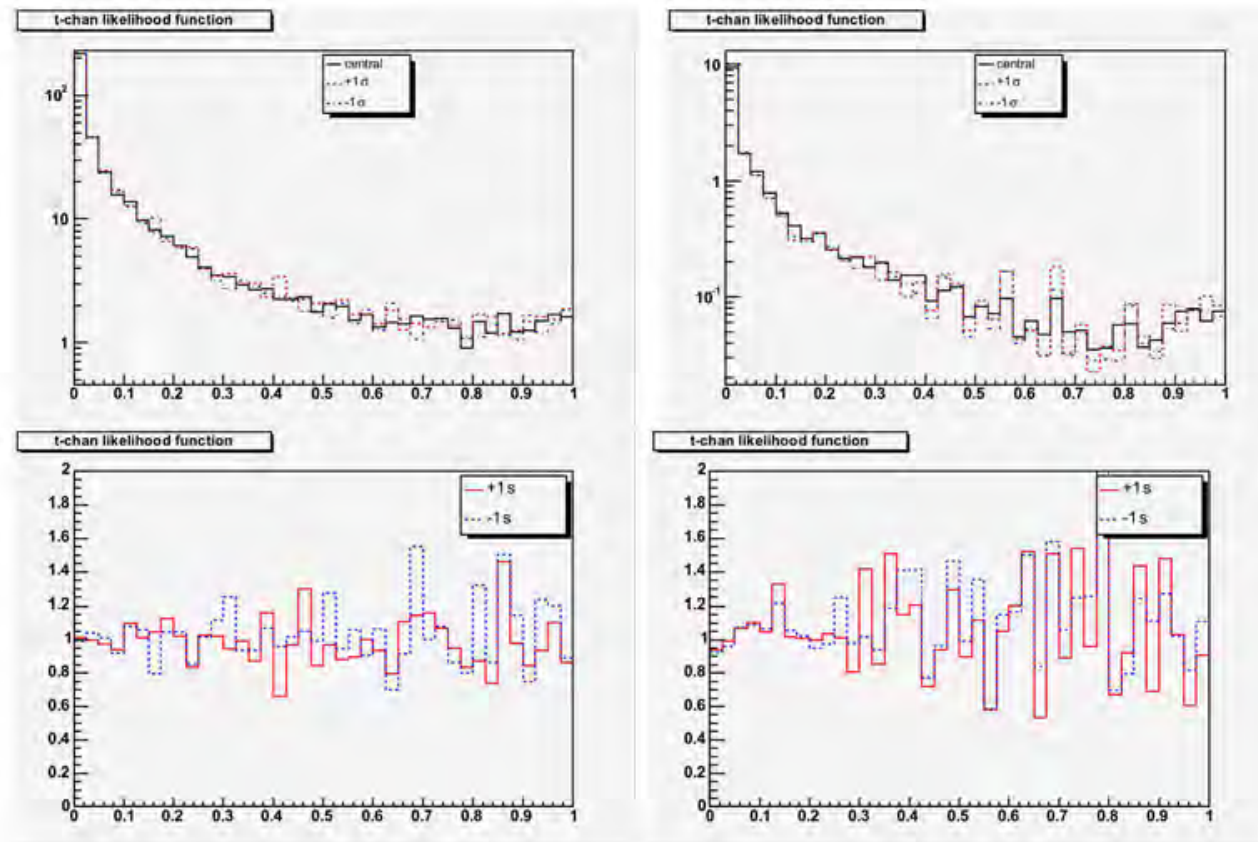

Figure C.4. JES Shape Systematic Uncertainty(WC/Z/ets); compared plot(up) and ratio plot(down) between central and systematic sample 

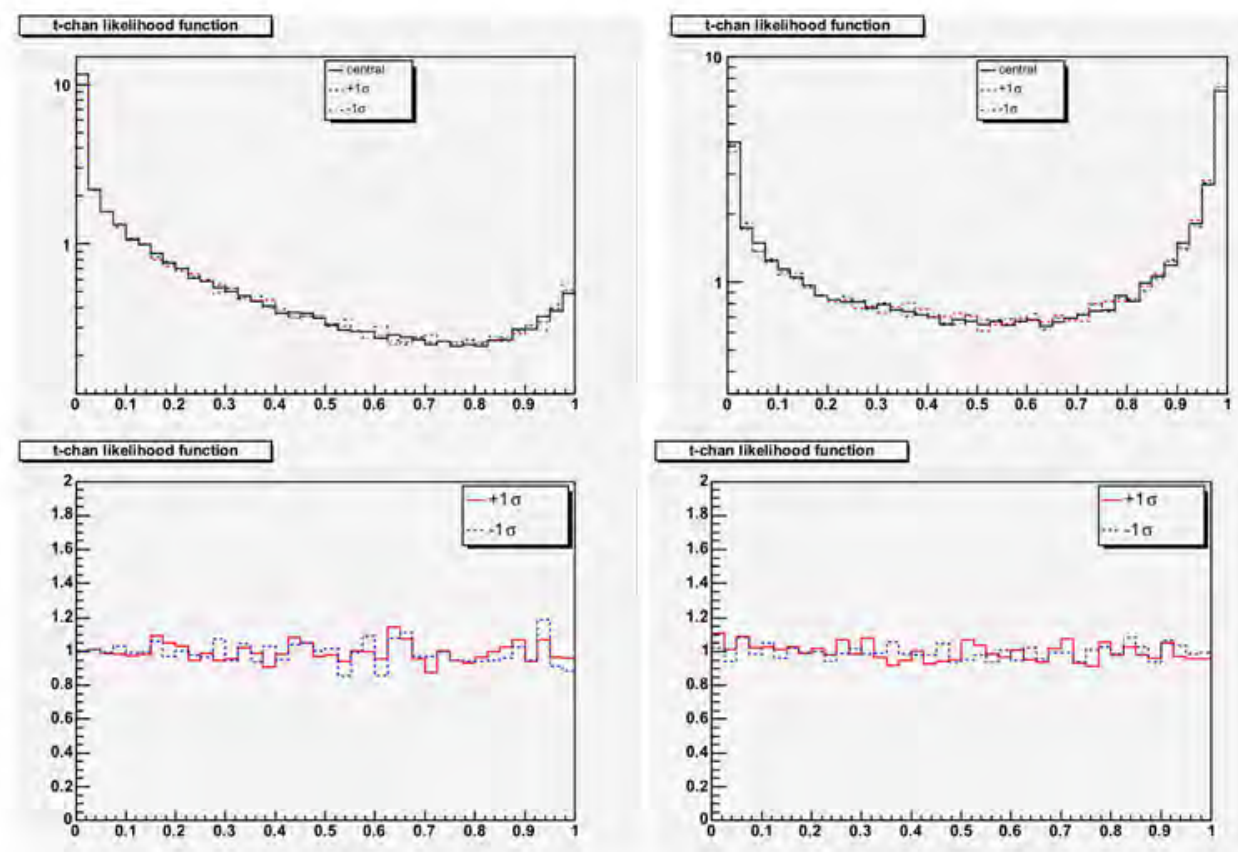

Figure C.5. ISR Shape Systematic Uncertainty(s-/t-channel); compared plot(up) and ratio plot(down) between central and systematic sample 

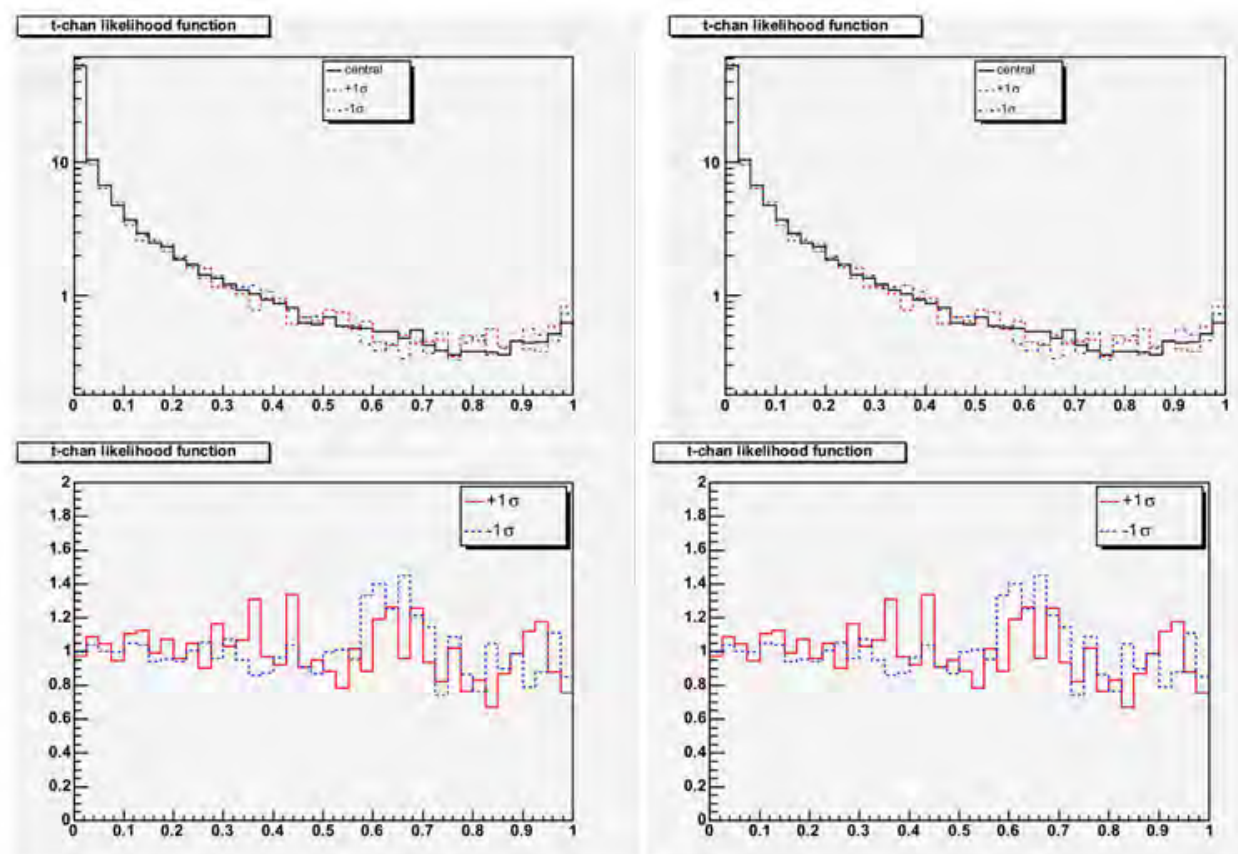

Figure C.6. ISR Shape Systematic Uncertainty(ttbar); compared plot(up) and ratio plot(down) between central and systematic sample 

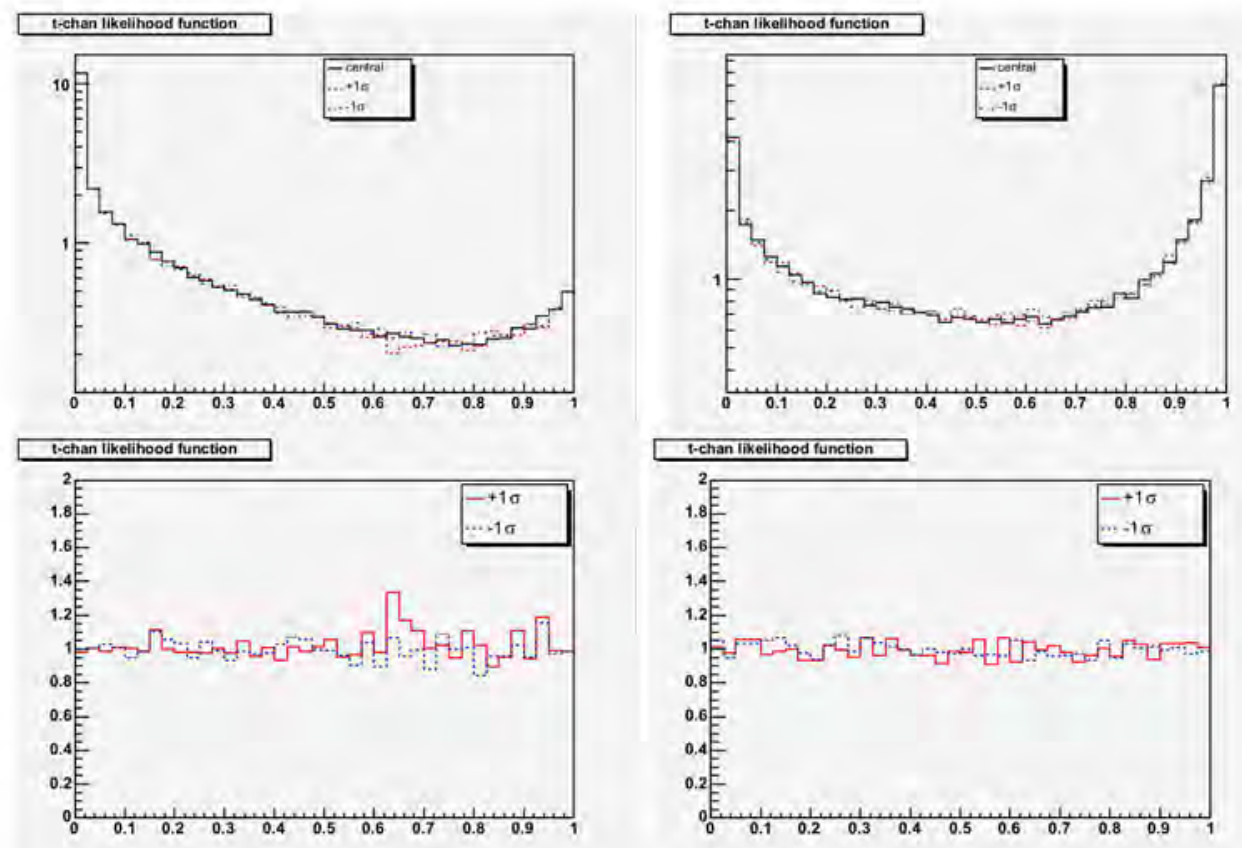

Figure C.7. FSR Shape Systematic Uncertainty(s-/t-channel); compared plot(up) and ratio plot(down) between central and systematic sample

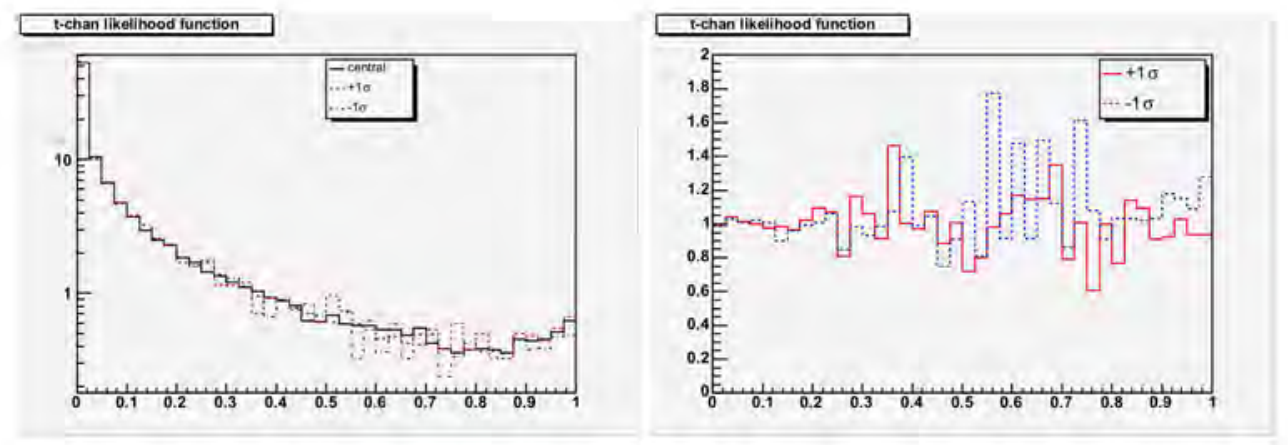

Figure C.8. FSR Shape Systematic Uncertainty(ttbar); compared plot(up) and ratio plot(down) between central and systematic sample 

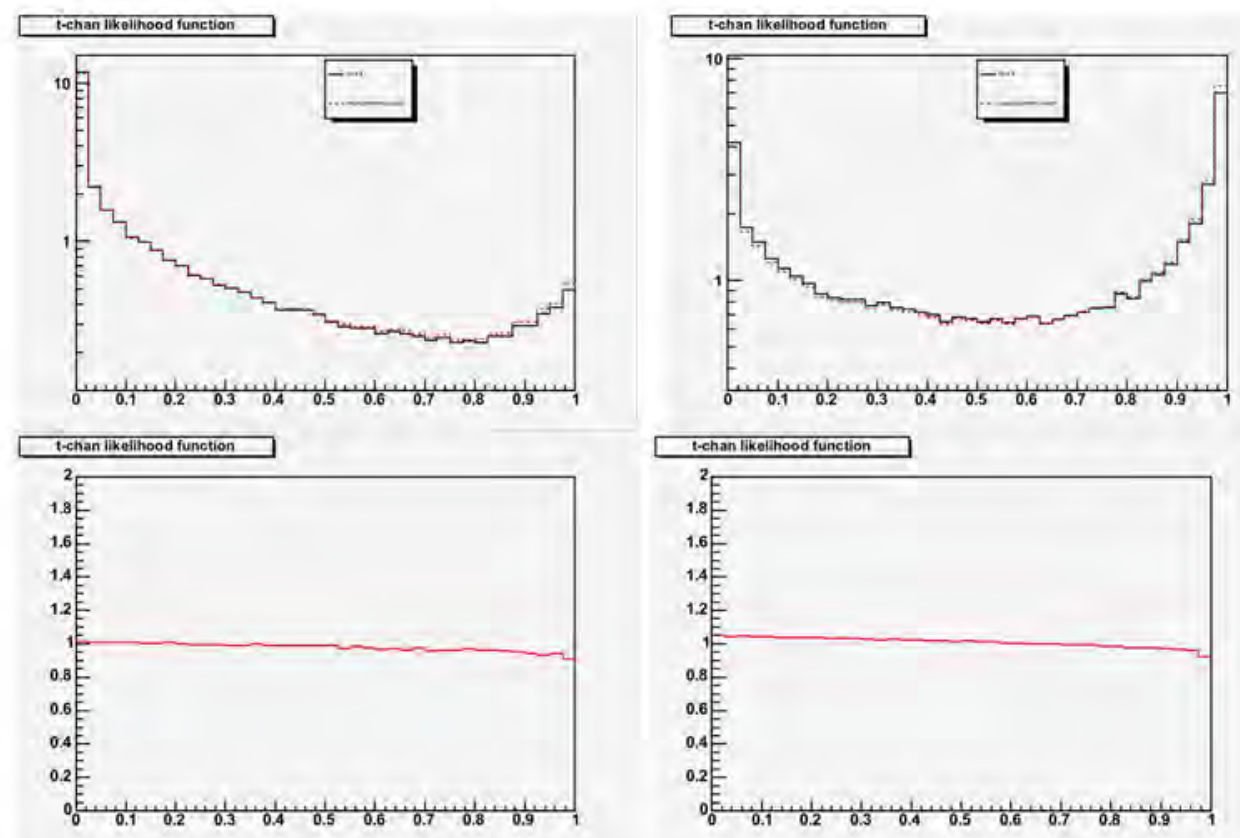

Figure C.9. Eta|2 Shape Systematic Uncertainty(s-/t-channel); compared plot(up) and ratio plot(down) between central and systematic sample
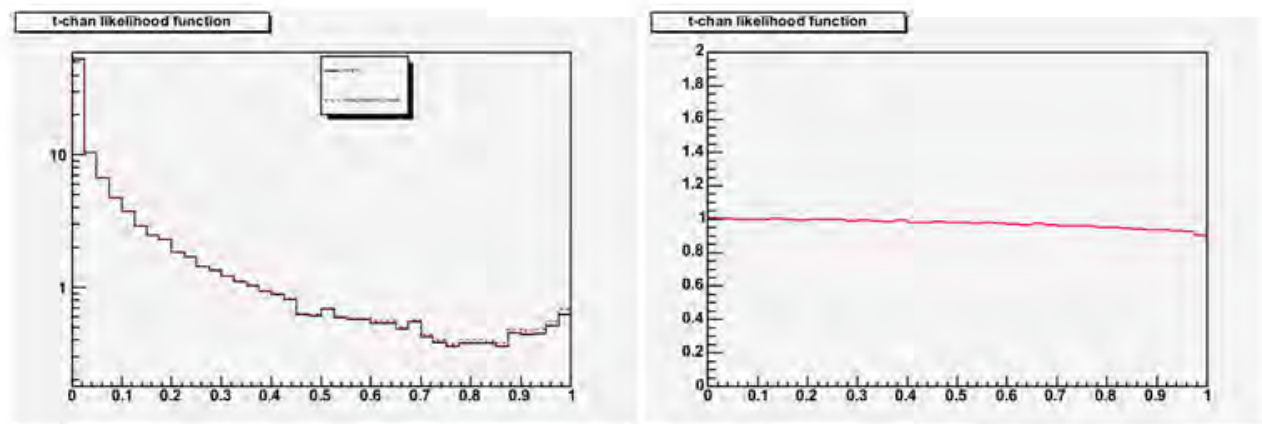

Figure C.10. Eta|2 Shape Systematic Uncertainty(ttbar); compared plot(up) and ratio plot(down) between central and systematic sample 

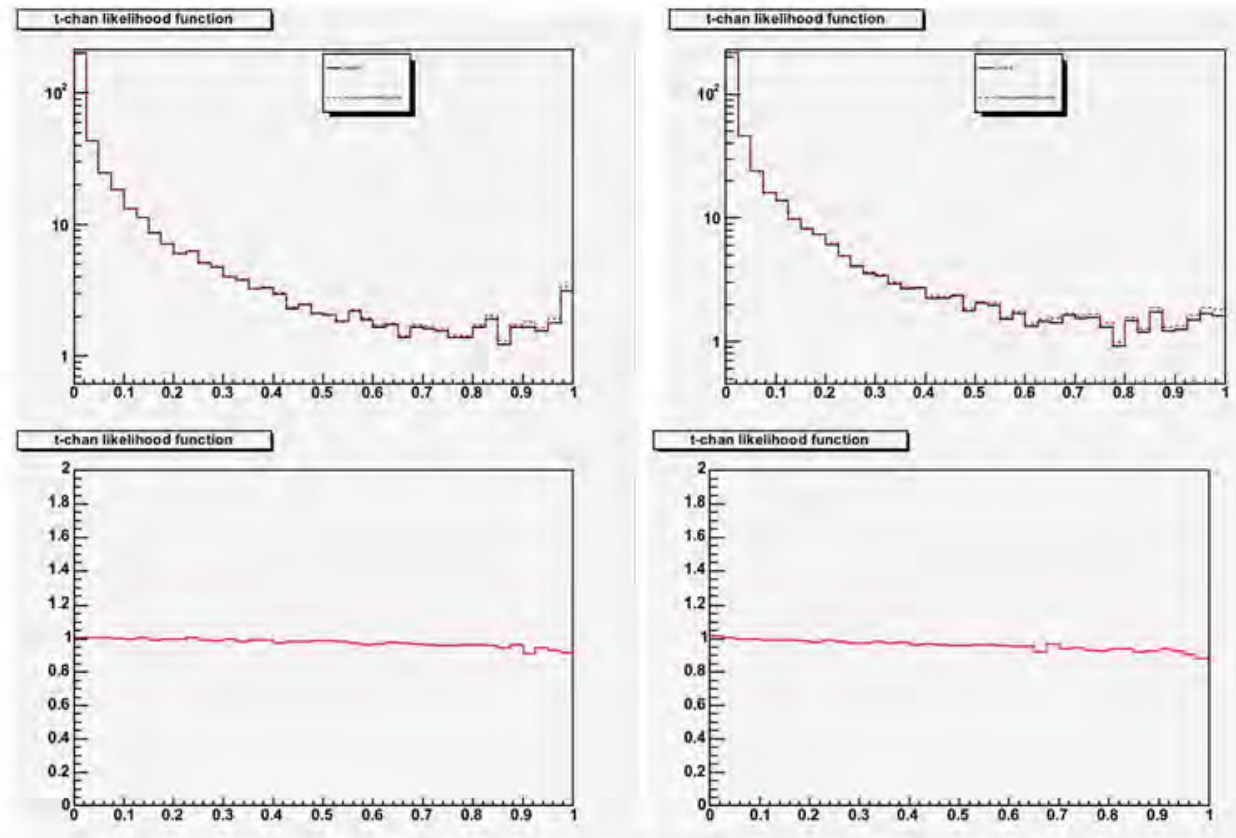

Figure C.11. Eta|2 Shape Systematic Uncertainty(Wbb/WC); compared plot(up) and ratio plot(down) between central and systematic sample 

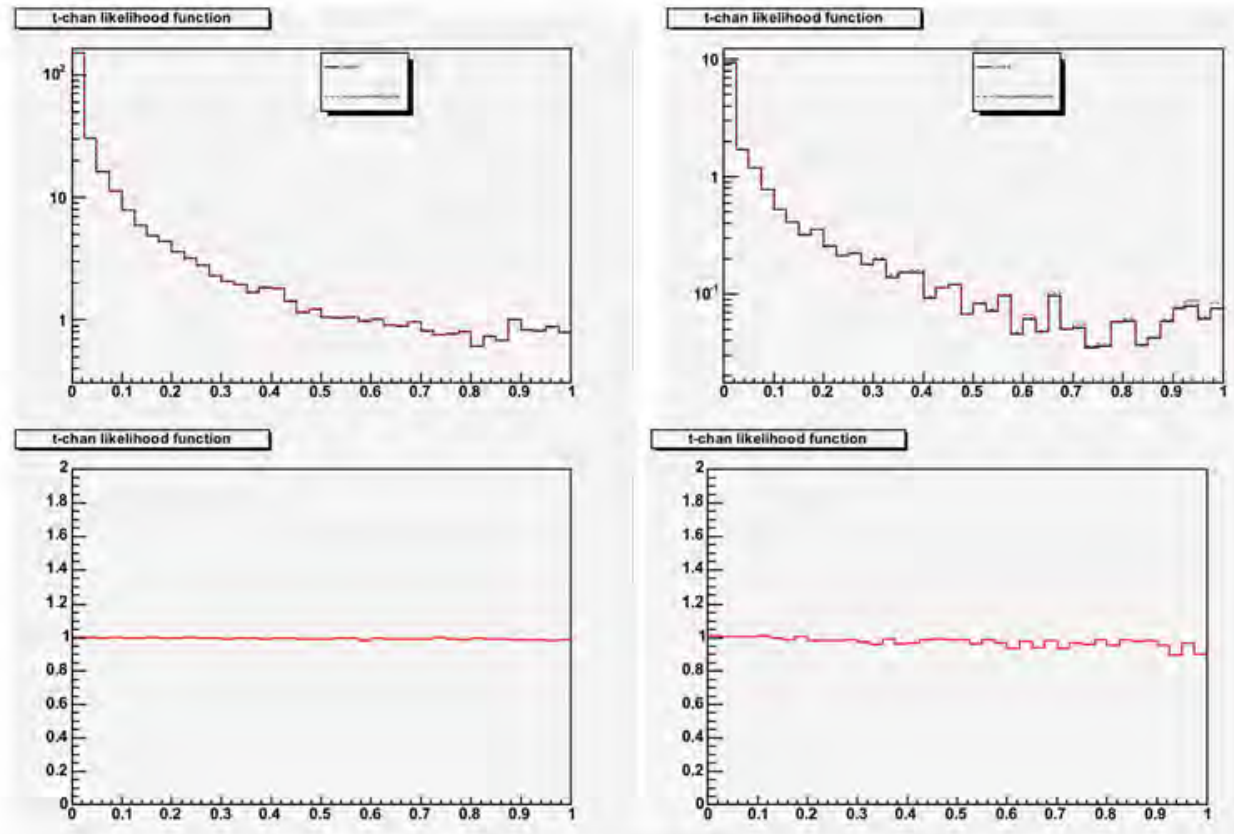

Figure C.12. Eta|2 Shape Systematic Uncertainty(mistag/Z $/$ ets); compared plot(up) and ratio plot(down) between central and systematic sample 

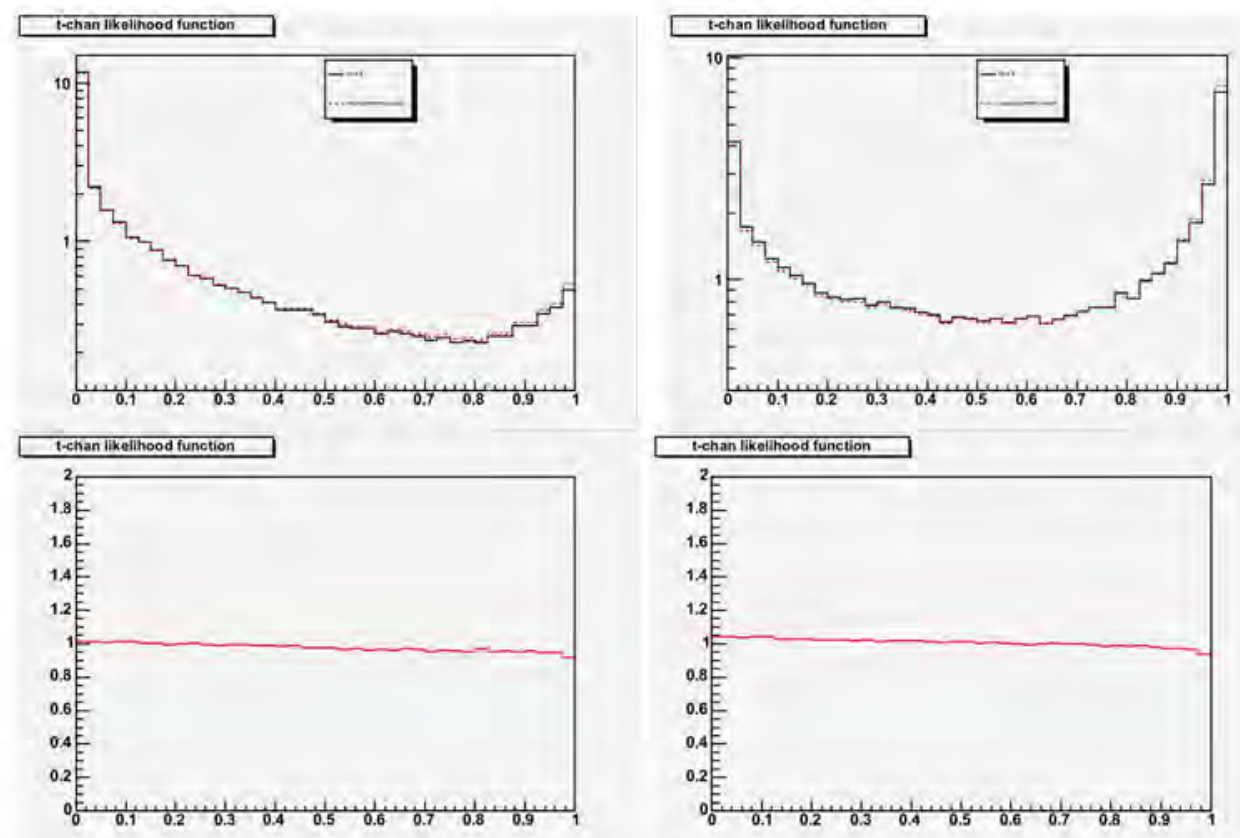

Figure C.13. DeltaR|2 Shape Systematic Uncertainty(s-/t-channel); compared plot(up) and ratio plot(down) between central and systematic sample
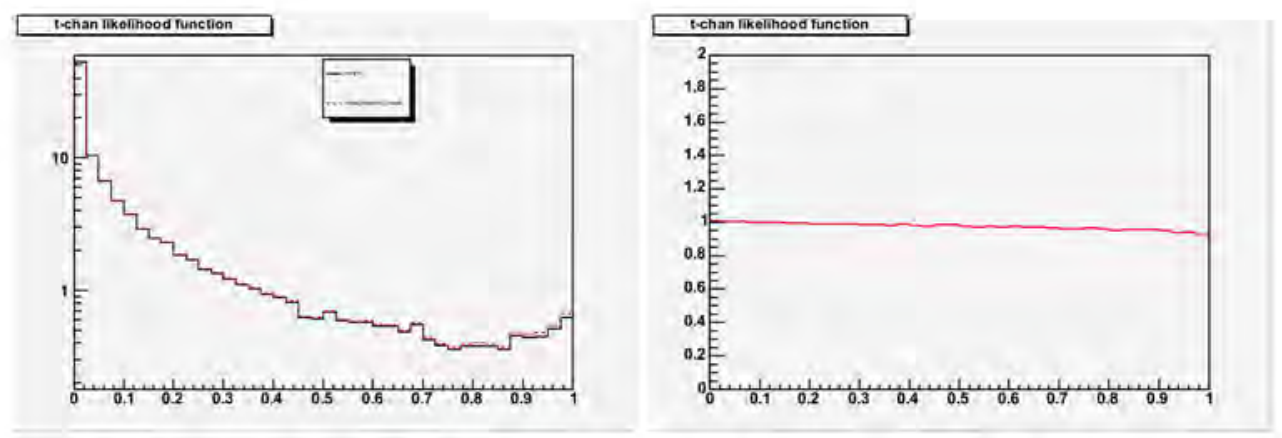

Figure C.14. DeltaR|2 Shape Systematic Uncertainty(ttbar); compared plot(up) and ratio plot(down) between central and systematic sample 

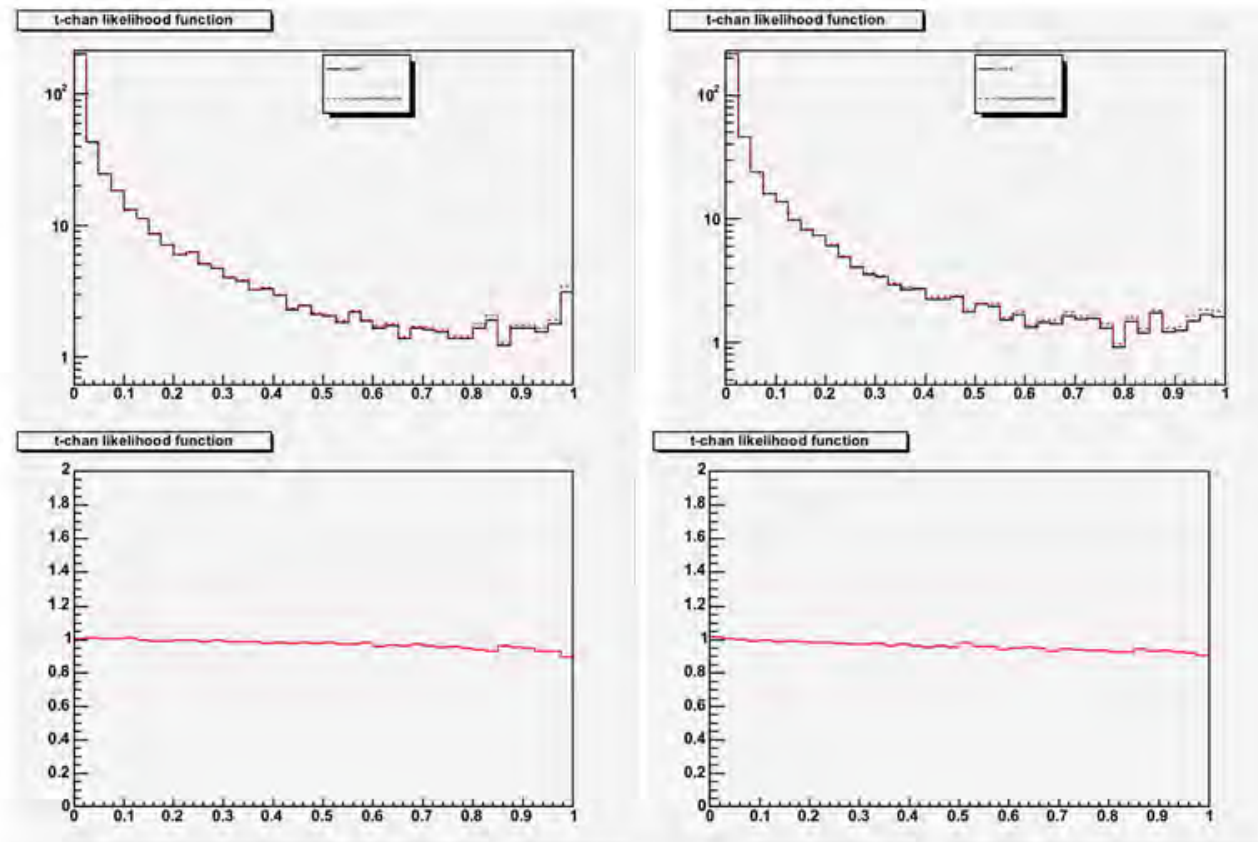

Figure C.15. DeltaR/2 Shape Systematic Uncertainty(Wbb/WC); compared plot(up) and ratio plot(down) between central and systematic sample 

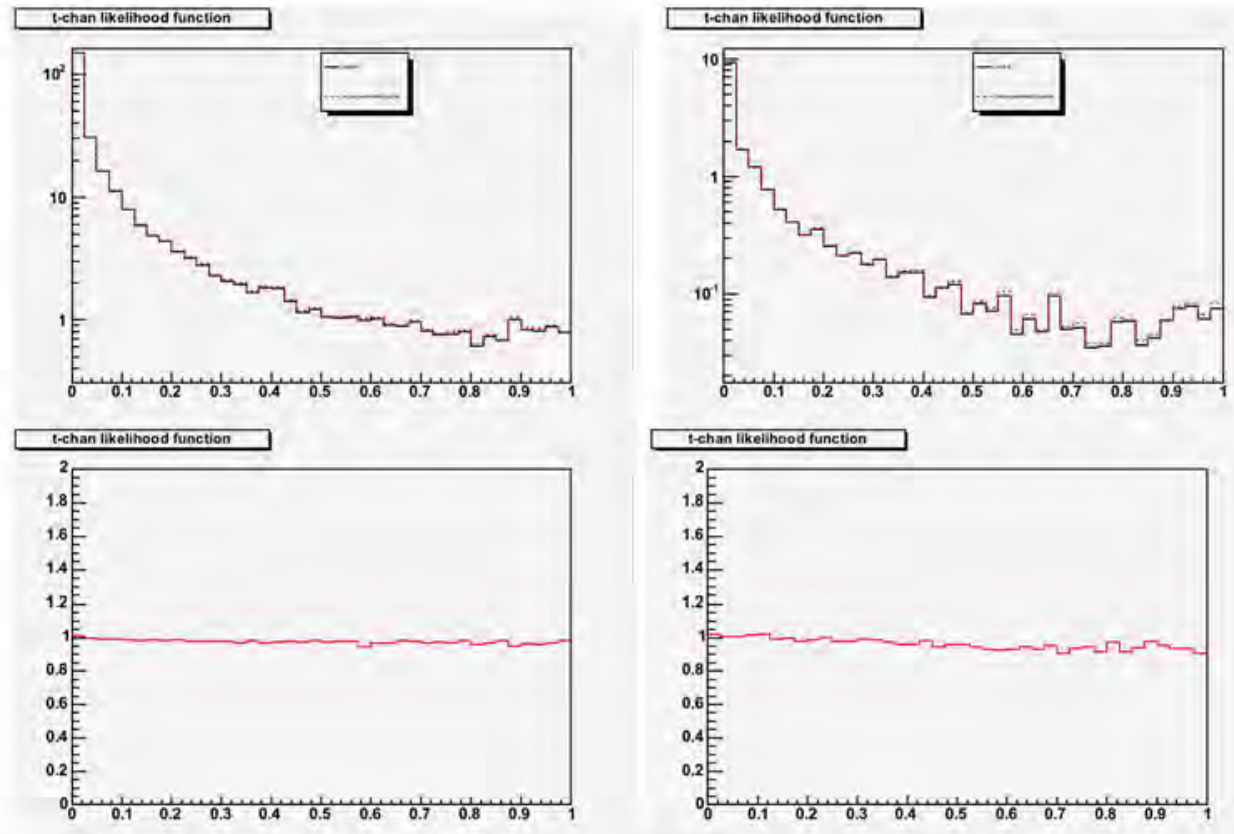

Figure C.16. DeltaR|2 Shape Systematic Uncertainty(mistag/Z|ets); compared plot(up) and ratio plot(down) between central and systematic sample 

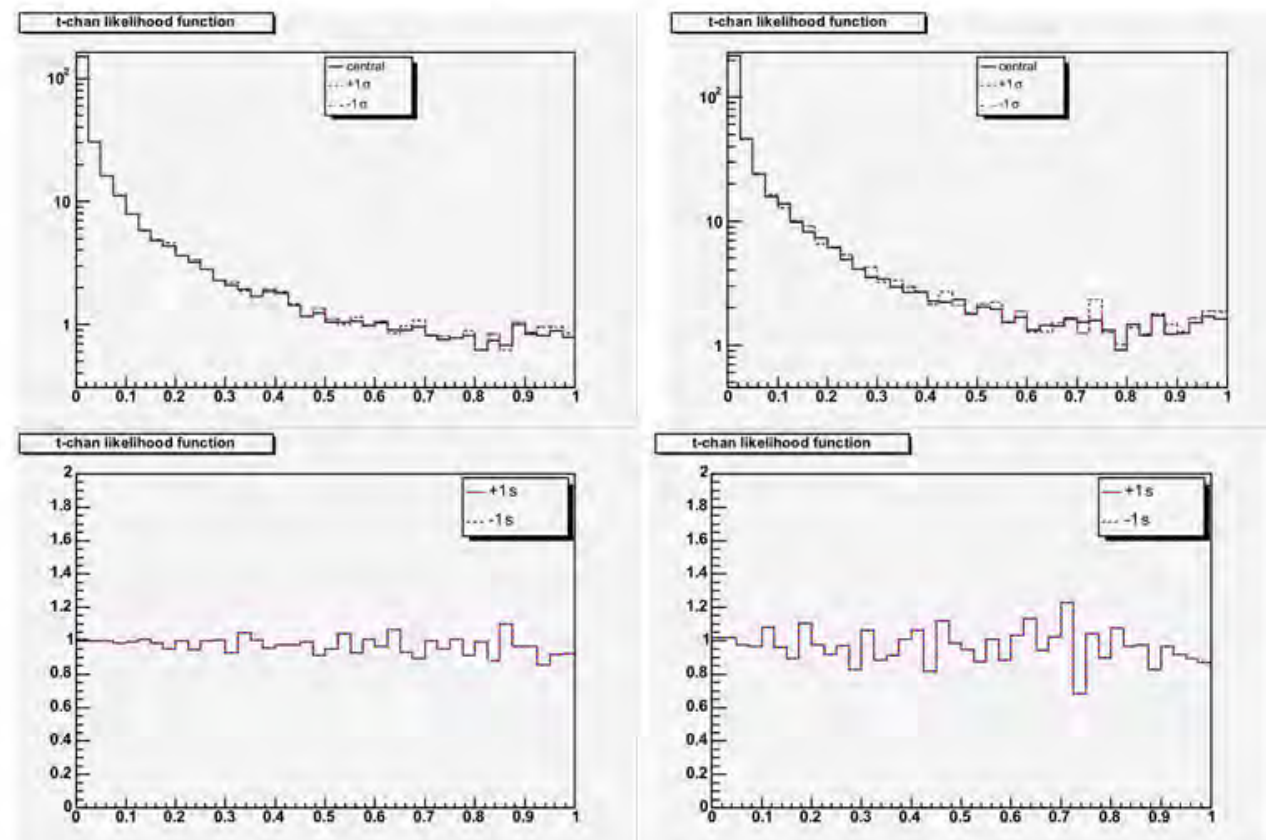

Figure C.17. NNbtag Shape Systematic Uncertainty(Diboson/WC); compared plot(up) and ratio plot(down) between central and systematic sample
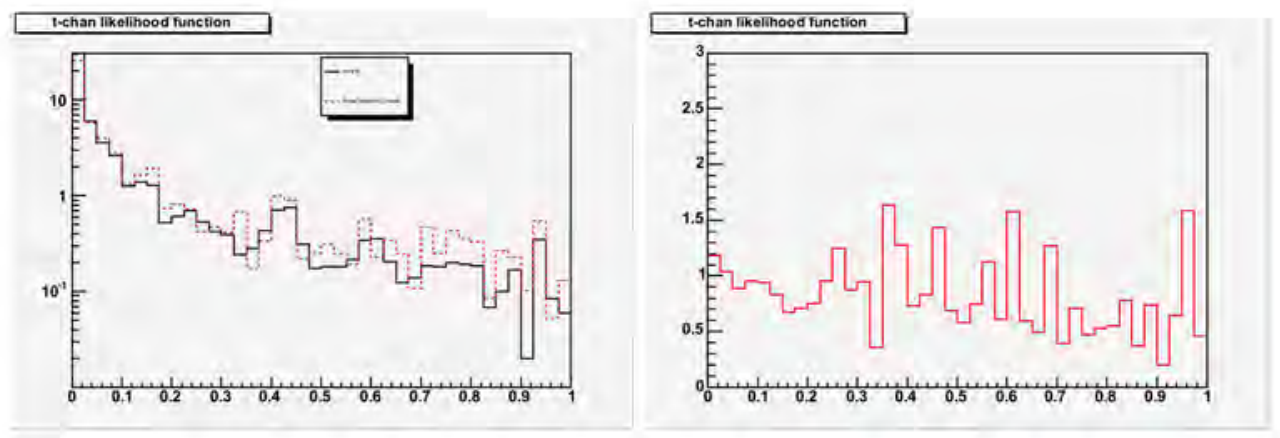

Figure C.18. Nonwflav Shape Systematic Uncertainty(nonw); compared plot(up) and ratio plot(down) between central and systematic sample 


\section{Bibliography}

11] D. Acosta et al. [CDF Collaboration], Phys. Rev. Lett. 95, 102002 (2005) [hep-ph/0505091]

[2] V. M. Abazov et al. [D0 Collaboration], arXiv.0801.1326

[3] J. Swain and L. Taylor, Phys. Rev. D58, 093006 (1998) [hep-ph/9712420]

[4] N. Kidonakis "Single top quark production at the Fermilab Tevatron.Threshold resummation and finite-order soft gluon corrections" Phys. Rev, D 74, 114012(2006)

[5] K. Goulianos Diffractive and Total Cross Section at Tevatron and LHC

[6] T. Aaltonenet al.[The CDF collaboration], "First Observation of Electroweak Single Top quark Production" arXiv.0903.0885[hep-ex].

[7] V. M. Abazovet al.[The Do Collaboration], "Observation of Single Top Quark Production" arXiv.0903.0850[hep-ex].

[8] T. Aaltonenet al.[CDF Collaboration], "Measurement of the Single Top Quark Production Cross Section at CDF" Phys. Rev. Lett 101, 252001 (2008) arXiv.0809.2581[hep-ex]

[9] V. M. Abazovet al.[The Do Collaboration], "Evidence for production of single top quarks and first direct measurement of $\mid V($ tb) $\mid "$ Phys. Rev. Lett $98,181802(2007)$

[10] V. M. Abazovet al.[The D0 Collaboration], "Evidence for production of single top quarks" Phys. Rev. D 78, 012005 (2008)

[11] G. Mahlon, S. Parke "Improved Spin Basis for Angular Correlation Studies in Single Top Quark Production at the Tevatron" arXiv.9611367[hep-ex]

[12] G. Mahlon "Spin Polarization in Single Top Events" arXiv.9811219[hep-ex] 
[13] V. M. Abazovet al.[The D0 Collaboration] "Search for Anomalous Wtb Coupling in Single Top Quark Production" Phys. Rev. Lett 101, 221801 (2008)

[14] T. Stelzer, Z. Sullivan and S. Willenbrock, Phys. Rev. D 58, 094021 (1998) [arXiv.hep-ph/9807340].

[15] G. Abbiendi et al. [OPAL Collaboration], Phys. Lett. B 444, 539 (1998) [arXiv.hep-ex/9808006].

[16] T. Aaltonen et al. [CDF Collaboration], arXiv.0812.3400 [hep-ex].

[17] T. M. P. Tait and C. P. P. Yuan, Phys. Rev. D 63, 014018 (2001) [arXiv.hep$\mathrm{ph} / 0007298]$.

[18] S.R. Budd, C.I. Ciobanu, J-E. Jung, T.R. Junk, S-B. Kim, "Measurement of Single Top Production using Likelihood Functions with $3.2 \mathrm{fb}^{-1}$ of Data", CDF Note 9695 (2009).

[19] Florencia Canelli, Bruno Casal Laraña, Peter Dong, Bernd Stelzer, Rainer Wallny "Measurement of Single Top Quark Production in 3.2/fb of CDF Data Using a Matrix Element Technique", CDF Note 9703 (2009).

[20] D. Hirschbuehl, J. Lueck, Th. Muller, A. Papaikonomou, W. Wagner, "Search for single top-quark production with neural networks using 3.2 $\mathrm{fb}^{-1 "}, \mathrm{CDF}$ Note $9684(2009)$.

[21] B. Alvarez et al., "Search for Single Top Quark Production using Boosted Decision Trees in $3.2 \mathrm{fb}^{-1}$ of CDF Data", CDF Note 9686 (2009).

[22] A. Apresyan,D. Bortoletto, F. Margaroli, K. Potamianos"Measurement of the single top production cross section in the met plus lets sample", CDF Note 9589 (2009).

[23] K. Nakamura and S. Kim, "Single Top Quark s-channel Search Update with $3.2 \mathrm{fb}^{-1}$ data", CDF Note 9702 (2009).

[24] B. Alvarez et al., Combination of the Single-Top Searches with $3.2 \mathrm{fb}^{-1}$, CDF Note 9706 .

[25] B. W. Harris, E. Laenen, L. Phaf, Z. Sullivan and S. Weinzierl, Phys. Rev, D 66, 054024 (2002) [arXiv.hep-ph/0207055].

[26] N. Kidonakis, Phys. Rex. D 74, 114012 (2006) [arXiv.hep-ph/0609287]. 
[27] H. Stadie, W. Wagner, H. Wenzel, and Th. Muller, "Vxprim in Run II", CDF Note 6047

[28] T. Junk, "Sensitivity, Exclusion and Discovery with Small Signals, Large Backgrounds, and Large Systematics", CDF Note 8128.

[29] MadGraph/MadEvent v4. The New Web Generation Johan Alwall, et al, JHEP 0709.028,2007, arXiv.0706.2334.

[30] W helicity in top decay results are summarized at http://www-cdf.fnal.gov/physics/new/top/public_tprop.html

[31] J. Lück, Diplom thesis, University of Karlsruhe, FERMILAB- MASTERS2006-01 (2006).

[32] H. L. Lai, J. Huston, S. Kuhlmann, J. Morfin, F, Olness. J.F.Owens, J. Pumplin, W. K. Tung "Global QC Analysis of Parton Structure of the Nucleon. CTEQ5 Parton Distributions" [arXiv.hep-ph/9903282]

[33] D. Hare, E. Halkiadakis, T. Spreitzer, "Electron ID Efficiency and Scale Factor for Winter 2007 Analuses", CDF Note 8614.

[34] F. Garberson, J.R. Incandela, C. Neu,"SecVtx B-Tag Efficiency Measurement Using Muon Transverse Momentum for $1.2 \mathrm{f} \mathrm{b}^{-1}$ Analyses", CDF Note 8640 .

[35] Franklin, Grinstein, Guimarães da Costa, Lamon, Schwarz, Taffard, Zaw, "Heavy-Flavor Content of the W+Jets Sample", CDF Note 8765.

[36] Franklin, Grinstein, Guimarães da Costa, Sherman, Zaw,"Calibration of Heavy-Flavor Production in QCD Data", CDF Note 8768.

[37] Dante Amidei, Clark Cully, Peter Dong, Sebastian Grinstein, Veronica Sorin, Bernd Stelzer, Rainer Wallny, "Calibration of Heavy-Flavor Production in W+1 Jet Data", CDF Note 9187

[38] T. Aaltonen,et al., "First Measurement of W Boson Production in Association with a Single Charm Quark in pp Collisions at ${ }^{\mathrm{p}} \overline{\mathrm{s}} / 1.96 \mathrm{TeV}$ ", Phys. Rev. Lett. 100, 091803 (2008)

[39] Young-Kee Kim, Un-Ki Yang,"Unitial state gluon radiation studies on Drell-Yan data for top-pair production in hadron collider", CDF Note 6804

[40] T. S|ostrand,et al., "High-Energy-Physics Event Generation with PYTHIA 6.1" (2001) [arXiv.hep-ph/0010017] 
[41] Franklin, Grinstein, Guimarães da Costa, Sherman, Zaw. "Calibration of Heavy-Flavor Production in QCD Data", CDF Note 8768

[42] Sarah Budd, Top Junk, "SECVTX Mistag Parameterization for Winter 2006", CDF Note 8072

[43] Thomas Junk, "Frequentist Exclusion and Discovery of Signals in Combined Searches with Large Systematic Uncertianties on the Background" CDF Note 6525

[44] Thomas Junk, "Sensitibity, Exclusion and Discovery with Small Signals, Large Backgrounds, and Large Systematic Uncertainties" CDF Note 8128

[45] C. Blocker, J. Conway, L. Demortier, J. Heinrich, T, Junk, L. Lyons, G. Punzi "Simple Facts about P-Value"

[46] J. Heinrich, C. Blocker, J. Conway, L. Demortier, L. Lyons, G. Punzi, P. Sinervo. "Interval estimation in the presence of nuisance parameters. 1. Bayesian approach" CDF Note 7117

[47] Joel Heinrich, "Bayesian limit software. multi-channel with correlated backgrounds and efficiencies" CDF Note 7587

[48] J. Adelman et al. "Determination of the Jet Energy Scale at the Collider Detector at Fermilab" [arXiv.hep-ph/0510047]

[49] D. Acosta et al. "A First Look at the CLC Luminosity Measurements" CDF Note 6052

[50] S. Jindariani et al. "Luminosity Uncertainty for Run 2 up until August 2004" CDF Note 7446

[51] Nate Goldschmidt, Ken Bloom, David Gerdes, Dan Amidei. "Measurement of the Helicity of W Bosons Produced in Top-Quark Decay" CDF Note 6617

[52] Christopher Neu, The CDF Collaboration, "CDF b-tagging. Efficiency and Mistag Measurement" CDF Note 8102

[53] Peter Dong, Berud Stelzer, "Reduction of QCD background in lepton + lets events" CDF Note 8295

[54] J. Charles et al. "CP Violation and the CKM Matrix.Assessing the Impact of the Asymmetric B Factoreis" [arXiv.hep-ph/0406184]

[55] A. Hocker, H. Lacker, S. Laplace, F. Le Diberder, "A New Approach to a Global Fit of the CKM Matrix" [arXiv.hep-ph/0104062] 